(c) 2008 Sarah Rebecca Budd 


\title{
SEARCH FOR SINGLE TOP QUARK PRODUCTION WITH THE CDF RUN II DETECTOR USING A MULTIVARIATE LIKELIHOOD METHOD
}

\author{
BY \\ SARAH REBECCA BUDD \\ B.A., Lewis and Clark College, 2003 \\ M.S., University of Illinois at Urbana-Champaign, 2004

\section{DISSERTATION} \\ Submitted in partial fulfillment of the requirements \\ for the degree of Doctor of Philosophy in Physics \\ in the Graduate College of the \\ University of Illinois at Urbana-Champaign, 2008
}

\author{
Urbana, Illinois
}

Doctoral Committee:

Associate Professor Kevin Pitts, Chair

Professor Tony Liss, Director of Research

Professor Michael Stone

Professor Scott Willenbrock 


\section{Abstract}

The top quark is most often produced in $t \bar{t}$ pairs via the strong interaction, however, electroweak production of a single top quark is also possible. Electroweak or 'single-top' quark production is more difficult to observe than $t \bar{t}$ production. A measurement of single-top quark production can provide an interesting test of the Standard Model as well as a measurement of the CKM matrix element $V_{t b}$.

This thesis describes a measurement of single-top quark production in $p \bar{p}$ collisions at $\sqrt{s}=1.96 \mathrm{TeV}$ using the CDF II detector at the Fermilab Tevatron. This search is performed using a Multivariate Likelihood technique with $2.2 \mathrm{fb}^{-1}$ of data. The observed cross section for the combined $t$-channel and $s$-channel single top cross sections $\left(\sigma_{t}\right.$ and $\sigma_{s}$ ) is calculated to be $\sigma_{s}+\sigma_{t}=1.7_{-0.8}^{+0.9}$. A measurement of $V_{t b}$ is derived from this cross section, with the value of $\left|V_{t b}\right|=$ $0.77_{-0.21}^{+0.18}($ exp. $) \pm 0.07$ (theor.), where here the experimental and theoretical errors come from the errors associated with the observed and theoretical single-top cross sections respectively. The probability that this result originates from a background fluctuation, is measured to be $2.40 \times 10^{-2}$ corresponding to $2.0 \sigma$ with an expected probability of $2.32 \times 10^{-4}$ corresponding to $3.50 \sigma$. 
To my sister, MaryBeth, my best friend forever.

Readers looking for the next Great American Novel are humbly suggested that they are perusing the dissertation of the wrong Ms. Budd. 


\section{Acknowledgments}

I would like to thank the people who have been my official advisers, Tony Liss and Tom Junk. I would also like to thank Kevin Pitts, who stepped in to help me prepare for my prelim exam. Thanks to the two University of Mlinois postdoctoral researchers who helped guide my studies and service work: Catalin Ciobanu who mentored me during my first several years and Xiaojian Zhang who I had the pleasure to accompany on many trips into the collision hall. Also thanks to the poctdocs Heather Gerberich, Olga Norniella, Anyes Taffard and Gregory Veramendi, the HEPG staff including Donna Guzy, Rene Dunham, Tonya Ayers, and Dave Lesny, and my fellow University of Illinois CDF graduate students Alice Bridgeman, Ulysses Grundler, James Kraus, Christopher Marino, Ed Rogers, Greg Thompson, and Ben Carls.

Thanks to all the members of the CDF single-top group, especially the UCLA group (Florencia Canelli, Peter Dong, Bernd Stelzer, and Rainer Wallny), and the Karlsruhe group (Dominic Hirschbühl, Jan Lück, Thomas Müller, Adonis Papaikonomou, Thomas Peiffer, Manuel Renz, Svenja Richter, Irja Schall, Wolfgang Wagner, and Jeannine Wagner-Kuhr). Wolfgang Wagner in particular helped me integrate into the group when I first arrived at Fermilab, and Bernd Steltzer was always there to answer my questions. I also acknowledge all other single-top group members (Craig Group, Kevin Lannon, Charles Plager, Bruno Casal Laraña, Alberto Ruiz, Rocio Vilar, Koji Nakamura, Shinhong Kim and Ji-eun Jung). Charles Plager notably provided many code tools used in this analysis.

I would like to acknowledge the other CDF collaborators, especially the CDF and CDF Top Group leadership including Robin Erbacher, Florencia Canelli, Kirsten Tollefson, Robert Roser, and Jacobo Konigsberg. Also thanks to internal analysis reviewers Mel Shochet, Ulrich Husemann, Robert Roser, Takasumi Maruyama and David Stuart. I would finally like to acknowledge members of the b-tagging and muon detector groups. Also thanks to Jon Stone who helped with the editing.

Finally, thanks to my parents Tim and Elizabeth Budd, my sister MaryBeth Budd, my boyfriend James Kraus and other family, friends and mentors too plentiful to list. The $\mathrm{PhD}$ process has not always been an easy one for me, and I appreciate all your love and support more than I can say.

This thesis was supported in part by the United States Department of Energy under grant DE-FG02-91ER40677. 


\section{Table of Contents}

List of Tables $\ldots \ldots \ldots \ldots \ldots$. . . . . . . . . . . . . . . .

List of Figures .................... ix

1 Introduction ..................... 1

2 The Tevatron and the CDF II Detector . . . . . . . . 2

2.1 The Fermilab Accelerator Complex . . . . . . . . . . . . . 2

2.1 .1 Luminosity . . . . . . . . . . . . . . 3

2.2 The CDF Detector . . . . . . . . . . . . . . 4

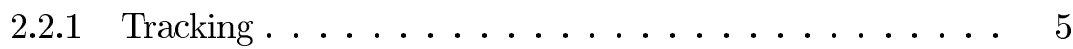

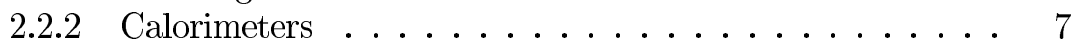

2.2.3 Muon Chambers . . . . . . . . . . . . . . . . . 7

2.3 Trigger System . . . . . . . . . . . . . . . . . 9

3 The Standard Model and the Top Quark . . . . . . . . . . 11

3.1 The Standard Model of Particle Physics . . . . . . . . . . . . . 11

3.1.1 The Weak Force and CKM Mixing . . . . . . . . . . . 12

3.1.2 Parton Distribution Functions . . . . . . . . . . . 13

3.2 The Top Quark . . . . . . . . . . . . . . . . . . . . . . 15

3.2.1 Top Quark Production via the Strong Interaction . . . . . 15

3.3 Single-Top Quark Production . . . . . . . . . . . . . . . 17

3.3.1 Single-Top Quark Polarization . . . . . . . . . . 18

3.3.2 $V_{t b}$ and Single-Top Production . . . . . . . . . . 20

3.3.3 Single-top as a Test of New Physics . . . . . . . . . . 20

3.3.4 Beyond Single-Top . . . . . . . . . . . . . . . . . . 22

4 Event Selection and Particle Reconstruction _....... 23

4.1 Triggers . . . . . . . . . . . . . . . . 23

4.1.1 Central Electron Triggers $\ldots \ldots \ldots \ldots . \ldots . \ldots 23$

4.1 .2 Plug Electron Triggers . . . . . . . . . . . . . . . . . . 24

4.1.3 Central Muon Triggers . . . . . . . . . . . . . . . . 24

4.1 .4 CMX Muon Triggers . . . . . . . . . . . . . . . . 24

4.1.5 Data Samples . . . . . . . . . . . . . . . . . 25

4.2 Event Reconstruction . . . . . . . . . . . . . . . 25

4.2.1 Central (CEM) Electrons . . . . . . . . . . . . 25

4.2 .2 Plug Electron Selection _. . . . . . . . . . . . 26

4.2 .3 Muon Selection . . . . . . . . . . . . . . . . . . 29

4.2 .4 Jet Selection . . . . . . . . . . . . . . . . . . 31

4.2.5 Jet Energy Corrections . . . . . . . . . . . . . . . . 31

4.2 .6 B Jet Identification . . . . . . . . . . . . . . . . . . . . . . . . . . . . . .

4.2.7 Neutrino Reconstruction . . . . . . . . . . . . . . 34

4.3 Event Selection Cuts . . . . . . . . . . . . . . . . 35

4.3 .1 Background Vetoes . . . . . . . . . . . . . 36

4.3 .2 QCD Veto ................ 36 
5 Signal and Background Modeling _ . . . . . . . . . 38

5.1 Monte Carlo Event Generation . . . . . . . . . . . . . . . 38

5.1.1 Parton Shower Generators . . . . . . . . . . . . . 38

5.1 .2 Matrix Element Generators . . . . . . . . . . . . . 39

5.2 Detector Simulation . . . . . . . . . . . . . . . . 40

5.3 Single Top Modeling . . . . . . . . . . . . . . . . . . . . . 40

5.3.1 Single Top Monte Carlo . . . . . . . . . . . . . . . . . 40

5.3 .2 Event Detection Efficiencies . . . . . . . . . . . . . 41

5.4 Background Modeling . . . . . . . . . . . . . . . . . 44

5.4.1 The Mistag Matrix and Mistag Asymmetry Corrections . 44

$5.4 .2 \quad \mathrm{~W}+$ Jets and $\mathrm{Z}+$ jets $\ldots \ldots \ldots \ldots \ldots \ldots$

$5.4 .3 t \bar{t}$ and Diboson . . . . . . . . . . . . . . 49

5.4 .4 QCD . . . . . . . . . . . . . . . 49

5.5 Total Signal and Background Predictions . . . . . . . . . 50

5.5 .1 Background Prediction . . . . . . . . . . . . . 50

5.5.2 Total Signal and Background Estimate . . . . . . . . . . 52

6 Special Techniques . . . . . . . . . . . . . . . 54

6.1 Kinematic Solver and Matrix Element Construction . . . . . . 54

6.1 .1 Kinematic Solver . . . . . . . . . . . . . . . . 54

6.1.2 Matrix Element Calculation . . . . . . . . . . . . . 57

$6.2 \quad B$-Choosing Algorithms . . . . . . . . . . . . . . 59

6.3 Neural Net B Tagger . . . . . . . . . . . . . . . . . . 61

7 Likelihood Function . . . . . . . . . . . . . . 64

7.1 Introduction to Likelihood Functions . . . . . . . . . . . . 64

7.2 The Two-Jet Bin . . . . . . . . . . . . . . . 65

7.3 The Three-Jet Bin . . . . . . . . . . . . . . . 74

7.4 Variable Check Plots . . . . . . . . . . . . . . . . . 83

7.4.1 $\mathcal{L}_{2 t}$ Input Variables . . . . . . . . . . . . . . . . 86

$7.4 .2 \mathcal{L}_{3 t}$ Input Variables . . . . . . . . . . . . . . . . 99

7.4.3 Further Kinematic Modeling Check Plots . . . . . . . . 112

8 Systematics and Statistical Methods _. . . . . . . . 127

8.1 Systematics . . . . . . . . . . . . . . . . . 127

8.2 Statistical Methods . . . . . . . . . . . . . . . . . 132

8.2.1 Likelihood Function . . . . . . . . . . . . . . . 132

8.2 .2 The $p$-value Calculation . . . . . . . . . . . . 133

8.2.3 The Cross Section Measurement . . . . . . . . . . . . . 134

9 Results ...................... 136

9.1 Expected and Observed $p$-value . . . . . . . . . . . 136

9.2 Measurement of the Single-Top Production Cross Section . . . 138

9.2.1 Measurement of $\left|V_{t b}\right| \ldots \ldots \ldots \ldots$

10 Outlook . . . . . . . . . . . . . . . . . . . 140

10.1 CDF Results . . . . . . . . . . . . . . . . . 140

10.2 CDF Combination . . . . . . . . . . . . . . . . 140

10.3 DØ Results . . . . . . . . . . . . . . . . . . . . . . . . 140

10.4 Outlook at CDF . . . . . . . . . . . . . . . 141 
A Mistag Matrix ..................... 145

A.1 Data Samples and Prescription . . . . . . . . . . . . 145

A.2 Method ....................... 145

A.3 Cross Checks . . . . . . . . . . . . . . . . . . 146

A.3.1 Self-Consistency . . . . . . . . . . . . . . . . 146

A.3.2 Predictivity . . . . . . . . . . . . . . . 150

A.4 Mistag Rate Systematics . . . . . . . . . . . . . . . . . . 155

B Shape Systematic Plots . . . . . . . . . . . . . 157

B.1 Shape Systematic Plots, 2-jet Bin . . . . . . . . . . . . . . 157

B.2 Shape Systematic Plots, 3-jet Bin . . . . . . . . . . . . . . . 169

C Muon Chamber Monitoring and Maintenance . . . . . . . 179

C.1 CMP Aging . . . . . . . . . . . . . . . . . . 182

D The CKM Matrix and the Higgs Field . . . . . . . . 185

References ..................... 189

Author's Biography .................... 194 


\section{List of Tables}

3.1 Fermion Properties . . . . . . . . . . . . . . . . . . . . 12

3.2 Boson Properties . . . . . . . . . . . . . . . . . . . . . 12

3.3 Theoretical Single-Top Cross Sections . . . . . . . . . . . 17

4.1 CEM Selection Cuts . . . . . . . . . . . . . . . . 27

4.2 PHX Selection Cuts . . . . . . . . . . . . . . 28

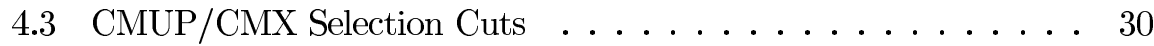

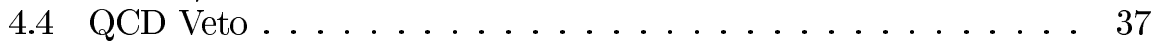

4.5 QCD Efficiencies . . . . . . . . . . . . . 37

5.1 Monte Carlo Samples used in the Single-Top Model . . . . . . . . 40

5.2 Electron and Muon Trigger Efficiencies . . . . . . . . . . . . . 42

5.3 Mistag Asymmetry . . . . . . . . . . . . . . . . . . . 45

5.4 Monte Carlo Samples used in the Z+Jets Model . . . . . . . . 47

5.5 Monte Carlo Samples used in the W+Jets Model . . . . . . . . . 48

5.6 Monte Carlo Samples used for the $t \bar{t}$ and Diboson Samples . . . . 49

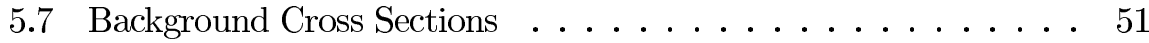

5.8 Dilepton Scale Factor . . . . . . . . . . . . . . . . 51

5.9 Method 2 Background Estimate . . . . . . . . . . . . 52

6.1 Tag Multiplicity . . . . . . . . . . . . . . . . 59

$6.2 \quad B$-Tag Effectiveness . . . . . . . . . . . . . . . 59

$6.3 B$ Choosing Effectiveness of Various Kinematic Variables . . . . 60

$6.4 \quad B$ Choosing Algorithms . . . . . . . . . . . . . . 61

7.1 KS values . . . . . . . . . . . . . . . . . 84

$7.2 \chi^{2} /$ DOF Values . . . . . . . . . . . . . 85

8.1 Systematic Rate Uncertainties for the 2-jet bin, in Percent. . . . 130

8.2 Systematic Rate Uncertainties for the 3-jet bin, in Percent. . . . 131

A.1 Binning used in Tag Matrices. . . . . . . . . . . . . . 146

A.2 Cross Checks for Tight Tag Matrices. . . . . . . . . . . . . . 156

A.3 Summary of Systematic Uncertainties for the Mistag Matrix. . . 156 


\section{List of Figures}

2.1 Schematic overview of the Fermilab accelerator chain. . . . . . 3

2.2 Particle detection at CDF. . . . . . . . . . . . . 4

2.3 The CDF Run II detector . . . . . . . . . . . . . . . 5

2.4 The CDF Run II detector cut away view. . . . . . . . . . . . 6

2.5 Geometrical coverage of the CDF muon chambers, on the $\eta$ - $\phi$ plane. 8

2.6 Diagram of the CDF trigger system. . . . . . . . . . 10

3.1 Parton distribution functions . . . . . . . . . . . . . . . 14

3.2 Top quark production diagrams . . . . . . . . . . . . . 16

$3.3 t$-channel single-top production . . . . . . . . . . . 17

$3.4 s$-channel single-top production . . . . . . . . . . . . 18

3.5 Template histogram of $\cos \left(\theta_{\text {tchan }}\right) \ldots \ldots \ldots \ldots \ldots \ldots$

$3.6 W^{\prime}$ mass limits from $\mathrm{CDF} \ldots \ldots \ldots \ldots \ldots$

4.1 SECVTX tagging diagram $\ldots \ldots \ldots \ldots \ldots \ldots \ldots$

5.1 Single-Top Feynman diagrams . . . . . . . . . . . . . . . 41

5.2 Mistag figure . . . . . . . . . . . . . . 44

5.3 Distribution of the number of jets . . . . . . . . . . 53

$6.1 \Delta P_{b}$ and $\Delta E_{T}$ in the 2-jet bin $\ldots \ldots \ldots \ldots \ldots \ldots$

$6.2 \Delta P_{b}$ and $\Delta E_{T}$ in the 3 -jet bin $\ldots \ldots \ldots \ldots \ldots$

6.3 Raw $M_{\ell \nu b}$ and $M_{\ell \nu b}$ reconstructed with kinematic solver quantities 56

6.4 Log of kinematic solver $\chi^{2}$ template . . . . . . . . . . . . . 57

$6.5 t$-channel matrix element template . . . . . . . . . . 58

6.6 Neural Net B Tagger . . . . . . . . . . . . . . . . . . . . . . 62

6.7 ANN correction functions $\ldots \ldots \ldots \ldots$

7.1 Template histograms of $H_{T}$ and $Q \times \eta$ in the 2-jet bin . . . . 68

7.2 Template histograms of $\cos \theta_{t-\text { chan }}$ and $M_{j 1 j 2}$ in the 2-jet bin . . 69

7.3 Template histograms of $\chi_{t}^{2}$ and $\mathrm{ME}_{t-\text { chan }}$ in the 2-jet bin $\ldots . .70$

7.4 Template histogram of ANN $b$-tag in the 2-jet bin $\ldots \ldots \ldots 71$

7.5 2-jet bin LF template . . . . . . . . . . . . . . . 72

7.6 Stacked histograms of 2-jet likelihood function $\ldots \ldots \ldots . \ldots 73$

7.7 Template histograms of $M_{l v b}$ and $N N(b)$ in the 3 -jet bin . . . . 76

7.8 Template histograms of the number of tagged jets and $Q \times \eta \quad . \quad 77$

7.9 Template histograms of $\Delta R$ and $M_{j j}$ in the 3 -jet bin. . . . . . 78

7.10 Template histograms of $\cos (\theta)_{t c h a n}$ and $p_{T}$ of the $b$ jet in the 3 -jet bin . . . . . . . . . . . . . . . 79

7.11 Template histograms of $P_{T}$ of the lowest momentum jet, and $\eta$ of the reconstructed $W$ boson in the 3 -jet bin . . . . . . . . 80

7.12 Template histograms of the 3 -jet likelihood function $\ldots . . .881$

7.13 Stacked plots of the 3 -jet likelihood function . . . . . . . . . . 82

7.14 Stacked histograms of $H_{T}$ in the tag region $\ldots \ldots \ldots . . . .86$ 
7.15 Stacked histograms of $H_{T}$ in the taggable region $\ldots \ldots \ldots 87$

7.16 Stacked histograms of $Q \times \eta$ in the tag region . . . . . . 88

7.17 Stacked histograms of $Q \times \eta$ in the taggable region $\ldots \ldots \ldots . \quad$. . 89

7.18 Stacked histograms of $\cos \theta_{t-\text { chan }}$ in the tag region $\ldots \ldots \ldots .90$

7.19 Stacked histograms of $\cos \theta_{t-\text { chan }}$ in the taggable region $\ldots . .991$

7.20 Stacked histograms of $M_{j j}$ in the tag region . . . . . . . . 92

7.21 Stacked histograms of $M_{j j}$ in the taggable region . . . . . . . 93

7.22 Stacked histograms of $\log \left(\chi_{t}^{2}\right)$ in the tag region . . . . . . . . 94

7.23 Stacked histograms of $\log \left(\chi_{t}^{2}\right)$ in the taggable region . . . . . . 95

7.24 Stacked histograms of $\log \left(\mathrm{ME}_{t-\text { chan }}\right)$ in the tag region $\ldots . . .96$

7.25 Stacked histograms of $\log \left(\mathrm{ME}_{t-\text { chan }}\right)$ in the taggable region $\ldots 97$

7.26 Stacked histograms of ANN $b$-tag in the tag region . . . . . . 98

7.27 Stacked histograms of $m_{\ell \nu b}$ in the tag region . . . . . . . . 99

7.28 Stacked histograms of $m_{\ell \nu b}$ in the taggable region . . . . . . . 100

7.29 Stacked histograms of the number of tags in the tag region . . . 101

7.30 Stacked histograms of smallest $\Delta R$ of any two jets in the tag region102

7.31 Stacked histograms of smallest $\Delta R$ of any two jets in the taggable region . . . . . . . . . . . . . . . . 103

7.32 Stacked histograms of $M_{j j}$ of the two jets not chosen to be the $b$ from top decay in the tag region . . . . . . . . . . . . . 104

7.33 Stacked histograms of $M_{j j}$ of the two jets not chosen to be the $b$ from top decay in the taggable region . . . . . . . . . . 105

7.34 Stacked histograms of $P_{T}$ of the lowest momentum jet in the tag region . . . . . . . . . . . . . . 106

7.35 Stacked histograms of $P_{T}$ of the lowest momentum jet in the taggable region . . . . . . . . . . . . . . 107

7.36 Stacked histograms of $\eta$ of the reconstructed $W$ boson in the tag region . . . . . . . . . . . . . . . . 108

7.37 Stacked histograms of $\eta$ of the reconstructed $W$ boson in the taggable region . . . . . . . . . . . . . . . . . 109

7.38 Stacked histograms of $p_{T}$ of the jet chosen to be the $b$ from top decay in the tag region . . . . . . . . . . . . . . 110

7.39 Stacked histograms of $p_{T}$ of the jet chosen to be the $b$ from top decay in the taggable region . . . . . . . . . . . . . . 111

7.40 Stacked histograms of $\eta$ of the lowest momentum jet in the tag region . . . . . . . . . . . . . . . . 112

7.41 Stacked histograms of $\eta$ of the lowest momentum jet in the taggable region . . . . . . . . . . . . . . . . . 113

7.42 Stacked histograms of $\eta$ of the leading jet in the tag region . . . 114

7.43 Stacked histograms of $\eta$ of the leading jet in the taggable region 115

7.44 Stacked histograms of $\eta$ of the second jet in the tag region . . . 116

7.45 Stacked histograms of $\eta$ of the second jet in the taggable region . 117

7.46 Stacked histograms of $\eta$ of the third jet in the tag region . . . . 118

7.47 Stacked histograms of $\eta$ of the third jet in the taggable region . . 119

7.48 Stacked histograms of $\phi$ of the lowest momentum jet in the tag region . . . . . . . . . . . . . . . 120

7.49 Stacked histograms of $\phi$ of the lowest momentum jet in the taggable region . . . . . . . . . . . . . . . . 121

7.50 Stacked histograms of $\phi$ of the second jet in the tag region . . . . 122

7.51 Stacked histograms of $\phi$ of the second jet in the taggable region . 123

7.52 Stacked histograms of $\phi$ of the third jet in the tag region . . . . . 124

7.53 Stacked histograms of $\phi$ of the third jet in the taggable region . . 124

7.54 Stacked histograms of $P_{T}$ of the lepton in the tag region . . . . 125 
7.55 Stacked histograms of $P_{T}$ of the lepton in the taggable region . . 126

8.1 Linearity scan . . . . . . . . . . . . . . . . . . . . . 135

$9.1-2 \ln Q$ for the test hypothesis and null hypothesis. . . . . . . 137

9.2 Fit for $\sigma_{s}+\sigma_{t} \ldots \ldots \ldots \ldots \ldots \ldots \ldots \ldots \ldots \ldots$

9.3 Extraction of limits on $\left|V_{t b}\right|^{2} \ldots \ldots \ldots \ldots \ldots \ldots \ldots$

10.1 CDF combination results . . . . . . . . . . . . . . 141

10.2 Combination $V_{t b} \ldots \ldots \ldots \ldots \ldots \ldots \ldots \ldots \ldots$

10.3 CDF measured cross sections . . . . . . . . . . . . . . 142

10.4 D Ø measured cross sections . . . . . . . . . . . . . . 143

10.5 Predicted CDF combined significance . . . . . . . . . . . . 143

10.6 Extrapolated CDF combined significance. . . . . . . . . . . . . 144

A.1 Mistag matrix plot of $E_{T} \ldots \ldots \ldots \ldots \ldots$. . . . . . 147

A.2 Mistag matrix plot of Number good tracks . . . . . . . . . . 147

A.3 Mistag matrix plot of $\sum E_{T} \ldots \ldots \ldots \ldots \ldots \ldots$

A.4 Mistag matrix plot of $\eta \ldots \ldots \ldots \ldots$. . . . . . . 148

A.5 Mistag matrix plot of the number of primary verticies . . . . 149

A.6 Mistag matrix plot of the number of the $z$ position of the primary

vertex. . . . . . . . . . . . . . . . . . . 149

A.7 Mistag matrix plot of the run number . . . . . . . . . 150

A.8 Mistag matrix plot of the number of luminosity . . . . . . . . 151

A.9 Mistag matrix plot of the bunch luminosity . . . . . . . . . 151

A.10 Mistag matrix plot of the number of jets in the event . . . . . 152

A.11 Mistag matrix plot of error associated with the primary vertex

position in the y direction . . . . . . . . . . . . . . . 152

A.12 Mistag matrix plot of error associated with the primary vertex

position in the $\mathrm{x}$ direction . . . . . . . . . . . . 153

A.13 Mistag matrix plot of the total number of tracks . . . . . . 153

A.14 Mistag matrix plot of $\phi \ldots \ldots$. . . . . . . . . . 154

B.1 $t \bar{t}$ ISR and FSR shape systematics . . . . . . . . . . . . . . 157

B.2 Single-top ISR and FSR shape systematics . . . . . . . . . . . . 158

B.3 Mistag pretag model shape systematic . . . . . . . . . . . . 159

B.4 $Q^{2}$ Shape systematic . . . . . . . . . . . . . . . 160

B.5 ANN shape systematic . . . . . . . . . . . . . . . . 160

B.6 Non- $W$ flavor shape systematic . . . . . . . . . . . . . . 161

B.7 PDF shape systematic . . . . . . . . . . . . . . . . . 161

B.8 $\Delta R$ reweighting systematic $\ldots \ldots \ldots \ldots \ldots \ldots$

B.9 $\Delta R$ reweighting systematic $\ldots \ldots \ldots \ldots \ldots \ldots \ldots$

B.10 $\eta$ jet 2 reweighting systematic . . . . . . . . . . . . 164

B.11 $\eta$ jet 2 reweighting systematic . . . . . . . . . . . . 165

B.12 JES systematic . . . . . . . . . . . . . . . 166

B.13 JES systematic . . . . . . . . . . . . . . . . . 167

B.14 Top mass systematic . . . . . . . . . . . . . . . . 168

B.15 $t \bar{t}$ ISR and FSR shape systematics . . . . . . . . . . . . . 169

B.16 Single-top ISR and FSR shape systematics . . . . . . . . . . . 170

B.17 Mistag pretag model shape systematic . . . . . . . . . . . . . . 171

B.18 $Q^{2}$ Shape systematic . . . . . . . . . . . . . . . . 172

B.19 ANN shape systematic . . . . . . . . . . . . . . . . . . . . . . . . . . . . . . . . . . .

B.20 Non- $W$ flavor shape systematic $\ldots \ldots \ldots \ldots \ldots \ldots . \ldots \ldots$

B.21 PDF shape systematic . . . . . . . . . . . . . . . 173 
B.22 $\eta$ jet 2 reweighting systematic . . . . . . . . . . . . . . . . 174

B.23 $\eta$ jet 2 reweighting systematic . . . . . . . . . . . . . . . . . . . . . . . . . . . . . . . . . . . .

B.24 JES systematic . . . . . . . . . . . . . . . . . 176

B.25 JES systematic . . . . . . . . . . . . . . . . . . . . . . . . . . . . . . . . . . . . . . . . . . . .

B.26 Top mass systematic . . . . . . . . . . . . . 178

C.1 Muon chamber occupancy for normal data taking . . . . . . . . 180

C.2 Muon chamber occupancy with a noisy channel. . . . . . . . . 181

C.3 Muon chamber occupancy with a dead channel. . . . . . . . . 181

C.4 J/ $\Psi$ hit width for CMP chambers. . . . . . . . . . . . . 183

C.5 J/ $\Psi$ hit width for upper CMP chambers. . . . . . . . . . 183

C.6 J/ $\Psi$ hit width for lower CMP chambers. . . . . . . . . . . . 184 


\section{Introduction}

Particle physics is the study of the smallest known constituents of the universe. The goal of particle physics is to study the fundamental building blocks of nature and the interactions between them by testing theories against experimental evidence. One theory in particular has withstood many experimental tests over the course of many decades: the Standard Model of particle physics. Although the Standard Model of particle physics has been enormously successful at describing many of the basic forces and particles in our universe, not all aspects of this model have yet been completely experimentally tested. Thus, the goal of particle physics is to test all aspects of the Standard Model, and to seek new physics processes or areas where the Standard Model fails to accurately predict experimental results.

It is a delicious irony that the study of the smallest constituents of the universe requires some of the world's largest and most complicated experimental apparatus. The Fermilab Tevatron is one such apparatus, with the primary collider ring measuring a kilometer in radius. This large beamline is used to collide protons and anti-protons at very high center of mass energies of $\sqrt{s}=1.96 \mathrm{TeV}$, at the CDF and DØ experiments. This allows the creation of interesting and exotic particles, one of the most fascinating of which is the top quark.

With a mass of approximately $175 \mathrm{GeV} / c^{2}$, about the mass of a gold atom, the top quark is the heaviest known quark. In comparison, the second heaviest known quark, the $b$ quark, has a mass of approximately $5 \mathrm{GeV} / c^{2}$. This large mass suggests that the top quark may play a special role in nature.

Top quarks are usually produced via the strong force. However, electroweak top production, also called single-top production, is also predicted by the Standard Model. Single-top production has not yet been observed, thus searching for single-top quark production is an interesting way to test the Standard Model. Additionally, a measurement of single-top production allows a direct measurement of the CKM matrix element, $V_{t b}$. A direct measurement of $V_{t b}$ will provide constraints on many beyond the Standard Model processes.

Prior to the Winter 2008 CDF results, which will be described in this thesis, both CDF [1] and DØ [2],[3] have observed a $3 \sigma$ evidence for single-top production, but as of the writing of this thesis, there has been no $5 \sigma$ discovery of single-top quark production. 


\section{The Tevatron and the CDF II Detector}

Detecting and measuring heavy, fast decaying particles, such as the top quark, is a difficult proposition. Ordinary matter is composed of relatively few particles: up (u) and down (d) quarks and leptons such as electrons and muons. Most other particles decay relatively quickly into these constituents, and thus are not present at any discernible level in everyday matter. Such particles must therefore be artificially produced. This can be done using a machine called a particle accelerator.

Particle accelerators operate by accelerating a beam of particles to a very high momentum, and then smashing this beam against either a fixed target or another beam of accelerated particles. These energetic collisions create an array of particles, some of which will be more unusual particles such as top quarks.

\subsection{The Fermilab Accelerator Complex}

The Fermilab complex consists of a system of eight accelerators. These accelerators fall into four major categories, the Proton Source, the Anti-proton Source, the Main Injector and Recycler, and the Tevatron. A schematic overview of the Fermilab complex can be see in Figure 2.1.

In the Proton Source, hydrogen gas is converted to H- ions. These ions are then accelerated in the Cockcroft-Walton accelerator to an energy of $750 \mathrm{keV}$. The $\mathrm{H}$ - ions are sent to a linear accelerator (called the Linac) where they are accelerated to $400 \mathrm{MeV}$ using radio frequency (RF) resonators. The $\mathrm{H}-$ ions are stripped of their electrons to create bare protons by passing through a carbon foil. The protons are fed into the booster, which is the first synchrotron in the accelerate chain. In the booster, the protons are accelerated to $8 \mathrm{GeV}$ and then are sent into the Main Injector.

For anti-proton production, the protons are sent into the Main Injector where they are accelerated to $120 \mathrm{GeV}$. This beam is then sent to the anti-proton source, where it hits a nickel target. The resulting particles are then focused into a beam using a lithium lens. They are then sent through a magnet which acts as a charge-mass spectrometer to filter out only anti-protons of energy approximately $8 \mathrm{GeV}$. The resulting anti-protons are sent to the debuncher, where they are spread out by radio frequency manipulations, and then stochastically cooled. The anti-protons are then sent to the accumulator, where they are stored at an energy of $8 \mathrm{GeV}$ and cooled using radio frequency and stochastic 


\section{FERMILAB'S ACCELERATOR CHAIN}

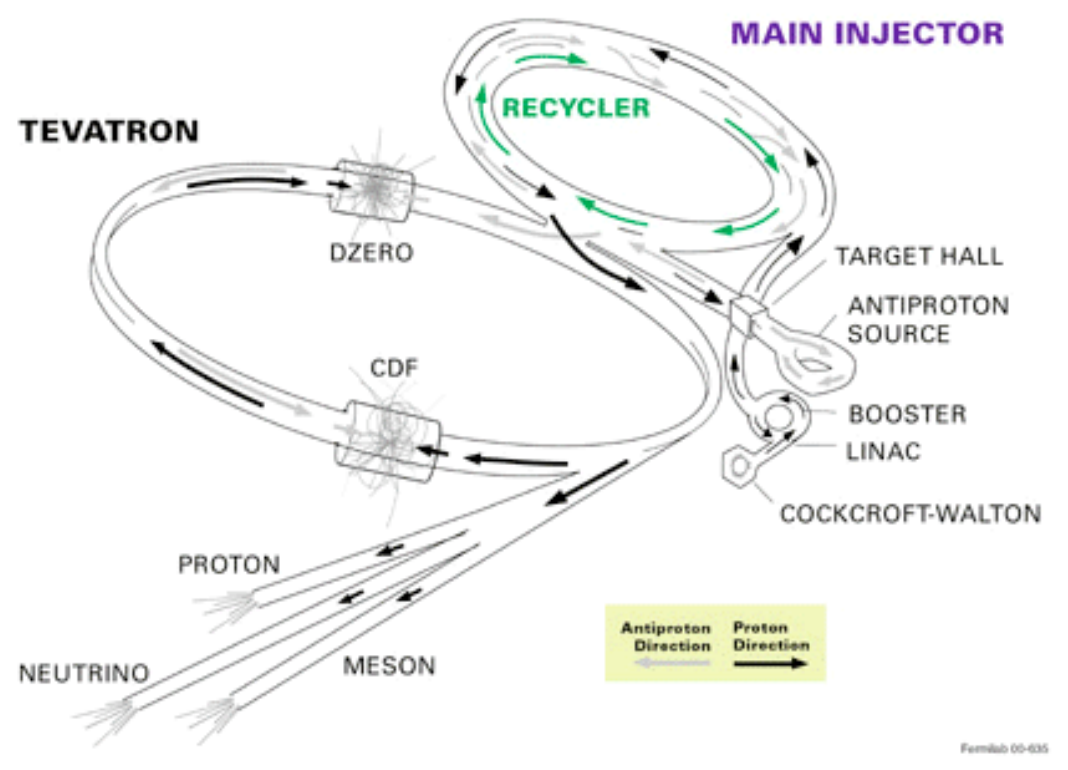

Figure 2.1: Schematic overview of the Fermilab accelerator chain.

cooling.

The anti-protons are sent from the anti-proton source to the recycler. The recycler further cools the anti-proton source using stochastic cooling and electron cooling. For the electron cooling, the anti-proton beam is overlaid with a $4.3 \mathrm{MeV}$ electron beam traveling in the same path as the recycler. Momentum is transfered between the anti-proton and electron beam due to Coulomb scattering, shrinking the phase space and cooling the anti-protons.

Finally, protons and anti-protons are sent to the Tevatron, a superconducting synchrotron. The Tevatron accelerates 36 proton and 36 anti-proton bunches to $\sqrt{s}=1.96 \mathrm{TeV}$. Proton anti-proton collisions occur at two intersection points: $\mathrm{B} 0$, where the CDF detector is located, and $\mathrm{D} \varnothing$, where the $\mathrm{D} \varnothing$ detector is located. At these intersection points, the beam is focused and the beam halo is removed by moving stainless steal collimators close to the beam.

\subsubsection{Luminosity}

The instantaneous luminosity $L$ produced at the Tevatron can be written as follows:

$$
L=n \cdot f \cdot \frac{N_{p} N_{\bar{p}}}{4 \pi \sigma_{x} \sigma_{y}}
$$

Here $n$ is the number of bunches, $f$ is the revolution frequency, $N_{p}$ and $N_{\bar{p}}$ are the number of protons and anti-protons per bunch, and $\sigma_{x}$ and $\sigma_{y}$ are the average width of the bunches. $L$ is measured in units of $\mathrm{cm}^{-2} \mathrm{~s}^{-1}$. Integrated 
luminosity can be calculated by integrating the instantaneous luminosity over time, $L_{i n t}=\int L \mathrm{~d} t$. For a particular process with cross section $\sigma$, the number of events expected is given by $N=\sigma \cdot L_{i n t}$. An integrated luminosity of $2.2 \mathrm{fb}^{-1}$ is used in this analysis, where here $p b=10^{-36} \mathrm{~cm}^{2}$ and $f b^{-1}=1000 b^{-1}$.

\subsection{The CDF Detector}

An accurate measurement of the energy, momentum and particle type of many particles is needed to detect single top production. These particles include leptons such as electrons, muons and neutrinos, and quarks whose final signature is a shower of hadrons such as pions, protons and neutrons. The CDF detector is designed to efficiently detect particles produced in Tevatron collisions. This requires many detector pieces, such as tracking, calorimetry and muon identification chambers. By combining the information from these chambers, most particles can be reconstructed and many particle properties can be measured. For instance, as shown in Figure 2.2, an electron is detected as a track in the tracking chambers followed by a shower in the electromagnetic calorimeter, a proton is detected as a track in the tracking chambers and a shower in the hadronic calorimeter, and a muon would be indicated by a track pointing to a track segment in the muon chambers.

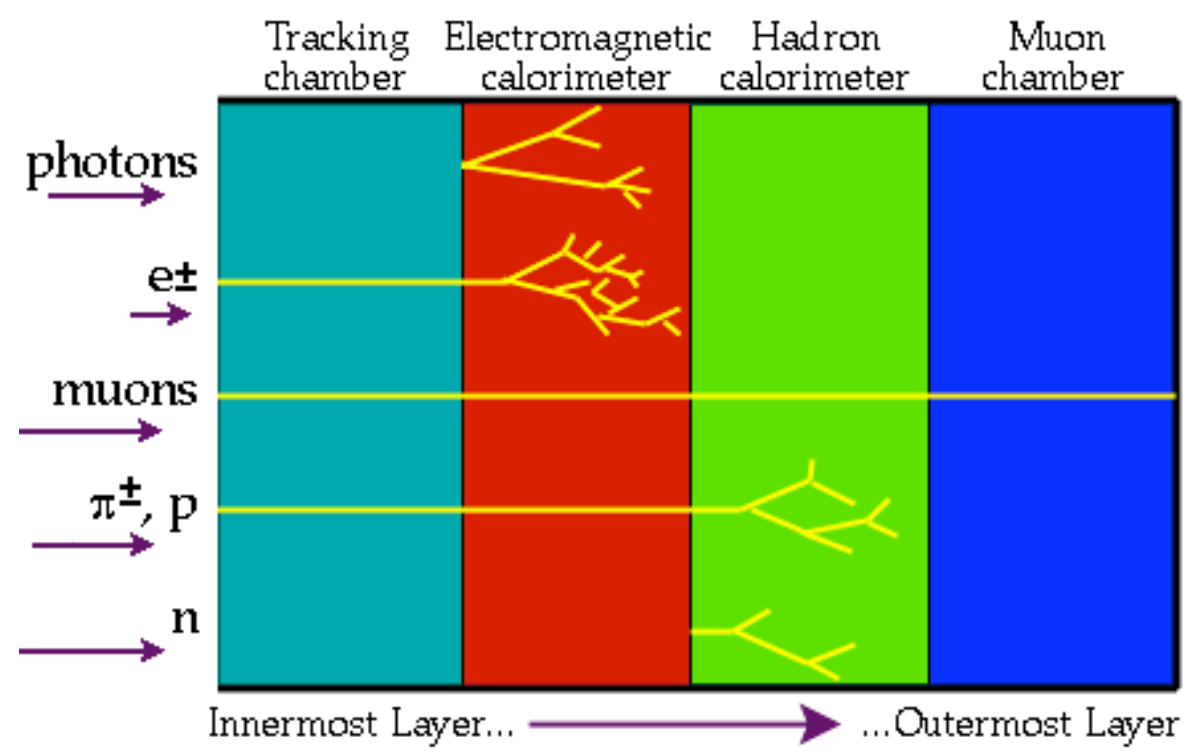

Figure 2.2: Particle detection at CDF.

A cylindrical coordinate system is used to describe the CDF detector. Here, $z$ is defined as the direction along the proton beam, $\theta$ as the polar angle and $\phi$ as the azimuthal angle. The azimuthal angle is defined with respect to the radial direction and the polar angle is defined with respect to the proton beam 
direction. Additionally, the polar angle is often expressed through the pseudorapidity, $\eta$. Here $\eta=-\ln (\tan (\theta / 2)$.

Figure 2.3 is a diagram of the CDF detector. The detector components used in this analysis will be described in more detail below. Additional information on the CDF detector can be found in References [4] through [18].

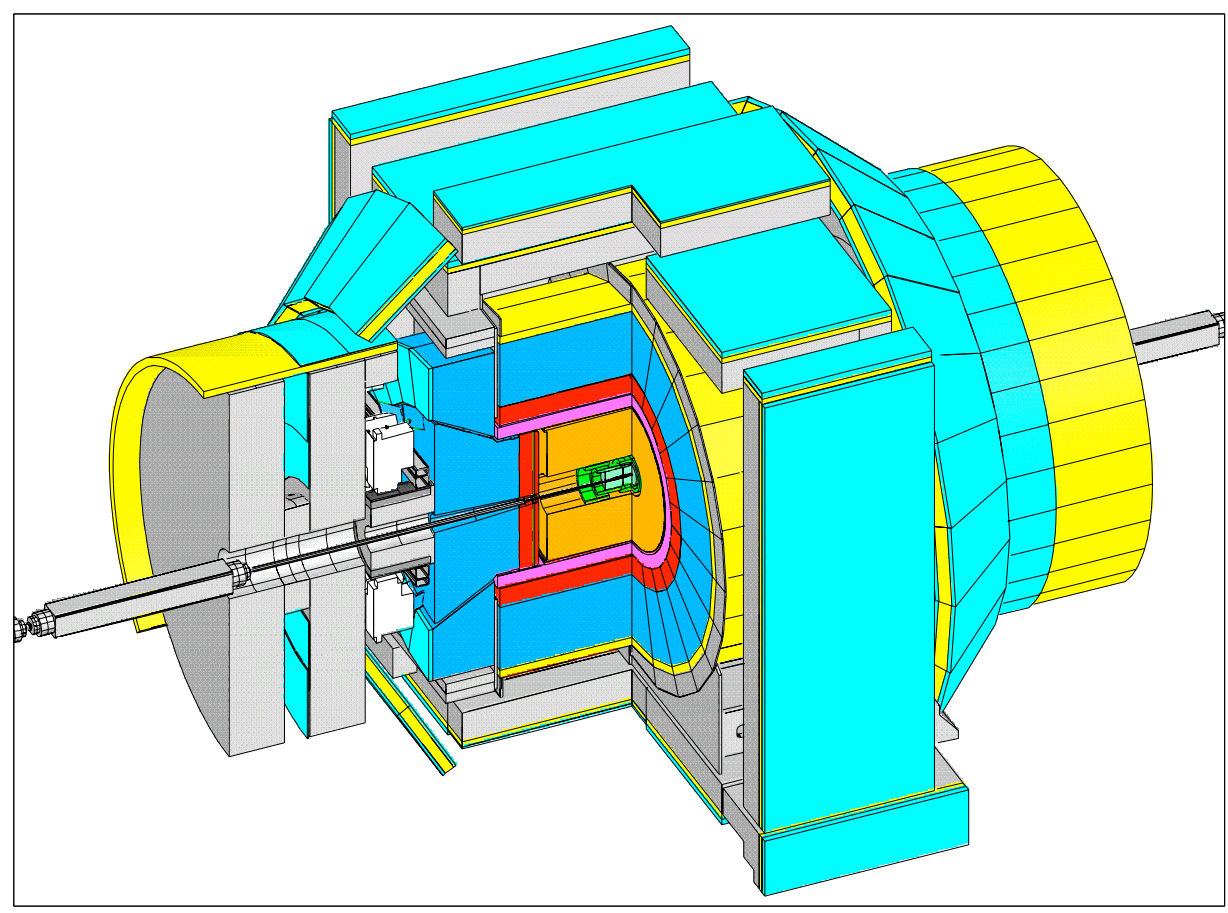

Figure 2.3: The CDF Run II detector

\subsubsection{Tracking}

The innermost layer of tracking consists of the silicon detectors: L00, SVX and ISL. L00 ('layer zero zero') is the innermost layer, consisting of a single-sided, radiation hard silicon microstrip detector immediately outside the beampipe at $r \approx 1.5 \mathrm{~cm}$. L00 serves to improve track measurements and tracking efficiency.

The silicon vertex detector, or SVX, sits outside L00, from $\mathrm{r}=2.5 \mathrm{~cm}$ to $\mathrm{r}=10.7 \mathrm{~cm}$. The SVX is composed of three cylindrical barrels, each built of 5 layers of double-sided silicon microstrip detectors. The SVX has a total length of $96 \mathrm{~cm}$ which covers the region $|\eta|<2$. It is used for high precision tracking and vertex determination. The sensors of all SVX layers feature strips parallel to the $z$ axis on one side, but the strips on the backside of the sensors in layers 0,1 and 3 are rotated by 90 degrees. This provides both a detailed measurement of the coordinates in the $r-\phi$ plane and a precise determination of the $z$ coordinate. Additionally, the strips on the backside of layers 2 and 4 are rotated by 1.2 degrees. This enables a unique determination of the 3 dimensional track. 
The last silicon layer, the ISL or intermediate silicon layer, also consists of a double-sided silicon microstrip detector. The ISL provides enhanced track linking to the tracker (described below) in the central region and silicon-only tracking capability in the plug region. The entire tracking system provides an impact parameter resolution of $40 \mu \mathrm{m}$ including the 30 micrometer beam width [4].

The final component of the tracking system is the Central Outer Tracker (COT). The COT covers the region $|\eta| \leq 1.0$. It is an open cell drift chamber filed with a mixture of argon and ethane gas, built to identify tracks of charged particles. The drift chamber consists of 96 layers, organized into eight alternating axial and $2 \%$ stereo angle superlayers. The COT provides a hit position resolution of about $140 \mu \mathrm{m}$, a momentum resolution $\sigma\left(p_{T}\right) / p_{T}^{2}=0.0015(\mathrm{GeV} / \mathrm{C})^{-1}$, and $\mathrm{dE} / \mathrm{dx}$ information for the tracks.

The tracking layers are contained in a large superconducting solenoid. The solenoid generates a 1.4T magnetic field parallel to the beam axis, and allows the momentum or particles to be measures using the tracking information. A cut away view of the CDF detector showing the location of the tracking chambers is shown in Figure 2.4.

\section{CDF Tracking Volume}

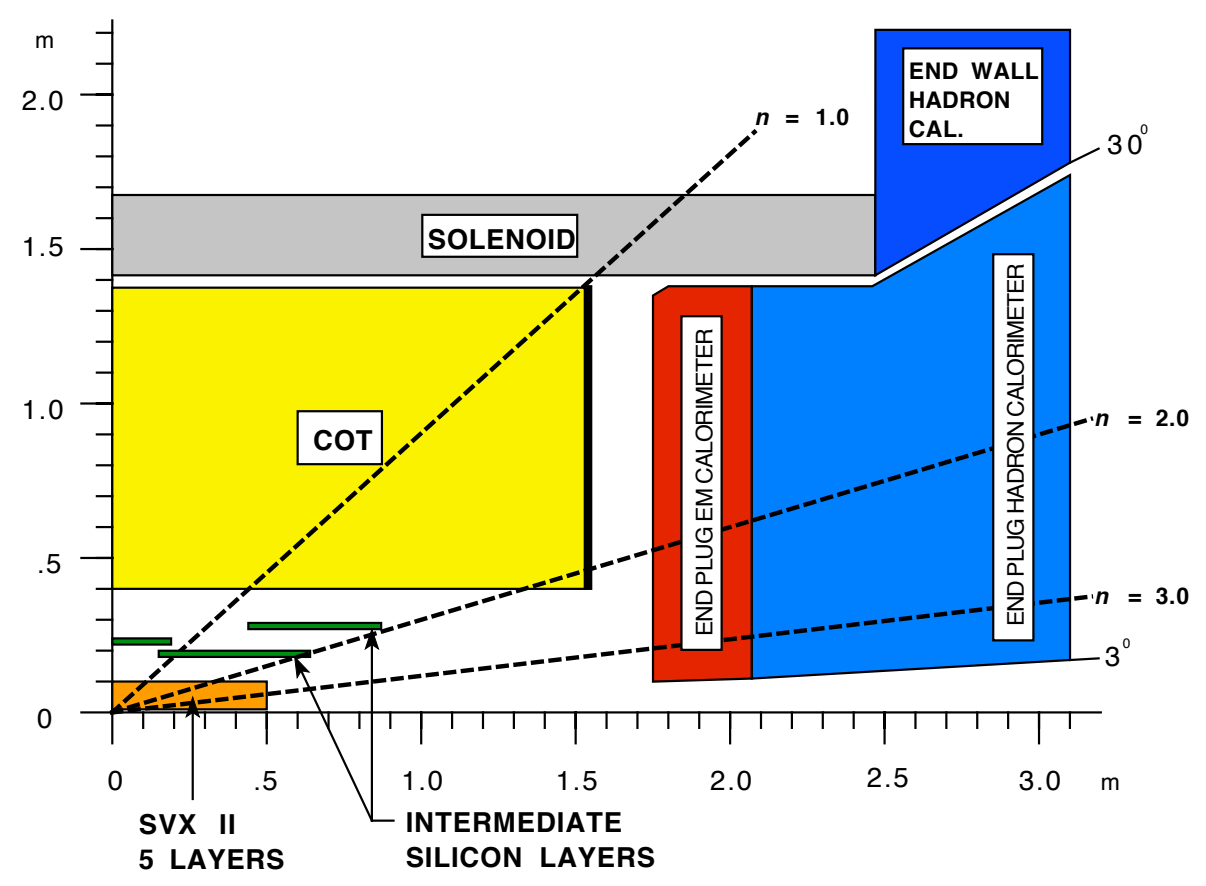

Figure 2.4: The CDF Run II detector cut away view. 


\subsubsection{Calorimeters}

The next detector layer consists of the electromagnetic and hadronic calorimeters. The scintillator-based calorimetry covers the region $|\eta| \leq 3.6$, and measures electron and photon and jet energies. The calorimeters are divided into five regions. The Central Electron Magnetic Calorimeter (CEM) and Central Hadronic Calorimeter (CHA) cover the $\eta$ rate to $|\eta|<1.1$ for CEM and $|\eta|<0.9$ for $\mathrm{CHA}$. The CEM is a sampling calorimeter consisting of 31 layers of polystyrene scintillator interspersed with lead, with a depth of 19 radiation lengths. The CHA consists of 32 layers of acrylic scintillator interspersed with $2.5 \mathrm{~cm}$ of steal absorber, with depth of 4.7 interaction lengths. In addition, the end wall hadronic calorimeter (WHA) and the end-plug electromagnetic calorimeter (PEM) and end-plug hadron calorimeter (PHA) offer a complete coverage in $2 \pi$ up to $|\eta| \leq 3.64$ [4].

\subsubsection{Muon Chambers}

The muon systems surround the outside of the detector, and are designed to identify high energy muons escaping the calorimeters. The muon system consists of a series of scintillators and proportional chambers. The CMU or central muon chamber is a wire proportional chamber, which covers the region $|\eta| \leq 0.6$ and detects muons with $\mathrm{p}_{T}>2.0 \mathrm{GeV} / \mathrm{c}$. The CMP sits outside the CMU in a roughly rectangular configuration. The CMP is behind more material, and thus has a better signal to background ratio than the CMU. It can detect muons with $\mathrm{p}_{T}>3.0 \mathrm{GeV} / \mathrm{c}$. The CMX or central muon extension is also a proportional chamber, and it covers the range $|\eta| \leq 0.6$ to $|\eta| \leq 1.0$. Finally, the BMU or barrel muon chambers cover the region from $1.0 \leq|\eta| \leq 1.5$ [4]. A picture of the $\eta-\phi$ coverage of the CDF muon system is shown in 2.5. 
Figure 1. CDF $\eta-\phi$ Map for Central Muons

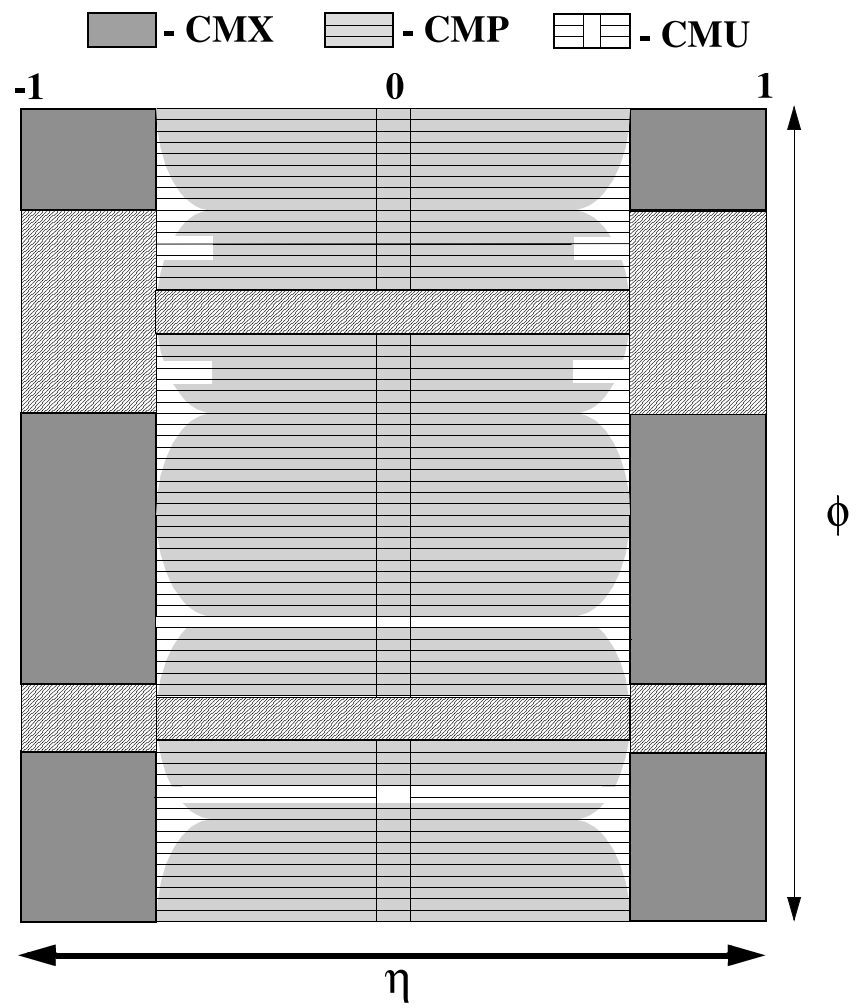

Figure 2.5: Geometrical coverage of the CDF muon chambers, on the $\eta-\phi$ plane. 


\subsection{Trigger System}

The Tevatron creates events at a far faster rate than the rate at which events can be recorded. Thus, an important part of the CDF experiment is the trigger system, which helps decide which events to save. The trigger consists of three main components, called Level 1, Level 2 and Level 3. Level 1 and Level 2 consists of special-purpose hardware, while Level 3 is a linux computer farm. This system was designed to select the most interesting events and reject much of the inelastic background [19], [20].

Level 1 is the first and coarsest trigger. It uses information from the COT, calorimeter and muon systems to accept events based on tracking, calorimeter energy, missing energy and kinematic properties of the event. The eXtremly Fast Trigger (XFT) reconstructs tracks in the transverse plane of the COT. An extrapolator, known as the XTRP, then matches those tracks to electromagnetic calorimeter energy clusters or track segments in the muon systems. Level 1 has an acceptance rate of about $30 \mathrm{KHz}$.

Level 2 gets additional information from sources such as the SVX and the central shower-max detector (CES). The CES is a strip chamber placed in the CEM at the location of the typical shower maximum, designed to improve identification of electrons and photons. Data from the silicon tracker (SVX) is associated with XFT tracks by the Silicon Vertex Tracker (SVT) to provide precise measurements of the track impact parameter. The rate of events accepted by Level 2 is about $300-1000 \mathrm{~Hz}$.

Finally, the events are sent to the Level 3 system, a farm of parallel processors that each analyze a full event readout. This computing farm has access to the full event record. Events that pass the Level 3 triggers are then are sent to permanent storage at a rate of about $75-100 \mathrm{~Hz}$. A diagram of the CDF trigger system can be found in Figure 2.6 .

The triggers used in this analysis include high $p_{T}$ electron and muon triggers. These are described in more detail in Section 4.1. 


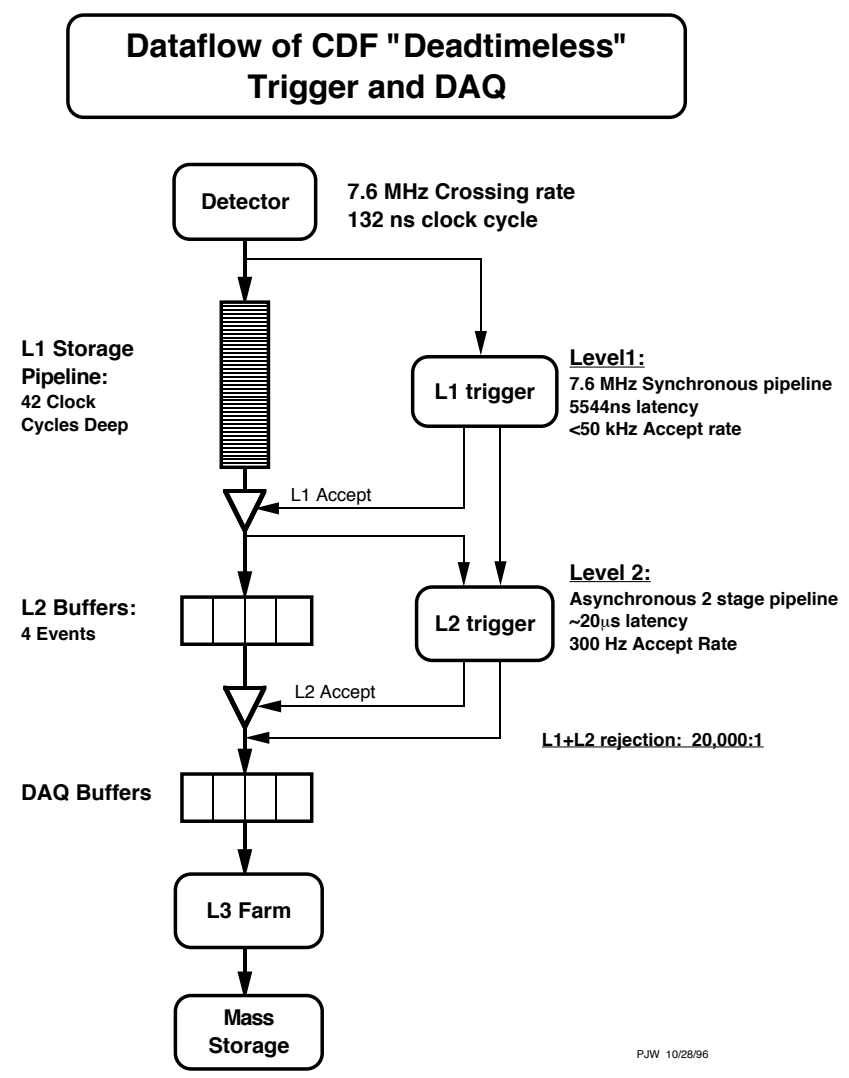

Figure 2.6: Diagram of the CDF trigger system. 


\section{The Standard Model and the Top Quark}

\subsection{The Standard Model of Particle Physics}

The Standard Model of particle physics has been enormously successful at describing many of the basic forces and particles in our universe. The Standard Model is a collection of quantum field theories which describes the known fundamental particles, as well as the strong, weak and electromagnetic forces. The gravitational force is not included in this model.

The state of particles is described by a series of quantum numbers. These numbers describe properties such as baryon number, lepton number, spin, etc. Particles are divided into two groups by their spin: fermions or half integer spin particles, and bosons or integer spin particles.

Fermions are further divided into two groups: quarks which interact via the strong force and leptons which do not. Quarks and leptons are grouped into three generations with each generation containing two quarks and two leptons. Ordinary matter is composed mainly of particles from the first generation, as higher generation particles generally decay quickly into these particles. However, particles from the second and third generations can be created in a high-energy environment. Table 3.1 gives an overview of all Standard Model fermions, including properties such as mass and electric charge.

Quarks and leptons form the building blocks of all known matter. Leptons, such as electrons or neutrinos, can be observed in nature. In contrast, bare quarks have never been directly observed, but rather combine to form hadrons consisting of either three quarks or a quark and anti-quark. For example, a proton is a hadron consisting of two up quarks and one down quark.

Bosons are particles with integer spin. They are responsible for mediating the interactions between particles in the Standard Model. The Standard Model includes three forces: strong, weak and electromagnetic. While these three forces appear distinct at low energies, at higher energies the weak and electromagnetic forces have been shown to be facets of the same electroweak force. This will be discussed in more detail in Section 3.1.1. The electromagnetic force is mediated by the photon, the weak force is mediated by the $W$ and $Z$ bosons and the strong force is mediated by the gluon. Table 3.2 summarizes the Standard Model bosons and their properties.

The electromagnetic interactions between quarks and leptons occur via the exchange of a photon, as described by quantum electrodynamics. Quantum 


\begin{tabular}{|c|c|c|c|}
\hline & \multicolumn{3}{|c|}{ Fundamental Fermions } \\
\hline \hline & \multicolumn{3}{|c|}{ Quarks } \\
\hline Gen & Flavor & Charge & Mass $\left[\mathrm{MeV} / \mathrm{c}^{2}\right]$ \\
I & Up $(u)$ & $+2 / 3$ & 1.3 to 3.0 \\
& Down $(d)$ & $-1 / 3$ & 3 to 7 \\
II & Charm $(c)$ & $+2 / 3$ & $1.25 \pm 0.09 \times 10^{3}$ \\
& Strange $(s)$ & $-1 / 3$ & $95 \pm 25$ \\
III & Top $(t)$ & $+2 / 3$ & $172.6 \pm 1.4 \times 10^{3}$ \\
& Bottom $(b)$ & $-1 / 3$ & $4.2 \pm 0.07 \times 10^{3}$ \\
\hline \hline & \multicolumn{3}{|c|}{ Leptons } \\
Gen & Flavor & Charge & Mass $\left[\mathrm{MeV} / \mathrm{c}^{2}\right]$ \\
I & electron $\left(e^{-}\right)$ & -1 & 0.511 \\
& electron neutrino $\left(\nu_{e}\right)$ & 0 & $<2 \times 10^{-6}$ \\
II & Muon $\left(\mu^{-}\right)$ & -1 & 105.7 \\
III & Muon neutrino $\left(\nu_{\mu}\right)$ & 0 & $<0.019$ \\
& Tau $\left(\tau^{-}\right)$ & -1 & 1777.0 \\
& Tau neutrino $\left(\nu_{\tau}\right)$ & -1 & $<18.2$ \\
\hline
\end{tabular}

Table 3.1: Fundamental fermions of the Standard Model [21]. Top quark mass measurement is best current combined $\mathrm{CDF}$ and $\mathrm{D} \emptyset$ average [22].

\begin{tabular}{|c|c|c|c|}
\hline \multicolumn{4}{|c|}{ Fundamental Bosons } \\
\hline \hline Interaction & Name & Charge $[e]$ & Mass $\left[\mathrm{GeV} / \mathrm{c}^{2}\right]$ \\
Electromagnetic & Photon $(\gamma)$ & $<5 \times 10^{-30}$ & $<6 \times 10^{-17}$ \\
Weak & $W$ boson $( \pm W)$ & \pm 1 & $80.403 \pm 0.029$ \\
Weak & $Z$ boson $(Z)$ & 0 & $91.1876 \pm 0.0021$ \\
Strong & gluon $(g)$ & 0 & 0 \\
\hline
\end{tabular}

Table 3.2: Fundamental bosons of the Standard Model [21].

chromodynamics similarly explains strong interactions via gluon exchange.

\subsubsection{The Weak Force and CKM Mixing}

The weak interaction is mediated by the $W$ and $Z$ bosons. The relatively large mass of these particles means that the weak force acts only over a limited range. However, the weak and electromagnetic forces are actually facets of the same force, the electroweak force. The electroweak theory was proposed by Glashow, Salam and Weinberg [24], [25], [26], who suggested that above a certain energy, the 'unification energy', the weak and electromagnetic forces merge into a single electroweak force. The difference in the observed strengths of the two forces is due to electroweak symmetry breaking. The currently favored method for this electroweak symmetry breaking is the Higgs mechanism [27]. In addition to ascribing masses to the gauge bosons ( $W$ and $Z$ ), the Higgs mechanism also gives masses to the quarks and leptons, by their coupling to the scalar quantum field. The quantum excitation of the Higgs field is known as the Higgs boson. While the electroweak theory has been verified by experiments with high precision, the 
Higgs boson has not been observed and the search for the Higgs boson is in fact an exciting area of research.

Quark mixing in the Standard Model can be seen to arise from the Yukawa interactions with the Higgs condensate, which couples the left handed quark doublets, $Q_{L}$ with the right handed quark singlets $\left(d_{R}\right.$ and $\left.u_{R}\right)$. The Yukawa term in the Lagrangian is:

$$
\mathcal{L}=-Y_{i j}^{d} \overline{Q_{L i}} \phi d_{R j}-Y_{i j}^{u} \overline{Q_{L i}} \epsilon \phi^{*} u_{R j}+\text { h.c. }
$$

Here, $Y$ is a $3 \times 3$ complex matrix, $\phi$ is the higgs field, $i, j$ indicate quark generations and $\epsilon$ is the $2 \times 2$ antisymmetric tensor. Equation 3.1 yields the quark mass terms when the higgs field, $\phi$, acquires a vacuum expectation value of $\langle\phi\rangle=(0, v / \sqrt{2})$. The physical states can be obtained by diagonalizing $Y$ using four unitary matrices, i.e. $M_{\text {diag }}^{f}=V_{L}^{f} Y^{f} V_{R}^{f \dagger}(v / \sqrt{2})$ where $f=u, d$. It can then be shown that the charged current $W^{ \pm}$interactions couple to the physical $u_{L j}$ and $d_{L k}$ states with strengths given by a $3 \times 3$ unitary matrix, known as the Cabibbo-Kobayashi-Maskawa (CKM) matrix [21]. A derivation can be found in Appendix D as well as in Reference [28].

The physical consequence of this coupling is that the weak force allows transitions between quarks of different generations. This is known as CabibboKobayashi-Maskawa (CKM) mixing. The CKM matrix is shown in Equation 3.2. Elements of the matrix describe the strength of weak interaction vertex for any two quarks. For example, the decay rate of $d \rightarrow u+W^{-}$is proportional to $\left|V_{u d}\right|^{2}$. Although it is possible to have transitions between all generations via the weak force, transitions within generations are favored since the diagonal elements of the CKM matrix are larger than the off-diagonal elements.

$$
\left(\begin{array}{c}
d^{\prime} \\
s^{\prime} \\
b^{\prime}
\end{array}\right)=\left(\begin{array}{ccc}
V_{u d} & V_{u s} & V_{b u} \\
V_{c d} & V_{c s} & V_{c b} \\
V_{t d} & V_{t s} & V_{t b}
\end{array}\right)\left(\begin{array}{c}
d \\
s \\
b
\end{array}\right)
$$

The current limits and measurements of the magnitude of the elements of the CKM matrix, assuming unitarity, are given below, as found in [21]. Here, singletop production measurements of $V_{t b}$ are not included:

$$
\left(\begin{array}{ccc}
0.97418 \pm 0.00027 & 0.2255 \pm 0.0019 & (4.31 \pm 0.30) \times 10^{-3} \\
0.230 \pm 0.011 & 0.957 \pm 0.017 & (41.6 \pm 0.6) \times 10^{-3} \\
(7.4 \pm 0.8) \times 10^{-3} & (40.6 \pm 2.7) \times 10^{-3} & >0.9
\end{array}\right)
$$

\subsubsection{Parton Distribution Functions}

This analysis is not truly carried out at the quark level. Instead, the data events originate from collisions of composite particles (in this case protons and anti- 
protons). To properly model processes in such a case, the dynamics of the full particle must be considered. Specifically, the momentum of the proton or antiproton is shared among the elementary constituent particles contained in the hadron, the quarks and gluons (often referred to as "partons" in this context). The fraction of momentum $(x)$ carried by an individual parton is characterized by the parton distribution functions or PDFs. Here the probability of finding a certain parton with a momentum fraction between $\mathrm{x}$ and $\mathrm{x}+\mathrm{dx}$ is given by $\mathrm{f}(\mathrm{x}) \mathrm{dx}$. Figure 3.1 shows the PDFs for up, down, and bottom quarks in a proton. This function depends on a scale $\left(Q^{2}\right)$ which describes the energy scale of the interaction. For top-quark physics, this scale is often set to the top-quark mass. Several sets of PDFs are currently available; this analysis uses the CTEQ5L PDFs [29].

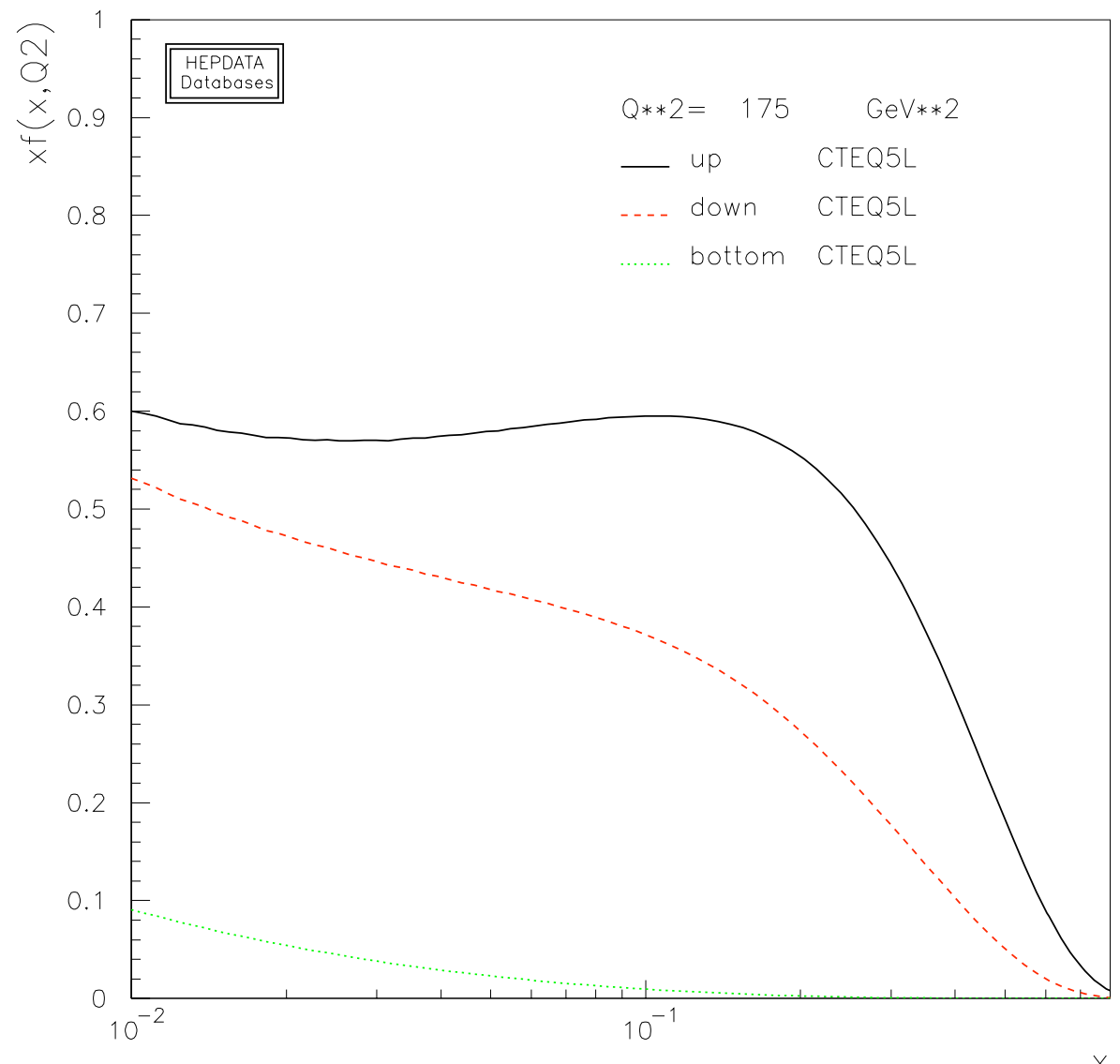

Figure 3.1: Parton distribution functions for the up, down and bottom quarks [30]. These plots were made using the CETQ5L PDFs [29], with $Q^{2}$ set to a top quark mass of $175 \mathrm{GeV} / \mathrm{c}^{2}$. 


\subsection{The Top Quark}

The top quark was discovered at Fermilab in 1995 by CDF [31] and DØ collaborations [32]. The top quark is the heaviest quark in the SM with a mass $m_{t}=172.6 \pm 1.4 \mathrm{GeV} / \mathrm{C}^{2}[22]$, based on the latest combined $\mathrm{CDF}$ and $\mathrm{D} \varnothing$ results. This large mass puts the top quark uniquely near the realm of electroweak symmetry breaking characterized by the Fermi scale of $v=246 \mathrm{GeV}$ [23].

The top quark decays exclusively via the electroweak interaction. At a top mass of $160 \mathrm{GeV} / \mathrm{c}^{2}$, the top quark width has a Standard Model value of $\Gamma=$ $1.02 \mathrm{GeV} / \mathrm{c}^{2}$ and at $m_{t}=180 \mathrm{GeV} / \mathrm{c}^{2}, \Gamma=1.56 \mathrm{GeV} / \mathrm{c}^{2}$. This leads to a very short lifetime of $\approx 0.5 \cdot 10^{-24} \mathrm{~S}[21]$.

The top quark lifetime is so short that top quarks decay before hadronization occurs. This means that the top quark can be thought to basically exist as a free quark, passing its information to its decay products. Since the top quark spin is transfered to the daughter particles, it can be reconstructed from the angular distributions of its decay products [35]. This feature is exploited in single top searches, and will be discussed more in Section 3.3.1.

The top quark decays electroweakly. Since the CKM matrix element $V_{t b}$ is nearly unity, according to three generation unitarity (see Section 3.3.2), the top quark nearly always decays to a $b$ quark and a $W$ boson, which subsequently decays into a lepton and neutrino, or a quark anti-quark pair.

\subsubsection{Top Quark Production via the Strong Interaction}

At the Tevatron, top quarks are mostly produced via the strong interaction. This proceeds either by $q \bar{q}$ annihilation (85\%) or gluon fusion (15\%)[21]. Figure 3.2 illustrates these two modes of top quark production. The cross sec-

tion for Tevatron $t \bar{t}$ production at $1.96 \mathrm{TeV}$ is $7.3 \pm 0.5$ (stat) \pm 0.6 (syst) \pm 0.4 (lumi)pb, according to the latest CDF Run II measurment given a top mass of $175 \mathrm{GeV} / \mathrm{c}^{2}$ [33]. 

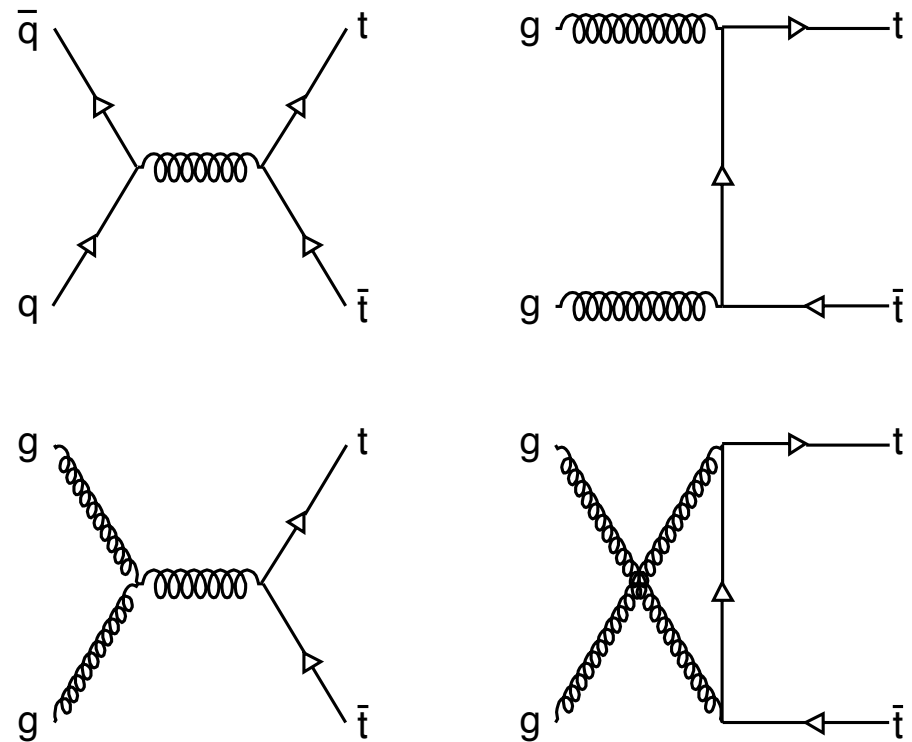

Figure 3.2: Top-quark production via the strong interaction. Diagrams include $q \bar{q}$ annihilation (top left) and gluon fusion (top right, bottom left and bottom right). 


\subsection{Single-Top Quark Production}

In contrast to the $t \bar{t}$ production mode, top quarks can also be produced by the weak interaction. This is called 'single-top' production. There are two main modes of single-top production expected to be observed at the Tevatron: $t$ channel or $W$-gluon fusion, and $s$-channel or $W^{*}$ production. A third mode, $t W$ production, is kinematically suppressed at the Tevatron and not considered in this analysis, but will be of interested at the CERN LHC [36]. Expected single-top $t$-channel and $s$-channel cross sections for the Tevatron are given in Table 3.3 [37], [38].

\begin{tabular}{|c|c|c|}
\hline Process & $t$-channel & $s$-channel \\
\hline \hline$\sigma(p b)$ & $1.98 \pm 0.25$ & $0.88 \pm 0.11$ \\
\hline
\end{tabular}

Table 3.3: Cross sections for single-top production at the Tevatron at $\sqrt{s}=1.96$ $\mathrm{TeV}$ and $M_{t o p}=175 \mathrm{GeV} / \mathrm{c}^{2}$.

Single-top quark production via the $t$-channel consists of a $W$ boson striking a $b$ sea-quark inside the proton or anti-proton. The leading order $2 \rightarrow 2$ process is shown in Figure 3.3. The most important next-to-leading order diagram is a $2 \rightarrow 3$ process taking into account that the $b$ quark originally comes from gluon splitting, as shown in Figure 3.3. This second $b$ quark is generally produced at high angular rapidity and low transverse momentum [39], [40]. The predicted cross section for $t$-channel single-top quark production at the Tevatron is shown in Table 3.3.

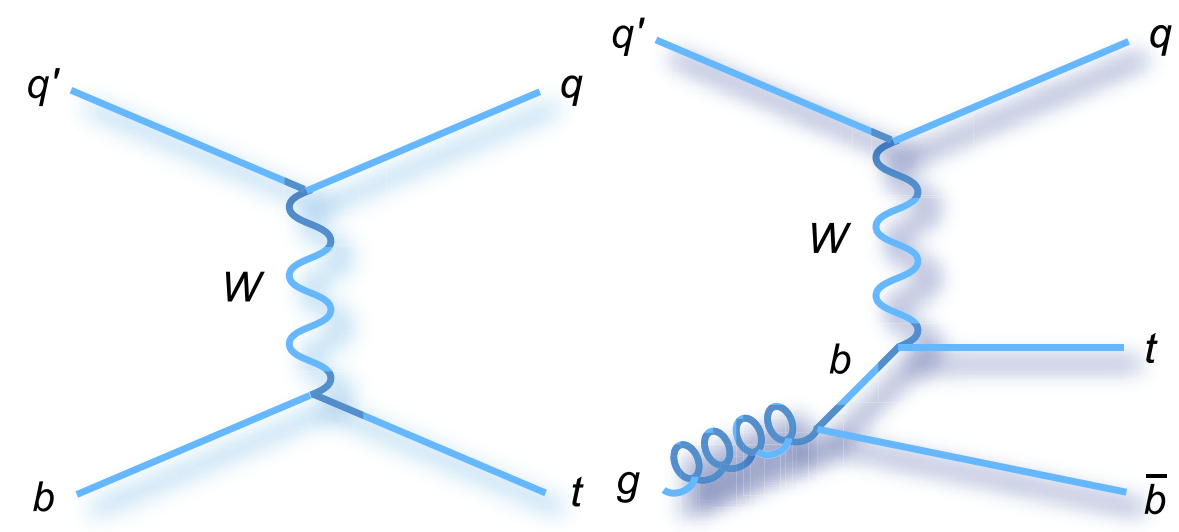

Figure 3.3: $t$-channel single-top production diagrams. The leading order (left), and an example next to leading order diagram (right) are shown

Single top quark production via the $s$-channel consists of two quarks which annihilate into a virtual $W$ boson to produce a t and $\bar{b}$ quark (or $\bar{t}$ and b). The predicted cross section for $s$-channel production is shown in Table 3.3, and a Feynman diagram of the process is given in Figure 3.4.

The multivariate likelihood function analysis described in this thesis is a 


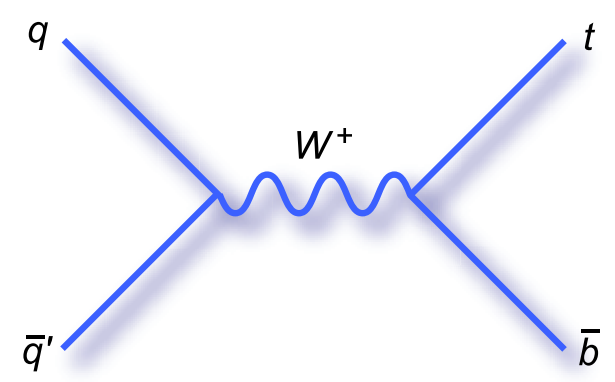

Figure 3.4: $s$-channel single-top production diagrams.

combined search for $s$ and $t$ single-top production at the Tevatron. Since the $s$ channel has a significantly smaller cross section, the optimization of this analysis focuses primarily on $t$-channel single-top kinematics. Separate $s$-channel only analysis are also being completed at CDF and these will be discussed briefly in the outlook portion of this thesis (Section 10.1).

\subsubsection{Single-Top Quark Polarization}

Single-top quark production provides a source of polarized top quarks with very large net polarization [40]. In the short term, this polarization provides a distinct kinematic signature which can be exploited in a multivariate analysis. In the slightly longer term, studying the polarization of these single-top events is an excellent test of the Standard Model weak interaction of the top quark.

As explained in Reference [40], the $W$ boson couples almost exclusively fermions with left-handed chirality. This causes the top quark produced in single-top production to be polarized so that the quarks spin points in the direction of the $d$-type quark. In $s$-channel production, $98 \%$ of the top quarks have their spins in the antiproton direction, coming from the case where the down-type quark is donated by the antiproton. For $t$-channel single-top production, $96 \%$ of top quarks have spins in the direction of the light flavor, recoil quark. The angular variable created by plotting the cosine of the angle between the direction of the final state lepton, and the $d$ type quark then reflects the polarization of the top quark and provides good differentiation between single-top and background production modes [40], [41], [42]. In this analysis, the $t$-channel polarization variable is used, i.e. the cosine of the angle between the light flavor quark and the lepton. This kinematic variable, $\cos \left(\theta_{\text {tchan }}\right)$, is shown in Figure 3.5. 


\section{CDF Run II Preliminary, $2.2 \mathrm{fb}^{-1}$}

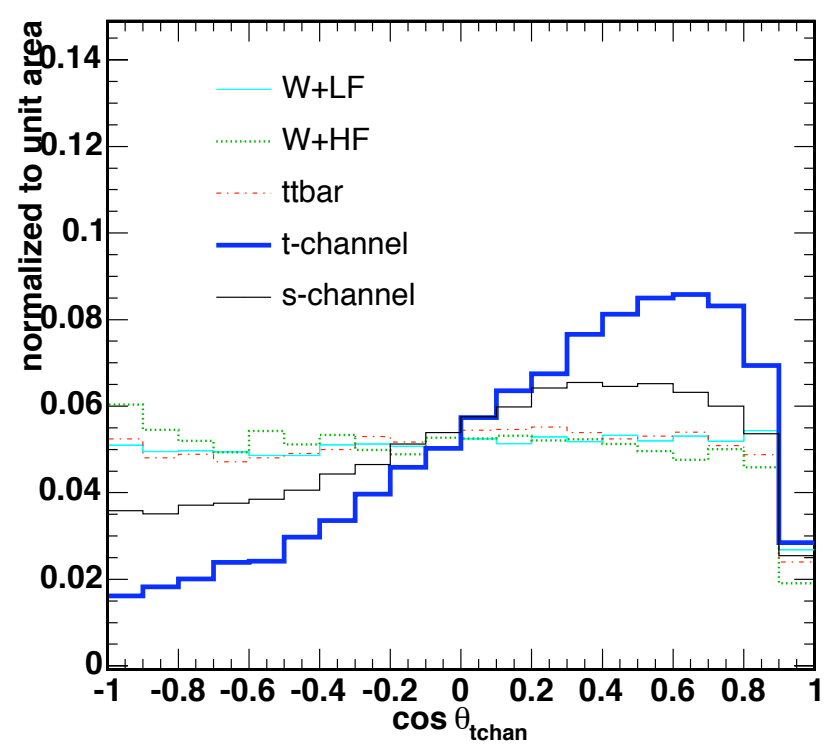

Figure 3.5: Template histogram of $\cos \theta_{t-\text { chan }}$, the cosine of the angle between the light flavor quark and the lepton. Templates are shown for single top (tchannel and s-channel) as well as several major backgrounds (W+LF, W+HF and $t \bar{t}$ ), using the Monte Carlo Model described in Chapter 5. 


\subsection{2 $V_{t b}$ and Single-Top Production}

Prior to the observation of single-top production, measurements of $\left|V_{t b}\right|$ can only be made indirectly. For instance, limits were set on $\left|V_{t b}\right|$ using the ratio of the branching fraction given by:

$$
\mathrm{R}=\mathcal{B}(t \rightarrow W b) / \Sigma_{q=d, s, b} \mathcal{B}(t \rightarrow W q)=\frac{\left|V_{t b}\right|^{2}}{\left|V_{t b}\right|^{2}+\left|V_{t s}\right|^{2}+\left|V_{t d}\right|^{2}}
$$

Assuming the unitarity of the CKM matrix, then $\mathrm{R}=\left|V_{t b}\right|^{2}$. Both $\mathrm{D} \varnothing$ and CDF

have made measurements of $\mathrm{R}$, giving $\mathrm{R}=1.12_{-0.23}^{+0.27}$ [43] and $\mathrm{R}=0.97_{-0.84}^{+0.09}$ [44]. The strongest $95 \%$ CL lower limit on $V_{t b}$ is given by $V_{t b}>0.89$ from the D $\varnothing$ calculation [44].

The CKM matrix element $V_{t b}$ can be quite tightly constrained using the measured values of other CKM matrix elements, given in Table 3.1.1. Assuming there are only three generations of quarks and applying a unitarity constraint on the CKM matrix, it can be shown that $\left|V_{t b}\right|$ should be very close to unity $\left(0.9991_{-0.000004}^{+0.000034}\right)[21]$.

While unitarity of the CKM matrix is well supported by experimental evidence, primarily precise direct experimental measurements of many CKM matrix elements, it remains desirable to make a more direct measurement of $\left|V_{t b}\right|$. The single-top cross section is directly proportional to $\left|V_{t b}\right|^{2}$, so a measurement of single-top would provide a direct measurement of $\left|V_{t b}\right|$, relying only on the assumption that $\left|V_{t b}\right|>>V_{t s}$ and $V_{t d}$. Since it does not depend on the unitarity of the CKM matrix, such a measurement would be a good test of this unitarity, and could also provide a useful test for a fourth quark generation, or other non-Standard Model behavior.

A recent article [45] discussed models for which the current constraints on the CKM matrix could be explained while allowing deviations of $V_{t b}$ from unity. This article explored several minimal extensions of the Standard Model. Two models were proposed, a vector-like top singlet, and a complete new fourth generation. Both these cases introduce additional elements to the CKM matrix, preserving the unitarity of the first two rows of the matrix, but allowing $V_{t b}$ to have values different from one. Parameters for these models are constrained by various experimental results and theoretical considerations. These models have also been tested by an explicit Tevatron search for $t^{\prime}$ quarks [46]. However, measurement of single-top production could provide more stringent constraints on such models.

\subsubsection{Single-top as a Test of New Physics}

Single-top production is an important background for Higgs boson searches and searches for new particles. In addition, the single-top production cross section is sensitive to physics beyond the SM such as new particles and modifications to top quark interactions [47]. 
A measurement of single-top production could probe a variety of new phenomena. Many such models have been proposed. For instance, a new charged vector $W^{\prime}$ boson could increase the measured single-top cross section [49], as could anomalous Wtb couplings [50], [51] and flavor changing neutral currents [52]-[56]. Extra dimension theories, such as Kaluza-Klein excitations of the $W$-boson also predict altered single-top cross sections, such as a decrease in the $s$-channel single-top production rate [57].

Several analyses have been completed directly searching for these types of new physics signatures using a single-top type search. Both CDF and D $\varnothing$ have searched for a $W^{\prime}$ boson decaying to a top quark and bottom quark [60], [61]. Limits on the $W^{\prime}$ mass for the CDF search, which has the current best limits, are given in Figures 3.6. DØ has also completed a search for single-top quarks decaying to flavor changing neutral currents using $230 p b^{-1}$, finding no deviation from the Standard Model predictions [62].

95\% C.L. Observed Limit - CDF Run II Preliminary: $1.9 \mathrm{fb}^{-1}$

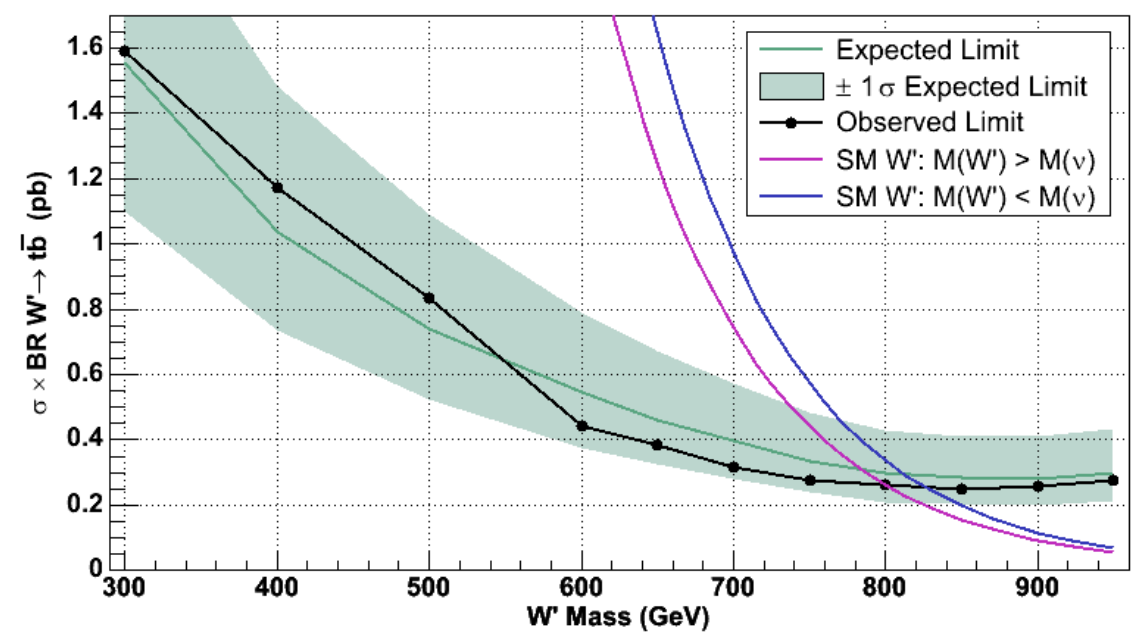

Figure 3.6: Limits on the $W^{\prime}$ mass from CDF [61].

As mentioned, the focus of this analysis is a combined search for both the $t$ channel and $s$-channel modes of single-top production. However, the two modes of single-top production have different sensitivities to physics beyond the Standard Model, making separate $s$ and $t$ channel searches an interesting extension to this analysis.

For instance, the $s$-channel is far more sensitive to extra gauge and scalar bosons than the $t$-channel. The $s$-channel exhibits the possibility of resonant production via extra gauge or scalar bosons, which could lead to an enhancement of the $s$-channel production rate. In contrast, the sensitivity of the $t$ channel to extra gauge and scalar bosons is suppressed, since this process requires virtual exchange of the particle with a heavy mass. Thus, observation of an enhancement of $s$-channel production and no enhancement of $t$-channel 
production would be an indication of possible new gauge or scalar bosons. Similarly, $t$-channel production is more sensitive to modified top quark interactions, like flavor changing neutral currents. An observation of higher than expected rate of $t$-channel production but standard $s$-channel production would be an indication of modified top quark interactions [47].

\subsubsection{Beyond Single-Top}

Finally, the multivariate methods developed in the search for single-top are very applicable for other searches. Some of the main challenges of single-top quark discovery include separating out a small signal from large backgrounds, understanding and modeling complicated event kinematics using Monte Carlo, and quantifying systematics in both the rate of single-top production and the kinematics associated with the events. Techniques developed to deal with such issues are very applicable for other searches, like Higgs searches, at the Tevatron, and beyond. 


\section{Event Selection and
Particle Reconstruction}

The event selection used in this analysis was designed to maximize the amount of single top signal and minimize the amount of background in the final data sample. A typical single top event consists of a top quark and an additional quark, either a bottom quark for s-channel production or a light quark for tchannel production. The top quark decays into a bottom quark and $\mathrm{W}$ boson, which in turn decays into a lepton and neutrino. For t-channel single top quark production, an additional bottom quark is produced at high $\eta$ which nearly always escapes the fiducial region of the CDF detectors. Thus, the single top event signature consists of a lepton, missing energy, and two or three jets where at least one jet must be identified as a $b$ jet.

\subsection{Triggers}

The four triggers used in the single top analysis are designed to select events with high $p_{T}$ lepton candidates. These include central and forward lepton triggers as well as central and CMX muon triggers. The trigger requirements are discussed below. The basic trigger structure is defined in Section 2.3.

\subsubsection{Central Electron Triggers}

The central electron trigger used is ELECTRON_CENTRAL_18. At Level 1, this trigger requires at least one calorimeter tower with $E_{T}>8 \mathrm{GeV}$ and a ratio of energy measured in the hadronic and electromagnetic calorimeters $E_{h a d} / E_{e m}<0.125$. At least one Central Outer Tracker (COT) track with an eXtremly Fast Trigger (XFT) track with $p_{T}>8 \mathrm{GeV}$ with hits in at least four XFT layers is required.

At Level 2, a calorimeter cluster algorithm is run to identify a CEM energy cluster. This cluster is required to have $E_{T}<18 \mathrm{GeV}$ and $E_{\text {had }} / E_{\text {em }}<0.125$, $|\eta|<1.317$ as well as having an XFT track with $p_{T}>8 \mathrm{GeV}$ with hits in at least four XFT layers and stereo confirmation.

Finally, at Level 3 this high $p_{T}$ CEM trigger requires a COT track with $p_{T}>9 \mathrm{GeV} / \mathrm{c}$ matched to an energy cluster in the CEM with $E_{T}>18 \mathrm{GeV}$, with $E_{\text {had }} / E_{e m}<0.125$. The shower profile must also be consistent with the expected shower profile as measured with test-beam electrons. 


\subsubsection{Plug Electron Triggers}

The MET_PEM trigger is used for the selection of forward electron candidates from $W$ decays. At Level 1, this trigger requires at least one forward calorimeter tower with $E_{T}>8 \mathrm{GeV}$ and a ratio of energy measured in the hadronic and electromagnetic calorimeters $E_{\text {had }} / E_{\text {em }}<0.125$ and $E_{\text {had }} / E_{\text {em }}$ measured in the forward region, $E_{\text {had }} / E_{e m}<0.0 .0625$. Also, $\left|E_{T}\right|>15 \mathrm{GeV}$.

At Level 2, this trigger requires an electromagnetic object with $1.1<|\eta|<3.6$, $E_{T}>20 \mathrm{GeV}$ and $E_{\text {had }} / E_{\text {em }}>0.125$ as well as an isolation requirement. At Level 3, the final requirements include $\left|E_{T}\right|>15 \mathrm{GeV}$, an energy deposit of at least $20 \mathrm{GeV}$ in the PEM detector, and a ratio of hadronic to electromagnetic energy $\left(E_{H A D} / E_{E M}\right)>0.125$.

\subsubsection{Central Muon Triggers}

The Central (CMUP) trigger used is MUON_CMUP18 and/or MUON_CMUP18_L2_PT15 depending on the run number of the event. At Level 1, both require matched hits in the CMU detector and a COT track with $P_{T}>4.09 \mathrm{GeV} / \mathrm{c}$. At Level 2 , the COT track is required to have $P_{T}>14.77 \mathrm{GeV} / \mathrm{c}$ matched to hits in the muon chambers. Finally, at Level 3 a COT track with $P_{T}>18 \mathrm{GeV} / \mathrm{c}$ is required to be matched to a segment in the Central Muon Chamber(CMU) and Central Muon Upgrade (CMP). In later run ranges (run number > 226196), a high $p_{T}$ stereo XFT confirmation for the COT track was required as part of the Level 2 trigger(MUON_CMUP18).

\subsubsection{Muon Triggers}

The Central Muon Extension (CMX) muon triggers, such as MUON_CMX18 and MUON_CMX18_L2_PT15 require very similar trigger requirements to the CMUP triggers. At Level 1, both this trigger requires hits in the CMX detector and a COT track with $P_{T}>8.34 \mathrm{GeV} / \mathrm{c}$. At Level 2 , the COT track is required to have $P_{T}>14.77 \mathrm{GeV} / \mathrm{c}$ matched to hits in the muon chambers. Finally, at Level $3 \mathrm{a}$ COT track with $P_{T}>18 \mathrm{GeV} / \mathrm{c}$ matched to a segment in the CMX chambers is required. For certain run ranges these triggers have various secondary requirements which are necessary to control trigger rates with increasing luminosity. For run numbers greater than 222883, the CMX trigger was only enabled for luminosity less than $200 \times 10^{30} / \mathrm{cm}^{2} / \mathrm{s}$ (Muon_CMX18_L2_PT15_LUMI_200). For run numbers greater than 226196, a stereo confirmation (where the Level 2 trigger required a stereo XFT confirmation of the COT track) was added to the trigger (MUON_CMX18_\&_JET10), and the luminosity requirement was dropped. An additional requirement that the luminosity be less than $270 \times 10^{30} / \mathrm{cm}^{2} / \mathrm{s}$ was added for run numbers $>233108$ (MUON_CMX18_\&_JET10_LUM_270) and after run 236132 a dynamic prescale was used instead of the luminosity requirement (MUON_CMX18_\&_JET10_DPS). 


\subsubsection{Data Samples}

The data are broken into various datasets according to the run number and the active triggers. The data were given CDF internal designations in which bhel datasets contain central electrons, bhmu datasets contain muons, and bpel datasets contain forward electrons. A good run list was used to verify that detector components were functional for data. In this analysis, version 19 of the good run list was used, requiring the entire tracking system including silicon trackers, calorimetry and muon chambers to be operational. A total integrated luminosity of $2.2 \pm 0.1 \mathrm{fb}^{-1}$ was used in this analysis.

The data was processed offline. This process involved checking and readjusting for calibrations, correcting for the alignments of the silicon detector, refitting tracks and checking cluster energies, identifying leptons, and performing jet clustering and secondary vertex fits. Two reprocessing versions are used in this analysis. Data from February 2002 to August 2004 was processed with CDFSOFT2 [68] version 5.3.1. Later versions, from December 2004 until August 2007 was processed with CDFSOFT2 version 6.1.1.

\subsection{Event Reconstruction}

For single top events, the expected event signature is a lepton, missing energy which is reconstructed as a neutrino, and two or three jets, at least one of which is identified as a b-jet. Most of the detector components are utilized in the event reconstruction, including both central and forward calorimeters, tracking detectors, including silicon tracking and muon systems. Each of the objects is identified using selection cuts on detector quantities.

\subsubsection{Central (CEM) Electrons}

The largest acceptance in the single top event selection comes from the Central Electromagnetic Calorimeter (CEM). These electron candidates consist of electromagnetic clusters measured using the CEM and Central Electromagnetic Shower Maximum detectors (CES), and associated with tracks from the Central Outer tracker (COT). Selection cuts are made using a variety of energy and track related variables. The variables used are as follows:

- Fiducial: Events must shower in the CEM.

- $E_{T}$ : The transverse electromagnetic cluster energy deposited in the CEM. Defined as the electron magnetic cluster energy $\times \sin (\theta)$ where $\theta$ is the polar angle of the line given by the best COT track pointing the electromagnetic cluster.

- Track $\left|z_{0}\right|:$ The intersection point of the COT track and the beam in the $r / z$ plane. 
- $p_{\mathrm{T}}^{\mathrm{trk}}$ : The transverse momentum of the COT track, measured using the track curvature in the magnetic field.

- Number of Axial and Stereo COT segments: The number of axial and stereo COT super layers with hits pointing to the COT track associated with each electromagnetic cluster.

- $E_{\text {had }}$ : The energy measured in the Hadronic Calorimeter.

- $E_{e m}$ : The energy measured in the Electromagnetic Calorimeter.

- Isolation: The ratio of energy deposited in a cone of radius $\Delta R=0.4$ to the energy of the electromagnetic cluster. (here $\Delta R=\sqrt{\left((\Delta \eta)^{2}+(\Delta \phi)^{2}\right.}$ ).

- $L_{\text {shr: }}$ The lateral shower profile for electrons, defined as

$L_{\mathrm{shr}}=0.14 \frac{\sum_{i}\left(M_{i}-P_{i}\right)}{\sqrt{\left(0.14 \sqrt{E_{e m}}\right)^{2}+\sum_{i}\left(\Delta P_{i}\right)^{2}}}$. Here the sums are over the towers in the $E M$ cluster adjacent to the seed tower and in the same wedge as the seed tower. $M_{i}$ and $P_{i}$ are the measured energy and predicted energy deposit in an adjacent tower.

- E: The electromagnetic cluster energy.

- $P$ : The COT momentum.

- $\Delta z$ : The distance in the r-z plane between the COT track and the matching CES cluster.

$-Q \cdot \Delta x$ : The distance in the $\mathrm{r}-\phi$ plane between the COT track and the matching CES cluster.

- $\chi_{\text {strip }}^{2}$ : A $\chi^{2}$ which comes from the comparison of the CES shower profile in the r-z plane with test beam data.

The selection cuts used are standard for many CDF analysis using central high $P_{T}$ electrons, and are given in Table 4.1 .

\subsubsection{Plug Electron Selection}

In addition to the central electrons, we also trigger on high $\eta$ electrons called 'plug electrons'. These plug electrons consist of a Plug Electromagnetic Calorimeter (PEM) and a Plug Electron Shower Maximum Detector (PES) calorimeter cluster matched to a silicon track using an outside-in tracking algorithm, called the Phoenix algorithm. This tracking algorithm identifies tracks using the position of the calorimeter cluster, the primary vertex position, and $E_{T}$ of the calorimeter cluster. The information from the primary vertex and calorimeter is combined to create tracks, which can then be used to seed a tracking algorithm similar to the outside-in tracking algorithm used for jet identification (Section 4.2.4). As with the central electrons, a number of variables are used to help identify plug electrons. Descriptions of these variables are as follows: 


\begin{tabular}{|l|}
\hline Selection Cut \\
\hline Fiducial \\
$E_{\mathrm{T}} \geq 20.0 \mathrm{GeV}$ \\
$\left|z_{0}\right| \leq 60.0 \mathrm{~cm}$ \\
$p_{\mathrm{T}}^{\text {tr }} \geq 10.0 \mathrm{GeV}$ \\
Good COT Axial Segments $\geq 3$ \\
Good COT Stereo Segments $\geq 2$ \\
$E_{\text {had }} / E_{\text {em }} \leq 0.055+0.00045 E$ \\
Isolation $\leq 0.1$ \\
$L_{\text {shr }} \leq 0.2$ \\
$E / P \leq 2.0$ if $P_{\mathrm{T}}<50.0 \mathrm{GeV} / \mathrm{C}$ \\
$|\Delta z| \leq 3.0 \mathrm{~cm}$ \\
$-3.0 \leq Q \cdot \Delta x \leq 1.5(\mathrm{~cm})$ \\
$\chi_{\text {strip }}^{2} \leq 10.0$ \\
\hline
\end{tabular}

Table 4.1: The selection cuts for central (CEM) electrons.

- $E_{\mathrm{T}}$ : The electromagnetic energy of the matching calorimeter cluster found using the Phoenix algorithm multiplied by the sine of the polar angle of the associated track.

- $\eta_{\mathrm{PES}}$ : The detector $\eta$ of the best matching PES cluster.

- Tower fit: Number of towers used by the PEM cluster fit.

- $E_{\text {had }} / E_{\text {em }}<0.05:$ The ratio of the hadronic calorimeter energy to the electromagnetic calorimeter energy.

$-\chi_{P E M}^{2}: \chi^{2}$ of the PEM cluster fit.

- Shower profile: The shower profile measures the ratio of the energy in the central five strips of the PES to the ratio of the energy in all nine strips.

- PHX match: A boolen variable indicating if the Phoenix track matches with the associated PES cluster.

- $\left|z_{0}\right|$ : Intersection of the track associated with the event with the beamline in the r-z plane.

- $N_{\text {Hits }}^{\mathrm{Si}}$ : The number of hits in the SVX detector that belong to the PHX track.

- $\Delta R(\mathrm{PES}, \mathrm{PEM}):$ The Distance in the $\eta-\phi$ plane between the plug electromagnetic calorimeter cluster and the best matching pre-shower cluster.

- Isolation: The ratio of the isolation energy in a cone of $\Delta \mathrm{R}=0.4$ to the energy of the matching EM object.

The selection cuts used are standard for many CDF analysis using forward high $P_{T}$ electrons, and are given in Table 4.2. 


\begin{tabular}{|l|}
\hline Selection Cut \\
\hline Plug electron \\
$E_{\mathrm{T}} \geq 20.0 \mathrm{GeV}$ \\
\hline $1.2<\eta_{\mathrm{PES}}<2.0$ \\
Tower fit \\
$E_{\mathrm{had}} / E_{\mathrm{em}}<0.05$ \\
$\chi_{P E M}^{2}<10.0$ \\
Shower profile \\
$\mathrm{PHX}$ match \\
$\left|z_{0}\right| \leq 60.0 \mathrm{~cm}$ \\
$N_{\text {Hits }}^{\mathrm{Si}} \geq 3$ \\
$\Delta R(\mathrm{PES}, \mathrm{PEM}) \leq 3.0$ \\
Isolation $\leq 0.1$ \\
\hline
\end{tabular}

Table 4.2: The selection cuts for plug (PHX) electrons. 


\subsubsection{Muon Selection}

Muon candidates consist of a high $P_{T}$ COT track that extrapolates to a track segment (stub) in one of the muon chambers, either CMU and CMP or CMX. These candidates are identified using a set of variables, described as follows:

$-p_{\mathrm{T}}$ : The transverse momentum of associated COT track.

- $p^{\text {corr }}$ : The momentum of associated COT track.

- $E_{\text {had }}$ and $E_{\text {em }}$ : The energy deposited in the electromagnetic and hadronic calorimeters, respectively. For muon candidates, these should be consistent with the energy expectation of a minimum ionizing particle.

- $\left|z_{0}\right|$ : The intersection of the track and beamline in the r-z plane.

- CMU/CMP/CMX $|\Delta x|$ : The distance in the $r-\phi$ plane between the stub direction and the COT track extrapolated to the muon chamber.

- CMU/CMP/CMX Fiducial: A check that the track extrapolated from the tracking chamber to the plane of the muon chamber is in the fiducial region of the detector used.

- The number of good axial and stereo COT segments.

- $\left|d_{0}\right|:$ If the track has silicon hits, this is measured with respect to the SVX beam spot. Otherwise, this is measured with respect to the COT beamspot.

- Isolation: The ratio of the total energy in a cone of $\Delta R=0.4$ to the transverse momentum of the muon.

The selection cuts used are standard for many CDF analysis using high $P_{T}$ muons, and are given in Table 4.3. 


\begin{tabular}{|l|}
\hline Selection Cut \\
\hline$p_{\mathrm{T}}^{\text {corr }} \geq 20.0 \mathrm{GeV} / \mathrm{c}$ \\
\hline$E_{\text {had }} \leq \max \left(6,6+0.028\left(p^{\text {corr }}-100\right)\right)$ \\
$E_{\mathrm{em}} \leq \max \left(2,2+0.0115\left(p^{\text {corr }}-100\right)\right)$ \\
$\left|z_{0}\right| \leq 60.0 \mathrm{~cm}$ \\
\hline for CMUP muons only: \\
CMU $|\Delta x| \leq 7.0 \mathrm{~cm}$ \\
CMP $|\Delta x| \leq 5.0 \mathrm{~cm}$ \\
CMU fiducial \\
CMP fiducial \\
\hline for CMX muons only: \\
CMX $|\Delta x| \leq 6.0 \mathrm{~cm}$ \\
CMX fiducial \\
\hline Good COT Axial Segments $\geq 3$ \\
Good COT Stereo Segments $\geq 2$ \\
$\left|d_{0}\right| \leq 0.2 \mathrm{~cm}$ if no Si hits \\
$\left|d_{0}\right| \leq 0.02 \mathrm{~cm}$ if Si hits \\
\hline Isolation $\leq 0.1$ \\
\hline
\end{tabular}

Table 4.3: The baseline cuts for CMUP and CMX muons. The cosmic veto is only applied to data, not Monte Carlo events. 


\subsubsection{Jet Selection}

Our event selection uses an algorithm with conesize of $\Delta R=0.4$ to reconstruct the jets. This is an iterative algorithm that builds the largest energy cone with radius 0.4 around a given seed cluster in the calorimeter. All energy within a size 0.4 cone is associated with the jet.

\subsubsection{Jet Energy Corrections}

The reconstructed jets are corrected for several effects. These corrections take the form of eight possible jet correction levels.

Jet correction level 0 sets up the calorimeter energy scale. This includes setting the Central Electromagnetic Calorimeter (CEM) scale to the $Z \rightarrow e e$ peak, and the Central Hadronic Calorimeter (CHA) scale to the peak expected of minimum ionizing particles. The Wall Hadron Calorimeter (WHA) scale is tracked with source calibrations and the PEM/PHA (Plug Electromagnetic Calorimeter/Plug Hadronic Calorimeter) and photomultiplier tube (PMT) scales are also tracked with calibration results.

Jet energy correction level 1 is also referred to as the "eta-dependent" or "relative correction". This correction adjusts raw jet energies measured in the calorimeter to make jet response uniform along $\eta$.

Jet energy correction levels 2 and 3 are no longer used. The numbering scheme is kept for historical reasons.

Jet energy correction level 4 is referred to as the "multiple interactions" correction. At this level, jets are corrected for energy from different $p \bar{p}$ interactions occurring during the same bunch crossing, which would increase the energy of the measured jet. The correction removes the average energy contribution from multiple events, as derived from minimum bias data. It is parameterized as a function of the number of $p \bar{p}$ interaction verticies in the event.

Jet energy correction level 5 is the "absolute" energy correction. At this level jets are corrected for any non-linearity and energy loss in un-instrumented regions of the calorimeters. This is the jet correction level applied for our single top event selection.

For the level 6 jet energy correction, the jet is corrected for energy associated with the spectator partons in the hard collision (or the "underlying event").

The final jet correction level used is the level 7 or "out of cone" correction. This corrects for particle-level energy leakage of radiation outside the clustering cone used for jet definition. In essence this takes the jet energy back to the parent parton energy. This correction level is applied to jets used in kinematic variables for the single top search. 


\subsubsection{B Jet Identification}

B-tagging, or identifying b-quark jets, is critical for the identification of top quarks. Fortunately, the b-quark has a unique signature that enables us to more easily identify b-quark jets. These features include the long lifetime of the B hadrons, their large mass compared to other hadrons, and their energetic semileptonic decay.

There are several $b$-jet identification techniques used at CDF. The single top analysis uses a technique called secondary vertex tagging (SECVTX tagging) [5], which is reliant on the long lifetime of $\mathrm{B}$ hadrons. The b-quark travels about $3 \mathrm{~mm}(\beta \gamma c \tau)$ before decaying, creating a decay vertex called the 'secondary vertex'. The secondary vertex is distinct from the original $p \bar{p}$ interaction point, or 'primary vertex'. The impact parameter resolution of the silicon detectors is about $40 \mu \mathrm{m}$, allowing reconstruction of both the primary and secondary verticies of the event. SECVTX tagging uses information from the primary and secondary verticies to identify b-quark jets. Variables used include:

$-d_{o}$ : The impact parameter.

- $L_{x y}$ : The 2D decay length, or distance between the primary and secondary verticies in the event.

- $S_{d_{o}}: d_{o}$ significance, $S_{d_{o}}=\left|\frac{d_{o}}{\delta_{d_{o}}}\right|$

- $S_{L_{x y}}$ : The decay length significance, $S_{L_{x y}}=\left|\frac{L_{x y}}{\delta_{L_{x y}}}\right|$

Some of these quantities are illustrated in Figure 4.1.

The SECVTX algorithm begins by considering silicon tracks associated with each jet. These tracks must be seeded or confirmed by a track in the COT, and must have $p_{T}>0.5 \mathrm{GeV} / \mathrm{c}, S_{d_{o}}>2.0$, a minimum number of hits in the silicon tracking detectors and finally the tracks must not exceed a maximum $d_{o}$ requirement. A secondary vertex is then sought among these tracks. If a 'seed' vertex is found, the remaining tracks are considered for vertexing, and after attaching all qualifying tracks, a vertex $\chi^{2}$ is calculated and tracks are iteratively pruned if they contribute too much to the $\chi^{2}$.

At this point, a number of cuts are applied to the candidate secondary vertex. First, there must be three or more tracks associated with the vertex. Then further cuts are applied which include the removal of material and nuclear interactions and interactions consistent with $K_{s}$ and $\Lambda$ decay, as well as a cut on $S_{L_{x y}}>7.5$.

If no candidate secondary vertex passes all the cuts, a second pass is made. In this case candidate secondary verticies must have two or more tracks, and must pass a set of cuts more stringent than the first pass candidates. Such candidates are called second pass candidates.

Most b-tags are reconstructed in the positive direction. In this case, the secondary vertex is in the same side of the detector as the jet. There are also b- 


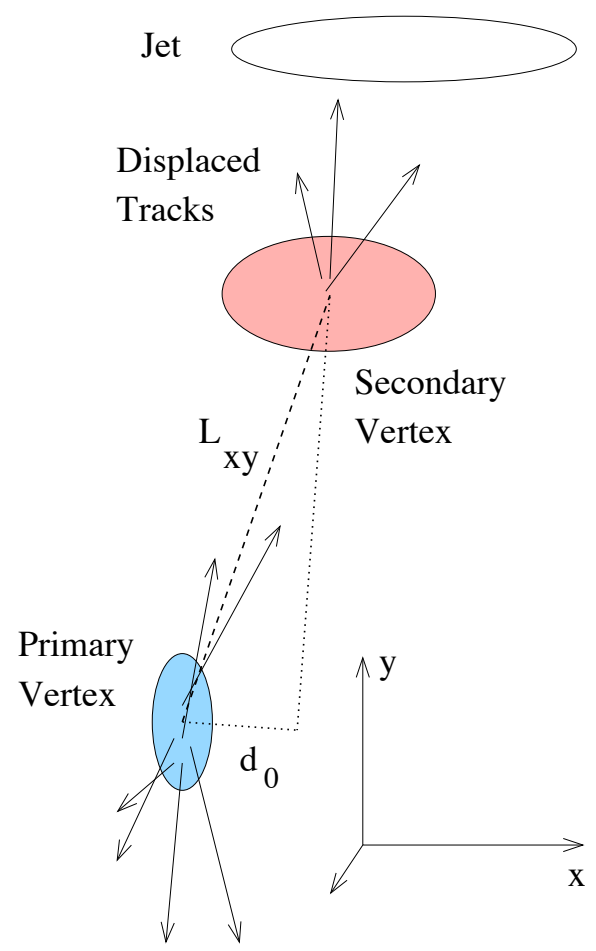

Prompt tracks

Figure 4.1: Primary and Secondary verticies with associated track quality quantities. 
tags that are reconstructed in the negative direction where the secondary vertex is on the opposite side of the detector as the jet (behind the jet). These are considered to be mistags, since no tags naturally occur with a negative decay length in our high $P_{T}$ sample.

\subsubsection{Neutrino Reconstruction}

Evidence for a neutrino in the CDF detector is detected in the form of missing transverse energy $\left(\mathbb{E}_{T}\right) . \mathbb{E}_{T}$ is defined as the vector sum of the transverse energy summed over all calorimeter towers.

$$
E_{T}=-\sum_{i} E_{T}^{i} \hat{n}_{i}
$$

Here, the sum over $i$ is a sum over all the calorimeter towers with $\eta<3.6, \hat{n}_{i}$ is a unit vector perpendicular to the beam axis pointing to the $i$ th calorimeter tower, and $\sum_{i} E_{T}^{i}=E_{i} \sin \theta_{i}$ where $E_{i}$ is the energy deposited in the $i$ th calorimeter towner and $\theta_{i}$ is the polar angle of the tower. This transverse energy is corrected for the vertex position. A correction is also applied for the transverse momenta of muons, applied by adding all the transverse momenta of all muons to the sum and removing the average energy deposited in the calorimeter. This is necessary since muons pass through the calorimeters as minimum ionizing particles. A final correction is applied to account for the difference between raw jets and the level 5 jet corrections used in the event selection. In this case, the $E_{T}$ is corrected for the difference between the transverse energy of level 0 and level 5 corrected jets. In applying the jet correction for this case, the number of primary verticies is set to unity. This prevents the energy from additional proton-antiproton interactions from being subtracted in the jet corrections to $\mathbb{E}_{T}$.

The missing transverse energy does not give enough information to fully reconstruct the neutrino, leaving some ambiguity in event reconstruction. The $z$-component of the neutrino momentum is unknown, but can be reconstructed in various ways. One simple neutrino reconstruction algorithm takes advantage of the assumption that the neutrino and lepton are formed by a real $W$ boson. In this case, the neutrino momentum can be calculated up to a two-fold ambiguity using the kinematic constraint that the lepton and neutrino are consistent with a coming from $W$ boson, as follows:

$$
\begin{array}{r}
p_{w}=p_{l}+p_{\nu} \\
m_{W}=\sqrt{\left(p_{l}+p_{\nu}\right)^{2}}
\end{array}
$$

Solving this quadratic equation for $\mathrm{z}$ component of $p_{\nu}$ we find that: 


$$
\begin{array}{r}
p_{z}(\nu)=\frac{-b \pm \sqrt{\left(b^{2}-4 a c\right)}}{2 a} \\
a=p_{x}^{2}(l)+p_{y}^{2}(l) \\
b=-\left(m_{w}^{2} p_{z}(l)+2 p_{x}(l) p_{z}(l) E_{T}, x+2 p_{y}(l) p_{z}(l) E_{T, x}\right) \\
c=E_{T, y}\left(p_{x}^{2}(l)+p_{z}^{2}(l)\right)+E_{T, x}\left(p_{y}^{2}(l)+p_{z}^{2}(l)\right) \\
-\frac{1}{4} m_{W}^{4}-m_{W}^{2} p_{x}(l) E_{T, x}-m_{W}^{2} p_{y}(l)=E_{T, y}-2 p_{x}(l) p_{y}(l) E_{T, x} E_{T, y} \\
m_{w}=80.45 \pm 0.04 G e V / c^{2}
\end{array}
$$

The smallest $\left|p_{z} \nu\right|$ solution is chosen and when the solution obtained is complex, the real portion of the $p_{z}(\nu)$ solution is taken, ie, $p_{z}(\nu)=\frac{-b}{2 a}$. This choice is correct approximately $77 \%$ of the time in t-channel single top Monte Carlo. For any given $p_{z}(\nu)$ choice, the neutrino energy is taken to be $E(\nu)=\sqrt{\left(E_{T}^{2}+p_{z}(\nu)^{2}\right)}$. This simple neutrino reconstruction is for some kinematic variables which are not highly dependent on the top quark mass. For other variables, a more complex reconstruction is preformed using a kinematic solver technique. In this case, information about the jets and missing energy is combined to find a b-jet and $p_{z}(\nu)$ solution most consistent with the top mass and $W$ mass hypotheses. This procedure will be described in more detail in Section 6.1.1

\subsection{Event Selection Cuts}

The single top event selection consists of a series of cuts designed to optimize the signal to background ratio of the data. First, a cut is made on the primary vertex position of the event $|z|<60 \mathrm{~cm}$. This is to ensure that the event is well measured with the CDF detector. Since the single top signature consists of a lepton, neutrino, and two or three jet with at least one b-quark jet, the single top sample is required to contain all these objects. The event is required to have exactly one high $P_{T}$ lepton, either a central or plug lepton or a muon. This lepton must pass the selection cuts as outlined, and there must be no additional high $P_{T}$ leptons in the event. All electrons must have $|\eta|<1.6$. Additionally, this lepton must be within $5 \mathrm{~cm}$ of the primary vertex of the event. Two or three jets are required with $\eta<2.8$ and $E_{T}>20 \mathrm{GeV}$ using a level 5 jet energy correction. At least one of these jets is required to have a secondary vertex b-tag. Finally, it is required that the $E_{T}$, corrected using level 5 jets, be greater than $25 \mathrm{GeV}$.

After this initial selection, a series of event vetoes are utilized. This includes a veto on $Z$-bosons, a dilepton veto, a conversion veto and a cosmic ray veto. Finally, we require a special QCD cut designed to separate QCD background from our signal sample, since this background is poorly understood kinematically. Details on these event vetoes are explained below. 


\subsubsection{Background Vetoes}

For single top selection, the event is expected to contain exactly one lepton. Thus, a strict dilepton veto is applied. Exactly one CMUP, CMX, PHX or CEM lepton is expected, so events with any additional leptons are vetoed. This includes 'tight' leptons, well defined leptons measured in the CMUP, CMX, PHX, CEM and BMU detectors, as well as looser lepton types consisting of muons which would not meet $\mathrm{CDF}$ trigger requirements. This veto helps to reduce the amount of $t \bar{t}$ production events in our event sample.

In addition to the dilepton veto, we apply an even stricter dilepton veto in the $Z$-peak region. The $Z$ boson veto is essentially a cut on the mass peak of the $Z$-boson, between 75 and $105 \mathrm{GeV}$. All events are removed in which the identified lepton and a loosely identified lepton track form an invariant mass in this region. This is designed to remove Z-events that might otherwise mimic the $\mathrm{W}+$ jets signature of single top events.

Conversions, or photons which convert to $e^{+} e^{-}$pairs in the tracking chambers, are a background which is easy to control via event selection. For our purposes, a conversion is defined as a pair of oppositely charged tracks, one of which is identified as a lepton, with small separation in the $r-\phi$ plane $(<2 \mathrm{~mm})$ and small difference in the polar angle. To control this background, we veto all events with identified conversion pairs. If a third track also satisfies the conversion definition, however, no veto is applied. Such events are interpreted as a 'trident' electron or an electron which emits a high energy bremsstrahlung photon which then converts into an electron/positron pair.

Another background which is easy to control via event selection is cosmic rays. A tight timing cut is applied around the Tevatron collision time, selecting only events consistent with the beam crossing.

\subsubsection{QCD Veto}

A veto is also applied to remove much of the QCD or "nonW" background. Since this background is difficult to model, it is desirable to cut as many QCD events as possible from the final sample. This is accomplished by a series of cuts on the reconstructed $W$ Boson and $E_{T}$.

Variables used in this veto include:

- $\left|E_{T}\right|$ : The magnitude of $E_{T}$.

- $M_{T}(W)$ : The transverse mass of the reconstructed $W$ Boson. $M_{T}(W)=\sqrt{\left(P_{T}(l e p) \times\left|E_{T}\right|-P_{x}(l e p) \times\left|E_{T}\right|_{x}-P_{y}(l e p) \times\left|E_{T}\right|_{y}\right.}$.

- $\Delta \phi(l, j e t):$ The angle between the lepton and the reconstructed jet.

- $\Delta \phi\left(E_{T}, j e t\right):$ The angle between the $E_{T}$ and the reconstructed jet.

- $E_{T}$ (sig): $E_{T}$ significance is defined as the magnitude $E_{T}$ divided by the uncertainty in that quantity, $\frac{\underline{z}_{T}}{\sigma_{\mathbb{F}_{T}}}$. 


\begin{tabular}{|c|c|c|}
\hline & 2 -jet & 3 -jet \\
\hline CEM & $M_{T}(W)>20 \mathrm{GeV}$ & $M_{T}(W)>20 \mathrm{GeV}$ \\
& $E_{T}($ sig $) \geq-0.05 \times M_{T}(W)+3.5$ & $E_{T}($ sig $) \geq-0.05 \times M_{T}(W)+3.5$ \\
& $E_{T}($ sig $) \geq-7.6+3.2 \times \Delta \phi(l, j e t 1)$ & $E_{T}($ sig $) \geq-2.5+(2.5 / 0.8) \times \Delta \phi\left(E_{T}\right.$, jet 1$)$ \\
\hline PHX & $M_{T}(W)>20 \mathrm{GeV}$ & $M_{T}(W)>20 \mathrm{GeV}$ \\
& $E_{T}($ sig $)>2$ & $E_{T}($ sig $)>2$ \\
& $E_{T}>45-30 \times \Delta \phi\left(E_{T}\right.$, jet 1$)$ & $E_{T}>45-30 \times \Delta \phi\left(E_{T}\right.$, jet 1$)$ \\
& & $E_{T}>45-30 \times \Delta \phi\left(E_{T}\right.$, jet 2$)$ \\
\hline CMUP & $M_{T}(W)>10 \mathrm{GeV}$ & $M_{T}(W)>10 \mathrm{GeV}$ \\
& $E_{T} \geq-145+60 \times \Delta \phi(l, j e t 1)$ & $M_{T}(W)>10 \mathrm{GeV}$ \\
\hline CMX & $M_{T}(W)>10 \mathrm{GeV}$ & \\
\hline
\end{tabular}

Table 4.4: QCD veto for CEM, PHX, CMUP and CMX lepton types. The veto is given for both the 2 and 3 jet bins.

\begin{tabular}{|l|c|c|}
\hline lepton type & t-channel & s-channel \\
\hline CEM & $94 \%$ & $96 \%$ \\
PHX & $86 \%$ & $87 \%$ \\
CMUP & $97 \%$ & $98 \%$ \\
CMX & $97 \%$ & $98 \%$ \\
\hline
\end{tabular}

Table 4.5: QCD efficiencies for CEM, PHX, CMUP and CMX lepton types. Efficiencies are the fractions of single top events surviving the QCD removal cut, measured using either t-channel or s-channel Monte Carlo.

A unique set of cuts is applied for a given number of jets and lepton type (either CEM, PHX, CMUP or CMX). Table 4.4 gives a summary of the cuts applied.

Since QCD modeling is somewhat poorly understood, the QCD veto is optimized using data and background W+Jets Monte Carlo events. All selection cuts were applied except the b-tagging requirement in order to obtain a sample with similar features to the final data sample, but larger statistics. It is assumed that regions with a large amount of data and small amount of Monte Carlo contained a large amount of QCD background, and cuts were placed on such regions.

While the QCD veto removes a significant portion of the non-W background, high signal efficiencies are retained, as shown in Table 4.5. 


\section{Signal and Background Modeling}

For the multivariate likelihood analysis, a complete model of all signal and background processes is needed, as well as an accurate estimate of the signal and background components of the data. The signal and background models are needed to model kinematic distributions used as inputs to the multivariate likelihood function. The final result is highly sensitive to kinematic mis-modelings, so care must be taken in estimating the model for each signal or background process. This is done using a variety of Monte Carlo and data samples, which will be discussed in the sections below. The background model used is similar to Reference [5].

\subsection{Monte Carlo Event Generation}

In this analysis, two main types of Monte Carlo were used, matrix element generators and parton shower generators. Matrix element generators, such as "MadEvent" [72] and "Alpgen" [73], calculate leading order matrix elements. Matrix element generators calculate observables in the parton picture, but can be paired with a parton shower generator for modeling of hadronization, a process in which the partons are converted to real observable hadrons with the help of phenomenological models. Parton shower generators use leading order matrix elements for the calculation of subprocesses and then allow these subprocesses to radiate additional partons ("parton showering") and undergo hadronization.

\subsubsection{Parton Shower Generators}

Parton shower generators are used to generate several important pieces of the single top background model. The generator Pythia is used to model $t \bar{t}$ and diboson production, as well as the parton showering and hadronization of several backgrounds calculated using matrix element generators. Pythia is a general purpose parton shower generator with an emphasis on multi-parton production in elementary particle collisions. Both initial and final state showers are ordered using $Q^{2}$, the momentum transfer or virtuality. Beam remnants and multiple interactions are taken into account. Finally, the fragmentation and decay of the particles is calculated using the Lund string model [71]. 


\title{
5.1.2 Matrix Element Generators
}

Matrix element generators used in this analysis include MadEvent, used to model single top production, and Alpgen, used to model $W$ and $Z$ boson production in association with one or more jets.

\begin{abstract}
Alpgen
The Alpgen [73] event generator is specially designed to generate multiparton hard process events. It is based on leading order calculations of parton amplitudes. Similar amplitudes are computed together to make the phase space integration more efficient. For instance, instead of summing over all flavor configurations, a single flavor independent matrix element is multiplied by appropriate parton densities or CKM factors. For each event, one subprocess is calculated with all subprocesses selected with uniform probability. The accumulated rates of each subprocess are then used to weight the event selection probabilities in subsequent integration iterations.

In order to combine Alpgen with a parton shower generator, however, unweighted events are needed. These can be generated via a two-step process. First, weighted events are generated as described above. The seed of the random number used to generate the event and the event weight is stored to a file. Then, the generator code is run a second time, recalculating the events in the file using the stored seed and unweighting each event using the maximum weight of sample and the weight of each event. Finally, a color flow is assigned to the event [73].
\end{abstract}

\section{MadEvent}

MadEvent is an event generator based on MadGraph [72]. For any given process, MadGraph identifies all relevant subprocesses, generates their amplitudes, and produces mappings for integration over phase space. MadEvent then produces a stand alone code that can be used to calculate cross sections and produce unweighted events.

The MadEvent generator relies on a Feynman diagram expansion for the phase space integration. The efficiency of this algorithm is increased by reweighting channels by their contributions to the total result, and by grouping similar diagrams together [72].

The MadGraph code is also used in a stand alone fashion elsewhere in the analysis. The matrix element amplitudes calculated by MadGraph are used as a kinematic variable in the multivariate likelihood search. This is described in more detail in Section 6.1.1. 


\begin{tabular}{|l|c|c|}
\hline Sample Name & Type & Generator Used \\
\hline stop00 & $s$-channel & MadEvent + Pythia \\
stopm0 & $t$-channel LO/NLO matched & MadEvent + Pythia \\
\hline
\end{tabular}

Table 5.1: Monte Carlo Samples used in the single top model. The sample name, signal type modeled, and Monte Carlo generator used are given for each sample. The sample name is a CDF internal designation given to each sample, listed here for completeness.

\subsection{Detector Simulation}

The response of the CDF Run II detector is modeled using a detailed simulation based on the GEANT3 package [74]. This simulation has several components, including a models for the silicon chambers, a drift model for the COT simulation [75],[76], and a calorimeter simulation [77]. A detailed description can be found in Reference [78].

\subsection{Single Top Modeling}

\subsubsection{Single Top Monte Carlo}

The single-top Monte Carlo used in this analysis was made using MadEvent with Pythia parton showering. The $s$-channel Monte Carlo consisted of a simple LO Feynman diagram, as shown in Figure 5.1, diagram c. The modeling of $t$ channel single-top production, however, is more difficult [37]. The kinematic distributions are not well predicted by a using only the leading order diagram compared to the full NLO prediction. The $p_{T}$ distribution of the second $b$ jet is too soft, and the $\eta$ distribution of this $b$ jet is too forward. Thus, a mix of leading order and next to leading order Monte Carlo must be used.

Two diagrams are used, also shown in Figure 5.1, diagrams a and b. The leading order diagram used is the $2 \rightarrow 2$ process, $b+q \rightarrow q^{\prime}+t$ and the nextto-leading-order $2 \rightarrow 3$ process, $\bar{b}+g+q \rightarrow q^{\prime}+t+\bar{b}$. By including one of the most important next-to-leading-order diagrams, the modeling of kinematic distributions is much more accurate. The $2 \rightarrow 2$ and $2 \rightarrow 3$ processes need to be mixed together. This is done by adjusting the ratio between the two processes to reproduce the $P_{T}$ distribution of the second $b$ quark jet predicted by full next-to-leading-order Monte Carlo. For this purpose, a mix of the two MadEvent diagrams was compared to the ZTOP [37][38] prediction, made at full next-to-leading-order. The matching procedure is described in more detail elsewhere [79]. The final matched Monte Carlo was compared to the ZTOP predictions. Kinematic output distributions of several quantities were compared, and a systematic uncertainty was calculated on the acceptance. More details can be found in Reference [80]. A listing of the signal Monte Carlo samples used is given in Table 5.1. 
a)

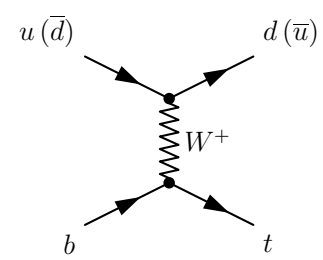

b)

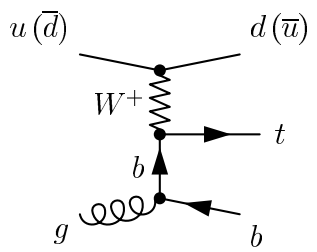

c)

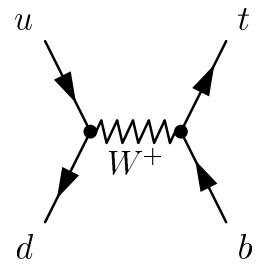

Figure 5.1: Feynman diagrams for single-top production. a) $t$-channel $2 \rightarrow 2$ process, assumes the introduction of a $b$ quark PDF; b) $t$-channel $2 \rightarrow 3 \mathrm{~W}$ gluon-fusion diagram, which is an NLO contribution if a $b$ quark PDF is used; c) LO $s$-channel $\left(W^{*}\right)$ diagram.

\subsubsection{Event Detection Efficiencies}

The first step in the single-top analysis is a determination of the number of signal and background events we expect in our data sample. The single top analysis cuts 4.3 are applied to the MadEvent Monte Carlo to determine the fraction of events that pass the selection requirements, $\epsilon_{\mathrm{evt}}^{\mathrm{MC}}$. This fraction is combined with a number of scale factors and efficiencies to determine the total number of signal events expected, $N_{\text {signal }}$.

$$
N_{\text {signal }}^{\text {predict }}=\sigma_{\text {st }}^{\text {theo }} \cdot \epsilon_{\text {evt }} \cdot \mathcal{L}_{\text {int }}
$$

Here, $\epsilon_{\text {evt }}$ can be decomposed into several factors:

$$
\epsilon_{\mathrm{evt}}=\epsilon_{\mathrm{evt}}^{M C} \cdot B R \cdot S F_{z 0} \cdot \epsilon_{\mathrm{trig}} \cdot S F_{\mathrm{reco} / \mathrm{ID}} \cdot \epsilon_{\text {tag }}
$$

Here, $\epsilon_{t a g}$ is a term calculated from the b-tag scale factor using a combinatoric scheme detailed below in Equations 5.3 and 5.4. In this equation, $\epsilon_{\mathrm{evt}}^{\mathrm{MC}}$ is simply the ratio of the total number of events which pass all selection cuts (see Section 4.3) in the Monte Carlo to the total number of events generated.

In the single top Monte Carlo sample, the $W$ boson was only allowed to decay into leptons: $W \rightarrow e / \mu / \tau+\nu$. This has to be taken into account by applying the factor $B R=0.324$ [21]. This is the well known branching ratio for the $W$ boson to decay into leptons. (Processes such as $W \rightarrow c \bar{s}, c \rightarrow l \nu X$ are not included.)

The $z$ vertex cut efficiency, measured in data, has the value of $\epsilon_{z 0}^{\mathrm{data}}=0.964 \pm$ 0.005. This is combined with the efficiency of this cut in Monte Carlo to give the scale factor, $S F_{z 0}=\frac{\epsilon_{z 0}^{\text {data }}}{\epsilon_{z 0}^{M C}}$, which has a value of 1.029 .

The trigger efficiency, $\epsilon_{\text {trig }}$, is given in Table 5.2. This efficiency is calculated separately for the four lepton types: CEM, PHX, CMUP, and CMX. For central electrons, the efficiency is composed of a tracking and calorimeter efficiency. The tracking efficiency is studied by comparing the number of events which pass a trigger with tight calorimeter requirements and no tracking requirements to 
the number of events which also pass the CEM trigger requirement, both using the $W \rightarrow e \nu$ selection. The calorimeter trigger efficiency is calculated at L1 using events collected by muon triggers and at L2 and L3 by a trigger path that requires the L1 trigger to have fired. The trigger is prescaled at L2 and has very loose cuts at L3. The PHX trigger efficiency is similarly calculated by comparing events in similar trigger paths, using $W \rightarrow e \nu$ or $Z \rightarrow e^{+} e^{-}$events. For PHX events, in addition to the flat trigger efficiency, each event is weighted by a trigger turn-on factor, parameterized as a function of missing- $E_{T}$ and $E_{T}$ of the electron. This correction was found to be needed by comparing distributions in data and the Monte Carlo events without tagging for PHX electrons. The correction helps account for kinematic differences between triggered data and the Monte Carlo backgrounds. Finally, the muon trigger efficiencies are calculated using $Z \rightarrow \mu^{+} \mu^{-}$events, comparing the number of events where both muons are triggered to the number where only one muon is triggered.

The lepton ID scale factors are calculated from $Z \rightarrow e^{+} e^{-}$and $Z \rightarrow \mu^{+} \mu^{-}$ events. One of the leptons is required to pass tight reconstruction requirements in all cases. In data, this lepton must also be matched to a lepton capable of firing the trigger. A second lepton is then sought, the stub lepton, which passes loose requirements. For muons, the loose muon is simply an additional track. The track plus tight muon has an invariant mass within the $Z \rightarrow \mu^{+} \mu^{-}$window, and the probe passes if it is matched to a muon stub. For electrons, the probe is an extremely loose electromagnetic calorimeter cluster which forms an invariant mass with the tight electron that is within the $Z \rightarrow e^{+} e^{-}$window. Finally, the probe is required to meet tight electron requirements, listed in Section 4. For both the muon and electron cases, the scale factor is computed from the data/Monte Carlo ratio. For electrons, the value of $S F_{\text {reco/ID }}$ is simply given by this ratio while for muons an additional reconstruction scale factor is applied.

The muon reconstruction scale factor is also calculated using $Z \rightarrow \mu^{+} \mu^{-}$ events. Here, it is required that one muon is found passing tight muon requirements in both data and Monte Carlo. In data, this tight muon must also be matched to a muon capable of firing the muon trigger. A $Z$ peak is reconstructed by finding the invariant mass of the muon plus an additional track. The muon stub efficiency is then found in the data and Monte Carlo by checking if the second track is matched to a muon stub. The scale factor is computed from the data/Monte Carlo ratio. For the muon case, the lepton and reconstruction scale factors are combined together in $S F_{\text {reco/ID }}$. These values are given in Table 5.2.

\begin{tabular}{|c|c|c|}
\hline & Trigger Efficiency & ID Scale Factor/Reconstruction Scale Factor \\
\hline CEM & $0.965 \pm 0.0044$ & $0.978 \pm 0.0050$ \\
PHX & $0.946 \pm 0.0027$ & $0.932 \pm 0.007$ \\
CMUP & $0.915 \pm 0.0054$ & $0.926 \pm 0.0067$ \\
CMX & $0.937 \pm 0.0076$ & $0.970 \pm 0.0097$ \\
\hline
\end{tabular}

Table 5.2: Electron and muon trigger efficiencies, and ID efficiency scale/ Reconstruction scale (correction) factors. 
The $b$ tag scale factor, $S F_{t a g}$ is calculated using two methods. The electron method takes advantage of the fact that jets which contains electrons ("electron jets") are enriched in heavy flavor due to semileptonic decays, while electron jets that also contain a conversion partner form a complementary sample with fewer heavy flavor jets. An efficiency is calculated by selecting electron jets and comparing the tag rate of jets in which this electron is found to be part of a conversion pair to the tag rate of non-conversion electron jets, in both data and Monte Carlo. The scale factor is then calculated by taking the ratio of the data and Monte Carlo efficiencies. For the muon method, jets containing a muon are selected and a simulation is used to provide templates for $p_{T}(\mathrm{rel})$, the transverse momentum of the muon relative to the jet for $b$ and non- $b$ jets. Fits of this distribution in data and Monte Carlo are then used to determine the fraction of tagged and non-tagged $b$ jets and to calculate the tagging efficiency for such jets. The scale factor is calculated by the ratio of this efficiency in data and Monte Carlo. For the final $b$ tag scale factor, the results of the two methods are combined. The final value of the scale factor is calculated as $S F_{t a g}=0.95 \pm 0.01$.

In order to calculate the tagging efficiency, $\epsilon_{\text {tag }}$ each Monte Carlo event is weighted by the probability of that event being tagged. This is calculated using Equation 5.3. Here $p_{t a g}=S F_{t a g}$ for jets with a SECVTX tag, and 0 for heavy flavor jets with no SECVTX tag.

$$
P_{\text {event }}^{\text {tag }}=1-\Pi_{i}^{\text {jets }}\left(1-p_{\text {tag }}^{i}\right)
$$

The tagging efficiency is then calculated using Equation 5.4. Here, the number of pretag events is defined as the number of events which pass all selection cuts except the SECVTX tag requirement.

$$
\epsilon_{\text {tag }}=\frac{\sum_{j}^{e v e n t s} P j^{\text {tag }}}{N_{\text {pretag }}}
$$

The value for $\epsilon_{\text {evt }}$ can be used to calculate the number of expected events according to (5.1), these results are given in Section 5.5.2. 


\subsection{Background Modeling}

\subsubsection{The Mistag Matrix and Mistag Asymmetry Corrections}

\section{The Mistag Matrix}

There are a variety of reasons that the SECVTX algorithm could cause mistags: false positive tags that come from an incorrect identification of a secondary vertex in a jet which doesn't contain a heavy-flavor quark. Tracks in light quark jets could have spuriously large impact parameters because of limited detector resolution, long lived light-particle decays $\left(\Lambda, K_{s}\right)$, or material interactions. For many of these mistags, the rate of positively and negatively tagged jets are the same. Here, a positively tagged jet has a 2D decay length greater than zero where the secondary interaction vertex is consistent with the direction of the tracks in the jets. A negatively tagged jet has a 2D decay length less than zero, where the secondary interaction is on the opposite side of the detector behind the jet. To a good approximation a secondary vertex from a mistag is equally likely to have a positive or negative $2 \mathrm{D}$ decay length, and most vertices with negative decay lengths are spurious. Thus, the number of negatively tagged jets is a good estimate of the light-flavor contribution to the positive sample, although corrections are applied to account for differences between the tagging rates for positively and negatively tagged jets. Diagrams of a typical mistag and well real $b$ jet are shown in Figure 5.2.

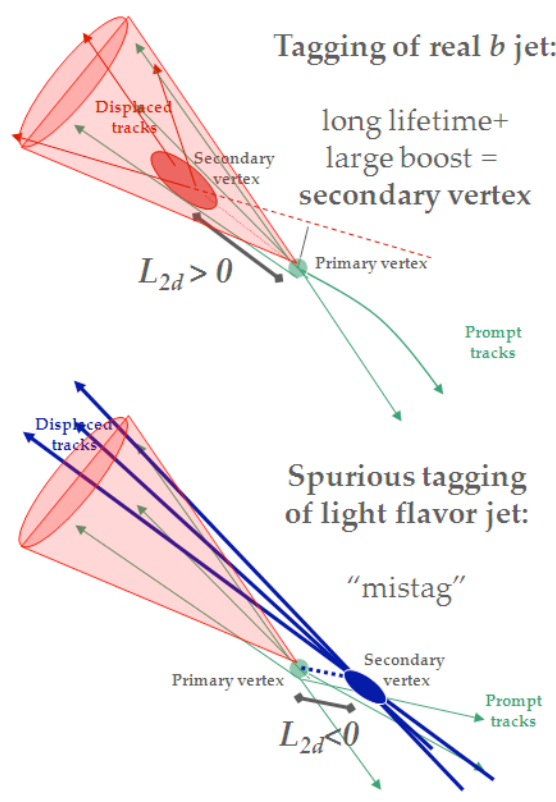

Figure 5.2: Figures demonstrating a typical negatively tagged mistag jet and a real, positively tagged, $b$ jet. 
However, simply counting the number of negative tags in our W+Jets Monte Carlo yields a sample with very low statistics. Instead, a parametrization of the negatively tagged jets is made using a generic jet sample. The generic jet sample consists of a set of jet triggers completely distinct from the single top data. The properties of the negative tags in these data samples are parametrized in six variables: $E_{T}(\mathrm{GeV})$, and $|\eta|$ of the jet, the track multiplicity $\left(N_{t r k}\right)$, the sum of the transverse energies of all the taggable jets in the event $\left(\sum E_{T}\right)$, the number of primary interactions in the event (\# $\mathrm{Z}$ vertices), and the $\mathrm{z}$ position of the primary vertex of the event (PZV). For this purpose, a jet is considered taggable if $E_{T}>10 \mathrm{Gev}$ and $|\eta|<2.4$ and the jet contains at least two reconstructed tracks. Each jet is compared against this parametrization, and the probability for a given jet to be mistagged is taken to be the normalized contents of the parameterization bin in which that event falls. A full explanation of the tagging matrix, including a derivation of the systematics, can be found in Appendix A.

The mistag matrix used was created using only $1.2 \mathrm{fb}^{-1}$ of data. In order to account for any differences between this smaller dataset and the full $2.2 \mathrm{fb}^{-1}$ dataset used in the analysis, an additional scale factor was applied to the negative tag rate, inflating the rate slightly. The scale factor was derived by comparing predicted and observed tagging rates for inclusive jet data using the full $2.2 \mathrm{fb}^{-1}$ dataset. The predicted and observed tag rates were compared for variables used to parametrize the matrix $\left(\mathrm{E}_{T}(\mathrm{GeV}), \mathrm{N}_{t r k}, \sum E_{T},|\eta|\right.$, \# Z vertices, $\mathrm{PZV}$ ), and this was used to derive a scale factor of $S F_{M M}=1.06 \pm 0.05$.

\section{Mistag Matrix Asymmetry Corrections}

Although for many of the sources of mistag jets, such as detector resolution, the rates are the same for positively and and negatively tagged jets, some sources, such as contributions from long-lived light particles and material interactions, result in only in jets with positive secondary vertices. An adjustment must be made to the mistag probability to account for these differences. The asymmetry factor, $\gamma$, was evaluated by fitting the Monte Carlo distribution of the pseudo-

lifetime $c \tau=L_{x y} \times \frac{M_{v t x}}{p_{T_{v t x}}}$ to data. The asymmetry factor, given as a function of $E_{T}$ of the jet, is shown in Table 5.3. For each jet, the complete mistag rate is then taken to be the mistag probability calculated by the mistag matrix multiplied by the appropriate asymmetry correction.

\begin{tabular}{|c|c|c|c|c|}
\hline & $10-22 \mathrm{GeV}$ & $22-40 \mathrm{GeV}$ & $40-60 \mathrm{GeV}$ & $60-200 \mathrm{GeV}$ \\
\hline$\gamma$ & $1.36 \pm 0.20$ & $1.30 \pm 0.14$ & $1.44 \pm 0.12$ & $1.54 \pm 0.13$ \\
\hline
\end{tabular}

Table 5.3: Mistag asymmetry factor, derived from a fit of the pseudo-lifetime.

\subsection{2 $\mathrm{W}+$ Jets and $\mathrm{Z}+$ jets}

The $\mathrm{W}+$ Jets background is modeled using Alpgen Monte Carlo with Pythia parton showering. Samples used can roughly be divided into three categories: 
$\mathrm{W}+$ jets, $\mathrm{W}+c+$ jets, and $\mathrm{W}+\mathrm{QQ}+$ jets (where $\mathrm{QQ}$ is either $b \bar{b}$ or $c \bar{c}$ ). Here, the $W$ boson decays leptonically into a lepton and neutrino (all three lepton types are considered). The events are divided into $0,1,2,3$ and $4+$ jets categories. A full listing of the samples used is given in Table 5.5. The events are weighted according to the cross section of the production process.

The samples resulting from this procedure produce events which overlap in phase space, since parton showering can cause promotion of an $n$ parton event to an $n+1$ parton event. A matching procedure is applied to ensure that portions of phase space are simulated only once, and simulated by a matrix element whenever possible. This "MLM matching" scheme is described in detail elsewhere [81]. An additional jet-based heavy flavor overlap removal scheme is also applied.

The $\mathrm{W}+$ Jets sample is then split into $W+b \bar{b}, W+c / W+c \bar{c}(\mathrm{~W}+\mathrm{HF})$ and $\mathrm{W}+$ Light Flavor $(\mathrm{W}+\mathrm{LF})$ samples. First, each jet is identified as either a $b$, $c$ or light quark jet. A $b$-jet is defined as any jet matched to a generator jet containing a bottom hadron with non-zero $p_{T}$. A $c$-jet is any non- $b$-jet matched to a generator jet containing a charm hadron. All other jets are defined as light flavor jets. Events with at least one $b$-jet are classified as $W+b \bar{b}$, events with at least one $c$-jet and no $b$-jet are classified as $W+c / W+c \bar{c}$, and other events are classified as $\mathrm{W}+\mathrm{LF}$. However, we remove $\mathrm{W}+\mathrm{LF}$ events which were originally generated as $W+b \bar{b}$ or $W+c \bar{c} / W+c$ events because they contaminate the light flavor jets with tracks from heavy flavor decays and distort the tag predictions. These correspond to events produced as $\mathrm{W}+\mathrm{HF}$ where none of the identified jets is matched to $\mathrm{a} b$ hadron, either because the $\mathrm{b}$ hadron is lost or has zero $p_{T}$.

In the $\mathrm{W}+\mathrm{LF}$ case, the taggable jets are assigned to be 'tagged'. If there are two taggable jets in the events, the event is used twice with each jet being tagged for one event. The value for the neural network $b$ tagger output is randomly assigned to the jet attributed as tagged based a template obtained from simulated light flavor jets.

Finally, the events are weighted by a factor incorporating the scale factor and mistag rate associated with each jet in the event. Each jet is assigned an individual weight. If the jet is identified as a $b$ or $c$ jet as described above, the weight is the b-tag scale factor if the jet is SECVTX tagged, and zero otherwise. If the jet is identified as a light jet, the weight is given by the weight calculated using the mistag matrix and asymmetry correction. Weights for all the jets in an event are combined to obtain a total b-tag/mistag weight for the event. This is then multiplied by the cross section weight to obtain the total event weight.

The $\mathrm{Z}+$ jets events are produced in a similar fashion, using matched Alpgen samples with Pythia showering. The samples generated are listed in Tables 5.4 and 5.5 . 


\begin{tabular}{|l|c|l|}
\hline Sample Name & Type & Generator Used \\
\hline Z+Jets & & \\
ztopp0 & $\mathrm{Z}(e e)+0 \mathrm{p}$ & Alpgen + Pythia \\
ztopp1 & $\mathrm{Z}(e e)+1 \mathrm{p}$ & Alpgen + Pythia \\
ztop2p & $\mathrm{Z}(e e)+2 \mathrm{p}$ & Alpgen + Pythia \\
ztop3p & $\mathrm{Z}(e e)+3 \mathrm{p}$ & Alpgen + Pythia \\
ztop4p & $\mathrm{Z}(e e)+\geq 4 \mathrm{p}$ & Alpgen + Pythia \\
ztopp5 & $\mathrm{Z}(\mu \mu)+0 \mathrm{p}$ & Alpgen + Pythia \\
ztopp6 & $\mathrm{Z}(\mu \mu)+1 \mathrm{p}$ & Alpgen + Pythia \\
ztop7p & $\mathrm{Z}(\mu \mu)+2 \mathrm{p}$ & Alpgen + Pythia \\
ztop8p & $\mathrm{Z}(\mu \mu)+3 \mathrm{p}$ & Alpgen + Pythia \\
ztop9p & $\mathrm{Z}(\mu \mu)+\geq 4 \mathrm{p}$ & Alpgen + Pythia \\
ztopt3 & $\mathrm{Z}(\tau \tau)+0 \mathrm{p}$ & Alpgen + Pythia \\
ztopt4 & $\mathrm{Z}(\tau \tau)+1 \mathrm{p}$ & Alpgen + Pythia \\
ztopt2 & $\mathrm{Z}(\tau \tau)+\geq 2 \mathrm{p}$ & Alpgen + Pythia \\
ztopb0 & $\mathrm{Z}(e e)+\mathrm{bb}+0 \mathrm{p}$ & Alpgen + Pythia \\
ztopb1 & $\mathrm{Z}(e e)+\mathrm{bb}+1 \mathrm{p}$ & Alpgen + Pythia \\
ztopb2 & $\mathrm{Z}(e e)+\mathrm{bb}+\geq 2 \mathrm{p}$ & Alpgen + Pythia \\
ztopb5 & $\mathrm{Z}(\mu \mu)+\mathrm{bb}+0 \mathrm{p}$ & Alpgen + Pythia \\
ztopb6 & $\mathrm{Z}(\mu \mu)+\mathrm{bb}+1 \mathrm{p}$ & Alpgen + Pythia \\
ztopb7 & $\mathrm{Z}(\mu \mu)+\mathrm{bb}+\geq 2 \mathrm{p}$ & Alpgen + Pythia \\
ztopbt & $\mathrm{Z}(\tau \tau)+\mathrm{bb}+\geq 0 \mathrm{p}$ & Alpgen + Pythia \\
ztopc0 & $\mathrm{Z}(e e)+\mathrm{cc}+0 \mathrm{p}$ & Alpgen + Pythia \\
ztopc1 & $\mathrm{Z}(e e)+\mathrm{cc}+1 \mathrm{p}$ & Alpgen + Pythia \\
ztopc2 & $\mathrm{Z}(e e)+\mathrm{cc}+\geq 2 \mathrm{p}$ & Alpgen + Pythia \\
ztopc5 & $\mathrm{Z}(\mu \mu)+\mathrm{cc}+0 \mathrm{p}$ & Alpgen + Pythia \\
ztopc6 & $\mathrm{Z}(\mu \mu)+\mathrm{cc}+1 \mathrm{p}$ & Alpgen + Pythia \\
ztopc7 & $\mathrm{Z}(\mu \mu)+\mathrm{cc}+\geq 2 \mathrm{p}$ & Alpgen + Pythia \\
ztopct & $(\tau \tau)+\mathrm{cc}+\geq 0 \mathrm{p}$ & Alpgen + Pythia \\
\hline
\end{tabular}

Table 5.4: Monte Carlo Samples used in the Z+Jets Model. The sample name, background type modeled, and Monte Carlo generator used are given for each sample. The sample name is a CDF internal designation given to each sample, listed here for completeness. 


\begin{tabular}{|c|c|c|}
\hline Sample Name & Type & Generator Used \\
\hline W+Jets and Z+Jets & $W(e \nu)+b b+0 \mathrm{p}$ & Alnoen + Pythia \\
\hline btop1w & $\mathrm{W}(e \nu)+\mathrm{bb}+1 \mathrm{p}$ & Alpgen + Pythia \\
\hline btop2w & $\mathrm{W}(e \nu)+\mathrm{bb}+\geq 2 \mathrm{p}$ & Alpgen + Pythia \\
\hline btop5w & $\mathrm{W}(\mu \nu)+\mathrm{bb}+0 \mathrm{p}$ & Alpgen + Pythia \\
\hline btop6w & $\mathrm{W}(\mu \nu)+\mathrm{bb}+1 \mathrm{p}$ & Alpgen + Pythia \\
\hline btop $7 w$ & $\mathrm{~W}(\mu \nu)+\mathrm{bb}+\geq 2 \mathrm{p}$ & Alpgen + Pythia \\
\hline dtop0w & $\mathrm{W}(\tau \nu)+\mathrm{bb}+0 \mathrm{p}$ & Alpgen + Pythia \\
\hline dtop1w & $\mathrm{W}(\tau \nu)+\mathrm{bb}+1 \mathrm{p}$ & Alpgen + Pythia \\
\hline dtop $2 \mathrm{w}$ & $\mathrm{W}(\tau \nu)+\mathrm{bb}+\geq 2 \mathrm{p}$ & Alpgen + Pythia \\
\hline ctop0w & $\mathrm{W}(e \nu)+\mathrm{cc}+0 \mathrm{p}$ & Alpgen + Pythia \\
\hline ctop1w & $\mathrm{W}(e \nu)+\mathrm{cc}+1 \mathrm{p}$ & Alpgen + Pythia \\
\hline ctop $2 \mathrm{w}$ & $\mathrm{W}(e \nu)+\mathrm{cc}+\geq 2 \mathrm{p}$ & Alpgen + Pythia \\
\hline ctop $5 \mathrm{w}$ & $\mathrm{W}(\mu \nu)+\mathrm{cc}+0 \mathrm{p}$ & Alpgen + Pythia \\
\hline ctop6w & $\mathrm{W}(\mu \nu)+\mathrm{cc}+1 \mathrm{p}$ & Alpgen + Pythia \\
\hline ctop $7 \mathrm{w}$ & $\mathrm{W}(\mu \nu)+\mathrm{cc}+\geq 2 \mathrm{p}$ & Alpgen + Pythia \\
\hline etopw0 & $\mathrm{W}(\tau \nu)+\mathrm{cc}+0 \mathrm{p}$ & Alpgen + Pythia \\
\hline etopw1 & $\mathrm{W}(\tau \nu)+\mathrm{cc}+1 \mathrm{p}$ & Alpgen + Pythia \\
\hline etopw2 & $\mathrm{W}(\tau \nu)+\mathrm{cc}+\geq 2 \mathrm{p}$ & Alpgen + Pythia \\
\hline stopw0 & $\mathrm{W}(e \nu)+\mathrm{c}+0 \overline{\mathrm{p}}$ & Alpgen + Pythia \\
\hline stopw1 & $\mathrm{W}(e \nu)+\mathrm{c}+1 \mathrm{p}$ & Alpgen + Pythia \\
\hline stopw2 & $\mathrm{W}(e \nu)+\mathrm{c}+2 \mathrm{p}$ & Alpgen + Pythia \\
\hline stopw3 & $\mathrm{W}(e \nu)+\mathrm{c}+\geq 3 \mathrm{p}$ & Alpgen + Pythia \\
\hline stopw5 & $\mathrm{W}(\mu \nu+\mathrm{c}+0 \mathrm{p}$ & Alpgen + Pythia \\
\hline stopw6 & $\mathrm{W}(\mu \nu+\mathrm{c}+1 \mathrm{p}$ & Alpgen + Pythia \\
\hline stopw7 & $\mathrm{W}(\mu \nu+\mathrm{c}+2 \mathrm{p}$ & Alpgen + Pythia \\
\hline stopw8 & $\mathrm{W}(\mu \nu+\mathrm{c}+\geq 0 \mathrm{p}$ & Alpgen + Pythia \\
\hline stopwa & $\mathrm{W}(\tau \nu)+\mathrm{c}+0 \mathrm{p}$ & Alpgen + Pythia \\
\hline stopwb & $\mathrm{W}(\tau \nu)+\mathrm{c}+1 \mathrm{p}$ & Alpgen + Pythia \\
\hline stopwc & $\mathrm{W}(\tau \nu)+\mathrm{c}+2 \mathrm{p}$ & Alpgen + Pythia \\
\hline stopwd & $\mathrm{W}(\tau \nu)+\mathrm{c}+\geq 0 \mathrm{p}$ & Alpgen + Pythia \\
\hline ptopw0 & $\mathrm{W}(e \nu)+0 \mathrm{p}$ & Alpgen + Pythia \\
\hline ptop1w & $\mathrm{W}(e \nu)+1 \mathrm{p}$ & Alpgen + Pythia \\
\hline ptop $2 w$ & $\mathrm{~W}(e \nu)+2 \mathrm{p}$ & Alpgen + Pythia \\
\hline ptop3w & $W(e \nu)+3 p$ & Alpgen + Pythia \\
\hline ptop $4 w$ & $\mathrm{~W}(e \nu)+\geq 4 \mathrm{p}$ & Alpgen + Pythia \\
\hline ptopw5 & $\mathrm{W}(\mu \nu)+0 \mathrm{p}$ & Alpgen + Pythia \\
\hline ptop6w & $\mathrm{W}(\mu \nu)+1 \mathrm{p}$ & Alpgen + Pythia \\
\hline ptop $7 w$ & $\mathrm{~W}(\mu \nu)+2 \mathrm{p}$ & Alpgen + Pythia \\
\hline ptop $8 w$ & $\mathrm{~W}(\mu \nu)+3 \mathrm{p}$ & Alpgen + Pythia \\
\hline ptop $9 w$ & $\mathrm{~W}(\mu \nu)+\geq 4 \mathrm{p}$ & Alpgen + Pythia \\
\hline utopw0 & $\mathrm{W}(\tau \nu)+0 \mathrm{p}$ & Alpgen + Pythia \\
\hline utop1w & $\mathrm{W}(\tau \nu)+1 \mathrm{p}$ & Alpgen + Pythia \\
\hline utop $2 w$ & $\mathrm{~W}(\tau \nu)+2 \mathrm{p}$ & Alpgen + Pythia \\
\hline utop3w & $\mathrm{W}(\tau \nu)+3 \mathrm{p}$ & Alpgen + Pythia \\
\hline utop $4 w$ & $\mathrm{~W}(\tau \nu)+>4 \mathrm{p}$ & Alpgen + Pythia \\
\hline
\end{tabular}

Table 5.5: Monte Carlo Samples used in the W+Jets Model. The sample name, background type modeled, and Monte Carlo generator used are given for each sample. The sample name is a CDF internal designation given to each sample, listed here for completeness. 


\subsection{3 $t \bar{t}$ and Diboson}

The $t \bar{t}$ and diboson events are simulated using Pythia. Three types of diboson events were generated, including WW, WZ and ZZ. A full list of the samples used can be found in Table 5.6.

\begin{tabular}{|l|c|c|}
\hline Sample Name & Type & Generator Used \\
\hline$t \bar{t}$, and Diboson & & \\
ttop75 & $t \bar{t}$ & Pythia \\
itopww & WW & Pythia \\
itopwz & WZ & Pythia \\
itopzz & Z & Pythia \\
\hline
\end{tabular}

Table 5.6: Monte Carlo Samples used for the $t \bar{t}$ and diboson samples. The sample name, background type modeled, and Monte Carlo generator used are given for each sample. The sample name is a CDF internal designation given to each sample, listed here for completeness.

\subsubsection{QCD}

Two models are used for the modeling of the QCD background in the single top search, the 'jet electron' model and the 'antielectron' model.

The jet electron model is based on the idea that one way for a QCD event to pass the selection cuts is to have a jet that fakes an electron. This model is based on a generic jet trigger sample, similar to the samples used to build the mistag matrix. A jet is selected with a set of cuts that resemble the lepton cut requirements. Specifically, the jet is required to have $E_{T}>20 \mathrm{Gev}, 0.05<$ $E_{\text {had }} / E_{\text {em }}<0.02$, and the event is required to contain at least four jets.

Once a jet is identified as a jet electron, it is removed from the jet counting and from the $E_{T}$ correction. Jet electrons are treated as electrons and the event is subjected to the normal single top selection cuts (4.3) to the pretag level. The SECVTX b-tag requirement is dropped to increase the statistics of the sample. Kinematic quantities for the jet electron are calculated from the jet identified as an electron, and the charge of the jet electron is assigned randomly. The jet electron sample has good coverage in $\eta$ and can be used to model all lepton types, including those in the forward regions.

The antielectron model is designed to find electron candidates which nearly pass the electron kinematic cuts. Such candidates are required to pass all kinematic cuts outlined in Section 4.2, but fail two of the five non-kinematic cuts, on CES $\Delta x$, CES $\Delta z, E_{\text {had }} / E_{\text {em }}$, or $L_{\text {shr. }}$. Such candidate events are QCD enriched, but still maintain many of the kinematic properties of $W$ events, including isolation. As with the jet electrons, antielections events are subjected to the standard selection cuts to the pretag level. Unlike the jet electron model, antielectrons can only be used to model leptons in the central region since the model is based only on central electron samples.

A combination of jet electron and antielectron models is used in the single top 
analysis. In the central regions, where both the antielectron and jet electron models are valid, we use a combination of the two models. The events are replicated for various muon and electron types (CEM, CMUP, CMX), with the appropriate QCD cuts applied. The jet electron sample is used for the high eta region, where the antielectron sample is not valid.

Since the pretag sample is used for modeling this background, it is necessary to derive an algorithm for which jet will be used as the 'tagged' jet. In this case, we consider the taggable jets in each event. If there is only one taggable jet in the event, it is taken to be the tagged jet. If there are two taggable jets, the event is duplicated and the first jet is assigned to be tagged for one event and the second jet for the other. We also duplicate the events with one taggable jet.

In addition to assigning the jet to be taken as tagged, a value needs to be assigned for the jet for the neural network b-tagger. For this case, the events are events used three times, varying the hypothesis of the tagged jet as b, c or light flavor. The type is then weighted by a fraction of the flavor measured by the neural net b-tagger: $45 \%$ b, $40 \%$ c and $15 \%$ light quarks.

\subsection{Total Signal and Background Predictions}

\subsubsection{Background Prediction}

The background prediction for this analysis is composed of several pieces, the Monte Carlo based background estimate, the pretag QCD fit, the W+Jets estimation and finally the tagged QCD fit. Each of these pieces is described in detail below.

The first piece is the Monte Carlo based background estimate of $t \bar{t}$, diboson and $\mathrm{Z}+$ Jets. This is calculated similarly to the single top estimate, i.e.

$$
N_{\text {MCbackground }}^{\text {predict }}=\sigma_{\text {MCbackground }}^{\text {theo }} \cdot \epsilon_{\text {evt }} \cdot \mathcal{L}_{\text {int }}
$$

The event efficiency $\epsilon_{\mathrm{evt}}$, is calculated in the same manner as the single top efficiency calculation 5.3.2, neglecting the branching ratio $\epsilon_{\mathrm{BR}}$ which is only applicable to the single top Monte Carlo. The cross sections used for these three processes are given in Table 5.7. For $t \bar{t}$, an additional dilepton scale factor is applied, as shown in Table 5.8. This is needed because the efficiency of the $t \bar{t}$ dilepton veto is different in data and Monte Carlo due to differences in lepton identification and reconstruction.

The second part of the background estimation consists of calculating the pretag QCD estimate. In this case, a sideband region of the data is considered where all selection cuts are applied except the SECVTX tagging cut and the $E_{T}$ requirement. An estimate of the non-QCD backgrounds is made, using the Monte Carlo based background estimate above and a $\mathrm{W}+$ Jets model obtained from weighted Alpgen events. The QCD and non-QCD backgrounds are fitted 


\begin{tabular}{|l|c|}
\hline Process & Cross Section in pb \\
\hline$t \bar{t}$ & $6.7 \pm 0.83$ \\
WW & $12.4 \pm 0.25$ \\
WZ & $3.96 \pm 0.06$ \\
ZZ & $1.58 \pm 0.05$ \\
Z+Jets & $787.4 \pm 50$ \\
\hline
\end{tabular}

Table 5.7: Cross sections for processes estimated using the Monte Carlo based background estimate. The $t \bar{t}$ cross section is taken from the Review of Particle Physics [21]. The WW,WZ and ZZ cross sections are calculated from the theoretical cross sections predicted for $\sqrt{s}=2.0 \mathrm{GeV}$ and $\sqrt{s}=1.8 \mathrm{GeV}$ calculated in Reference [82]. These cross sections are rescaled to $\sqrt{s}=1.96 \mathrm{GeV}$ using a linear and quadratic interpolation.

\begin{tabular}{|c|c|c|}
\hline 1 jet & 2 jet & 3 jet \\
\hline $1.079 \pm 0.02$ & $1.084 \pm 0.02$ & $1.079 \pm 0.02$ \\
\hline
\end{tabular}

Table 5.8: Dilepton Veto Scale Factor as a function of jet bin.

to the $E_{T}$ spectrum to obtain a QCD fraction. This fraction, $F_{Q C D}$ is then used to obtain the number of predicted pretag QCD events by simply multiplying this fraction by the total number of events in the signal region.

$$
N_{\mathrm{QCD}}^{\mathrm{pretag}}=F_{\mathrm{QCD}} \cdot N_{\mathrm{pretag}}
$$

For the central electron and muon regions, the antielectron sample is used for the QCD fits, and for the forward electron region the jet electron sample is used. The tagged QCD estimate is obtained by performing similar fits, where the entire background estimate including $\mathrm{W}+$ Jets is specified. In this case, the tagged antielectron and jet electron samples are used as described above.

The third step in the background estimate is the calculation of the $\mathrm{W}+\mathrm{HF}$ estimate. The number of pretag $\mathrm{W}+\mathrm{HF}$ events is given by the total number of pretag events with the Monte Carlo based backgrounds, single top signal, and QCD fraction subtracted out, i.e.:

$$
N_{\mathrm{W}+\mathrm{Jets}}^{\mathrm{pretag}}=N_{\text {pretag }} \cdot\left(1-F_{\mathrm{QCD}}\right)-N_{\mathrm{MCbkg}}-N_{\text {singletop }}
$$

To obtain the number of tagged $\mathrm{W}+\mathrm{HF}$ jets, the number of pretag HF jets is multiplied by correction factors, heavy flavor fractions, and the tagging efficiency as shown below.

$$
N_{\mathrm{W}+\mathrm{HF}}^{\mathrm{tag}}=N_{\mathrm{W}+\mathrm{HF}}^{\mathrm{pretag}} \cdot f_{\mathrm{HF}} \cdot K \cdot \epsilon_{\mathrm{tag}}
$$

Here $f_{\mathrm{HF}}$ is the fraction of events with jets matched to heavy flavor quarks, determined from Alpgen Monte Carlo. The tagging efficiency, $\epsilon_{t a g}$ is described in Section 5.3.2. The $K$ factor is a ratio of the fraction events with a $b$-jet in data compared with the fraction of events with a $b$-jet in Monte Carlo. The value $K=1.4 \pm 0.4$ was calculated in the 1 -jet bin. The fraction of events that are $b$ tagged was calculated by fitting the fraction of $b$-tagged jets in data from the 


\begin{tabular}{|c|c|c|c|c|}
\hline Process & 1-jet Prediction & 2-jet Prediction & 3-jet Prediction & 4-jet Prediction \\
\hline \hline$t$-channel & $17.1 \pm 2.5$ & $51.1 \pm 7.5$ & $14.9 \pm 2.2$ & $3.1 \pm 0.4$ \\
$s$-channel & $7.3 \pm 1.1$ & $33.4 \pm 4.8$ & $10.7 \pm 1.5$ & $2.4 \pm 0.3$ \\
$t \bar{t}(\ell+$ jets $)$ & $6.7 \pm 1.0$ & $74.7 \pm 10.7$ & $225.1 \pm 32.1$ & $270.2 \pm 38.3$ \\
$t \bar{t}$ dilepton & $5.6 \pm 0.8$ & $38.4 \pm 5.5$ & $31.6 \pm 4.5$ & $7.0 \pm 1.0$ \\
$W b \bar{b}$ & $569.0 \pm 172.4$ & $413.8 \pm 124.7$ & $120.5 \pm 36.4$ & $29.8 \pm 9.1$ \\
$W c c$ & $314.1 \pm 97.9$ & $184.6 \pm 56.9$ & $57.5 \pm 17.7$ & $15.9 \pm 4.9$ \\
$W c$ & $490.1 \pm 152.7$ & $159.9 \pm 49.3$ & $31.6 \pm 9.7$ & $6.1 \pm 1.9$ \\
$W+\mathrm{LF}$ & $769.9 \pm 100.5$ & $305.0 \pm 38.3$ & $88.5 \pm 11.3$ & $22.0 \pm 3.3$ \\
$Z+$ jets & $26.6 \pm 4.2$ & $19.3 \pm 2.9$ & $7.4 \pm 1.1$ & $2.0 \pm 0.3$ \\
$W W$ & $13.2 \pm 1.5$ & $36.0 \pm 4.0$ & $12.0 \pm 1.3$ & $3.1 \pm 0.3$ \\
$W Z$ & $6.6 \pm 0.5$ & $17.4 \pm 1.4$ & $4.8 \pm 0.4$ & $1.1 \pm 0.1$ \\
$Z Z$ & $0.1 \pm 0.0$ & $0.5 \pm 0.0$ & $0.2 \pm 0.0$ & $0.1 \pm 0.0$ \\
QCD & $43.0 \pm 17.2$ & $57.2 \pm 22.9$ & $21.0 \pm 8.4$ & $3.5 \pm 1.4$ \\
Total Prediction & $2269.5 \pm 434.3$ & $1391.2 \pm 235.9$ & $625.8 \pm 74.4$ & $366.3 \pm 42.3$ \\
Observed & $2472.0 \pm 0.0$ & $1394.0 \pm 0.0$ & $586.0 \pm 0.0$ & $409.0 \pm 0.0$ \\
\hline
\end{tabular}

Table 5.9: Method 2 Background Estimate

$W+1$ jet sideband region compared to Monte Carlo. The Monte Carlo used for this comparison was identical to that described in Section 5.4, and the data set used the same triggers described in Section 4.1, with an integrated luminosity of $1.5 \mathrm{fb}^{-1}$. A fit was made to both the SECVTX vertex mass distribution and the output of the Neural Network $b$ tagger. This fit is then used to compute the $K$ factor. The error is estimated by comparing the values obtained using the SECVTX fit and the Neural Network b tagger fit.

The calculation of the $\mathrm{W}+\mathrm{LF}$ estimate uses the mistag matrix described in Section 5.4.1. The number of spuriously tagged events, $N_{\text {mistag }}$ is calculated using the per-jet mistag rate and the correction factor $\gamma$, which is applied to correct for mistag asymmetry. The ratio of the spuriously tagged events to the total pretag events is then multiplied by a pretag $\mathrm{W}+\mathrm{LF}$ estimate, calculated by subtracting all the previously calculated backgrounds from the total number of pretag events $\left(N_{\text {pretag }}\right)$. The equation for the $\mathrm{W}+\mathrm{LF}$ calculation is given below:

$$
N_{\mathrm{W}+\mathrm{LF}}^{\mathrm{tag}}=N_{\text {mistag }} \cdot\left(1-F_{\mathrm{QCD}}^{\mathrm{pretag}}-\frac{N_{\mathrm{MCbkg}}-N_{\text {singletop }}-N_{\mathrm{HF}}}{N_{\text {pretag }}}\right)
$$

\subsubsection{Total Signal and Background Estimate}

The total signal and background estimate is given in Table 5.9. A plot of the resulting distribution of the number of jets is given in Figure 5.3. 


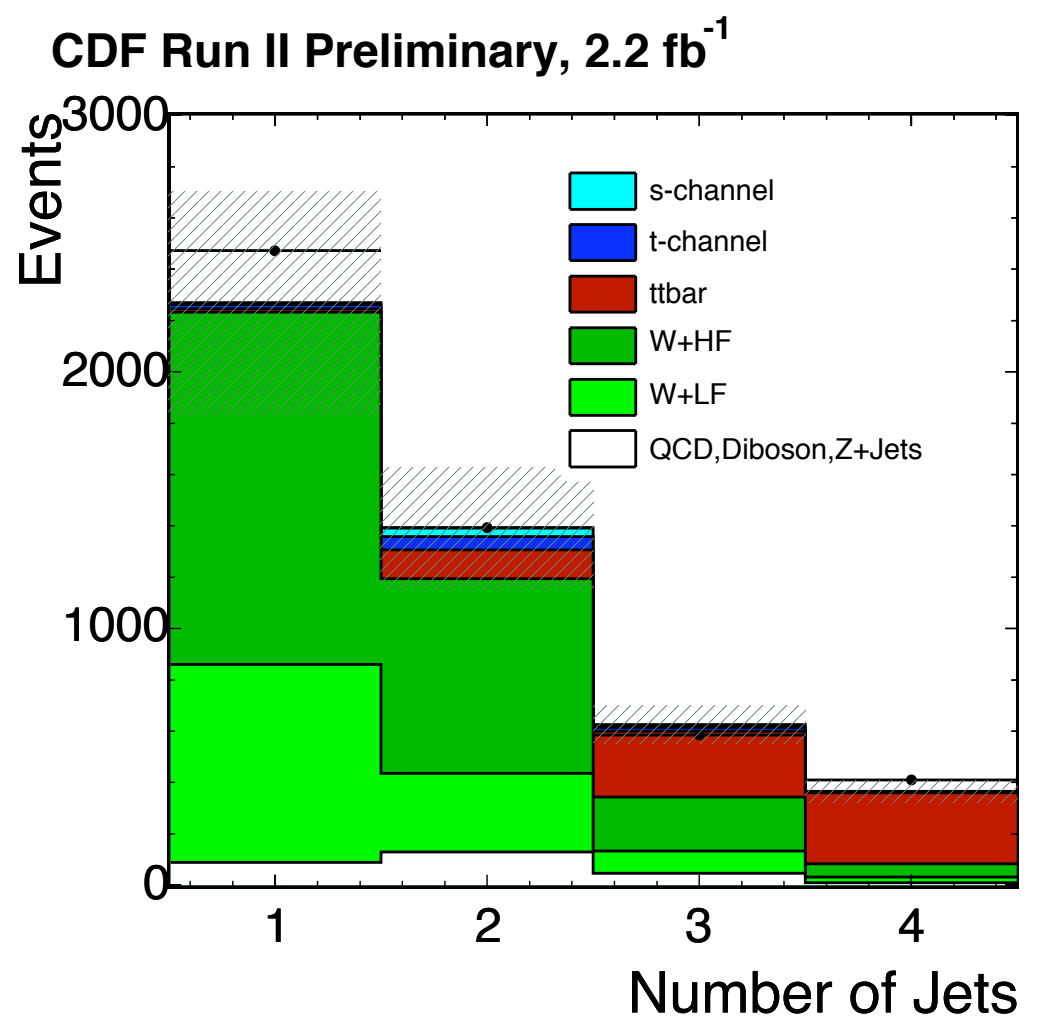

Figure 5.3: The background estimate as a function of the number of jets 


\section{Special Techniques}

\subsection{Kinematic Solver and Matrix Element Construction}

\subsubsection{Kinematic Solver}

In single top searches, there is significant ambiguity in calculating the reconstructed $M_{\ell \nu b}$. This ambiguity comes from uncertainty as to which jet to assign as the $b$ from top, uncertainty in the measurement of jet energies, and the fact that the $z$ component of missing energy is not measured. Some of the variables used in our multivariate analysis require knowledge of the top rest frame. Other variables, such as the MadGraph matrix element [72], are very sensitive to $M_{\ell \nu b}$. In either case, such variables are highly sensitive to the uncertainties mentioned above. The kinematic solver addresses these issues by finding the four-vectors of the $b$-jet and the neutrino which are the most consistent with measured values and have the top quark on shell.

Initially, the $P_{z}$ of the neutrino is calculated by constraining the mass of the lepton-neutrino system to the mass of the $W$ boson, taken to be $80.4 \mathrm{GeV} / \mathrm{c}^{2}$. This leads to a second-degree equation for $P_{z}(\nu)$, as explained in Section 4.2.7, with two possible solutions. Additionally, there are two choices of which jet is the $b$ from top decay in the 2-jet bin and three in the 3 -jet bin, giving a total of either 4 or 6 neutrino and $b$ jet solutions respectively. Only the momentum of the $b$ jet is used in the kinematic solver, with the remaining $b$ jet quantities always taking original pre-kinematic solver values. In contrast, all neutrino quantities are taken as inputs to the kinematic solver.

The kinematic solver takes each of the $b$-jet and neutrino pairs as described above and attempts to optimize them for consistency with the top mass of $175 \mathrm{GeV} / \mathrm{c}^{2}$ by pulling the jet energies and neutrino momentum until such consistency is achieved. A chi-squared function is then constructed quantifying how far the jet energies and neutrino momentum have been pulled from their original values and how consistent the final solution is with a top mass of 175 $\mathrm{GeV} / \mathrm{c}^{2}$. In the optimization step, the transverse momentum of the top quark, the $P_{T}$ of the $b$-jet and the mass of the $b$-jet are constrained to their original, prekinematic solver values. These constraints on the neutrino $b$-jet system allow the system to be solved for the $b$-jet energy ("corrected $b$-jet"). Here the $b$-jet mass is simply calculated from the energy and momentum quantities associated 

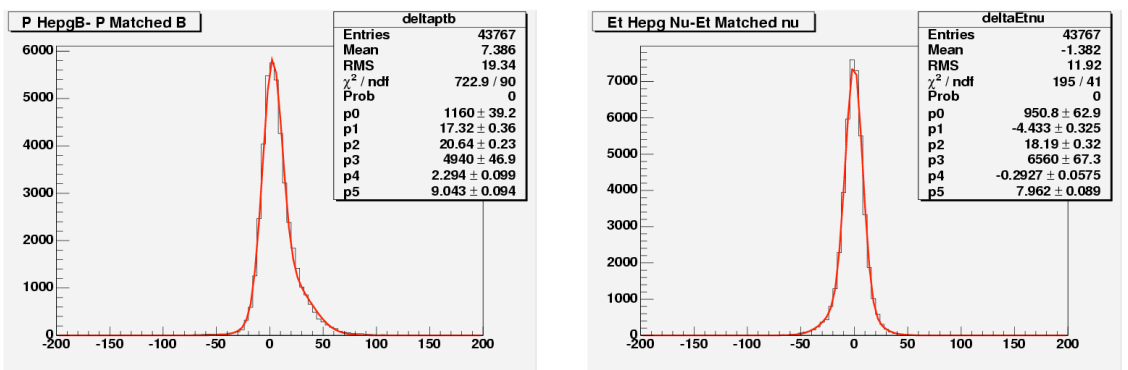

Figure 6.1: $\Delta P_{b}$, and $\Delta E_{T}$ in the 2-jet bin, with two Gaussian fits. These fits were used to calculate the values of $\sigma$ for the kinematic solver.

with the jet. A correction is then made to the $E_{T}$ for the difference between the original $b$-jet energy and the corrected $b$-jet, to retain the constraint on $M_{\ell \nu}$ of $80.4 \mathrm{GeV} / \mathrm{c}^{2}$. Finally, $M_{\ell \nu b}$ is computed and compared to $175 \mathrm{GeV} / \mathrm{c}^{2}$. If $M_{\ell \nu}$ can't be constrained to be real, a small amount of $E_{T}$ is added along the direction of the non-b jet, $\Delta \mathscr{E}_{T}$ until $M_{\ell \nu}$ is real. This procedure is iterated using Newton's Method.

A chi-squared function is then constructed as shown in Equation 6.1 below.

$$
\chi^{2}=\frac{\left(P_{b}-P_{b}^{o b s}\right)^{2}}{\sigma_{P_{b}}^{2}}+\frac{\left(\Delta E_{T}\right)^{2}}{\sigma_{\not_{T}}^{2}}+\frac{\left(M_{\ell \nu b}-M_{t}\right)^{2}}{\sigma_{M_{t}}^{2}}
$$

In Eq. 6.1, the following values are used in the 2-jet bin: $\sigma_{P_{b}}=9.04 \mathrm{GeV}$, and $\sigma_{\not \not_{T}}=7.96 \mathrm{GeV}$. In the 3-jet bin, the values are: $\sigma_{P_{b}}=8.75 \mathrm{GeV}$, and $\sigma_{\not F_{T}}=9.64 \mathrm{GeV}$. These values were derived by comparing fully reconstructed $t$-channel quantities to their corresponding values from the generator level signal Monte Carlo (Section 5.3). The distributions of $\Delta P_{b}$, and $\Delta \mathscr{E}_{T}$ were fit with two Gaussians, and the fit standard deviation of the narrower Gaussian was used as $\sigma$. These fits are shown in Figures 6.1 and 6.2. In addition, $\sigma_{M_{t}}=1 \mathrm{GeV}$ constrains the mass of the $\ell-\nu-b$ system to the top mass $M_{t}=175 \mathrm{GeV} / c^{2}$.

By construction, the top mass calculated using these solutions, $M_{\ell \nu b}^{k}$, is narrowly peaked around 175, as shown in Figure 6.3. Here, the reconstructed top mass is compared before and after the use of the kinematic solver. The output neutrino and $b$-jet four vectors can be used to reconstruct kinematic variables. The $\chi^{2}$ output from the kinematic solver, shown in Figure 6.4, can be used as a kinematic variable, and also is an input to the algorithm used to determine the choice of $b$-jet used in other kinematic variables. This algorithm is described further in Section 6.2. 

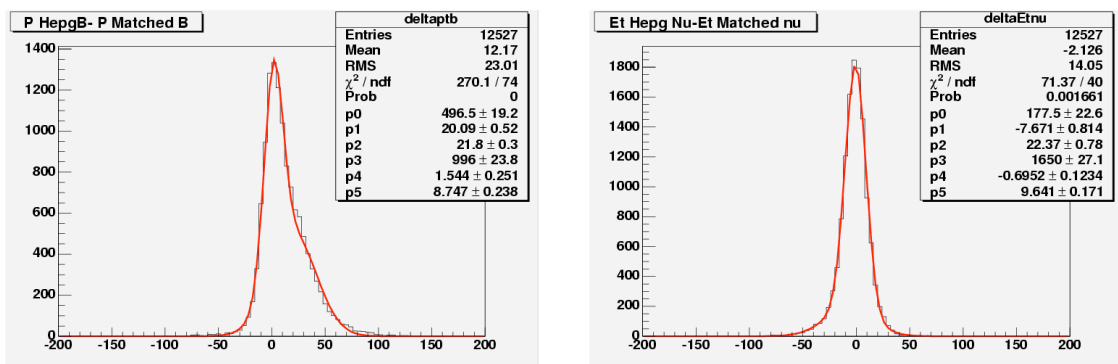

Figure 6.2: $\Delta P_{b}$, and $\Delta E_{T}$ in the 3-jet bin, with two Gaussian fits. These fits were used to calculate the values of $\sigma$ for the kinematic solver.
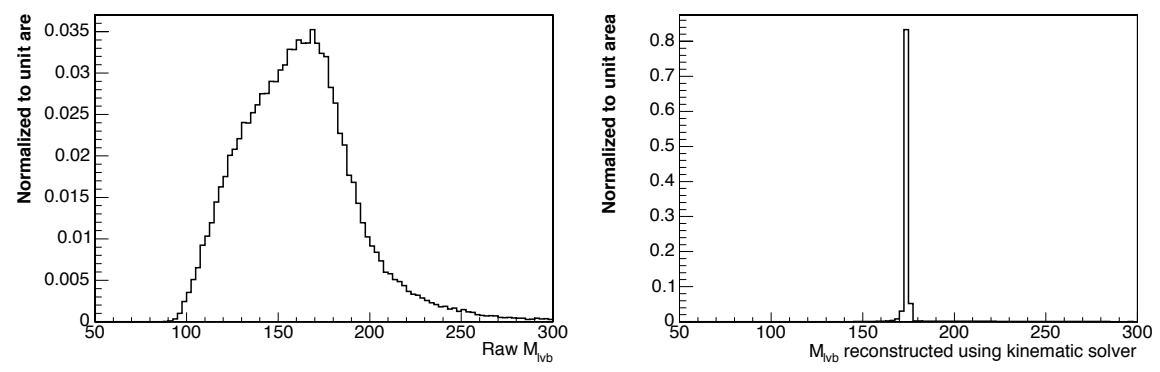

Figure 6.3: Raw $M_{\ell \nu b}$ and $M_{\ell \nu b}$ reconstructed with kinematic solver quantities. As expected, the effect of the kinematic solver is to cause the reconstructed top mass to have a narrow peak at 175 . Both plots are made using $t$-channel single top Monte Carlo. 


\section{CDF Run II Preliminary, $2.2 \mathrm{fb}^{-1}$}

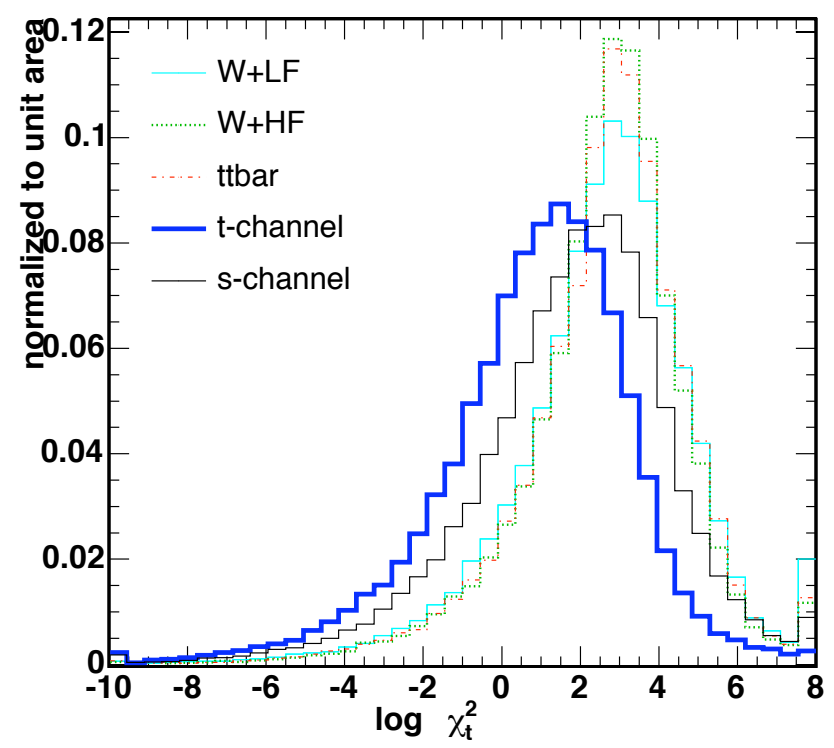

Figure 6.4: $\log$ of Kinematic solver $\chi^{2}$ template, plotted for $t$-channel and $s$-channel signal as well as $\mathrm{W}+\mathrm{HF}(W b \bar{b}, W c \bar{c}$ and $W c), \mathrm{W}+\mathrm{LF}$ and $t \bar{t}$ backgrounds.

\subsubsection{Matrix Element Calculation}

One set of variables which are calculated using kinematic solver quantities are the MadGraph [72] matrix elements. The MadGraph program allows a user to input initial and final state particles, and produce code to calculate helicity amplitudes for a given process. For the matrix element calculation, the initial state particles are taken to be massless $u$ and $\bar{d}$ quarks with initial energy and momentum split between the two quarks. It was found that this simple assumption led to better discriminating variables than a more complicated approach in which several initial states were combined. The momenta and energy of the final state particles were calculated using kinematic solver quantities for the $b$-jet, and $P_{z}(\nu)$. Using the kinematic solver is necessary since the MadGraph matrix elements require a top mass of $175 \mathrm{GeV} / \mathrm{c}^{2}$. Matrix elements for $t$-channel and $s$-channel signal top production were calculated, as well as the matrix element corresponding to $W b \bar{b}$ background. However, only the $t$-channel matrix element were chosen to be used in the final analysis as the other matrix elements did not add much separation between signal and background in the final likelihood function. Figure 6.5 shows the resulting $t$-channel Matrix Element distribution templates plotted for signal and background templates and a good discrimination is observed between signal and background. 
CDF Run II Preliminary, $2.2 \mathrm{fb}^{-1}$

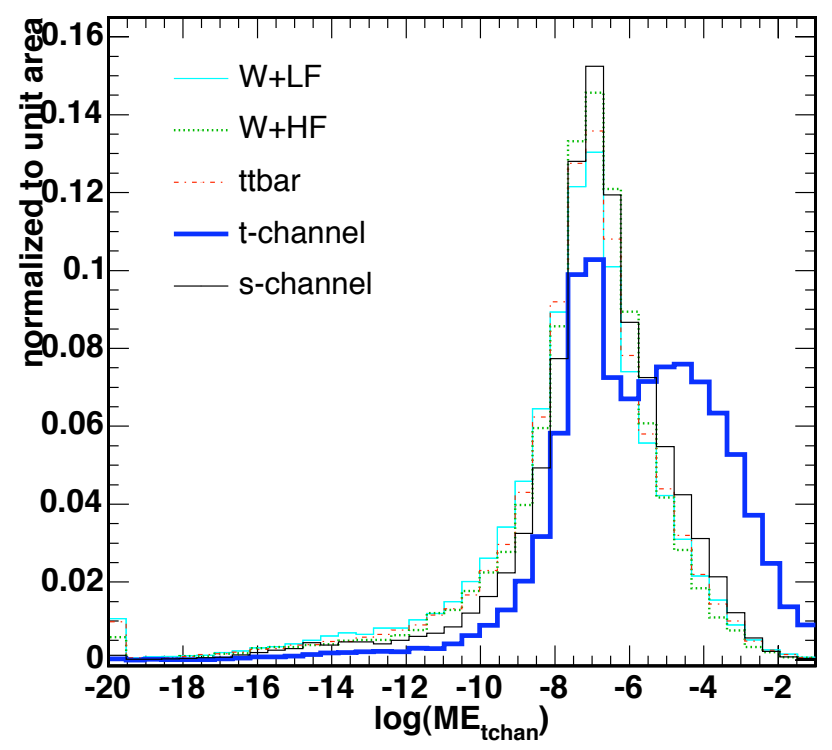

Figure 6.5: $t$-channel MADEVENT matrix element template, plotted for $t$ channel and $s$-channel signal as well as $\mathrm{W}+\mathrm{HF}(W b \bar{b}, W c \bar{c}$ and $W c), \mathrm{W}+\mathrm{LF}$ and $t \bar{t}$ backgrounds. 


\subsection{B-Choosing Algorithms}

In calculating kinematic variables for a single top search, one important decision is the choice of $b$ from top decay. For this purpose, $b$-choosing algorithms were devised using $t$-channel and $s$-channel Monte Carlo, see Chapter 5. In the 2-jet bin of the $t$-channel, this decision is relatively easy since $97 \%$ of events have exactly one tag as identified by the SECVTX algorithm, and of those tags nearly all of them can be matched to the generator level $b$ from top decay. This is due to the fact that although there are two $b$-quarks in a typical $t$-channel single top event, the $b$-quark not from the top decay is nearly always lost down the beamline. In both the 3 -jet bin and in the $s$-channel, b-choosing is more complicated. In the 3-jet bin of the $t$-channel, only $85 \%$ of the events have exactly one tag. In the $s$-channel, the 1 -tag rate is about $75 \%$, both in the 2 and 3 -jet bins. These results are summarized in Table 6.1

In the $s$-channel and, to a lesser degree, the $t$-channel 3-jet bin a large percentage of the jets that are closest in $\Delta \mathrm{R}$ ('matched') to the generator level $b$ from top decay are not tagged, as shown in Table 6.2. In these channels a successful b-choosing algorithm can not rely on SECVTX tagging alone. Fortunately, by combining b-tagging information with kinematic information from the event, the strength of the $b$-choosing algorithm can be greatly increased.

\begin{tabular}{|l|c|c|c|c|}
\hline Sample & $t$-chan (2-jet) & $s$-chan (2-jet) & $t$-chan (3-jet) & $s$-chan (3-jet) \\
\hline 1 tag & 97.28 & 76.75 & 86.04 & 74.91 \\
2 tags & 2.72 & 23.25 & 13.90 & 24.70 \\
3 tags & N/A & N/A & 0.06 & 0.39 \\
\hline
\end{tabular}

Table 6.1: Tag Multiplicity, in percent, for the 2 and 3 jet bins, using MADEVENT MC sample stopm0 for $t$-channel and stopo0 for $s$-channel,see chapter 5 .

\begin{tabular}{|l|c|c|c|c|}
\hline Sample & $t$-chan (2-jet) & $t$-chan (3-jet) & $s$-chan (2-jet) & $s$-chan (3-jet) \\
\hline all & 95.33 & 75.01 & 62.55 & 59.19 \\
1-tag bin & 95.20 & 71.21 & 51.20 & 47.58 \\
\hline
\end{tabular}

Table 6.2: Percentage of time the jet closest in $\Delta \mathrm{R}$ to the generator $b$ quark level jet is tagged. For events where the jet closest to the generator $b$ jet is not tagged, the tagging algorithm has failed to identify the actual $b$ jet.

Several possible input variables for the b-choosing algorithm were studied by looking at the power each variable showed distinguishing jets matched to the generator level $b$ quark in each jet channel. Strong variables included $Q_{\text {lep }} \times \eta$, the charge of the lepton times the $\eta$ of the jet candidate, the $\chi^{2}$ output from the kinematic solver, and $P_{T}$ of the jet. The choosing strength of these variables is given in Table 6.3.

Linear combinations of these variables were used to create a $b$-choosing algorithm. Weights for these variables were optimized using a fitting procedure. 


\begin{tabular}{|l|c|c|c|c|}
\hline Sample & $t$-chan(2-jet) & $t$-chan(3-jet) & $s$-chan(2-jet) & $s$-chan(3-jet) \\
\hline \hline \multicolumn{5}{|c|}{$(Q * \eta)$} \\
\hline highest $\mathrm{Q}^{*} \eta$ & 35.79 & 21.98 & 74.85 & 53.10 \\
2nd Q $^{*} \eta$ & 64.22 & 44.50 & 25.15 & 31.40 \\
lowest Q* $\eta$ & $\mathrm{N} / \mathrm{A}$ & 33.52 & $\mathrm{~N} / \mathrm{A}$ & 15.51 \\
\hline \multicolumn{5}{|c|}{$E_{T}$} \\
\hline leading jet & 64.46 & 48.85 & 51.52 & 41.85 \\
2nd jet & 35.54 & 36.35 & 48.48 & 42.22 \\
3rd jet & $\mathrm{N} / \mathrm{A}$ & 14.80 & N/A & 15.93 \\
\hline \multicolumn{5}{|c|}{$\chi^{2}$} \\
\hline Smallest $\chi^{2}$ & 80.85 & 66.84 & 69.89 & 58.59 \\
\hline
\end{tabular}

Table 6.3: Fraction of matched events (jets closest to the generator labeled $b$ particle) as functions of $(Q * \eta), \chi^{2}$, and $E_{T}$. Studies made using MADEVENT $t$-channel and $s$-channel Monte Carlo samples.

Input variables include:

- SECVTX tight tag of each jet: a boolean indicating if the jet is SECVTX tagged.

- $\Delta \chi^{2}: \log \left(\chi^{2}(\right.$ jet 1$\left.)\right)-\log \left(\chi^{2}(\right.$ jet 2$\left.)\right)$ where $\chi^{2}$ (jet) is the smaller of the two values of $\chi^{2}$ associated with a jet, i.e. the values of $\chi^{2}$ calculated by taking that jet as the input to the kinematic solver. The two solutions come from the two possible $P_{z}$ neutrino solutions. The two leading jets are labeled "jet1" and "jet2".

- $\Delta P_{T}: P_{T}($ jet 1$)-P_{T}($ jet 2$)$

- $Q \times \eta: Q_{l e p} \times \eta(j e t 1)$, where $Q_{l e p}$ is the charge of the lepton. This variable takes advantage of the asymmetry of $Q_{l e p} \times \eta($ non $b-j e t)$, see Figure 7.1 in Section 7.2.

Two $b$-choosing algorithms were devised corresponding to the $t$-channel 2 and 3 jet bins.

- Algorithm 1: t-channel 2-jet bin

- If there is exactly one tagged jet, choose the tagged jet.

- If there are two or more tagged jets, choose the tagged jet with the lowest $Q \times \eta$.

This algorithm chooses the correct $b$ from the reconstructed top quark $95 \%$ of the time.

- Algorithm 2: t-channel 3-jet bin.

- If there is exactly one tagged jet, choose the tagged jet. 
- If there are two or more tagged jets, consider only the two leading jets. Choose the jet with the highest combination of $\Delta \chi^{2}$ and $\Delta P_{T}$, $\mathrm{C}$, given by $\mathrm{C}=\left(\Delta \chi^{2}\right)+\left(0.005 \times \Delta P_{T}\right)$. Here $\Delta \chi^{2}$ and $\Delta P_{T}$ are calculated as described above.

This algorithm chooses the correct $b$ from the reconstructed top quark $75 \%$ of the time.

Additionally, in the three jet bin, the choice of the $b$-tagged jet does not uniquely determine the choice of the 'other' jet, which corresponds to the lightflavored quark recoiling from the single top production. Thus, a non- $b$ chooser must be implemented as follows: If there are exactly two tags, the non-tagged jet was assigned to be the other jet, otherwise the more energetic of the two jets that were not picked was chosen to be the other jet. A summary of the 3-jet bin algorithms used is given in Table 6.4.

\begin{tabular}{|l|r|r|r|}
\hline Algorithm & 1 tag & 2 tag & 3 -tag \\
\hline$b$ from top & $b$-tag & $b$-chooser & $b$-chooser \\
\hline 'other' jet & non- $b$ chooser & non-tagged jet & non- $b$ chooser \\
\hline
\end{tabular}

Table 6.4: Choosing algorithms used in the 3-jet bin Likelihood function. Here the $b$-chooser chooses the jet with the highest value of $-\log \chi^{2}+0.005 P_{t}$ out of the leading two jets, and the non- $b$ chooser is the leading jet not chosen as the $b$ from top decay.

\subsection{Neural Net B Tagger}

The Neural Net B Tagger (ANN Tagger) designed to help distinguish c-quark, light flavor and other non- $b$ events from events containing real $b$ hadrons. The Neural Network B Tagger output describes the probability of each event containing a $b$ tag. This variable is used as an input to the multivariate likelihood function analysis, as described in Section 7.

The Neural Net B Tagger consists of a Neural Network, made using the NeuroBayes package [83], that combines a number of secondary vertex quantities and other characteristics of $b$ hadrons into a continuous variable [79]. The ANN Tagger uses a total of 31 input variables. These variables are a mix of kinematic and SECVTX quantities, similar to the list presented here [79]. The final ANN Tagger discriminant is shown in Figure 6.6.

After the creation of the ANN Tagger, the distributions were checked against data control samples to check if correction factors were needed and to evaluate the systematic uncertainties. The correction factors address any modeling differences in the input variables to the ANN Tagger in Monte Carlo samples (used to create the tagger) and in data. For this comparison, two samples were used, a $b$ quark sample and a light quark or mistag sample. 


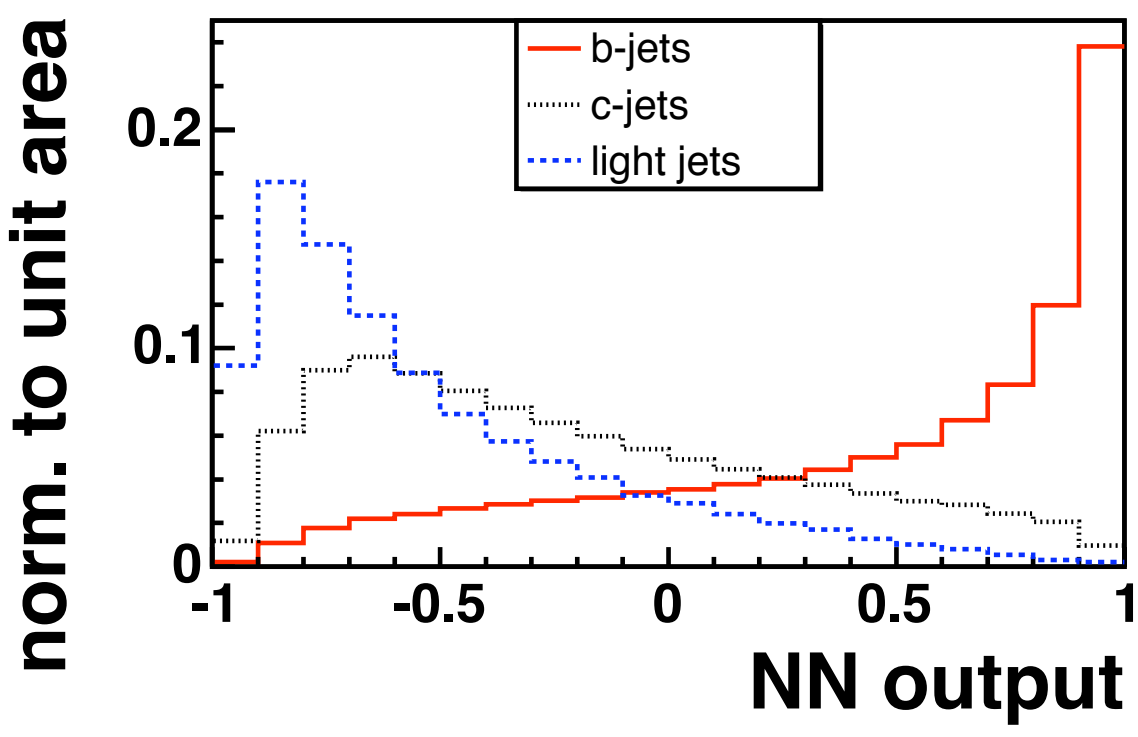

Figure 6.6: Neural Net B Tagger plotted for $b$-quark jets, $c$-quark jets and light-quark jets.

In the case of the $\mathrm{b}$ quark sample, an energetic electron was required to be within the jet (the "electron jet"), and an additional jet was required with opposite $\phi$ direction. This second jet (or "away jet") was used for comparison purposes. Both the electron jet and the away jet were required to have a SECVTX tag. This comparison was done separately for events where the "away jet" was tagged on the first or second pass of the SECVTX algorithm (pass 1/pass 2 verticies). In both cases, it was determined that no correction factor was needed, with the discrepancies being consistent with regions of low statistics [79].

The light quark or mistag data sample was composed of generic dijet samples, and the Monte Carlo sample was composed of PYTHIA diijet samples. Negatively tagged SECVTX jets are used, as described in Section 5.4.1. Since in this case the decay length is by definition negative, the absolute value of decay length was used in place of decay length in this comparison. In this case, the ANN output was seen to be different and a correction function was applied. The correction function was parameterized using some of the variables which parameterize the mistag matrix, see Section 5.4.1. These include $E_{T}, \sum E_{T}$, $|\eta|$ and the number of good tracks in the jet. Separate correction functions were determined for pass 1 and pass 2 SECVTX tags. These functions are shown in Figure 6.7.

To create the ANN Tagger distributions used in a multivariate analysis (like the multivariate likelihood analysis) the light flavor correction was applied to the 

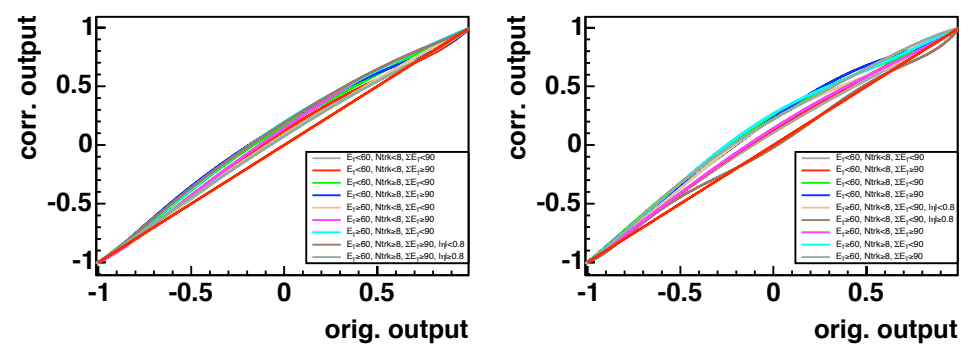

Figure 6.7: Correction functions applied to the Neural Net $b$ Tagger. The functions were computed separately for pass 1 SECVTX veticies (plot 1) and pass 2 SECVTX verticies (plot 2). The functions were parameterized as a function of $E_{T}, \sum E_{T},|\eta|$ and number of good tracks in the jet.

mistag sample only, and no other corrections were applied. A shape systematic for the Neural Network $b$ tag output was created by applying the correction functions to alternate samples. Two systematics were applied, the 'optimistic' correction, where the light flavor correction function was not applied to the mistag jets, and the 'pessimistic' correction where the mistag correction function was applied to $W c \bar{c}$ and $W c$ templates. The implementation of this systematic will be discussed in Section 8.1. 


\section{Likelihood Function}

\subsection{Introduction to Likelihood Functions}

Single top is a challenging signal to observe because it has a relatively low cross section, and also looks more like background events than other processes such as $t \bar{t}$ production. This makes an observation of single top nearly impossible using a cut-based analysis with the current integrated luminosity from the Tevatron. Fortunately, single top events do have some distinguishing features which can be used to help separate single top signal from background.

In $t$-channel single top production, the recoil jet usually travels in the same direction as the parent proton or anti-proton. Moreover, the charge of the final lepton from the $W$ decay can be traced back to the flavor of the parent quarks (i.e. $u, \bar{u}, d$ or $\bar{d}$ ). Multiplying the angle of the recoil or 'non $b$-tagged' jet by the charge of the lepton gives a variable $(Q \times \eta)$ with a distinct asymmetry for t-channel single top production, see Figure 7.1. This asymmetry is not seen for background events, making $Q \times \eta$ an excellent variable to distinguish $t$-channel single top production from backgrounds [84].

Another variable that can be exploited to search for single top is the angle between the lepton momentum and the untagged jet. The cosine of this angle $\left(\cos \theta_{t-\text { chan }}\right)$ is distributed very differently in the $t$-channel compared to backgrounds due to the the near- $100 \%$ polarization of the top quark when it is produced electroweakly [40] [42]. This distinct signature can be seen in Figure 7.1.

However, using any one of these variables alone does not provide enough signal to background separation to differentiate single top from the background with the present luminosities. A better strategy, then, is to combine many of these variables into a multivariate discriminant. A multivariate discriminant can take advantage of many differences between signal and background, producing a final output histogram with much higher signal to background separation than any individual variable.

One such multivariate discriminant is called the multivariate likelihood function. The first step in the construction of a multivariate likelihood function is to choose a number of input variables. The optimization of the likelihood function primarily comes from the choice of these variables. Generally, a large number of variables are considered, and a subset is chosen that yields an optimum signal to background separation. 
Once the input variables are chosen, reference histograms must be created for each variable. Each histogram is composed of $n_{i}$ bins, and the value of each bin is denoted $f_{i j k}$ where $i$ denotes the variable, $j$ is the bin, and $k$ is the event class, either $t$-channel signal, or one of the background types. In this analysis, four background classes are used: $W b \bar{b}, t \bar{t}, W c \bar{c} / W c$ and $\mathrm{W}+\mathrm{LF}$. These histograms are normalized to unit area, i.e. $\sum_{j=1}^{n_{i}} f_{i j k}=1$. Separate histograms are calculated using signal and important background distributions.

The likelihood function can then be computed by evaluating which bin $j$ a given event falls in the distribution of variable $i$. This is used to compute the variable $p_{i k}$ as follows:

$$
p_{i k}=\frac{f_{i j k}}{\sum_{m=1}^{5} f_{i j m}},
$$

The multivariate likelihood function, $\mathcal{L}$, can then be computed as follows:

$$
\mathcal{L}_{\text {signal }}=\frac{\prod_{i=1}^{n_{\text {var }}} p_{i, \text { signal }}}{\sum_{m=1}^{5} \prod_{i=1}^{n_{\text {var }}} p_{\text {im }}} .
$$

The multivariate likelihood function has some advantages and disadvantages compared to other types of multivariate analysis. The likelihood function is relatively straightforward to code and implement, so a number of different configurations can be tested with relative ease. The calculation of the likelihood function also takes a very small amount of time compared to other multivariate techniques (such as the Matrix Element technique also used at CDF). One disadvantage to the multivariate likelihood function technique is it does not take into account the correlations between the input variables. Other multivariate methods that do take into account such correlations, notably Neural Networks and Decision Trees, can be more sensitive than the multivariate likelihood function. However, with a clever choice of variables, the multivariate likelihood function technique is a strong analysis in its own right.

Two likelihood functions were computed, $\mathcal{L}_{2 t}$ calculated using the 2-jet bin and $\mathcal{L}_{3 t}$ calculated using the 3 -jet bin. Each jet bin uses a separate $b$-tagging algorithm, as described in Section 6.2, and the two likelihood functions were optimized separately.

\subsection{The Two-Jet Bin}

The 2-jet bin Likelihood Function was initially optimized using angular and energy related quantities as well as the MadGraph matrix elements described in Section 6.1.1. In calculating these variables, the 2 -jet bin t-channel $b$-selection algorithms were used, as described in Section 6.2. When reconstructing the neutrino, the smaller of the two $\left|p_{z, \nu}\right|$ solutions was chosen. This choice was found to yield the correct $\left|p_{z, \nu}\right|$ solution $77 \%$ of the time in single top Monte Carlo. Variables considered included: 
- $H_{T}$ : The scalar sum of the transverse energies of the two jets, the lepton, and the missing transverse energy.

- $Q \times \eta$ : The charge of the lepton times the pseudorapidity of the jet which is not $b$-tagged.

- $\cos \theta_{t \text {-chan }}$ : the cosine of the angle between the lepton momentum and the untagged jet in the top decay frame.

$-\cos \theta_{s-\text { chan }}$ : The cosine of the angle between the lepton and beam in the top frame.

- $M_{j j}$ : The invariant mass of the two jets.

- $P_{T}$ of the highest and lowest momentum jets

$-\left|E_{T}\right|$

$-P_{T}($ jet $1+$ jet 2$):$ The sum of the transverse energies of the two jets.

- $\log \left(\mathrm{ME}_{t-\text { chan }}\right):$ The $\log$ of the MadGraph matrix element computed using the constrained four-vectors of the $b$, the $\ell$ and the $\nu$, assuming $t$-channel signal production.

- $\log \left(\mathrm{ME}_{s-\text { chan }}\right):$ The difference in the $\log$ of the MadGraph matrix element calculated assuming $s$-channel signal production.

- $\log \left(\mathrm{ME}_{W b b}\right)-\log \left(\mathrm{ME}_{t-\mathrm{chan}}\right):$ The MadGraph matrix elements calculated assuming $W b \bar{b}$ background production and $t$-channel signal production respectively.

$-\cos \theta_{W j j}$ : The cosine of the angle between the $W$ and dijet momentum.

- $M_{l \nu b}$ : A hybrid reconstructed top mass constructed using the raw reconstructed value of $P_{b}$, the momentum of the $b$ quark, and $E_{T}$ as the transverse momentum of the neutrino, while taking $p_{z, \nu}$ from the kinematic solver.

- $\chi_{t}^{2}: \chi^{2}$ output from the kinematic solver 6.1.1 using the 2-jet bin t-channel selection algorithms.

The likelihood function was optimized by creating an initial likelihood function using $Q \times \eta, H_{T}$ and $M_{l \nu b}$. A likelihood function with good separation will have the feature that the signal distribution peaks nearest to one (the last bin) and the background peaks near to zero (the first bin). This means the last bin is the most sensitive region to single top production, and the signal to background separation in the last bin indicates the strength of the likelihood function in separating signal from background. With this in mind, variables were then added by selecting the variable which produced a likelihood function which had the highest signal $(s)$ to square root of the background $(b)$ ratio, 
$s / \sqrt{b}$, in the last bin. This was repeated until the improvement produced by adding further variables was minimal, which resulted in a list of six variables. Later, the Neural Network $b$-tagger was added to the list. This change was needed simply because the Neural Network $b$-tagger was not available when the initial set of input variables was chosen, but provides a large increase to the value of $s / \sqrt{b}$ of the last bin of the final likelihood. Finally, the $\chi_{t}^{2}$ variable was substituted for $M_{l \nu b}$ in this analysis, because the $\chi_{t}^{2}$ variable essentially encodes the same mass information as the top mass variable. For double tagged events, $\mathcal{L}_{t 2}$ was set to zero. This designation helps separate $t$-channel signal from $W b \bar{b}$ and $t \bar{t}$ backgrounds, as only about $1 \%$ of $t$-channel events contain two SECVTX tags, compared to $13 \%$ of $W b \bar{b}$ and $19 \%$ of $t \bar{t}$ events. The final list of seven input variables is given below:

$-H_{T}$

$-Q \times \eta$

$-\cos \theta_{t-\text { chan }}$

$-M_{j j}$

- $\chi_{t}^{2}$, output from the kinematic solver for the $t$-channel combination described in Section 6.1.1

$-\log \left(\mathrm{ME}_{t-\mathrm{chan}}\right)$

- ANN $b$-tag, the output of the Artificial Neural Network $b$-tagger for the choice of $b$-jet

Figures 7.1 and 7.4 show normalized distributions of the input variables for the $s$ - and $t$-channel signals as well as the dominant backgrounds.

A template histogram for the $t$-channel likelihood function is given in Fig. 7.5. A stacked plot of the final 2-jet bin likelihood output is shown in Figure 7.6. In this plot, the data falls slightly low in the bins of the likelihood function nearest one, suggesting that the final result will fit a lower than expected single top cross section. 
CDF Run II Preliminary, $2.2 \mathrm{fb}^{-1}$

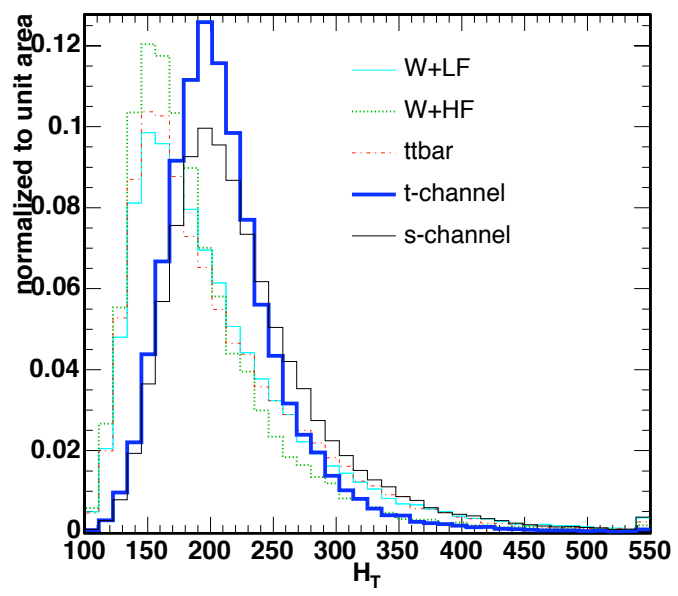

CDF Run II Preliminary, $2.2 \mathrm{fb}^{-1}$

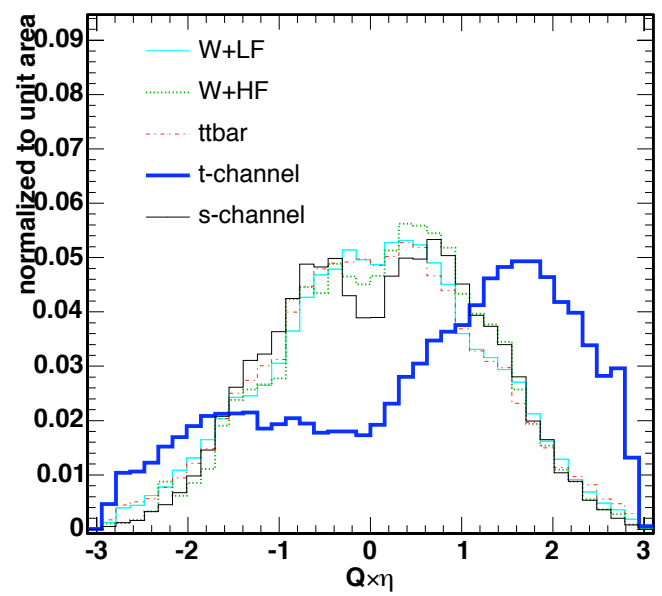

Figure 7.1: $H_{T}$ and $Q \times \eta$, used as input variables to the $t$-channel 2-jet bin likelihood function. 


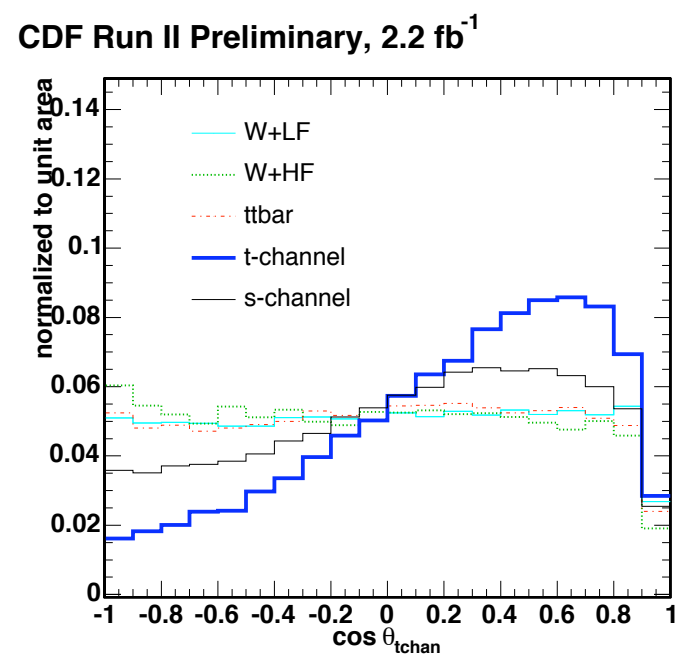

CDF Run II Preliminary, $2.2 \mathrm{fb}^{-1}$

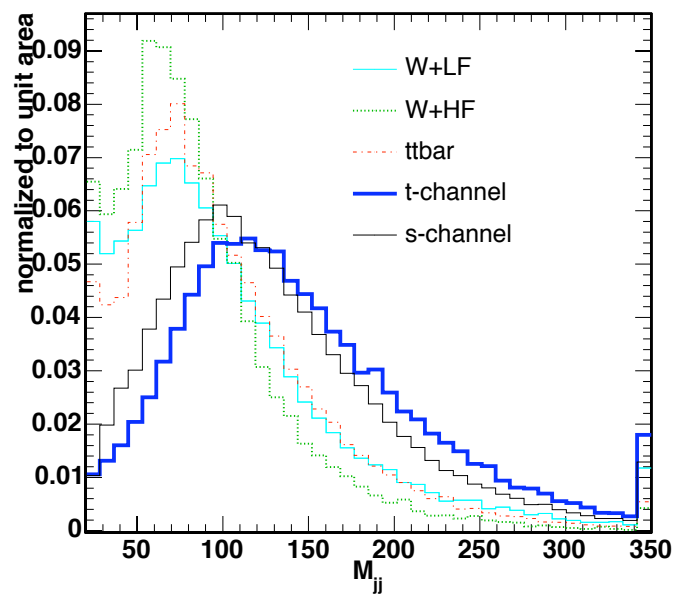

Figure 7.2: $\cos \theta_{t \text {-chan }}$ and $M_{j 1 j 2}$, used as input variables to the $t$-channel 2-jet bin likelihood function. 


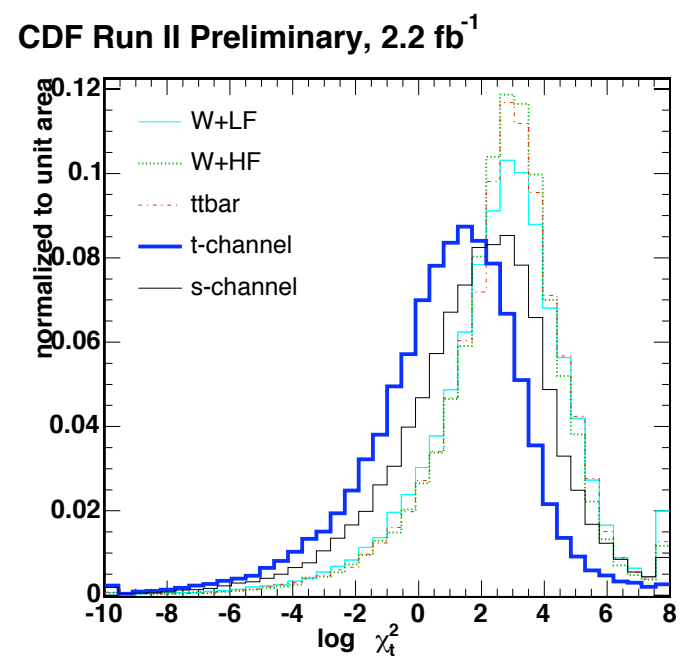

CDF Run II Preliminary, $2.2 \mathrm{fb}^{-1}$

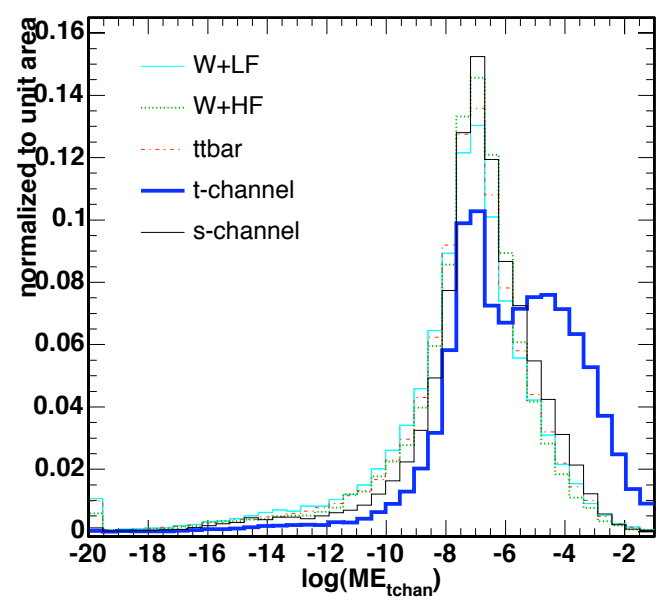

Figure 7.3: $\chi_{t}^{2}$ and $\mathrm{ME}_{t-\text { chan }}$, used as input variables to the $t$-channel 2-jet bin likelihood function. 
CDF Run II Preliminary, $2.2 \mathrm{fb}^{-1}$

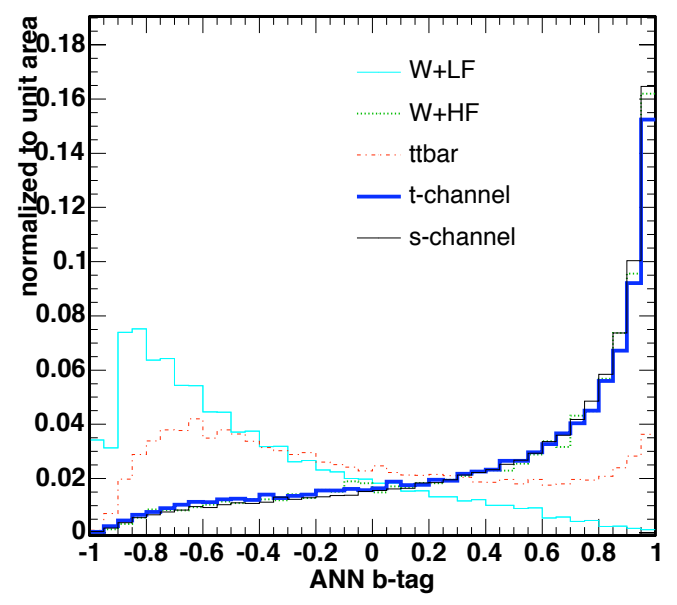

Figure 7.4: ANN $b$-tag, used as input variables to the $t$-channel 2-jet bin likelihood function. 
CDF Run II Preliminary, $2.2 \mathrm{fb}^{-1}$

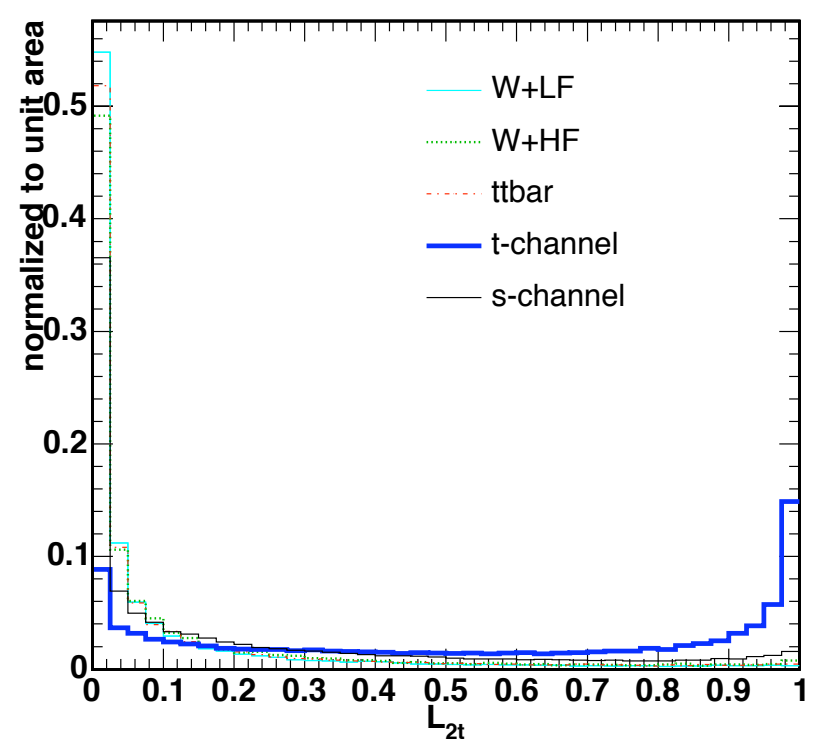

CDF Run II Preliminary, $2.2 \mathrm{fb}^{-1}$

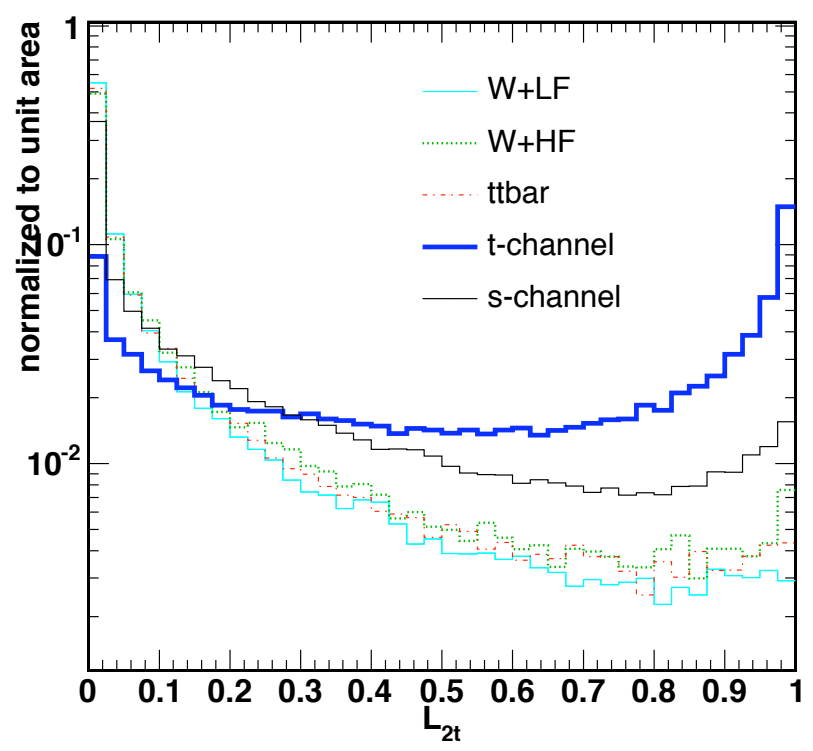

Figure 7.5: Template distribution of the $t$-channel 2-jet bin likelihood function on a linear scale (above) and log scale (below). 


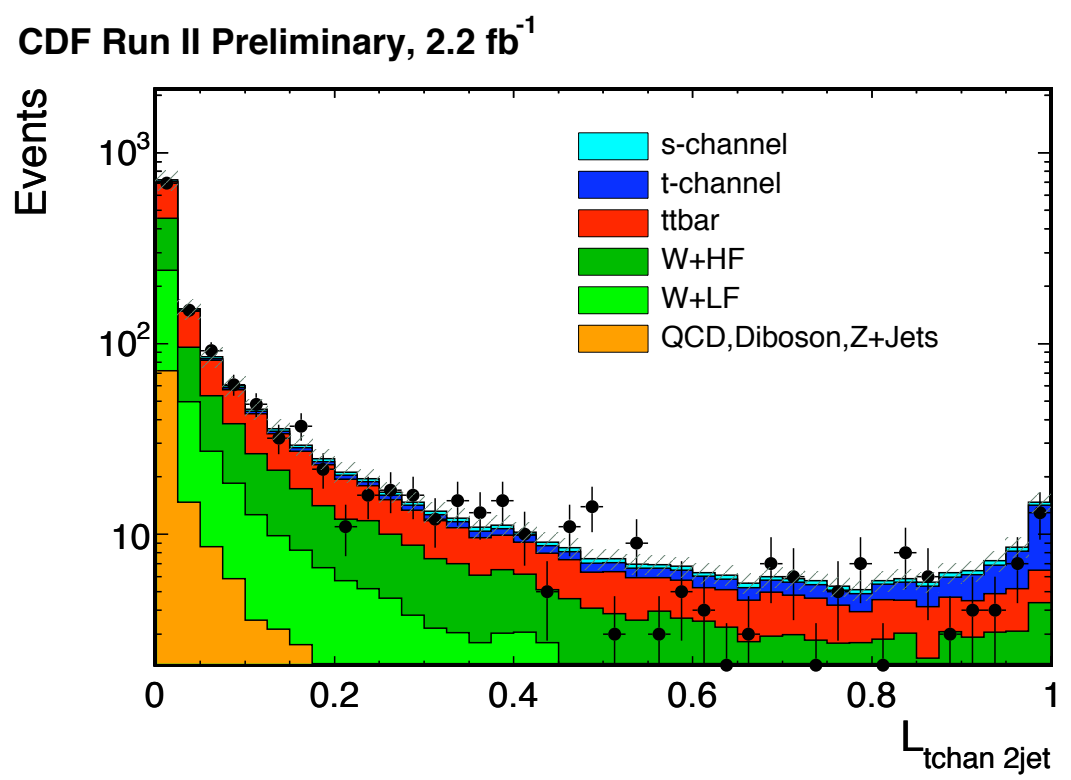

CDF Run II Preliminary, $2.2 \mathrm{fb}^{-1}$

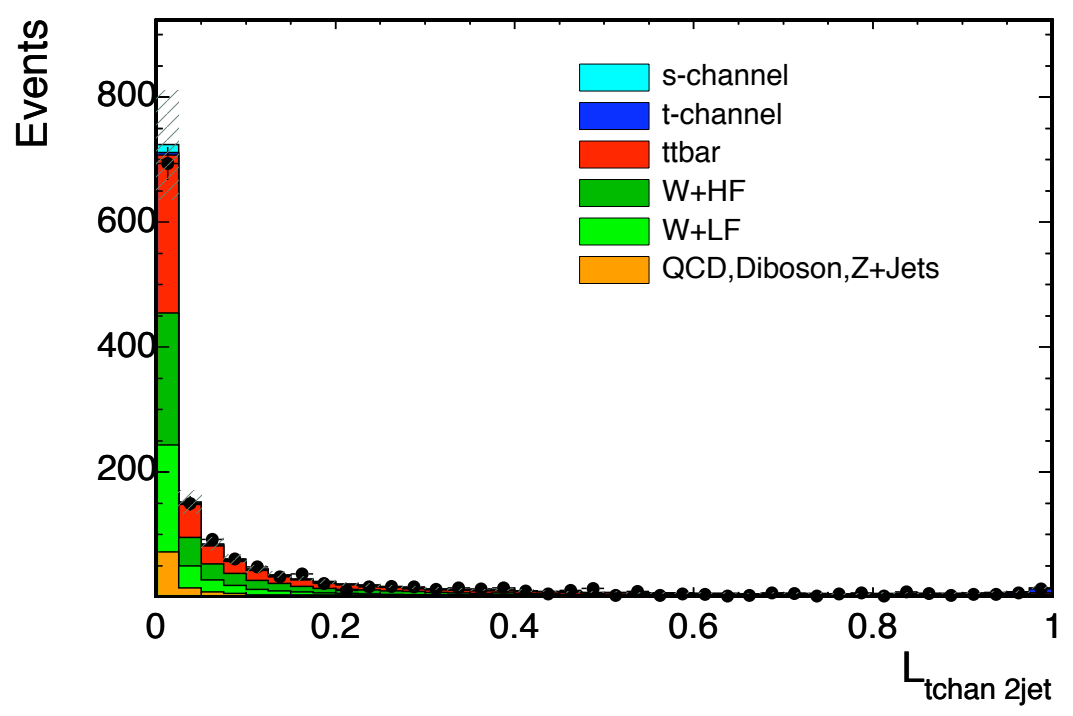

Figure 7.6: 2-jet bin $t$-channel likelihood function $\mathcal{L}_{2 t}$, shown on linear and log scales. Data is compared to the prediction with each component normalized to its predicted value. 


\subsection{The Three-Jet Bin}

With the presence of a third jet in each event, a larger number of reconstructed variables is possible. Therefore, a large number of variables were considered when constructing the 3 -jet bin likelihood function. In calculating these variables, the 3 -jet bin $t$-channel $b$-selection algorithms are used, as described in Section 6.2. When reconstructing the neutrino, the smaller of the two $\left|p_{z, \nu}\right|$ solutions is chosen.

Variables considered in the 3-jet bin included angular and energy related quantities for all three jets, MadGraph matrix elements, as described in Section 6.1.1, the ANN b-tag described in Section 6.3, and variables calculated using the Kinematic Solver (Section 6.1.1), such as $\chi_{s}^{2}$. In total, the following variables in the 3 -jet bin were considered:

- Number of SECVTX $b$-tags

- Scalar sum of the jet energies

- $\eta$ of the reconstructed $\mathrm{W}$, using the neutrino choosing algorithm outlined above

- $P_{T}$ of the lepton

- $E_{T}$ of the leading, second and third jets

- $\Delta R_{12}, \Delta R_{13}, \Delta R_{23}, \Delta R$ of all possible jet combinations

- Lowest $\Delta R$ of any two jets

- Highest $\Delta R$ of any two jets

- Sum of the jet pseudorapidities

- $\eta$ of jet1, jet2, and jet3

- cosine of the angle between any combination of jets in the top rest frame

- $Q \times \eta$, the charge of the 'other' jet, chosen as described above, times the $\eta$ of the lepton

$-\cos \theta_{s-\text { chan }}$

- $\log \left(\mathrm{ME}_{t-\text { chan }}\right)$, the t-channel MadGraph matrix element

- $\log \left(\mathrm{ME}_{s-\text { chan }}\right)$, the s-channel MadGraph matrix element

- $\chi_{s}^{2}$, the output of the kinematic solver for the choice of $b$-jet

- $M_{j j}$ of all two jet combinations $(12,23$, or 13$)$

- ANN $b$-tag, the output of the Artificial Neural Network $b$-tagger for the choice of $b$-jet 
- $H_{T}$, with and without the lowest momentum jet

- $\phi$ of jet1, jet2 and jet3

- Sphericity, calculated from the three jets and lepton

- Aplanarity, calculated from the three jets and lepton

The variables used to compute the $t$-channel likelihood function in the threejet bin, $\mathcal{L}_{3 t}$, were optimized in a manner similar to that of $\mathcal{L}_{2 t}$. In ranking a given set of variables, the quantity $s / \sqrt{b}$ for the highest bins was used. Here, $s / \sqrt{b}$ for the highest bins was calculated by summing the signal and background for a given likelihood function, $\mathcal{L}_{\text {test }}$, starting with last bin (see Section 7.2) and adding bins until $s / \sqrt{b}$ for all bins used began to decrease. The quantity $s / \sqrt{b}$ for the highest bins is thus the highest $s / \sqrt{b}$ for any set of bins of $\mathcal{L}_{\text {test }}$.

Likelihood functions were iteratively constructed by adding one variable at a time to the variable set. The first variable chosen was the variable which made the single variable likelihood function, $\mathcal{L}_{\text {test }}$, with the largest $s / \sqrt{b}$ for the highest bins. Then, variables were added to the variable set in order of their contribution to the overall signal and background separation. This procedure was continued until adding new variables no longer produced an increase in $s / \sqrt{b}$ for the highest bins.

The variables chosen in this manner, in order of their importance are:

$-m_{\ell \nu b}$

- ANN $b$-tag output

- The number of $b$-tagged jets

$-Q \times \eta$

- The smallest $\Delta R$ of any two jets

- $M_{j j}$ of the two jets not chosen to be the $b$ from top decay

$-\cos \theta_{t-\text { chan }}$

- $P_{T}$ of the lowest momentum jet.

- $\eta$ of the reconstructed $W$ boson

- $p_{T}$ of the jet chosen to be the $b$ from top decay

This procedure for creating a likelihood function might be highly sensitive to the initial variable chosen. To explore this feature a likelihood function was made with all combinations of two variables from the set of variables considered for the 3 -jet bin. The two variables which yielded the likelihood function with the highest $s / \sqrt{b}$ for the highest bins were chosen. These variables were ANN $b$-tag output and the number of $b$-tagged jets. Both ANN $b$-tag and the number 
of $b$-tagged jets variables are in the top three variables chosen by the algorithm of adding one variable at a time, which suggests that our list has some robustness. Further studies could made by testing variable lists with varied starting parameters, but this is not an issue of correctness, but of optimization.

Figures 7.7 through 7.11 show normalized distributions of the selected input variables for the $s$ - and $t$-channel signal as well as the dominant backgrounds.

CDF Run II Preliminary, $2.2 \mathrm{fb}^{-1}$

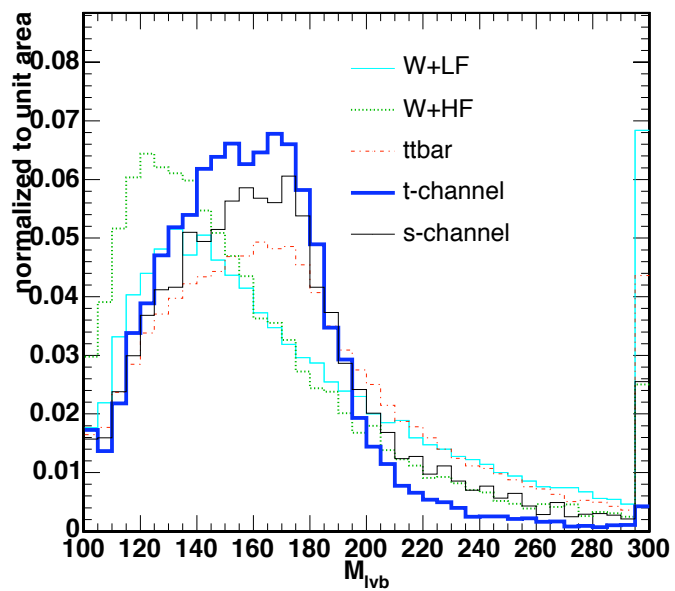

CDF Run II Preliminary, $2.2 \mathrm{fb}^{-1}$

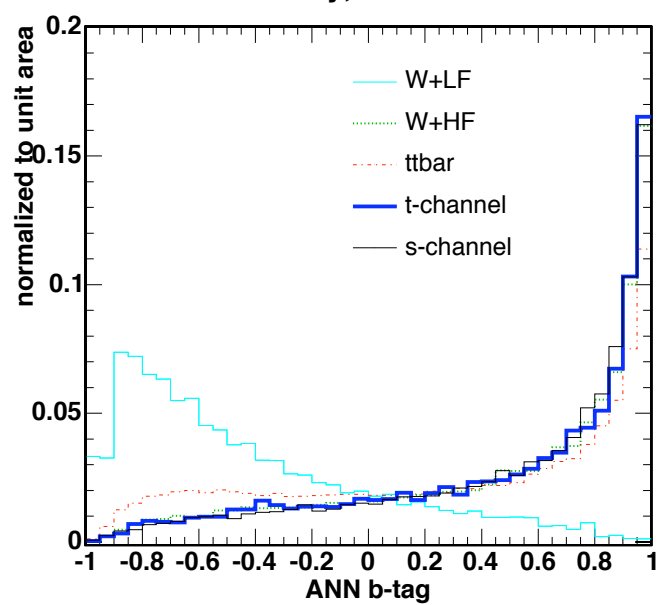

Figure 7.7: $M_{l \nu b}($ tchan) and $N N(b)$, used as input variables to the $t$-channel 3 -jet bin likelihood function. 
CDF Run II Preliminary, $2.2 \mathrm{fb}^{-1}$

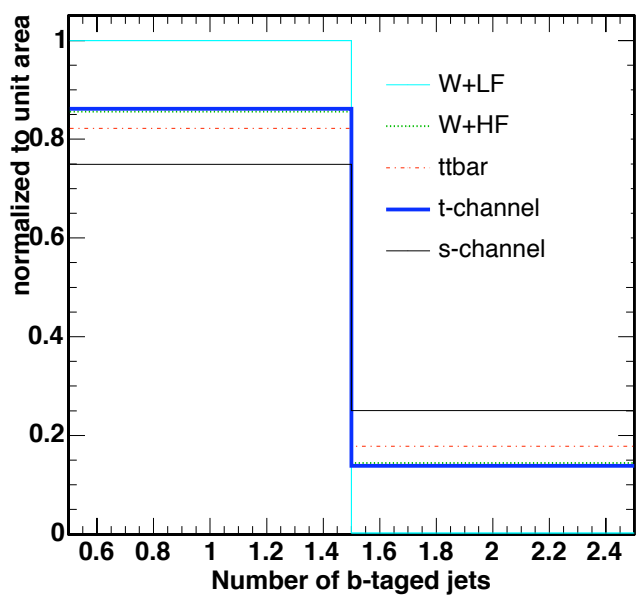

CDF Run II Preliminary, $2.2 \mathrm{fb}^{-1}$

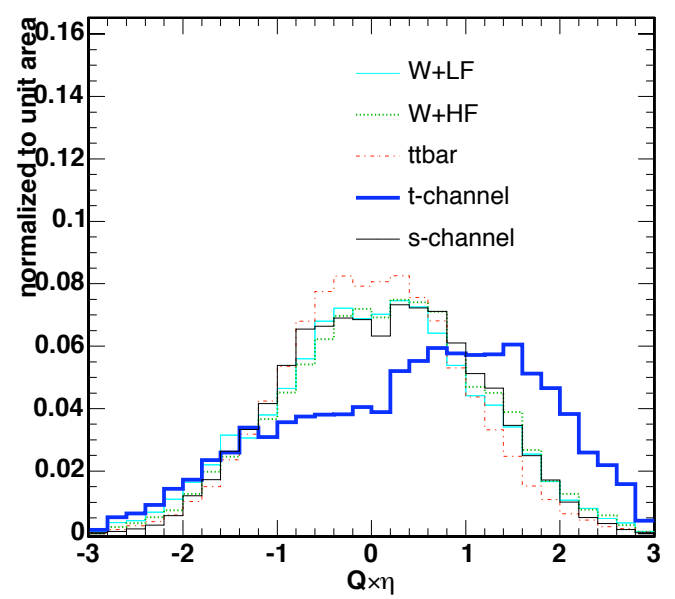

Figure 7.8: The number of tagged jets and $Q \times \eta$ of the untagged jet, used as input variables to the $t$-channel 3 -jet bin likelihood function. In the plot of the number of tagged jets, jets with three tags are grouped in the three jet bin, because there are very few 3 -tag events and grouping this bin by itself would cause very low Monte Carlo statistics for the signal and background samples in this bin. 
CDF Run II Preliminary, $2.2 \mathrm{fb}^{-1}$

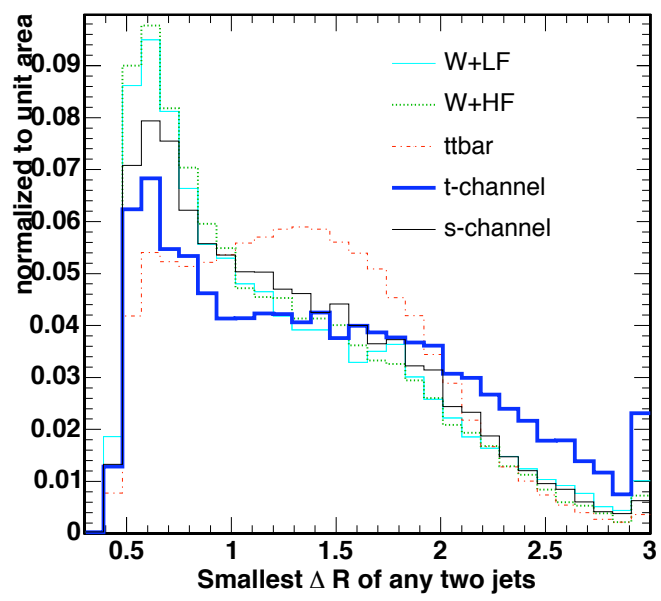

CDF Run II Preliminary, $2.2 \mathrm{fb}^{-1}$

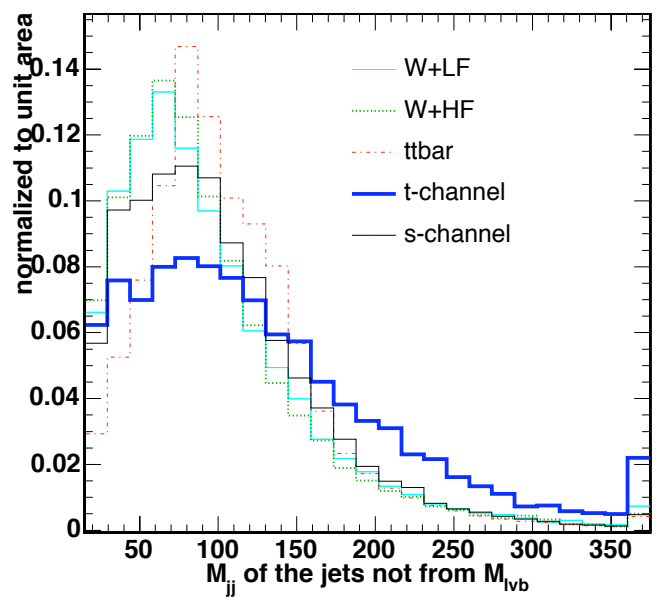

Figure 7.9: $\Delta R$ of the two closest jets and the dijet invariant mass of the two non-tagged jets, used as inputs to the $t$-channel 3-jet bin likelihood function. 


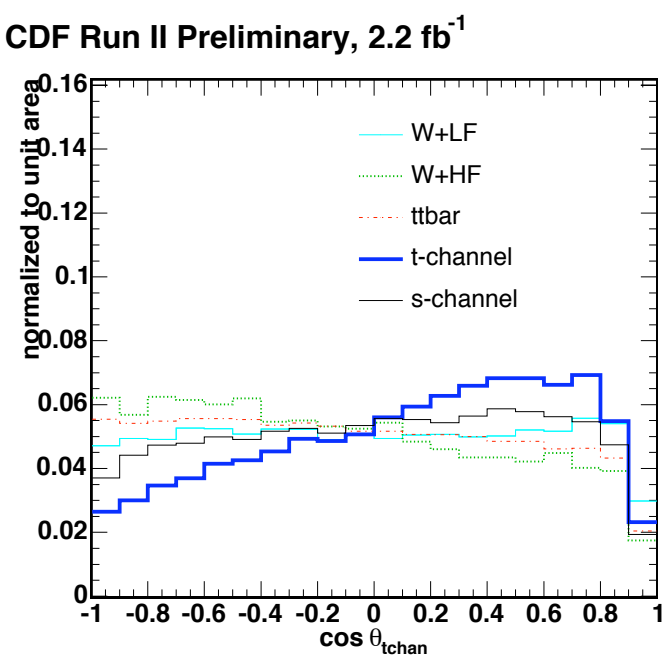

CDF Run II Preliminary, $2.2 \mathrm{fb}^{-1}$

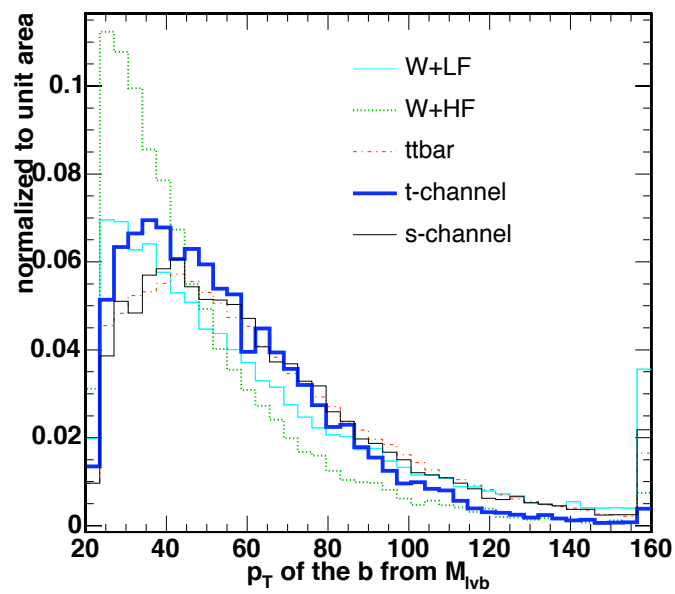

Figure 7.10: $\cos (\theta)_{t c h a n}$ of the two non-tagged jets and $p_{T}$ of the jet chosen to be $\mathrm{b}$ from top decay, used as inputs to the $t$-channel 3-jet bin likelihood function. 


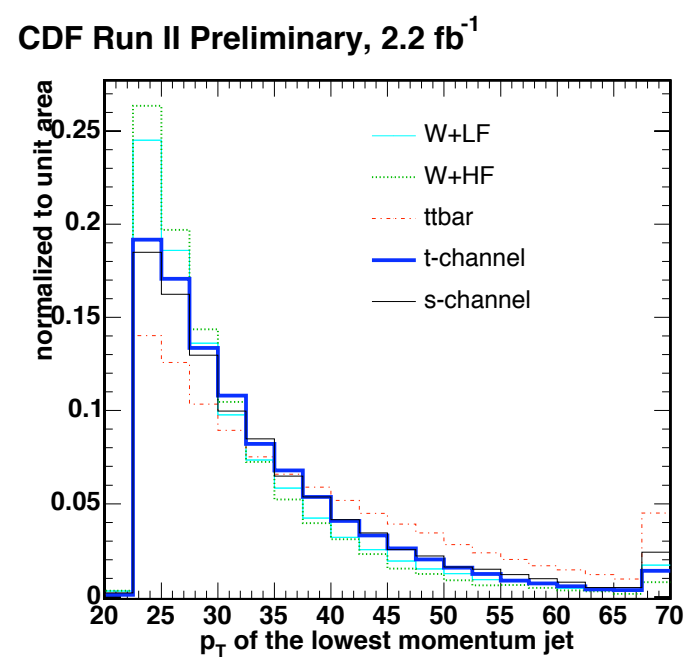

CDF Run II Preliminary, $2.2 \mathrm{fb}^{-1}$

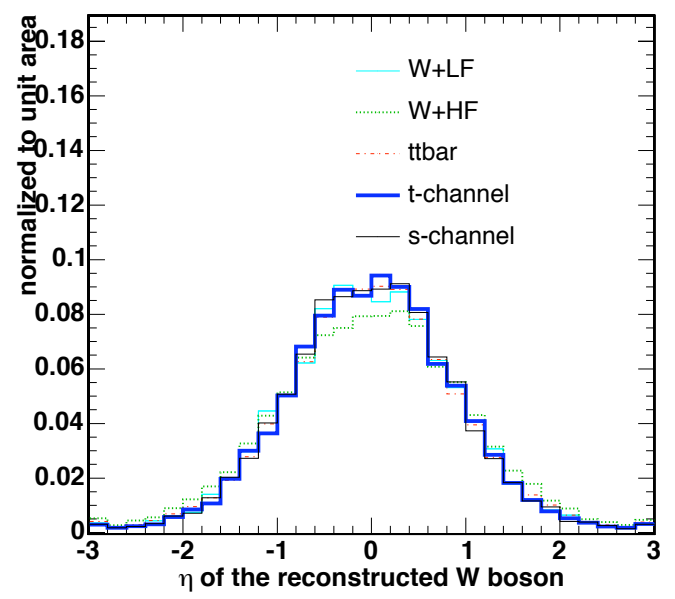

Figure 7.11: $P_{T}$ of the lowest momentum jet and $\eta$ of the reconstructed $W$ boson, which are used as inputs to the $t$-channel 3-jet bin likelihood function. 
$\mathcal{L}_{\text {final }}$ created by this procedure was found to have several high $s / \sqrt{b}$ bins where there was a very small background prediction. This was undesirable, since the background is not well predicted in bins with very low background content because of limited Monte Carlo statistics. A simple transformation was made by taking the $\sin \left(\pi / 2 \cdot \mathcal{L}_{\text {final }}\right)$. A Taylor expansion of $\sin (\pi / 2 \cdot x)$ around one shows that, to first order $\sin (\pi / 2 \cdot x) \approx 1$ for $x \approx 1$. Thus, this simple transformation has the effect of grouping the high $s / \sqrt{b}$ bins together (which have $\mathcal{L} \approx 1$ ), so that in the final distribution there is enough Monte Carlo statistics for an accurate prediction of the background in each bin.

Figure 7.12 shows the normalized templates for the likelihood function $\mathcal{L}_{3 t}$. A stacked plot of the final 3-jet bin likelihood output is shown in Figure 7.13. As with the two jet plot, the data falls slightly low in the bins of the likelihood function nearest one, suggesting that the final result will fit a lower than expected single top cross section.
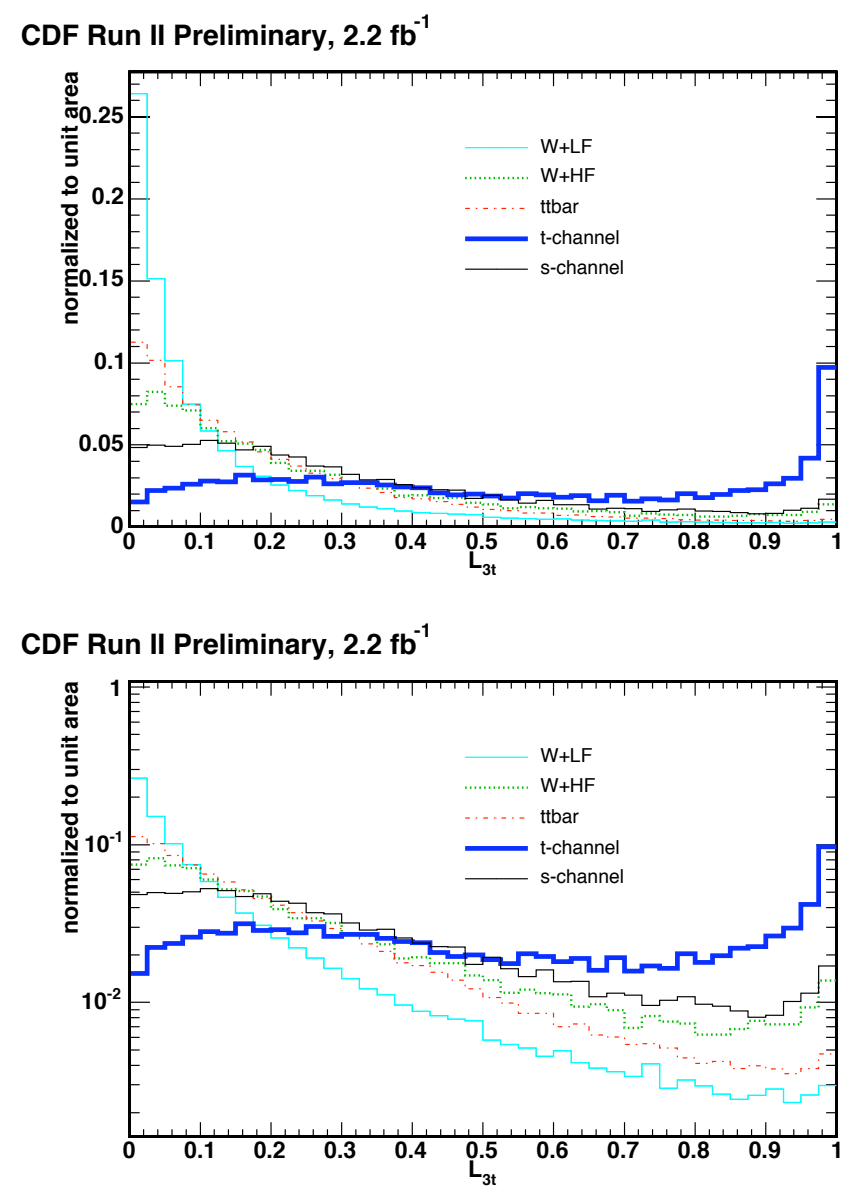

Figure 7.12: Templates for the 3-jet bin's $t$-channel likelihood function $\mathcal{L}_{3 t}$, shown on a linear and log scale. 


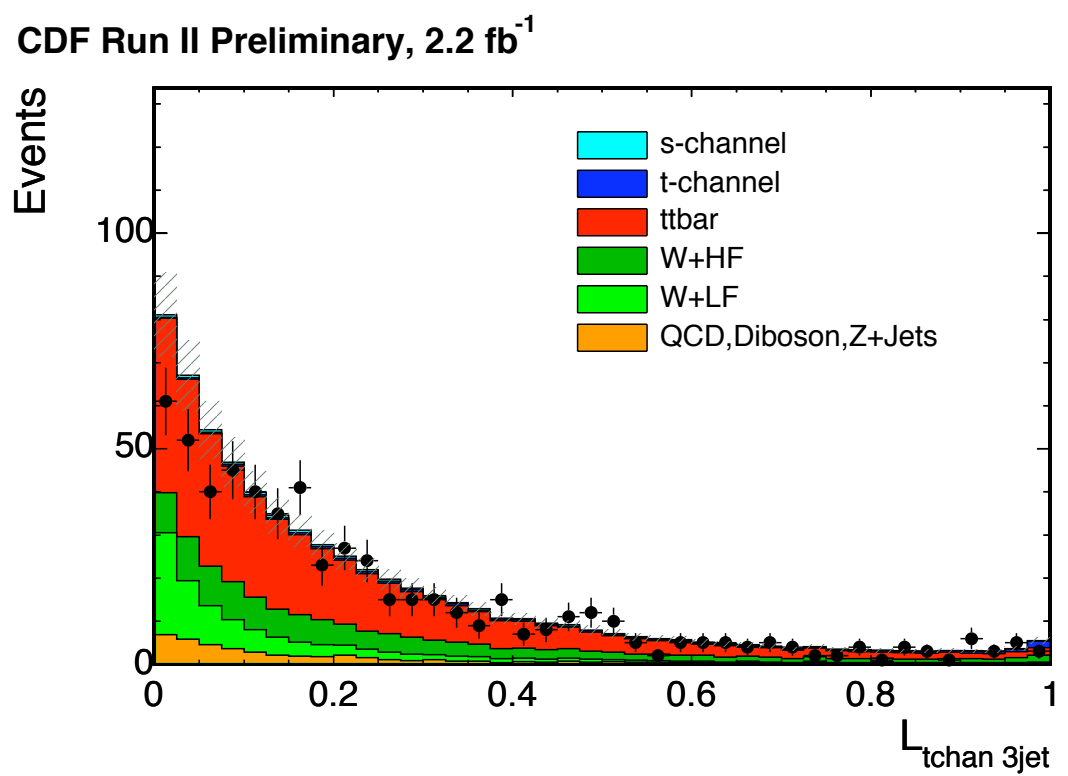

CDF Run II Preliminary, $2.2 \mathrm{fb}^{-1}$

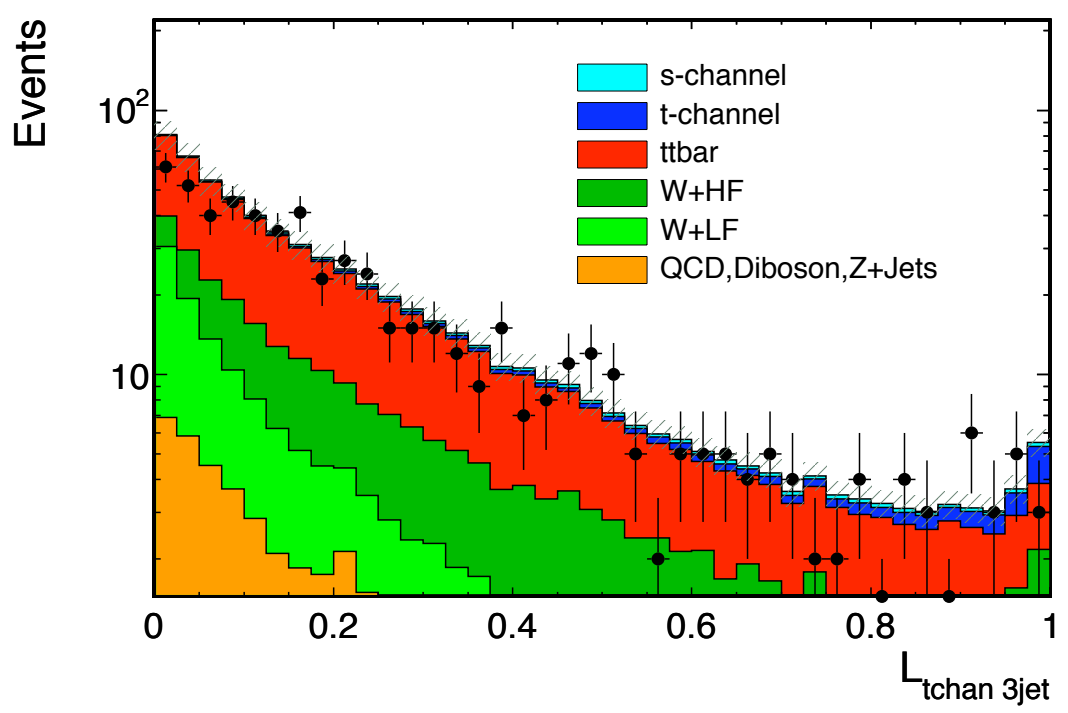

Figure 7.13: 3 -jet bin $t$-channel likelihood function $\mathcal{L}_{3 t}$, shown on linear (above) and $\log$ scales (below). 


\subsection{Variable Check Plots}

Check plots were made of many variables, including the input variables to both the 2-jet and 3-jet likelihood functions. For these checks, the Monte Carlo samples were used as described in Section 5. For the 2-jet and 3-jet bins with a SECVTX tagging requirement ('tag' bins) the components were normalized to the predicted vales from Section 5.5.2, including signal samples. For the 2-jet and 3-jet 'taggable' bins, variables were plotted in the pretag sample. For the pretag sample, the various components were weighted by their pretag distribution, and the final Monte Carlo plot was normalized to the data prediction. Check plots are shown below, organized into input variables for the 2-jet likelihood function, input variables for the 3-jet likelihood function and other input variables.

Modeling checks were made for check plots in the 2-jet and 3-jet tag bins. These modeling checks included KS and $\chi^{2}$ tests, both performed without systematics included as well as a visual modeling check. The results of the KS and $\chi^{2}$ checks are given in Tables 7.1and 7.2. In the 2-jet and 3-jet taggable bins, the statistics are very high, so modeling tests without systematics included lead to deceptively low results. In these cases, plots were examined visually for modeling discrepancies.

In this manner, a few modeling discrepancies were found. The largest discrepancies can be grouped into two categories, modeling of the angles between the jets, and modeling of the high $\eta$ regions of the jets. Modeling systematics were taken for these discrepancies if there was some suggestion of mis-modeling in the tag bin and the high statistics taggable bin showed mis-modeling. Systematics were taken for variables with low correlations in an attempt not to over-estimate modeling systematics.

The mis-modeling in the angle between the two jets is shown most explicitly in the plot of $\Delta R(j j)$ in the two jet bin, which has a low KS value (0.005), and also exhibits mis-modeling in the pretag 2-jet plot. Related distributions also exhibit a less pronounced mis-modeling. A shape systematic was taken for this distribution in the 2-jet bin, which will be further described in Section 8.1. In the 3 -jet bin no systematic was taken because although the KS test is low in the 3-jet tag plot, the 3 -jet taggable plot does not exhibit the mis-modeling of the 2-jet taggable plot (Figure 7.31).

The mis-modeling of the high $\eta$ regions of the lowest momentum jet can be seen in the 2 and 3-jet taggable plots (Figure 7.41). A shape systematic was taken for the $\eta$ of the lowest $\eta$ jet in both the 2-jet and 3-jet bins.

Several other modeling systematics were also considered. These were not justified by specific mis-modeled distributions, but rather known detector and Monte Carlo effects, such as a mistag mis-modeling, jet energy scale uncertainty, and initial and final state radiation uncertainty. These will be discussed in detail in Section 8.1. 


\begin{tabular}{|c|c|c|}
\hline Variable & 2-jet KS & 3-jet KS \\
\hline \hline 2-jet LF inputs & & \\
\hline NN B-Tag & 0.988 & 0.051 \\
$\ln \left(\chi^{2}\right)$ & 0.058 & 0.971 \\
$\cos \left(\theta_{t c h a n}\right)$ & 0.011 & 0.971 \\
$H_{T}$ & 0.194 & 0.344 \\
$M_{j j}$ & 0.035 & 0.087 \\
$Q \times \eta$ & 0.094 & 0.079 \\
$\ln \left(M E_{t c h a n}\right)$ & 0.063 & 0.17 \\
\hline \hline 3 -jet LF inputs & & \\
\hline$m_{\ell \nu b}$ & 0.093 & 0.876 \\
Number of tags & 0.478 & 0.074 \\
$\Delta R($ ji) $($ smallest $)$ & 0.005 & 0.052 \\
$M_{j j}$ (non top jet) & 0.035 & 0.078 \\
Lowest jet $P_{T}$ & 0.998 & 0.255 \\
$\eta$ of the reconstructed $W$ boson & 0.387 & 0.27 \\
$p_{T} b$ from top & 0.407 & 0.858 \\
\hline \hline Other Variables & & \\
\hline$\eta$ of the lowest momentum jet & 0.675 & 0.608 \\
$\eta$ of jet 1 & 0.961 & 0.444 \\
$\eta$ of jet 2 & 0.675 & 0.377 \\
$\eta$ of jet 3 & N/A & 0.608 \\
$\phi$ of jet 1 & 0.777 & 0.848 \\
$\phi$ of jet 2 & 0.729 & 0.446 \\
$\phi$ of jet 3 & N/A & 0.495 \\
$P_{T}$ of the lepton & 0.229 & 0.520 \\
\hline
\end{tabular}

Table 7.1: KS values for kinematic variables 


\begin{tabular}{|c|c|c|}
\hline Variable & 2-jet $\chi^{2} / \mathrm{DOF}$ & 3-jet $\chi^{2} / \mathrm{DOF}$ \\
\hline \hline 2-jet LF inputs & & \\
\hline NN B-Tag & $44.00 / 40$ & $42.98 / 40$ \\
$\ln \left(\chi^{2}\right)$ & $83.82 / 40$ & $41.43 / 40$ \\
$\cos \left(\theta_{\text {tchan }}\right)$ & $21.59 / 20$ & $17.21 / 20$ \\
$H_{T}$ & $71.57 / 40$ & $38.35 / 40$ \\
$M_{j j}$ & $51.43 / 40$ & $53.87 / 40$ \\
$Q \times \eta$ & $37.62 / 40$ & $62.72 / 40$ \\
$\ln \left(M E_{\text {tchan }}\right)$ & $30.5 / 30$ & $25.7 / 30$ \\
\hline \hline 3 -jet LF inputs & & \\
\hline$m_{\ell \nu b}$ & $62.07 / 40$ & $50.03 / 40$ \\
Number of tags & $3.11 / 2$ & $9.47 / 2$ \\
$\Delta R($ jj) $($ smallest $)$ & $37.32 / 40$ & $40.62 / 40$ \\
$M_{j j}($ non $t o p$ jet $)$ & $51.42 / 40$ & $27.70 / 40$ \\
Lowest jet $P_{T}$ & $23.92 / 40$ & $54.24 / 40$ \\
$\eta$ of the reconstructed $W$ boson & $40.06 / 40$ & $32.96 / 40$ \\
$p_{T} b$ from top & $48.33 / 40$ & $59.73 / 40$ \\
\hline \hline Other Variables & & \\
\hline$\eta$ of the lowest momentum jet & $23.61 / 40$ & $23.54 / 40$ \\
$\eta$ of jet 1 & $48.86 / 40$ & $49.28 / 40$ \\
$\eta$ of jet 2 & $23.61 / 40$ & $23.54 / 40$ \\
$\eta$ of jet 3 & $\mathrm{~N} / \mathrm{A}$ & $23.54 / 40$ \\
$\phi$ of jet 1 & $27.44 / 40$ & $27.24 / 40$ \\
$\phi$ of jet 2 & $27.46 / 40$ & $29.81 / 40$ \\
$\phi$ of jet 3 & $\mathrm{~N} / \mathrm{A}$ & $35.50 / 40$ \\
$P_{T}$ of the lepton & $116.57 / 80$ & $96.63 / 80$ \\
\hline
\end{tabular}

Table 7.2: $\chi^{2} /$ DOF Values for kinematic variables 


\subsection{1 $\quad \mathcal{L}_{2 t}$ Input Variables}

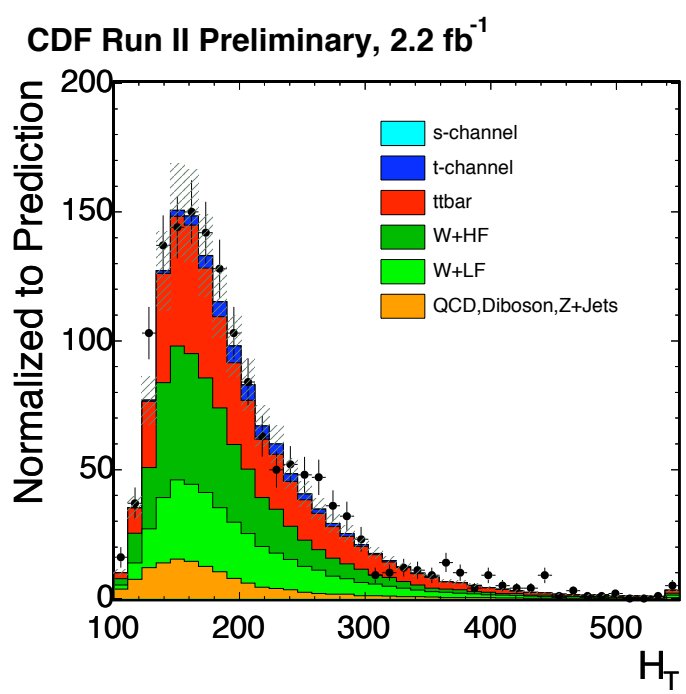

CDF Run II Preliminary, $2.2 \mathrm{fb}^{-1}$

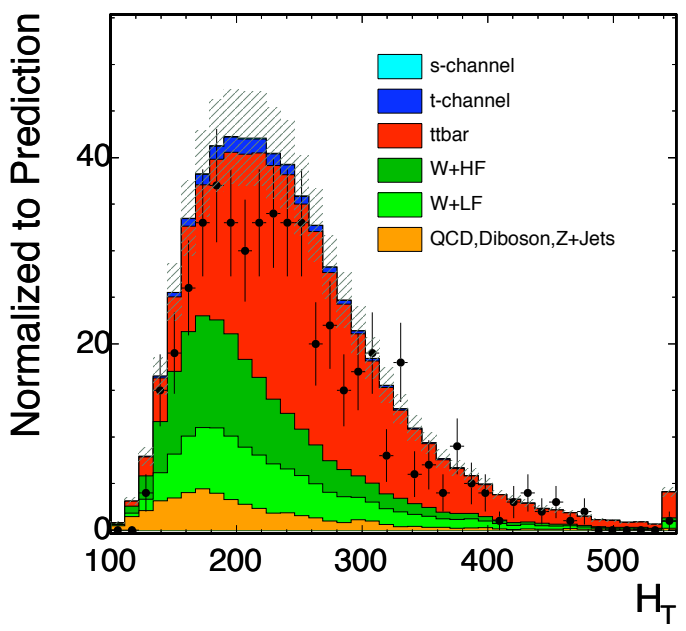

Figure 7.14: Stacked histogram of $H_{T}$ used as input variables to the $t$-channel 2-jet bin likelihood function. Data is compared to the prediction with each component normalized to its predicted value, shown in the 2-jet tag region (above) and 3-jet tag region (below). The modeling for $H_{T}$ looks fine in both plots, and the KS test values shown in Table 7.1 are not suspiciously low for this variable. 
CDF Run II Preliminary, $2.2 \mathrm{fb}^{-1}$

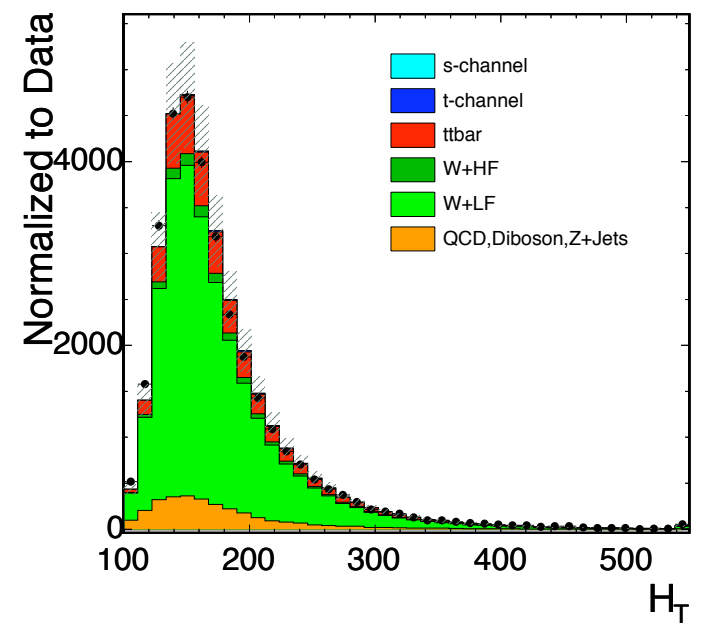

CDF Run II Preliminary, $2.2 \mathrm{fb}^{-1}$

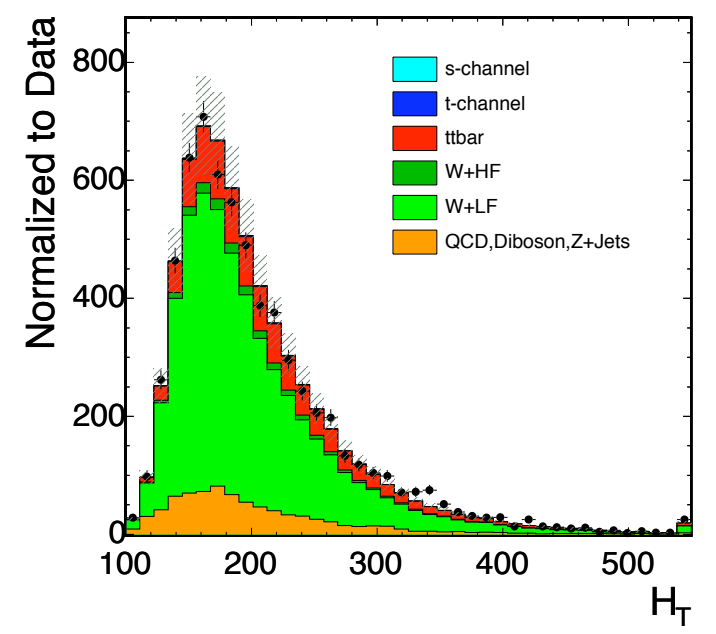

Figure 7.15: Stacked histogram of $H_{T}$ used as input variables to the $t$-channel 2-jet bin likelihood function. Data is compared to the prediction with each component normalized to its predicted value, shown in the 2-jet taggable region (above) and 3-jet taggable region (below). The modeling for $H_{T}$ looks fine in both plots. 

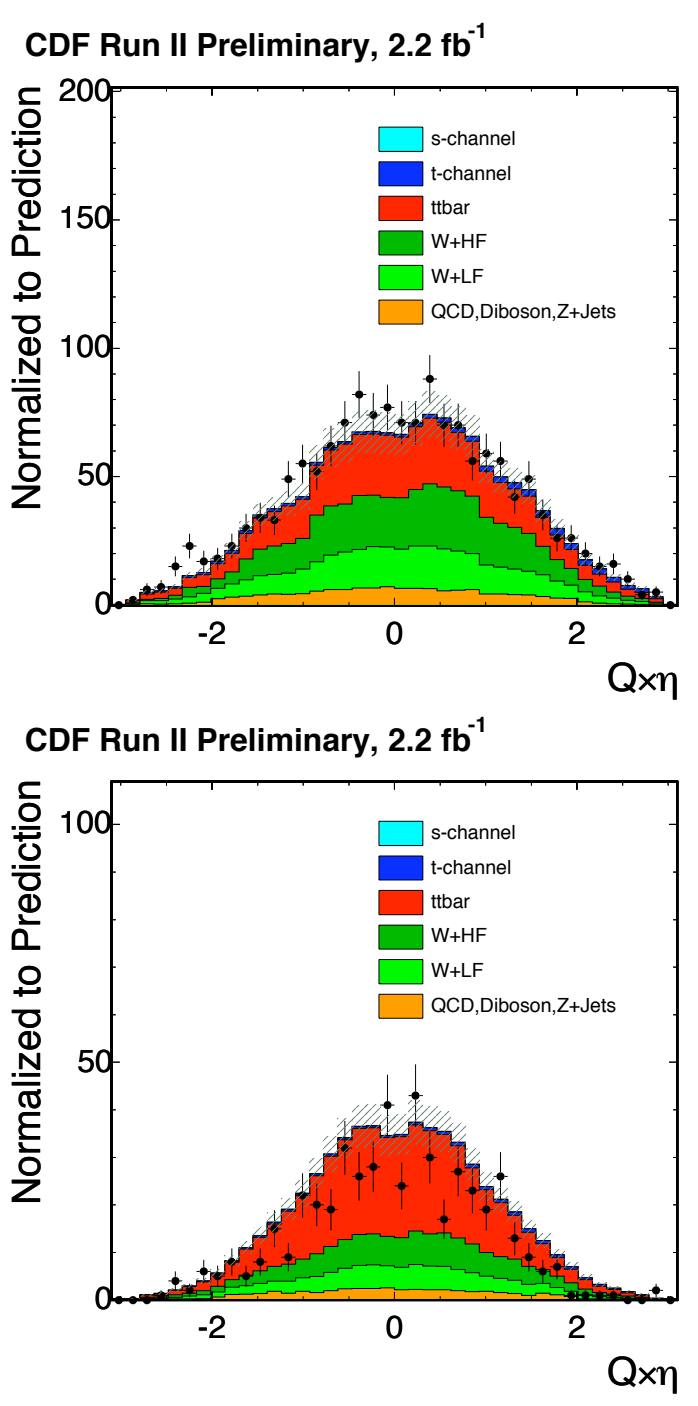

Figure 7.16: Stacked histogram of $Q \times \eta$ used as input variables to the $t$-channel 2-jet bin likelihood function. Data is compared to the prediction with each component normalized to its predicted value, shown in the 2-jet tag region (above) and 3-jet tag region (below). The mis-modeling of this plot in the high $-Q \times \eta$ - region is addressed in the modeling systematic on the $\eta$ of the lowest momentum jet. 

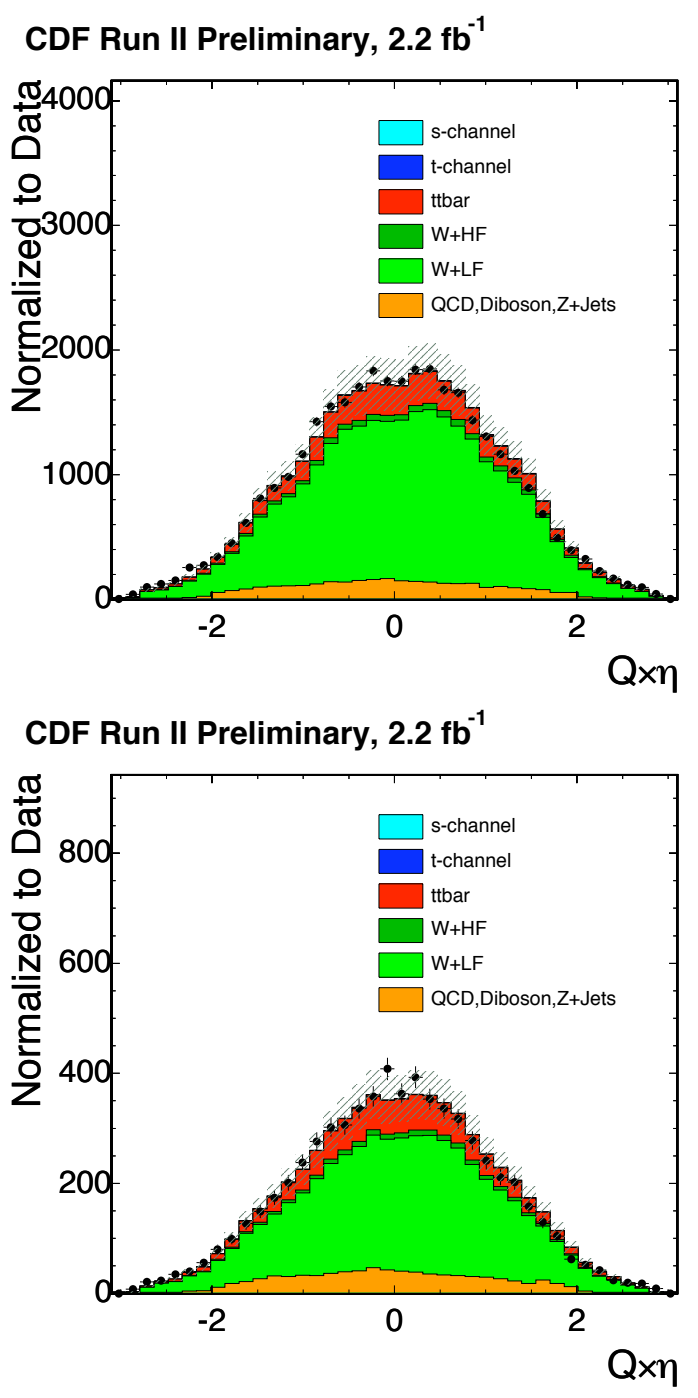

Figure 7.17: Stacked histogram of $Q \times \eta$ used as input variables to the $t$-channel 2-jet bin likelihood function. Data is compared to the prediction with each component normalized to its predicted value, shown in the 2 -jet taggable region (above) and 3-jet taggable region (below). 


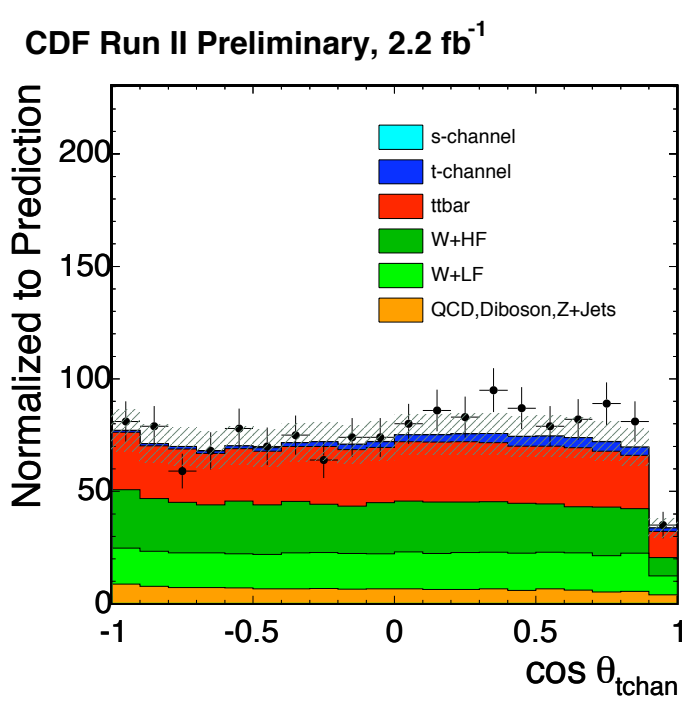

CDF Run II Preliminary, $2.2 \mathrm{fb}^{-1}$

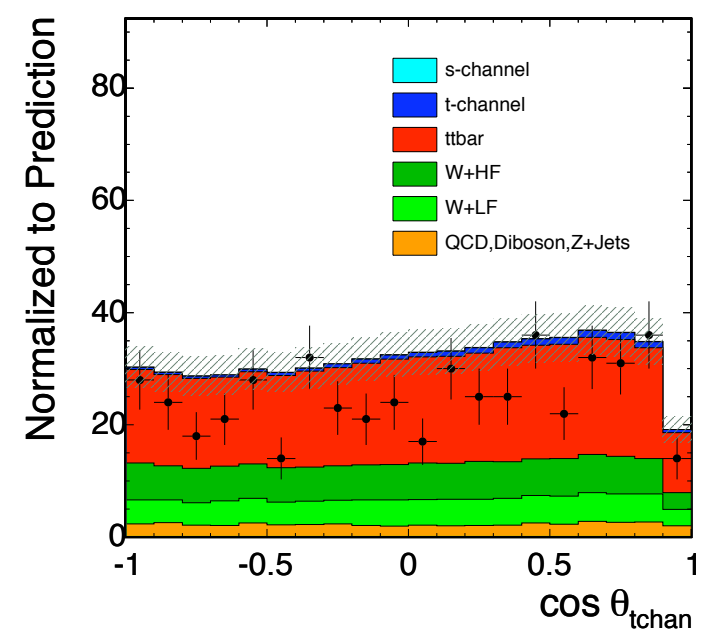

Figure 7.18: Stacked histogram of $\cos \theta_{t-\text { chan }}$ used as input variables to the $t$ channel 2-jet bin likelihood function. Data is compared to the prediction with each component normalized to its predicted value, shown in the 2-jet tag region (above) and 3-jet tag region (below). The modeling for $\cos \theta_{t-\text { chan }}$ looks fine in both plots, and the KS test values shown in Table 7.1 are slightly low, but only in the 2-tag region. The taggable plots in Figure 7.19 look fine. 


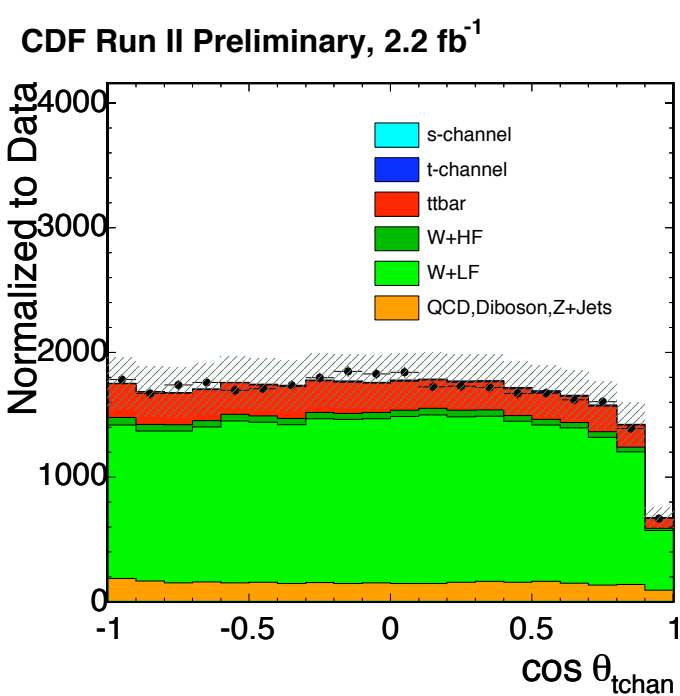

CDF Run II Preliminary, $2.2 \mathrm{fb}^{-1}$

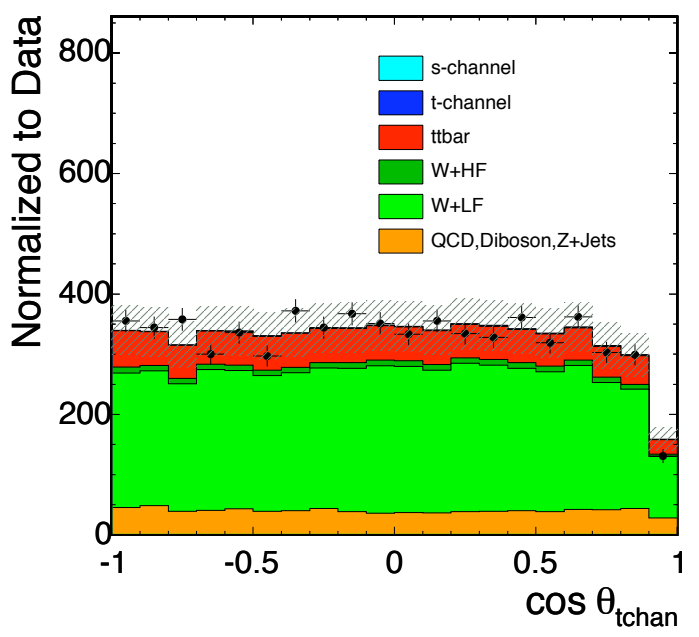

Figure 7.19: Stacked histogram of $\cos \theta_{t-\text { chan }}$ used as input variables to the $t$ channel 2-jet bin likelihood function. Data is compared to the prediction with each component normalized to its predicted value, shown in the 2-jet taggable region (above) and 3 -jet taggable region (below). The modeling for $\cos \theta_{t-\text { chan }}$ looks fine in both plots. 

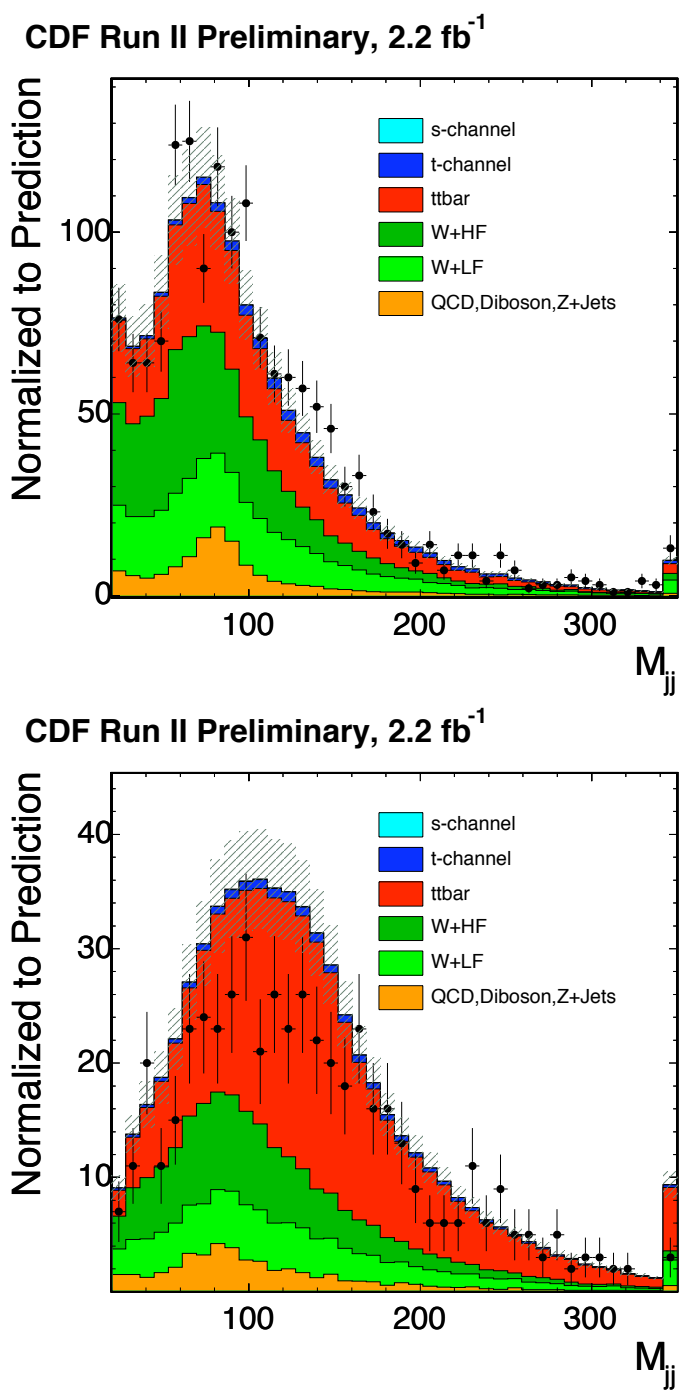

Figure 7.20: Stacked histogram of $M_{j j}$ used as input variables to the $t$-channel 2-jet bin likelihood function. Data is compared to the prediction with each component normalized to its predicted value, shown in the 2-jet tag region (above) and 3 -jet tag region (below). 


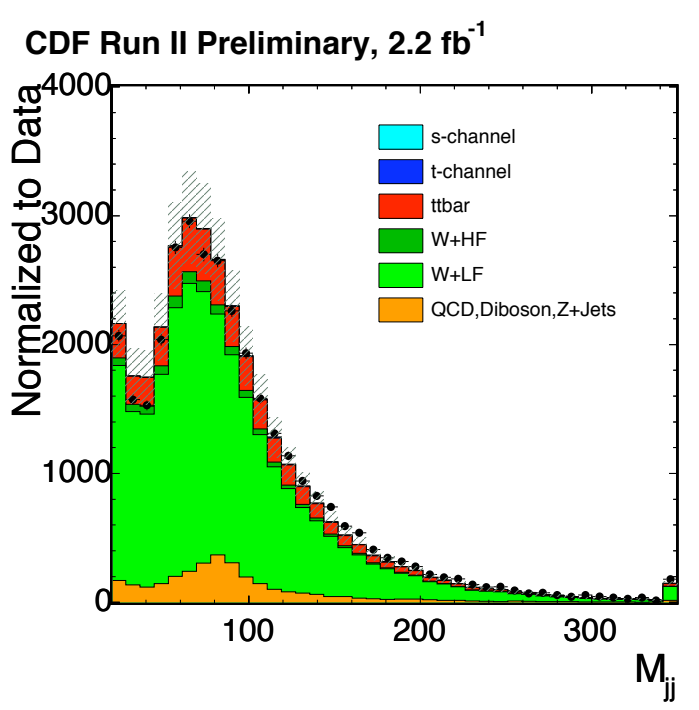

CDF Run II Preliminary, $2.2 \mathrm{fb}^{-1}$

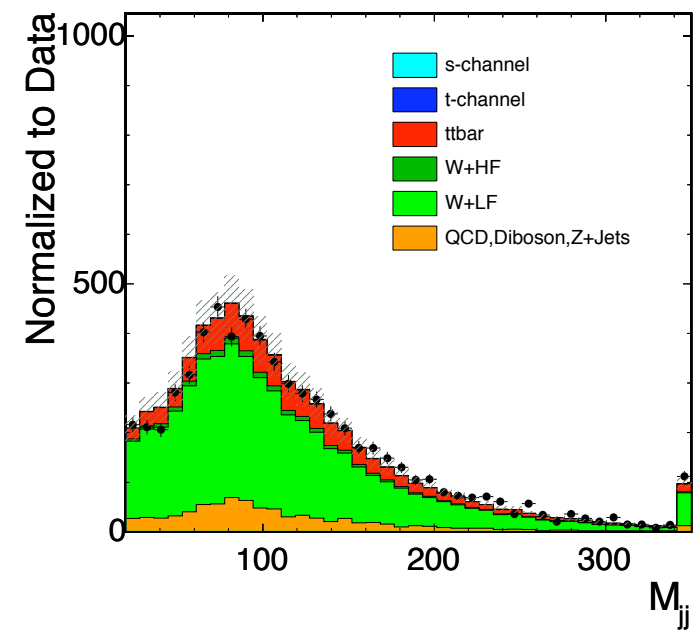

Figure 7.21: Stacked histogram of $M_{j j}$ used as input variables to the $t$-channel 2-jet bin likelihood function. Data is compared to the prediction with each component normalized to its predicted value, shown in the 2-jet taggable region (above) and 3-jet taggable region (below). The modeling for $M_{j j}$ looks fine in both plots. 

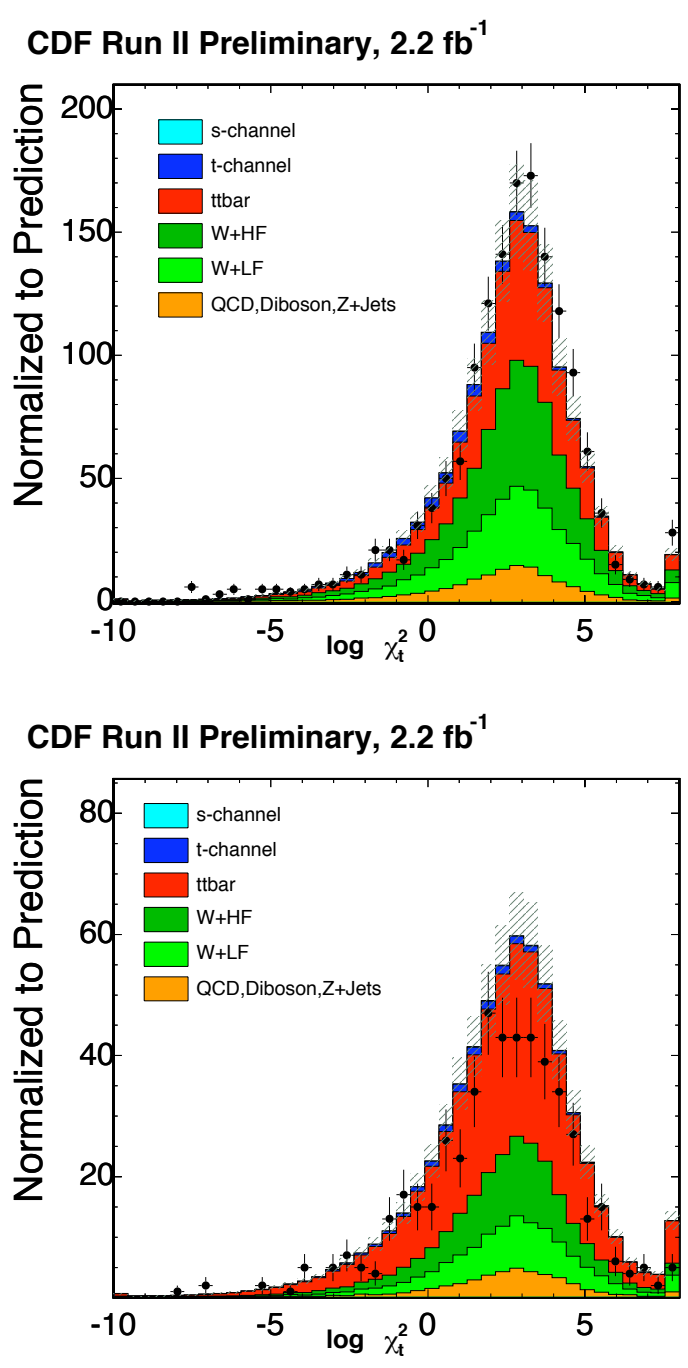

Figure 7.22: Stacked histogram of $\log \left(\chi_{t}^{2}\right)$ used as input variables to the $t$ channel 2-jet bin likelihood function. Data is compared to the prediction with each component normalized to its predicted value, shown in the 2-jet tag region (above) and 3-jet tag region (below). 


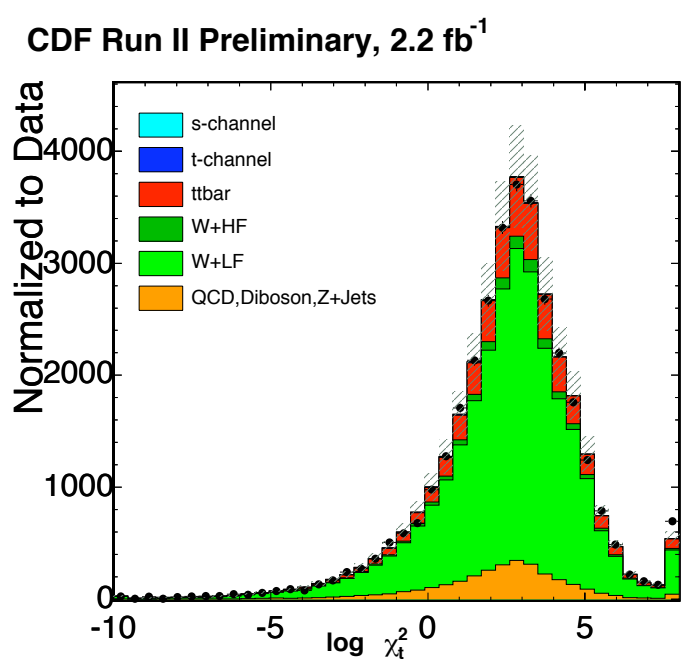

CDF Run II Preliminary, $2.2 \mathrm{fb}^{-1}$

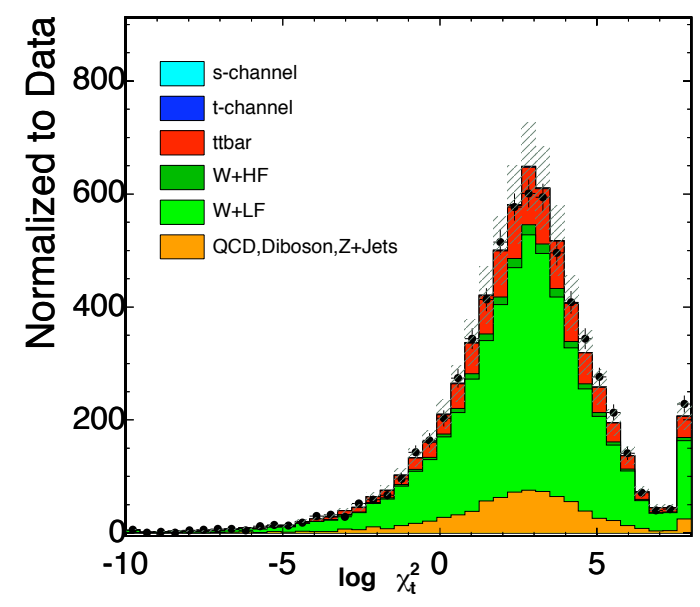

Figure 7.23: Stacked histogram of $\log \left(\chi_{t}^{2}\right)$ used as input variables to the $t$ channel 2-jet bin likelihood function. Data is compared to the prediction with each component normalized to its predicted value, shown in the 2-jet taggable region (above) and 3-jet taggable region (below). The modeling for $\log \left(\chi_{t}^{2}\right)$ looks fine in both plots. 


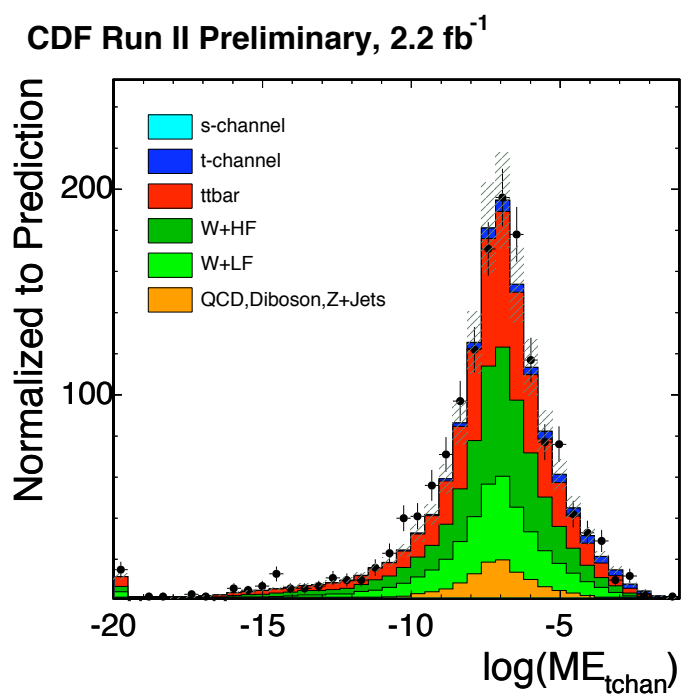

CDF Run II Preliminary, $2.2 \mathrm{fb}^{-1}$

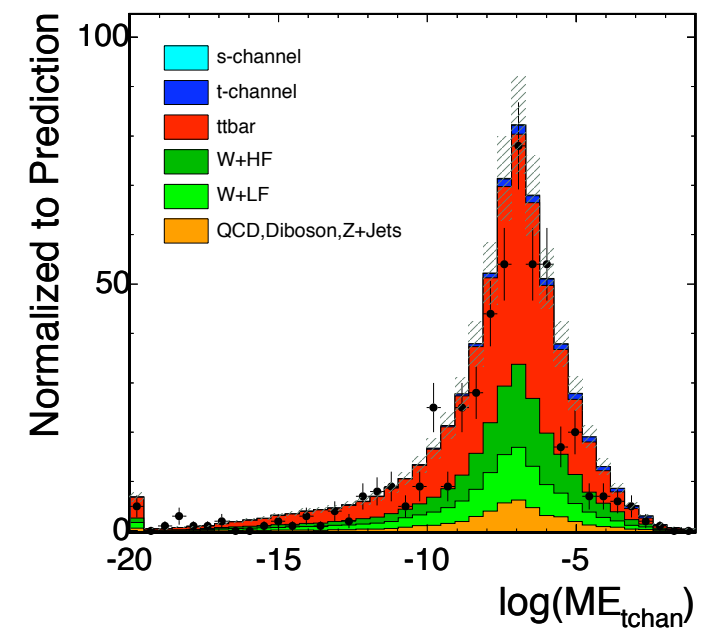

Figure 7.24: Stacked histogram of $\log \left(\mathrm{ME}_{t-\mathrm{chan}}\right)$ used as input variables to the $t$-channel 2-jet bin likelihood function. Data is compared to the prediction with each component normalized to its predicted value, shown in the 2-jet tag region (above) and 3-jet tag region (below). 


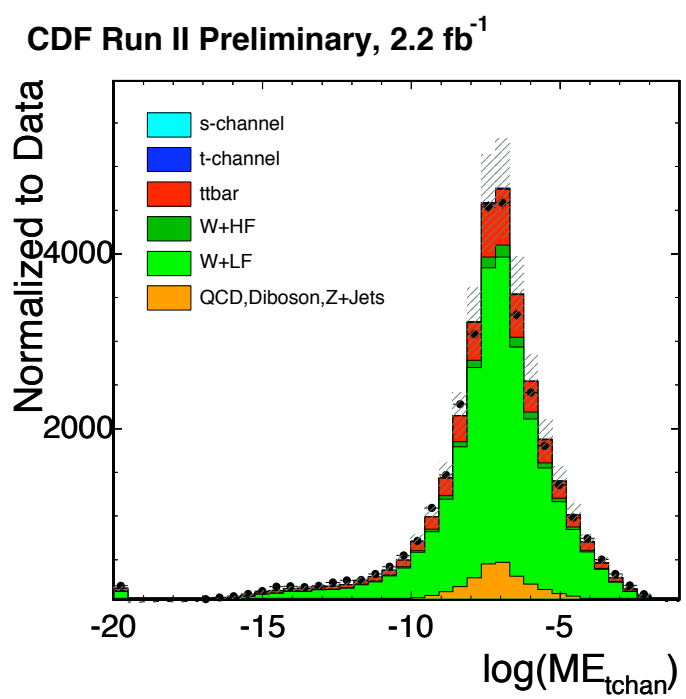

CDF Run II Preliminary, $2.2 \mathrm{fb}^{-1}$

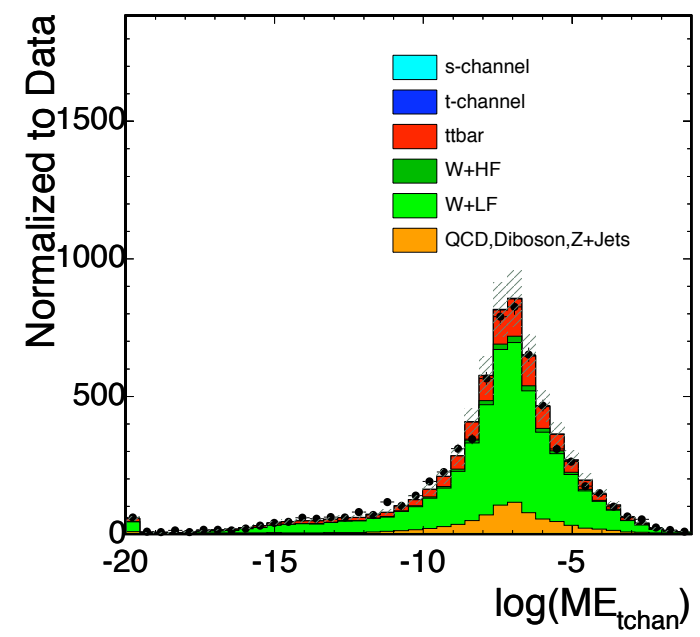

Figure 7.25: Stacked histogram of $\log \left(\mathrm{ME}_{t-\text { chan }}\right)$ used as input variables to the $t$-channel 2-jet bin likelihood function. Data is compared to the prediction with each component normalized to its predicted value, shown in the 2-jet taggable region (above) and 3-jet taggable region (below). The modeling for $\log \left(\mathrm{ME}_{t-\text { chan }}\right)$ looks fine in both plots. 


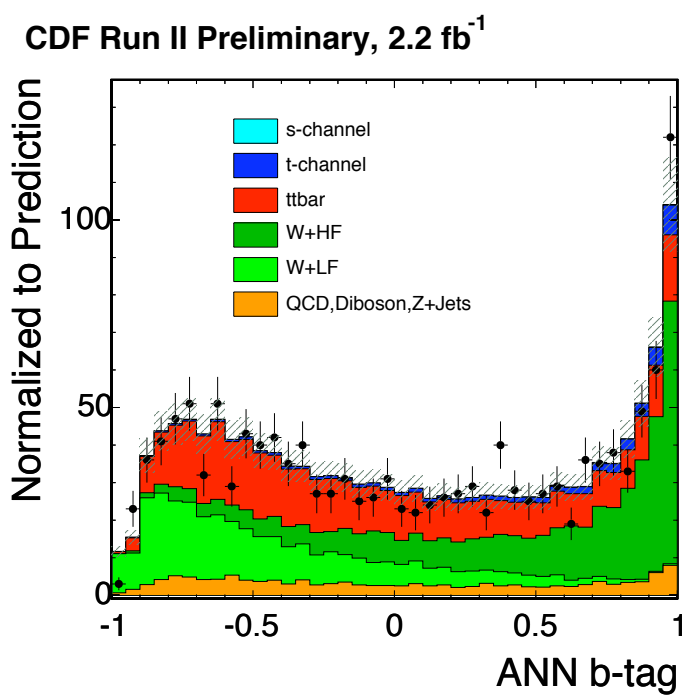

CDF Run II Preliminary, $2.2 \mathrm{fb}^{-1}$

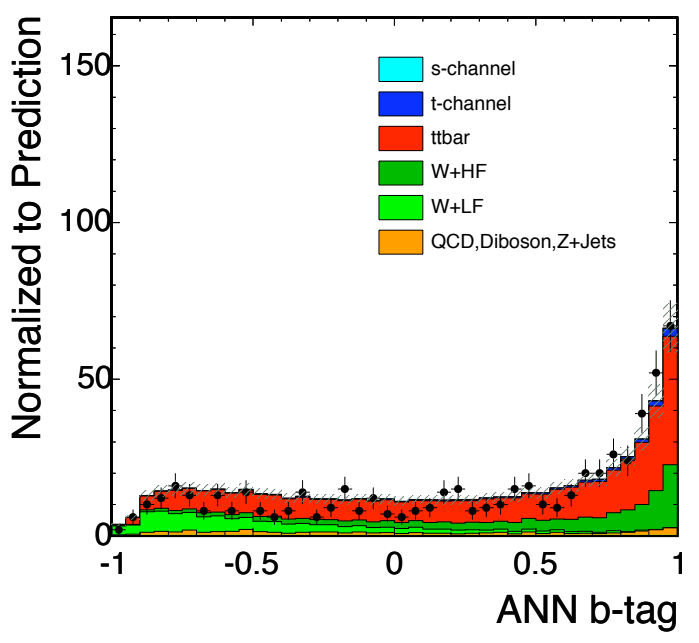

Figure 7.26: Stacked histogram of ANN b-tag used as input variables to the $t$-channel 2-jet bin likelihood function. Data is compared to the prediction with each component normalized to its predicted value, shown in the 2-jet tag region (above) and 3-jet tag region (below). The modeling for ANN $b$-tag looks fine in both plots, although the KS test is slightly low in the 3-jet bin in Table 7.1.Although no modeling systematic is taken based on this plot, there is an overall shape systematic for the ANN $b$-tag distribution derived from the ANN $b$-tag correction function, as described in Section 6.3. No plot is made of this variable in the taggable region, as it is only defined for jets with SECVTX tags. 


\subsection{2 $\quad \mathcal{L}_{3 t}$ Input Variables}

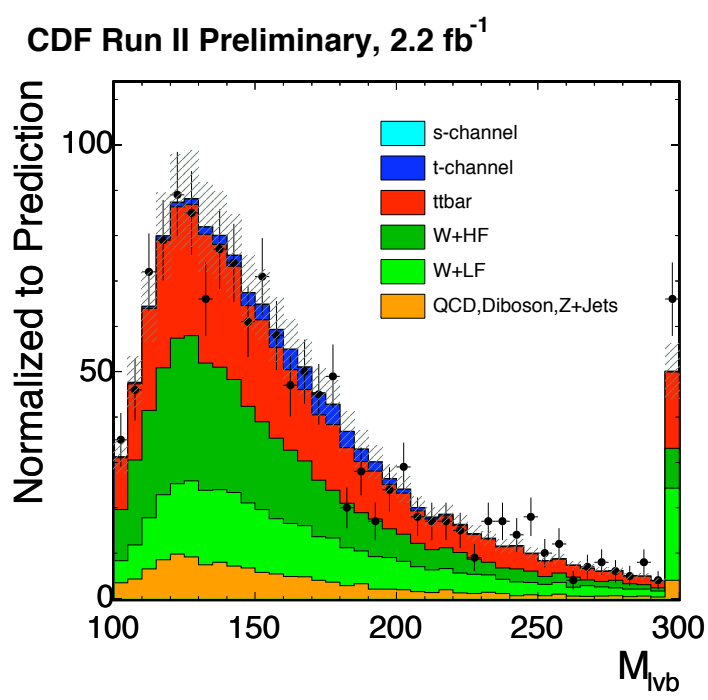

CDF Run II Preliminary, $2.2 \mathrm{fb}^{-1}$

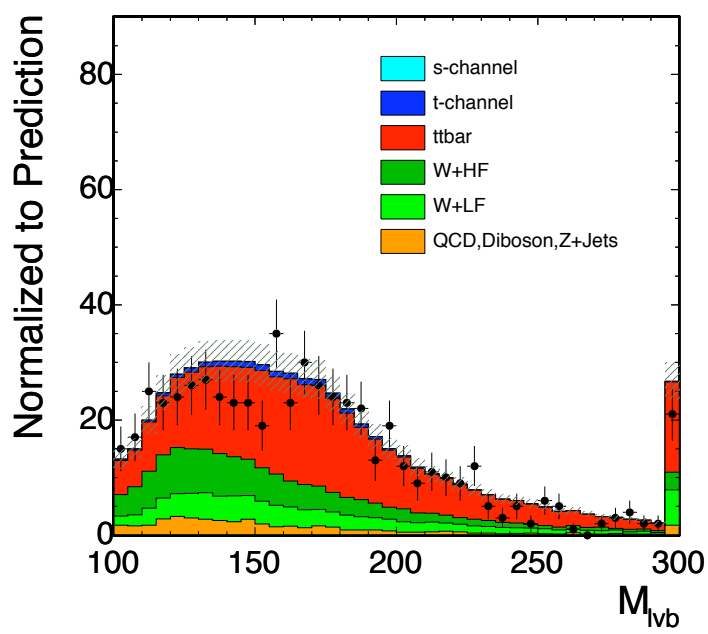

Figure 7.27: Stacked histogram of $m_{\ell \nu b}$ used as input variables to the $t$-channel 3 -jet bin likelihood function. Data is compared to the prediction with each component normalized to its predicted value, shown in the 2-jet tag region (above) and 3-jet tag region (below). The modeling for $m_{\ell \nu b}$ looks fine in both plots, and the KS test values shown in Table 7.1 are fine for this variable, especially in the 3 -jet bin where this variable is used. 

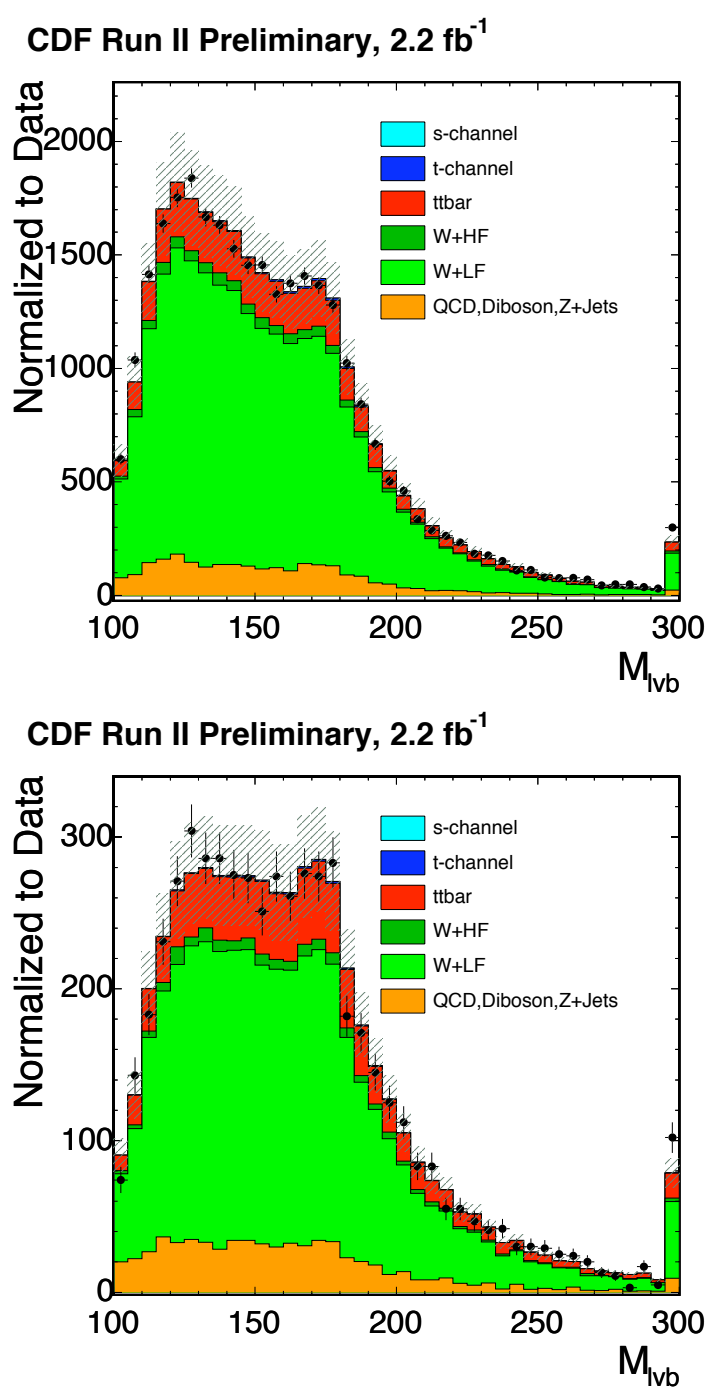

Figure 7.28: Stacked histogram of $m_{\ell \nu b}$ used as input variables to the $t$-channel 3-jet bin likelihood function. Data is compared to the prediction with each component normalized to its predicted value, shown in the 2-jet taggable region (above) and 3-jet taggable region (below). The modeling for $m_{\ell \nu b}$ looks fine in both plots. 

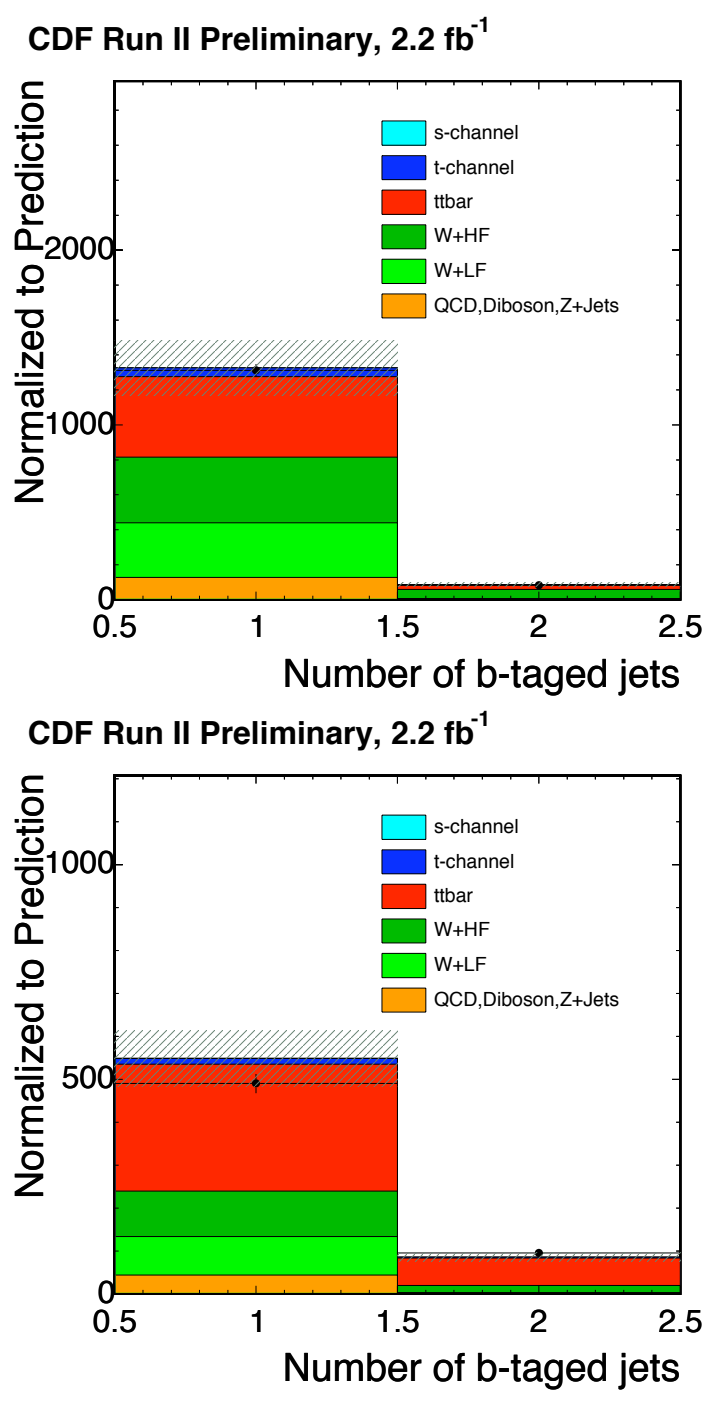

Figure 7.29: Stacked histogram of Number of tags used as input variables to the $t$-channel 3-jet bin likelihood function. Data is compared to the prediction with each component normalized to its predicted value, shown in the 2-jet tag region (above) and 3-jet tag region (below). The plot is not made in the taggable region as it requires SECVTX tags. 


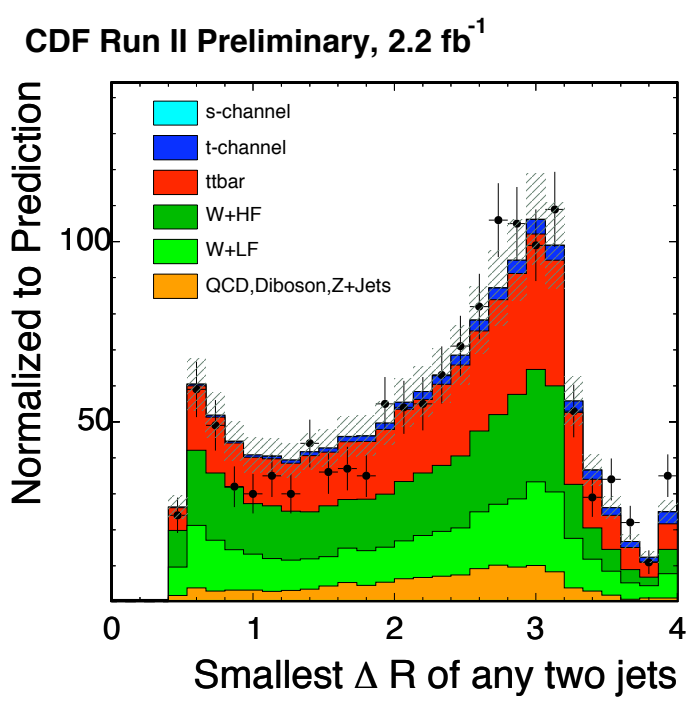

CDF Run II Preliminary, $2.2 \mathrm{fb}^{-1}$

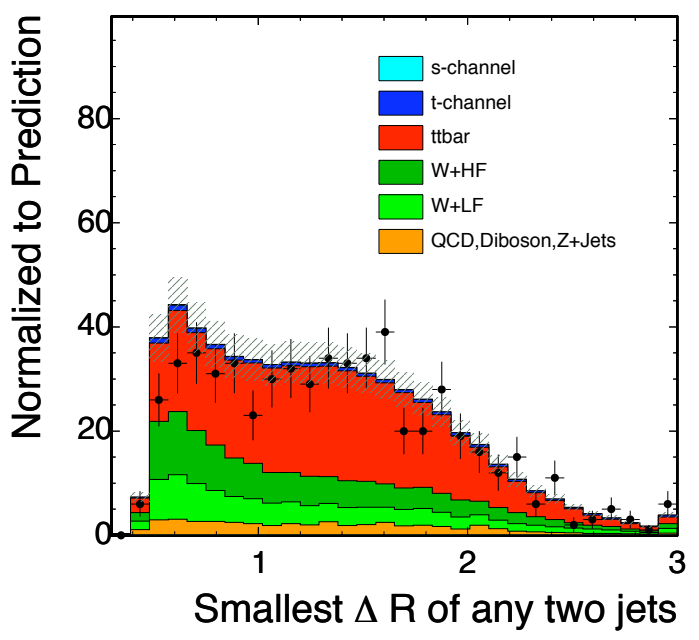

Figure 7.30: Stacked histogram of smallest $\Delta R$ of any two jets used as input variables to the $t$-channel 3 -jet bin likelihood function. Data is compared to the prediction with each component normalized to its predicted value, shown in the 2-jet tag region (above) and 3-jet tag region (below). This variable exhibits mismodeling, and has a very low KS value in the 2-tag region in Table 7.1. Since the 2-jet taggable plot is also mis-modeled, in Figure 7.31, a shape systematic was taken for this distribution in the 2-jet region. 

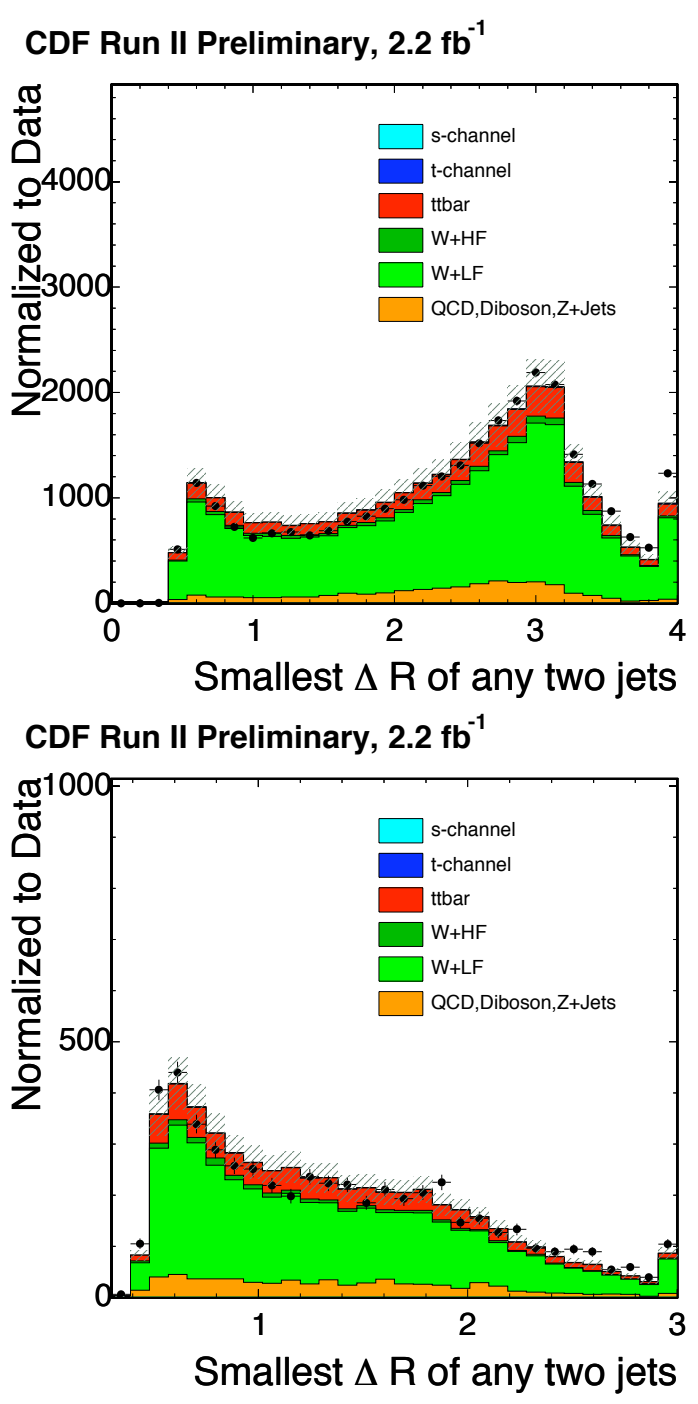

Figure 7.31: Stacked histogram of smallest $\Delta R$ of any two jets used as input variables to the $t$-channel 3 -jet bin likelihood function. Data is compared to the prediction with each component normalized to its predicted value, shown in the 2-jet taggable region (above) and 3-jet taggable region (below). The modeling for the smallest $\Delta R$ exhibits mis-modeling in the 2-jet taggable region, so a shape systematic was taken for this distribution in the 2 -jet region. The modeling in the 3 -jet taggable plot does not look so severely mis-modeled, so no systematic was taken. 


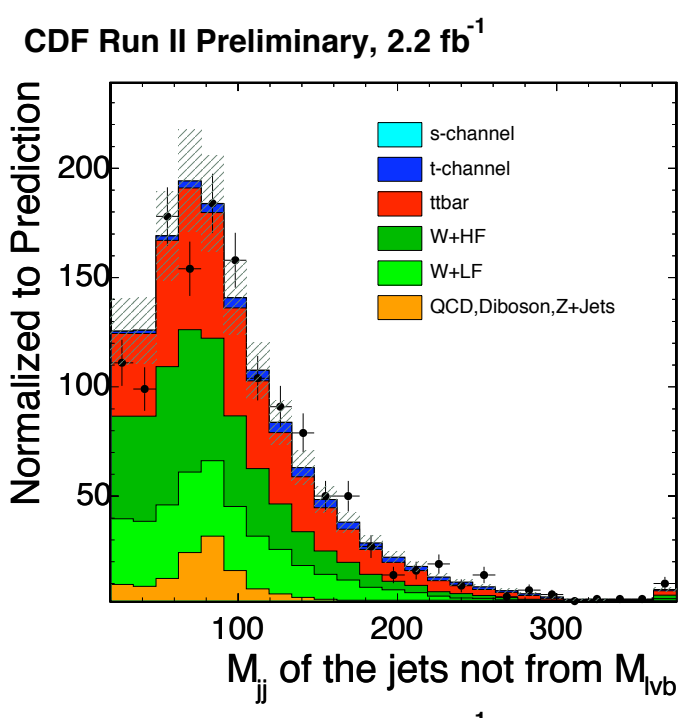

CDF Run II Preliminary, $2.2 \mathrm{fb}^{-1}$

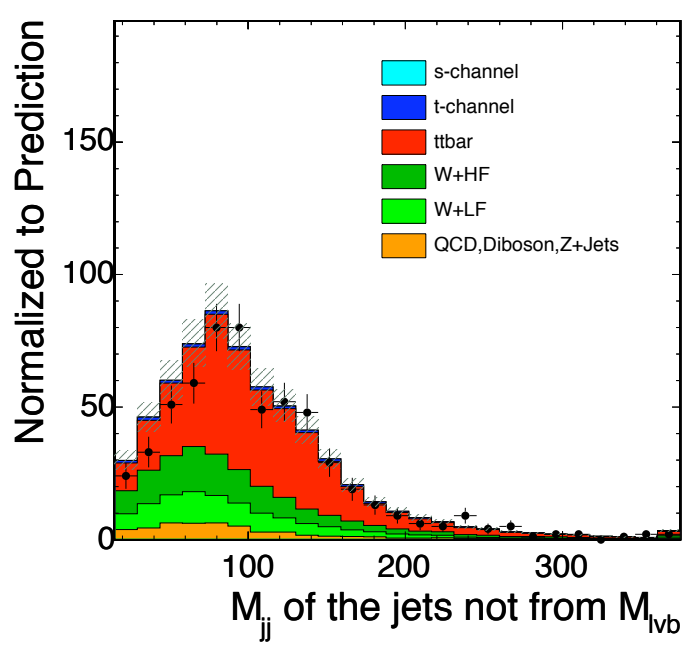

Figure 7.32: Stacked histogram of $M_{j j}$ of the two jets not chosen to be the $b$ from top decay used as input variables to the $t$-channel 3 -jet bin likelihood function. Data is compared to the prediction with each component normalized to its predicted value, shown in the 2-jet tag region (above) and 3-jet tag region (below). The KS test for $M_{j j}$ is a bit low, and this possible mis-modeling was explored for a very similar plot, $M_{j j}$ of the two leading jets, as explained in Section 7.4.1. 


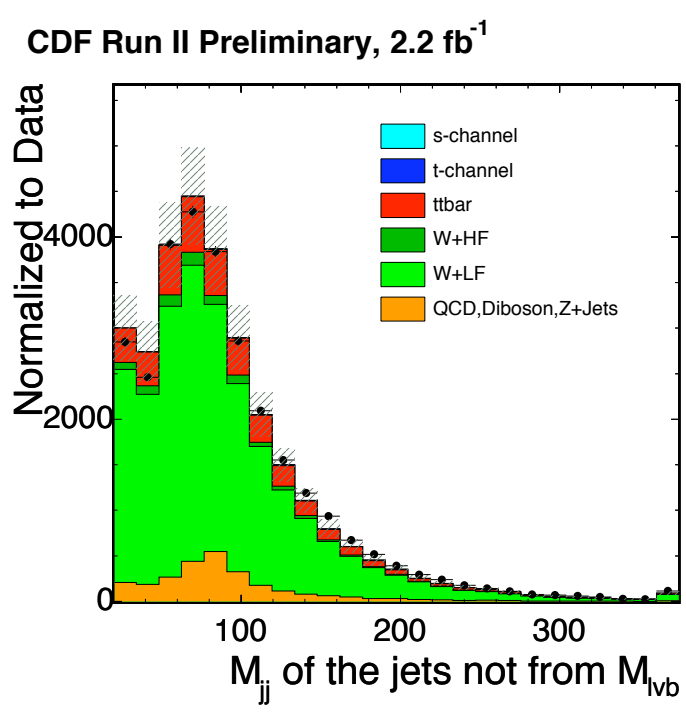

CDF Run II Preliminary, $2.2 \mathrm{fb}^{-1}$

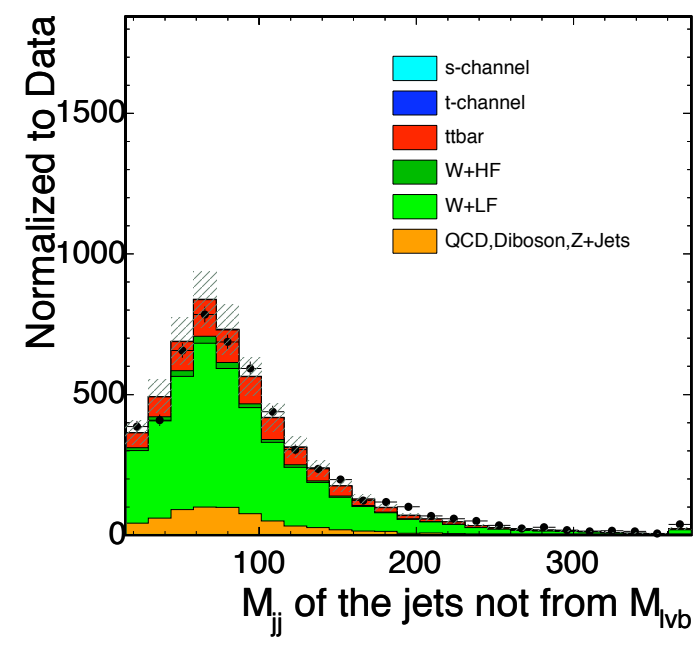

Figure 7.33: Stacked histogram of $M_{j j}$ of the two jets not chosen to be the $b$ from top decay used as input variables to the $t$-channel 3-jet bin likelihood function. Data is compared to the prediction with each component normalized to its predicted value, shown in the 2-jet taggable region (above) and 3-jet taggable region (below). The modeling for $M_{j j}$ of the two jets not chosen to be the $b$ from top decay looks fine in both plots. 


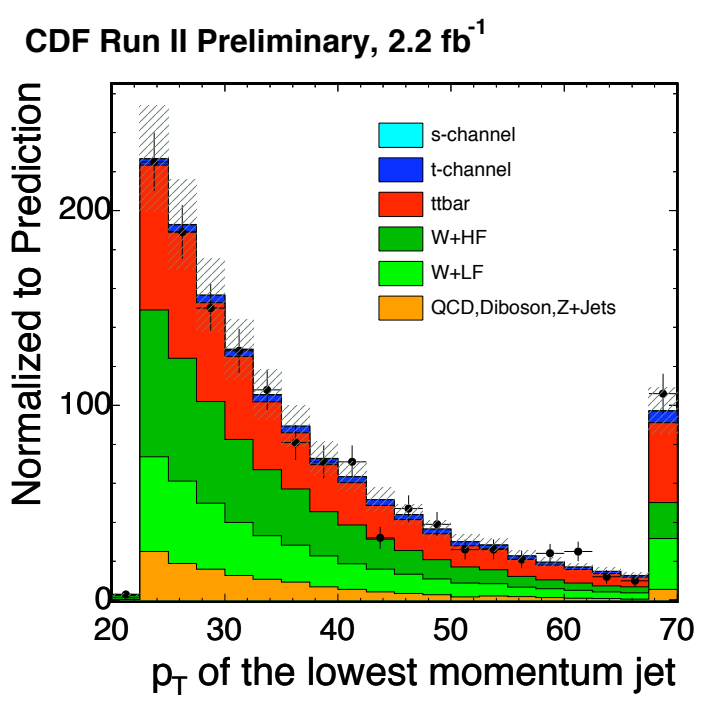

CDF Run II Preliminary, $2.2 \mathrm{fb}^{-1}$

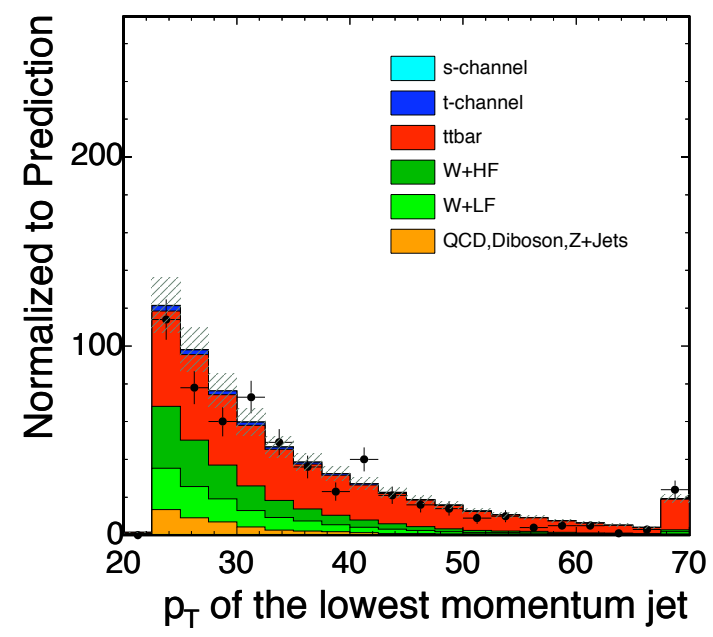

Figure 7.34: Stacked histogram of $P_{T}$ of the lowest momentum jet used as input variables to the $t$-channel 3 -jet bin likelihood function. Data is compared to the prediction with each component normalized to its predicted value, shown in the 2-jet tag region (above) and 3-jet tag region (below). The modeling for $P_{T}$ of the lowest momentum jet looks fine in both plots, and the KS test values shown in Table 7.1 are not suspiciously low for this variable. 

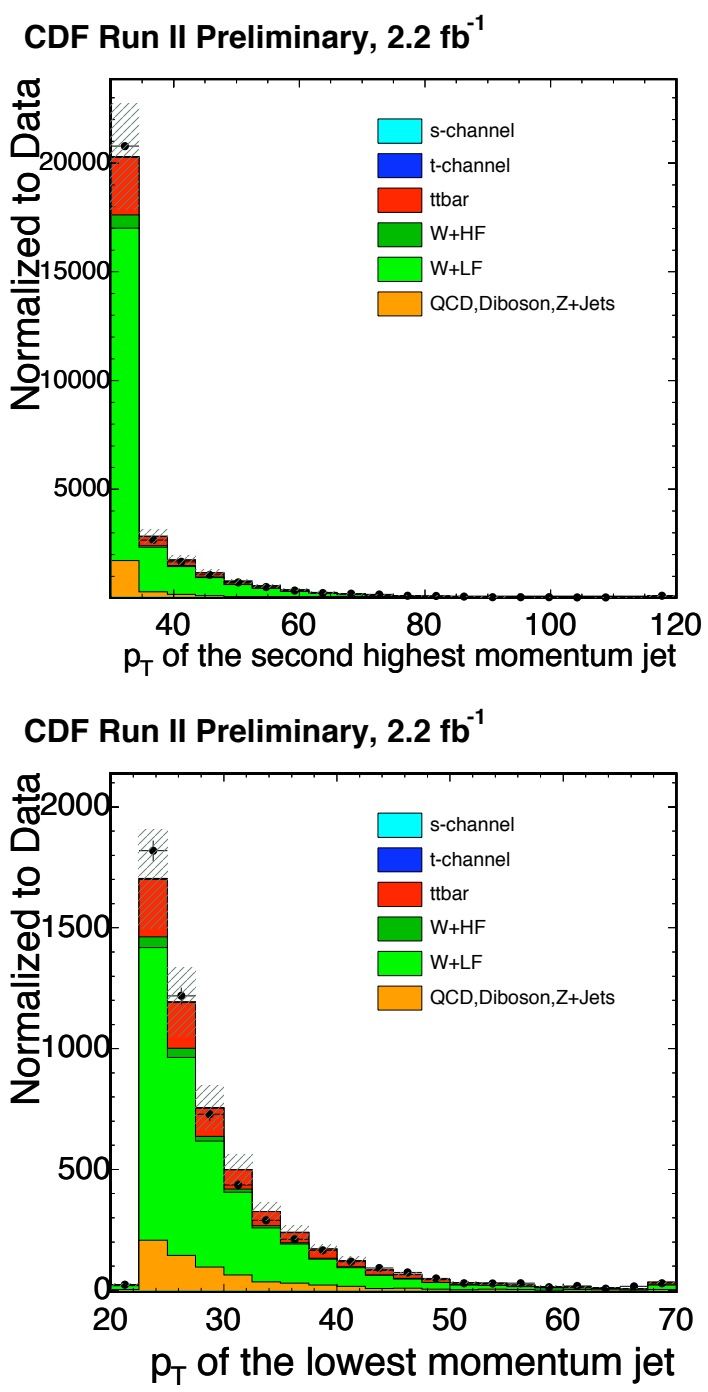

Figure 7.35: Stacked histogram of $P_{T}$ of the lowest momentum jet used as input variables to the $t$-channel 3 -jet bin likelihood function. Data is compared to the prediction with each component normalized to its predicted value, shown in the 2-jet taggable region (above) and 3-jet taggable region (below). The modeling for $P_{T}$ of the lowest momentum jet looks fine in both plots. 


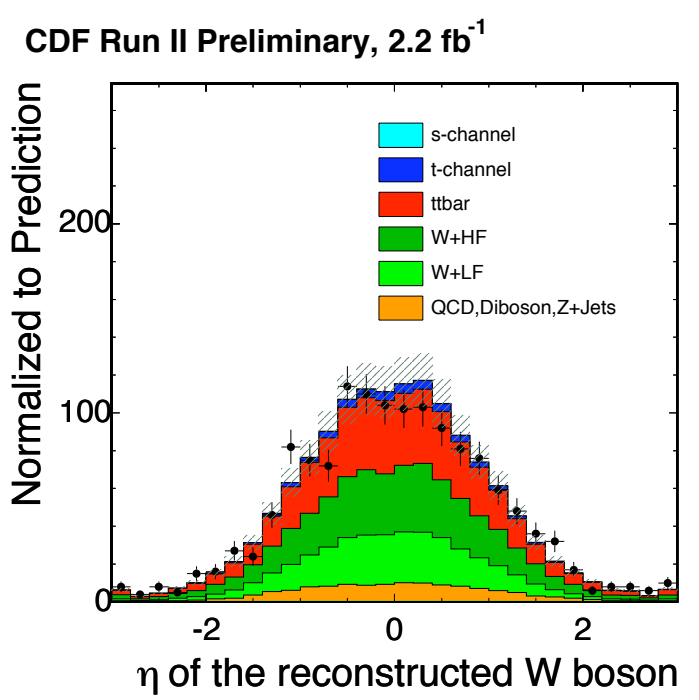

CDF Run II Preliminary, $2.2 \mathrm{fb}^{-1}$

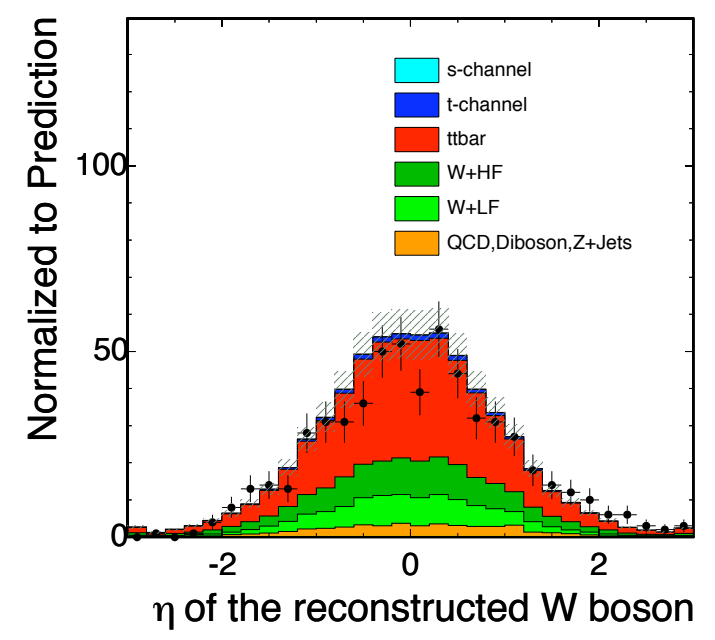

Figure 7.36: Stacked histogram of $\eta$ of the reconstructed $W$ boson used as input variables to the $t$-channel 3 -jet bin likelihood function. Data is compared to the prediction with each component normalized to its predicted value, shown in the 2-jet tag region (above) and 3-jet tag region (below). The modeling for $\eta$ of the reconstructed $W$ boson looks fine in both plots, and the KS test values shown in Table 7.1 are not suspiciously low for this variable. 

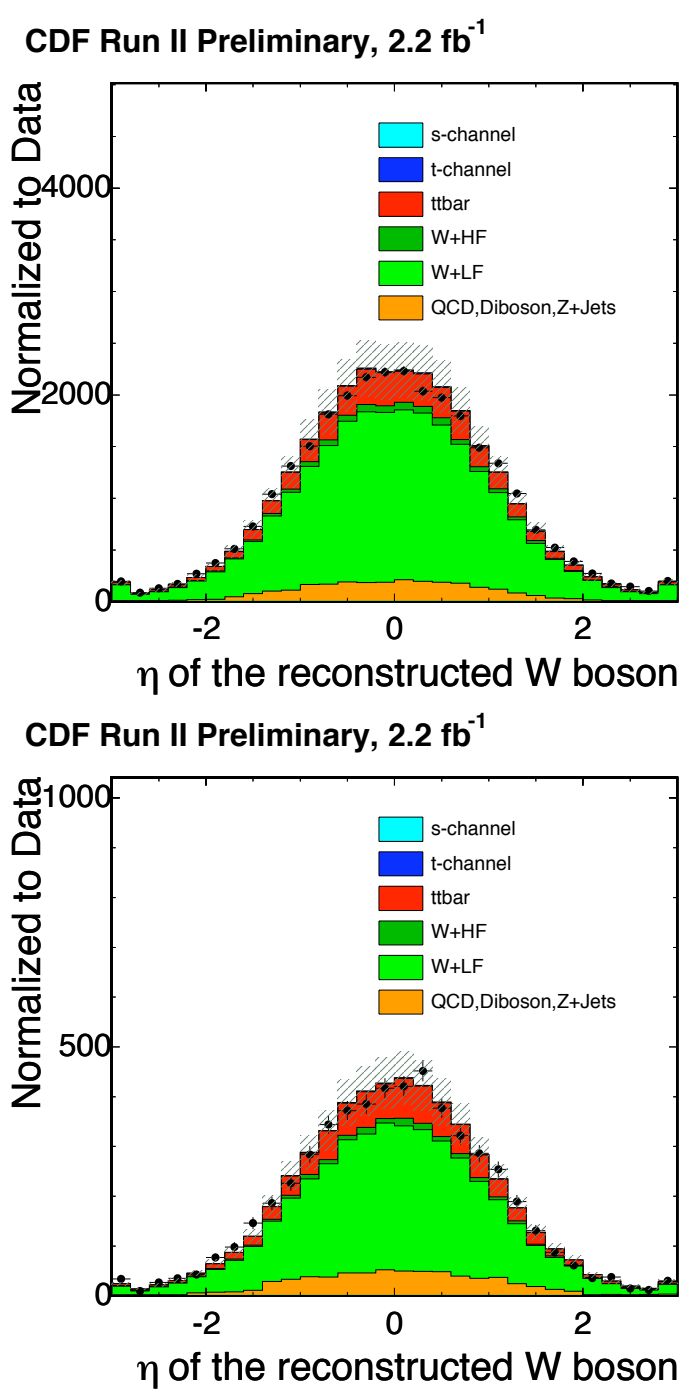

Figure 7.37: Stacked histogram of $\eta$ of the reconstructed $W$ boson used as input variables to the $t$-channel 3 -jet bin likelihood function. Data is compared to the prediction with each component normalized to its predicted value, shown in the 2-jet taggable region (above) and 3-jet taggable region (below). The modeling for $\eta$ of the reconstructed $W$ boson looks fine in both plots. 


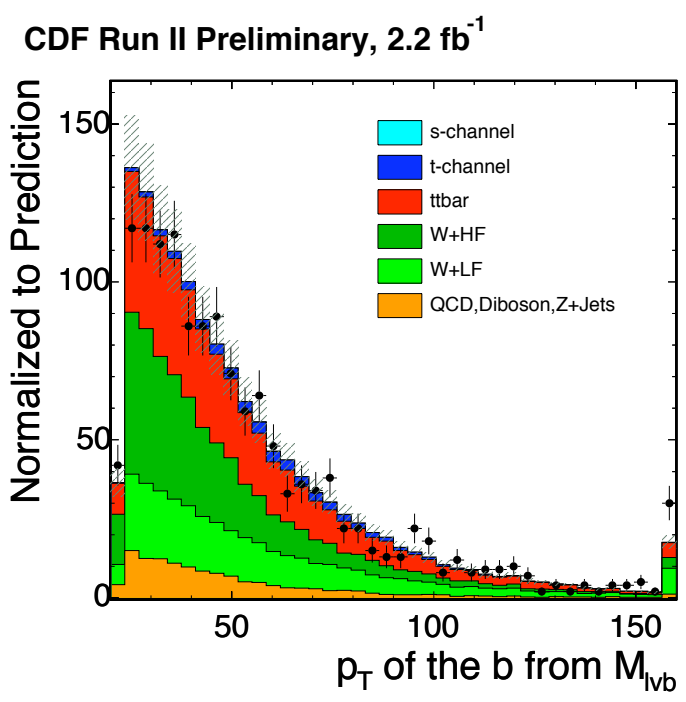

CDF Run II Preliminary, $2.2 \mathrm{fb}^{-1}$

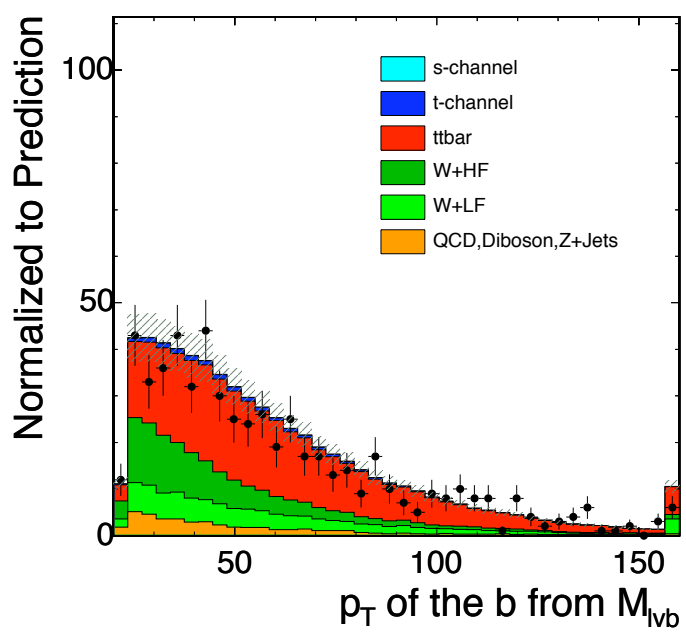

Figure 7.38: Stacked histogram of $p_{T}$ of the jet chosen to be the $b$ from top decay used as input variables to the $t$-channel 3 -jet bin likelihood function. Data is compared to the prediction with each component normalized to its predicted value, shown in the 2-jet tag region (above) and 3-jet tag region (below). The modeling for $p_{T}$ of the jet chosen to be the $b$ from top decay looks fine in both plots, and the KS test values shown in Table 7.1 are not suspiciously low for this variable. 
CDF Run II Preliminary, $2.2 \mathrm{fb}^{-1}$

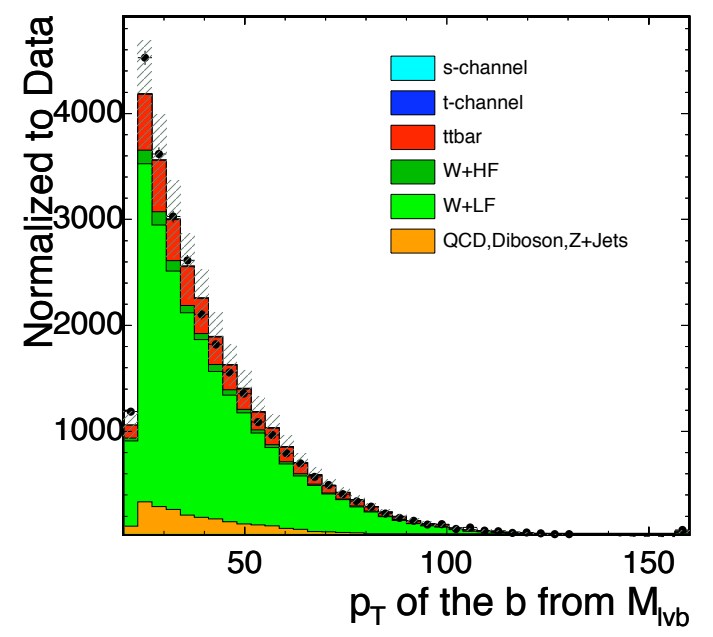

CDF Run II Preliminary, $2.2 \mathrm{fb}^{-1}$

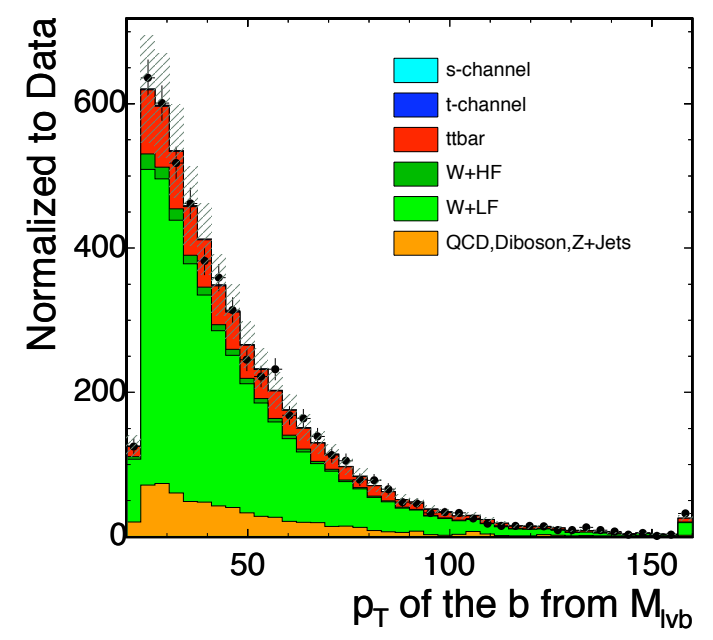

Figure 7.39: Stacked histogram of $p_{T}$ of the jet chosen to be the $b$ from top decay used as input variables to the $t$-channel 3 -jet bin likelihood function. Data is compared to the prediction with each component normalized to its predicted value, shown in the 2-jet taggable region (above) and 3-jet taggable region (below). The modeling for $p_{T}$ of the jet chosen to be the $b$ from top decay looks fine in both plots. 


\subsubsection{Further Kinematic Modeling Check Plots}
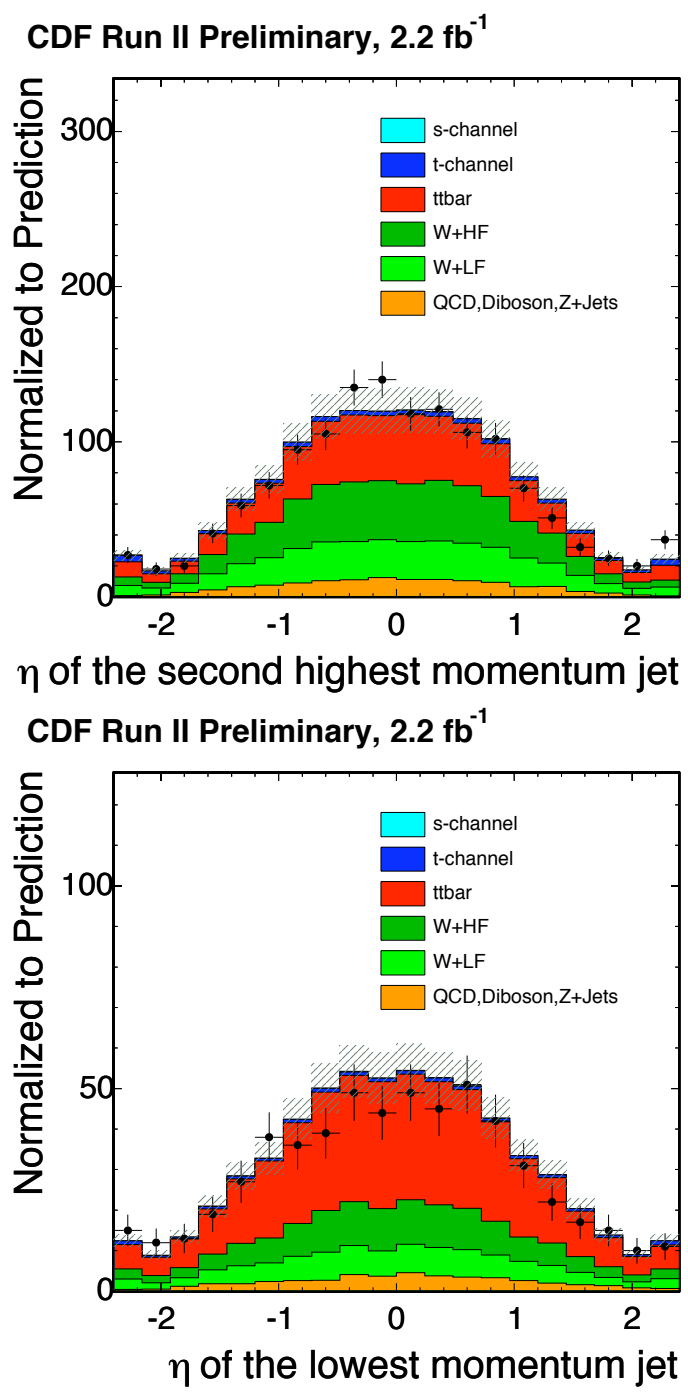

Figure 7.40: Stacked histogram of $\eta$ of the lowest momentum jet. Data is compared to the prediction with each component normalized to its predicted value, shown in the 2-jet tag region (above) and 3-jet tag region (below). Although the modeling of this plot is acceptable, the mis-modeling in the high $|\eta|$ region of the taggable plot (see 7.41) indicates the need for the modeling systematic taken for this distribution. 


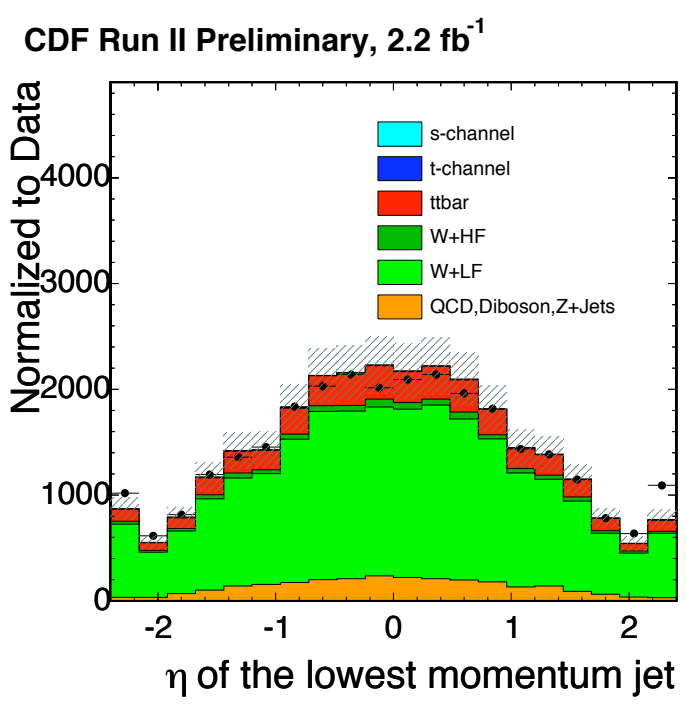

CDF Run II Preliminary, $2.2 \mathrm{fb}^{-1}$

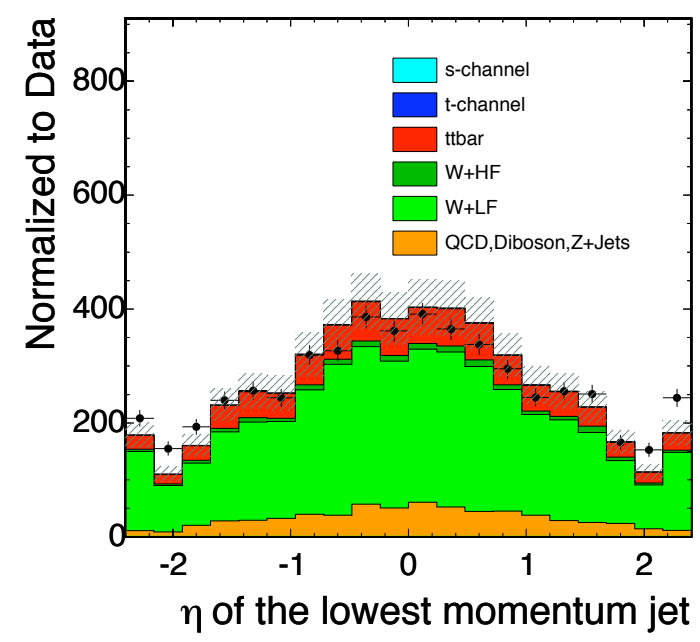

Figure 7.41: Stacked histogram of $\eta$ of the lowest momentum jet. Data is compared to the prediction with each component normalized to its predicted value, shown in the 2-jet taggable region (above) and 3-jet taggable region (below). The mis-modeling in the high $|\eta|$ region indicates the need for the modeling systematic taken for this distribution. 

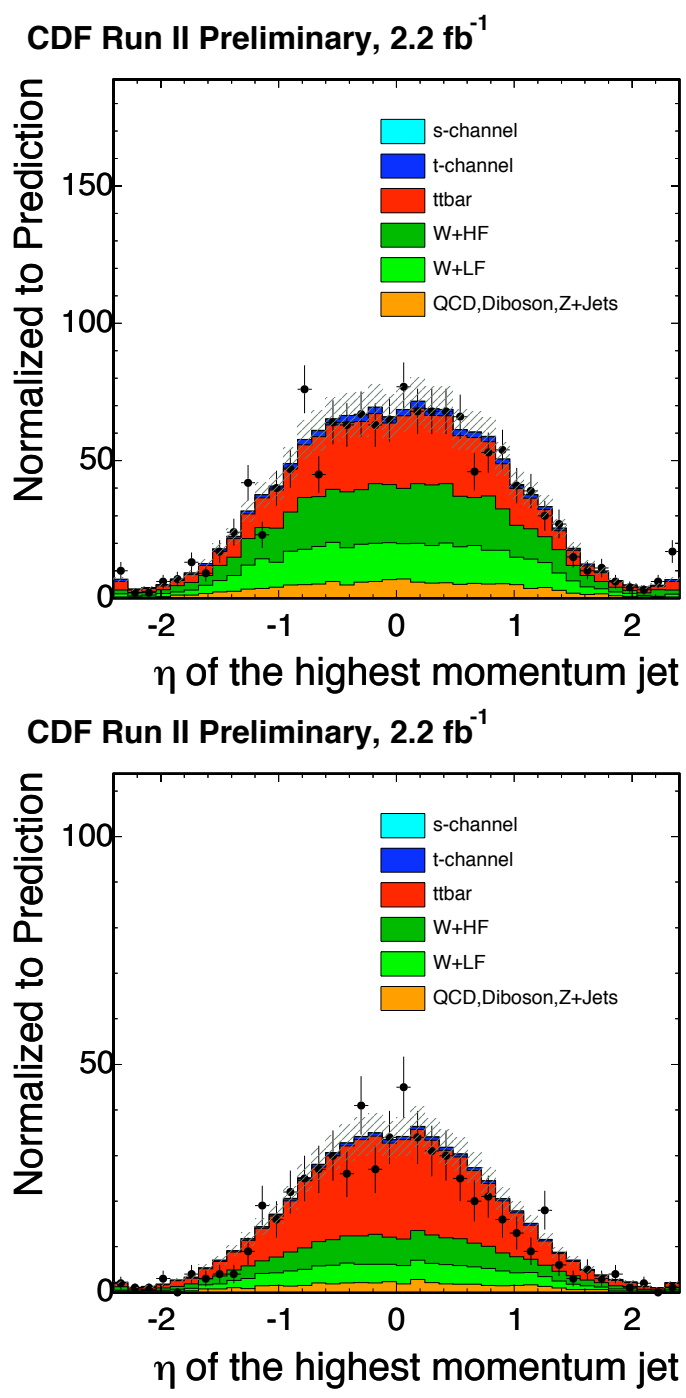

Figure 7.42: Stacked histogram of $\eta$ of the leading jet. Data is compared to the prediction with each component normalized to its predicted value, shown in the 2 -jet tag region (above) and 3 -jet tag region (below). 

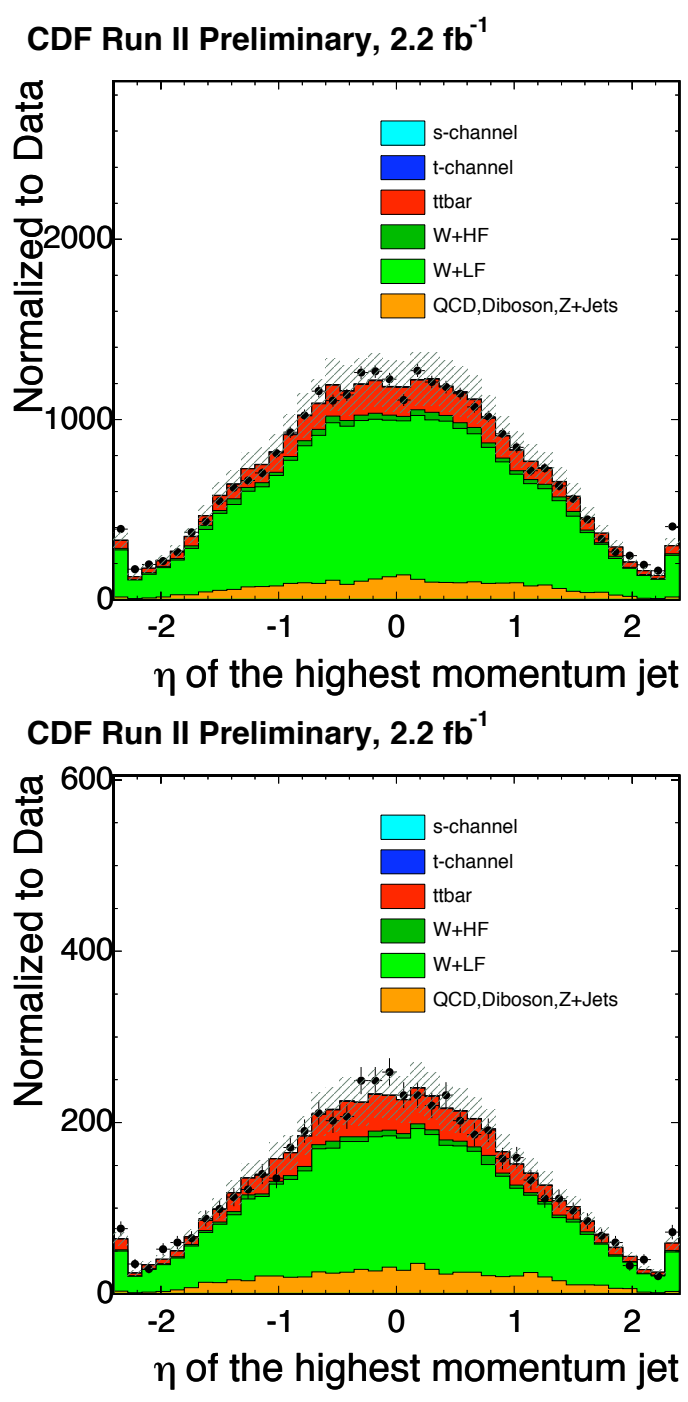

Figure 7.43: Stacked histogram of $\eta$ of the leading jet. Data is compared to the prediction with each component normalized to its predicted value, shown in the 2-jet taggable region (above) and 3-jet taggable region (below). Mis-modeling in the high $\eta$ region is likely related to the mis-modeling shown in Figure 7.41, but is less pronounced. 

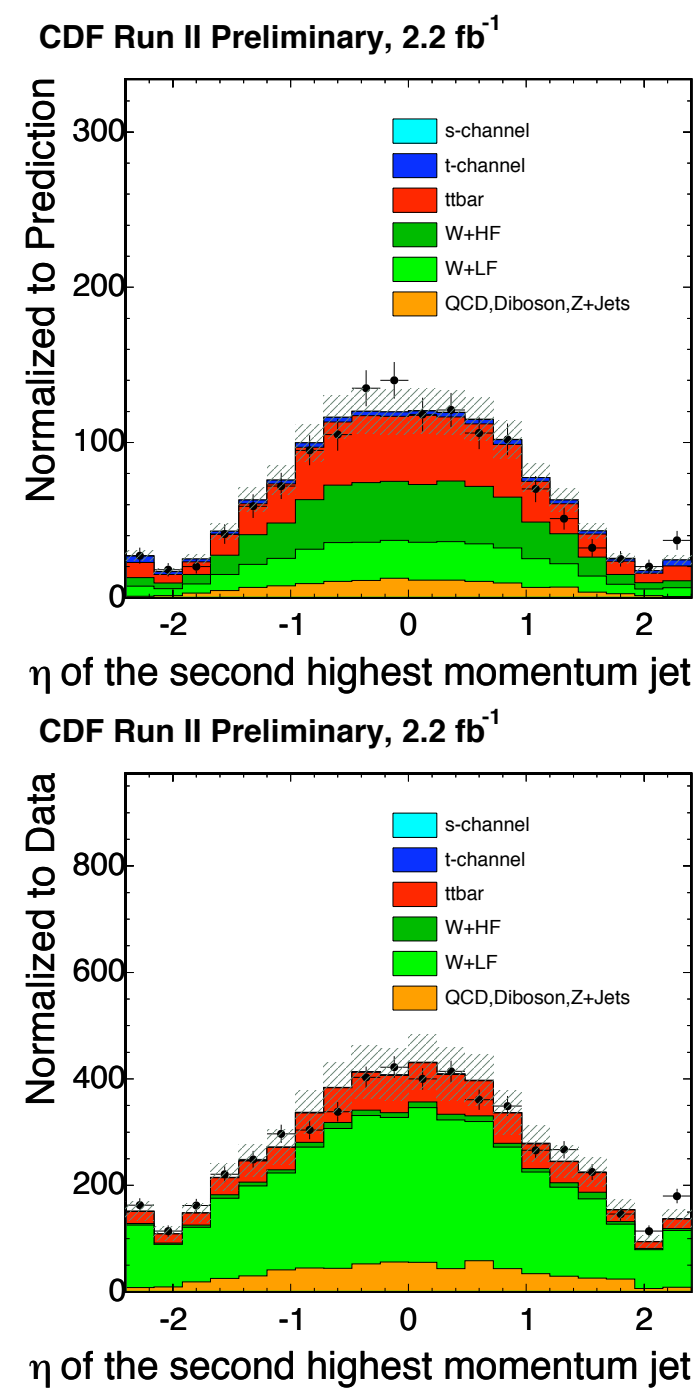

Figure 7.44: Stacked histogram of $\eta$ of the second jet. Data is compared to the prediction with each component normalized to its predicted value, shown in the 2 -jet tag region (above) and 3-jet tag region (below). For 2-jet plots, this is the same distribution as the $\eta$ of the lowest momentum jet, for which a modeling systematic is applied. 


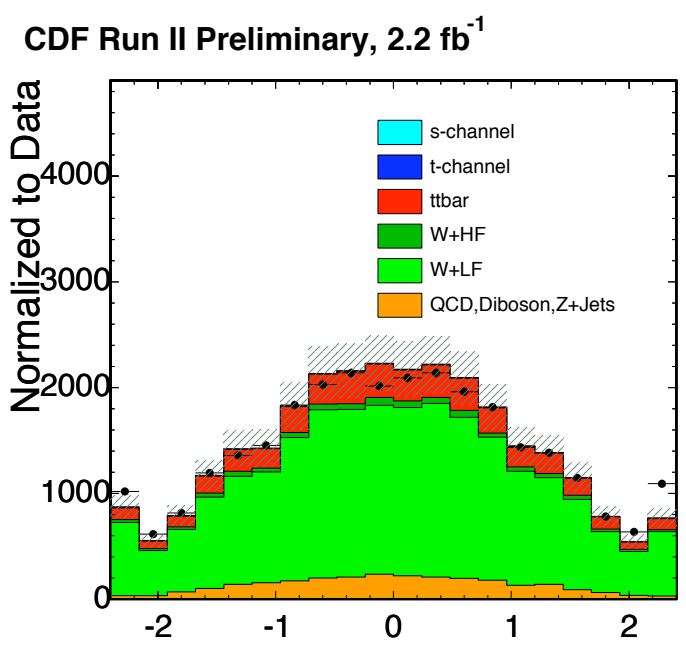

$\eta$ of the second highest momentum jet CDF Run II Preliminary, $2.2 \mathrm{fb}^{-1}$

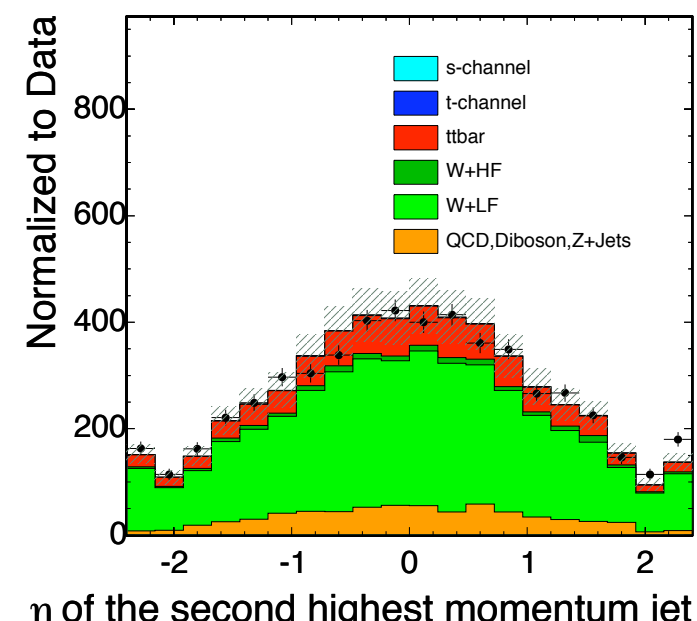

Figure 7.45: Stacked histogram of $\eta$ of the second jet. Data is compared to the prediction with each component normalized to its predicted value, shown in the 2-jet taggable region (above) and 3-jet taggable region (below). The mismodeling in the high $|\eta|$ region indicates the need for the modeling systematic taken for this distribution 


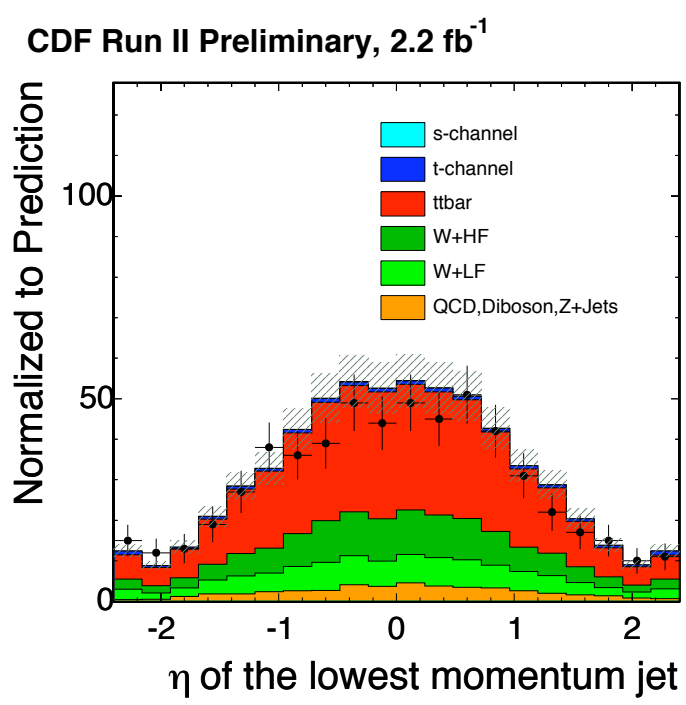

Figure 7.46: Stacked histogram of $\eta$ of the third jet. Data is compared to the prediction with each component normalized to its predicted value, shown in the 3 -jet tag region. For 3-jet plots, this is the same distribution as the $\eta$ of the lowest momentum jet, for which a modeling systematic is applied. 


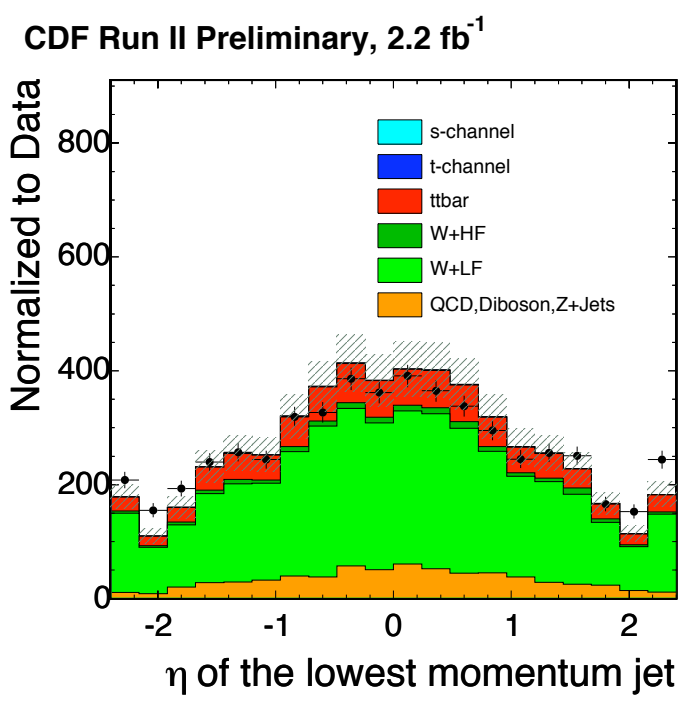

Figure 7.47: Stacked histogram of $\eta$ of the third jet. Data is compared to the prediction with each component normalized to its predicted value, shown in the 3-jet taggable region. The mis-modeling in the high $|\eta|$ region indicates the need for the modeling systematic taken for this distribution 

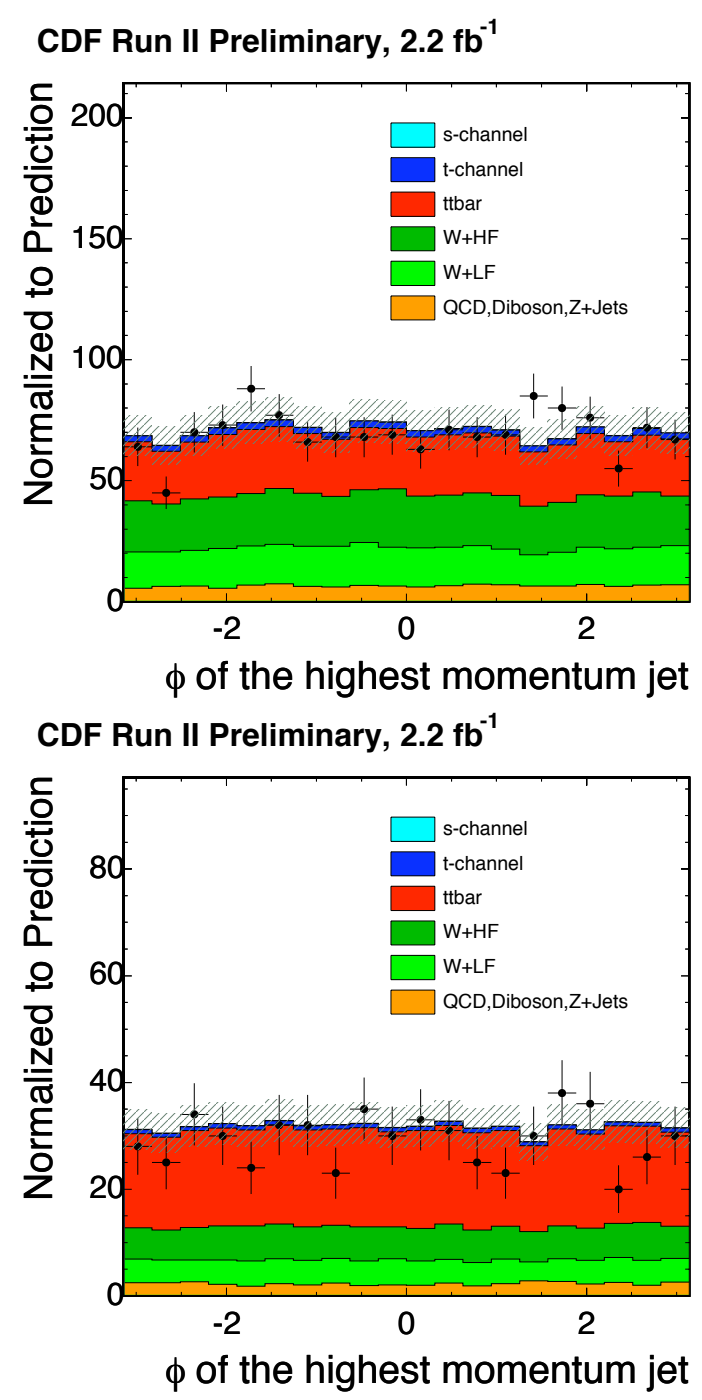

Figure 7.48: Stacked histogram of $\phi$ of the lowest momentum jet. Data is compared to the prediction with each component normalized to its predicted value, shown in the 2-jet tag region (above) and 3-jet tag region (below). The modeling for $\phi$ of the lowest momentum jet looks fine in both plots, and the KS test values shown in Table 7.1 are not suspiciously low for this variable. 


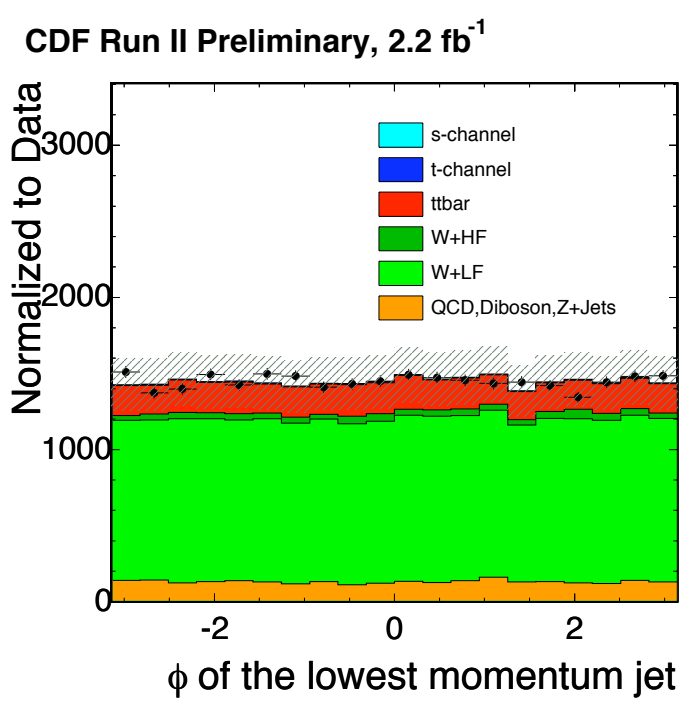

CDF Run II Preliminary, $2.2 \mathrm{fb}^{-1}$

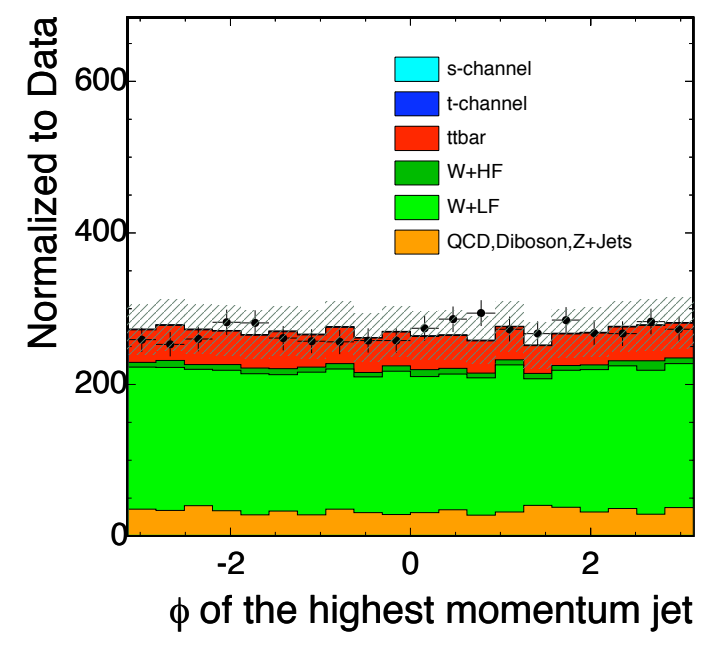

Figure 7.49: Stacked histogram of $\phi$ of the lowest momentum jet. Data is compared to the prediction with each component normalized to its predicted value, shown in the 2-jet taggable region (above) and 3-jet taggable region (below). The modeling for $\phi$ of the lowest momentum jet looks fine in both plots. 

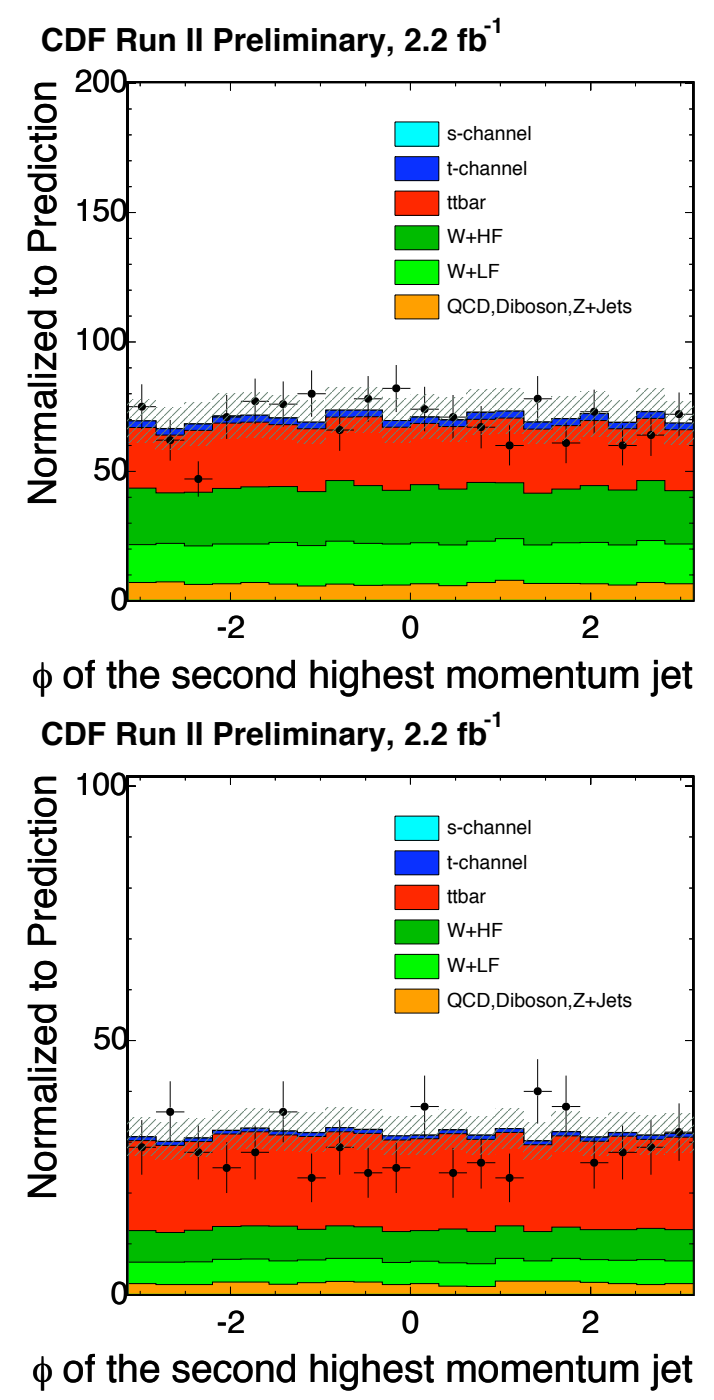

Figure 7.50: Stacked histogram of $\phi$ of the second jet. Data is compared to the prediction with each component normalized to its predicted value, shown in the 2 -jet tag region (above) and 3-jet tag region (below). The modeling for $\phi$ of the second jet looks fine in both plots, and the KS test values shown in Table 7.1 are not suspiciously low for this variable. 


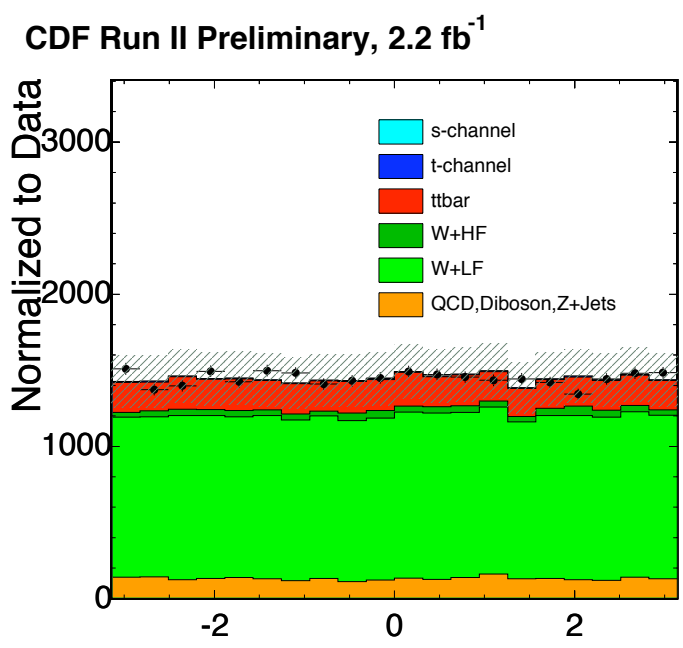

$\phi$ of the second highest momentum jet CDF Run II Preliminary, $2.2 \mathrm{fb}^{-1}$

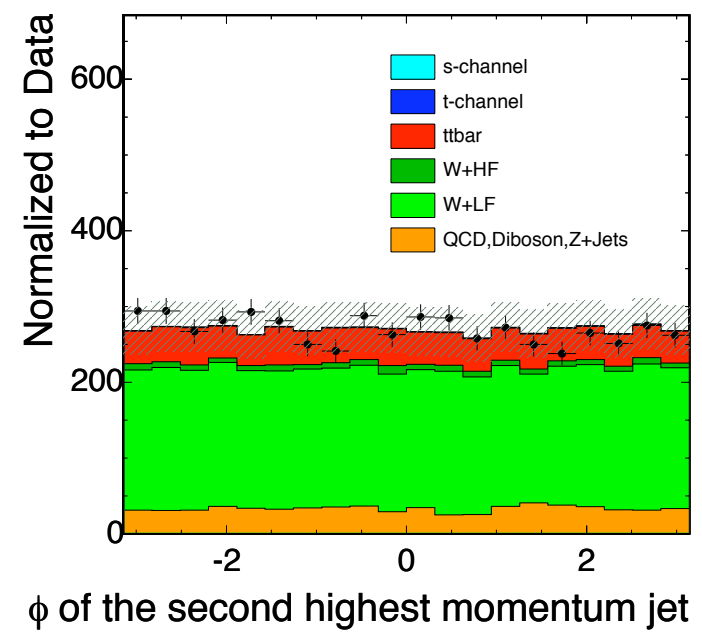

Figure 7.51: Stacked histogram of $\phi$ of the second jet. Data is compared to the prediction with each component normalized to its predicted value, shown in the 2-jet taggable region (above) and 3-jet taggable region (below). The modeling for $\phi$ of the second jet looks fine in both plots. 


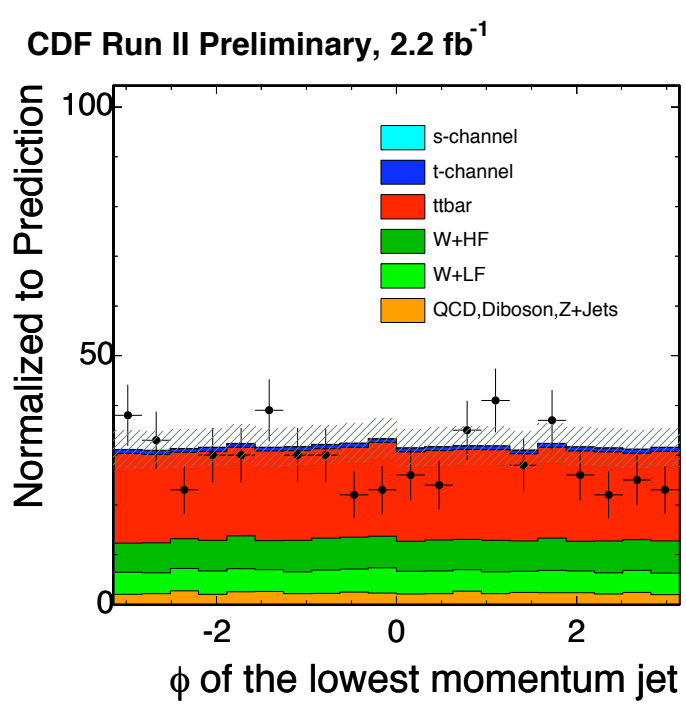

Figure 7.52: Stacked histogram of $\phi$ of the third jet. Data is compared to the prediction with each component normalized to its predicted value, shown in the 3 -jet tag region. The modeling for $\phi$ of the third jet looks fine in both plots, and the KS test values shown in Table 7.1 are not suspiciously low for this variable.

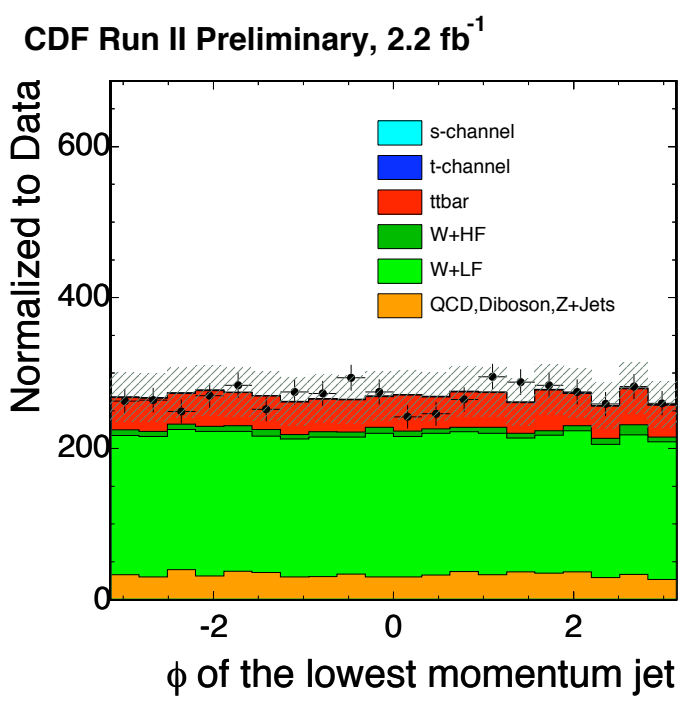

Figure 7.53: Stacked histogram of $\phi$ of the third jet. Data is compared to the prediction with each component normalized to its predicted value, shown in the 3-jet taggable region. The modeling for $\phi$ of the third jet looks fine in both plots. 

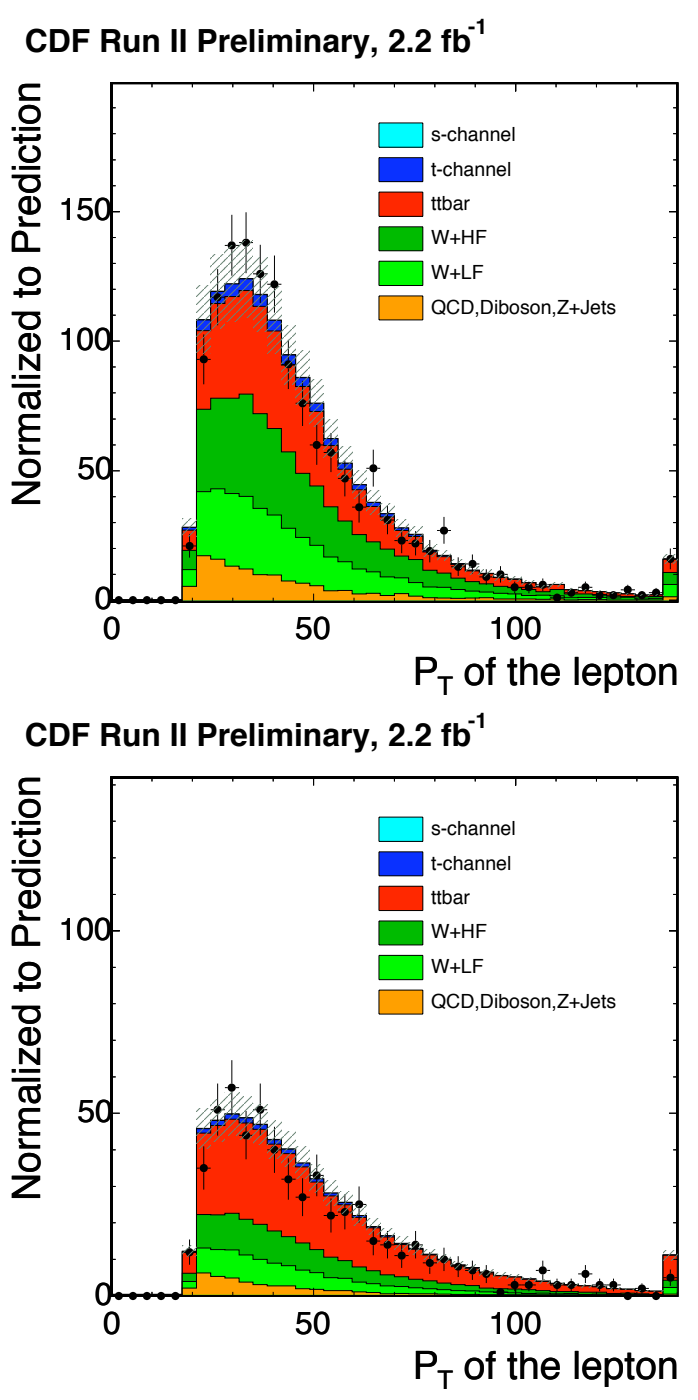

Figure 7.54: Stacked histogram of $P_{T}$ of the lepton. Data is compared to the prediction with each component normalized to its predicted value, shown in the 2-jet tag region (above) and 3-jet tag region (below). The modeling for $P_{T}$ of the lepton looks fine in both plots, and the KS test values shown in Table 7.1 are not suspiciously low for this variable. 

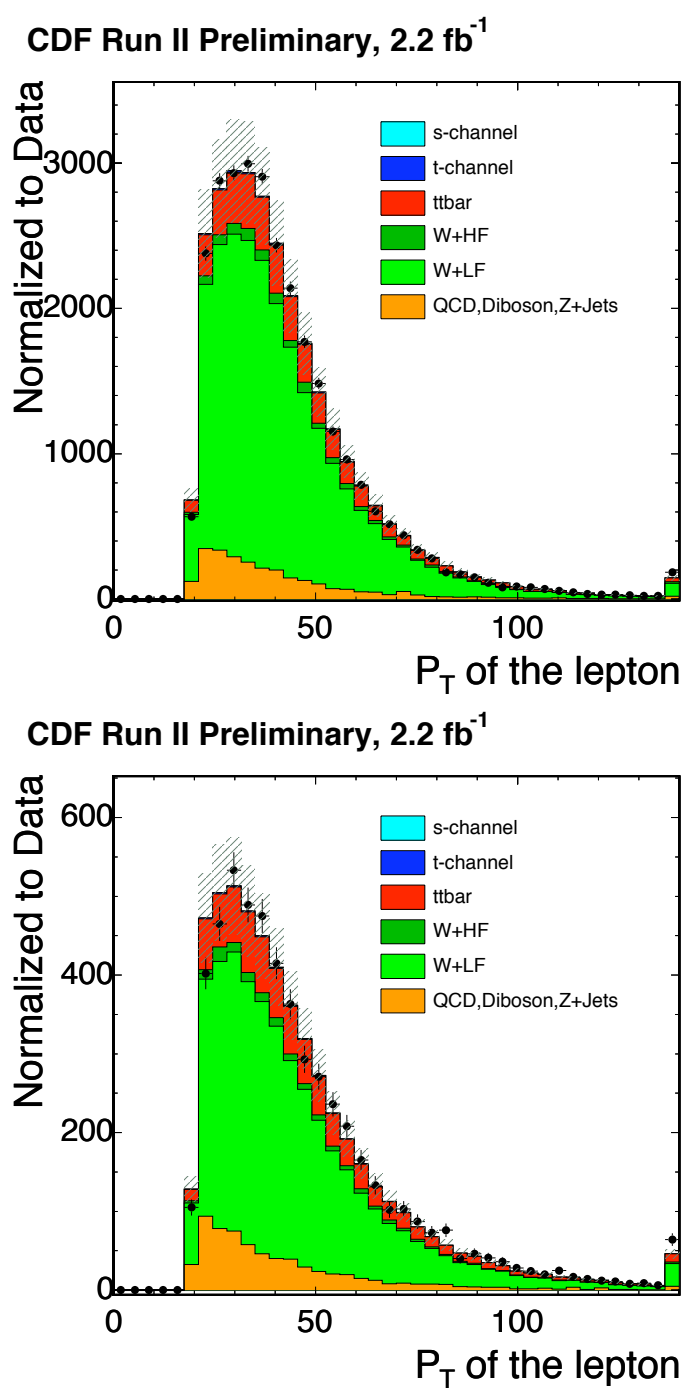

Figure 7.55: Stacked histogram of $P_{T}$ of the lepton. Data is compared to the prediction with each component normalized to its predicted value, shown in the 2-jet taggable region (above) and 3-jet taggable region (below). The modeling for $P_{T}$ of the lepton looks fine in both plots. 


\section{Systematics and Statistical
Methods}

\subsection{Systematics}

In the single top analysis, it is important to consider and carefully quantify sources of systematic uncertainty. For this case, systematics from a number of sources are considered. These sources include Monte Carlo and other modeling uncertainties, and uncertainties relating to particle properties like mass and cross section. Detector effects considered include the modeling of instrumental background. The luminosity uncertainty is a mix of detector effects and a theoretical uncertainty on the $p \bar{p}$ cross section. The uncertainty on the event selection efficiency includes a number of detector and modeling effects, as described in Section 5.3.2. Other Monte Carlo effects considered include uncertainty in the modeling of initial state radiation and final state radiation (ISR and FSR), uncertainties on the jet energy scale (JES), the parton distribution function used (PDF), the choice of Monte Carlo event generator, and the factorization and renormalization scale for the simulation of $\mathrm{W}+\mathrm{HF}$ events taken as a shape systematic on $\mathrm{W} b \bar{b}$. Two types of systematics are assessed relating to the neural network $b$-tagger, including variations in the neural network $b$ tag flavor content assumptions for QCD and mistag backgrounds, and a modeling systematic based on the calculated $b$-tagger correction factors. Explicit modeling systematics are taken for two variables $\left(\Delta R_{j j}\right.$ and $\eta$ jet 2$)$ that were found to be mismodeled in sideband data samples. Finally, the uncertainty on the $t \bar{t}$ cross section prediction and mass are also taken as systematics.

Both rate and shape systematics are used in this analysis. Depending on the systematic, either a rate only, rate and shape, or shape only systematic is evaluated. In general, When a source affects two analysis quantities the effects are treated as 100\% correlated. Systematic errors from different sources are treated as uncorrelated. Rate and shape uncertainties are taken to be correlated when they are from the same source. Systematic uncertainties on both the rates and shapes are allowed to be asymmetric. All shape templates used are shown in Appendix B. For the systematics, the priors used in the Bayesian technique for measuring the single-top cross section are given. This technique is further described in Section 8.2.1.

Several rate only systematics are evaluated. These include a $6 \%$ uncertainty on the integrated luminosity measurment, the $b$-tag scale factor and $K$ factor uncertainties (as described in Chapter 4), and the uncertainty on the $t \bar{t}$, single 
top and diboson cross section predictions and the uncertainty on Z+Jets production rate. Other rate only systematics include a single top model systematic taken by comparing the single top Monte Carlo with a full NLO Monte Carlo prediction, the uncertainty on the top mass, and the event selection efficiency uncertainty (Chapter 4).

Several additional systematics have both rate and shape variations. These include JES, PDF, ISR/FSR, the top mass systematic, and the factorization and renormalization scale systematic.

The jet energy correction systematic is created by varying the jet energy corrections within their $\pm 1 \sigma$ uncertainties. Both rate systematics and shape systematics were evaluated. For the shape systematic, alternative templates were calculated for all signal and background processes. This technique is similar to that discussed in [85]. These templates were taken as $\pm 1 \sigma$ variations applied with untruncated Gaussian priors.

The ISR and FSR systematics are estimated by producing samples in which the Monte Carlo setting was altered to produce either more or less initial-state radiation and final-state radiation. The settings used for this systematic were derived from studies of ISR using Drell-Yan events [85]. Shape and rate systematics were used for single top and $t \bar{t}$ events. The ISR and FSR systematics were taken to be correlated. For the shape uncertainty, the templates were used as $\pm 1 \sigma$ variations with untruncated Gaussian priors.

The PDF parameterization systematic was studied by re-weighted single top and $t \bar{t}$ events with weights associated with various PDF sets including 20 CTEQ6M eigenvectors with up and down $\pm 1 \sigma$ variations, and PDFs from the MRST and CTEQ groups: MRST72, MRST75, CTEQ6L, CTEQ6L1. Both a rate and shape systematic was evaluated for the PDF parametrization, and the templates for the shape systematics were taken to be $\pm 1 \sigma$ variations with untruncated Gaussian priors.

A shape systematic and rate for the factorization and renormalization scale of the $\mathrm{W} b \bar{b}$ sample was created by varying the renormalization scale of the Alpgen Monte Carlo simulation of $\mathrm{W} b \bar{b}$ [73]. The renormalization scale was doubled for the $+1 \sigma$ variation and halved for the $-1 \sigma$ variation. Both rate and shape systematics were evaluated. As with the previous shape systematics, the templates for the shape systematics were taken to be $\pm 1 \sigma$ variations truncated Gaussian priors truncated at $\pm 1 \sigma$.

The final systematic category is shape-only systematics. These include systematics taken for the shape of the neural network $b$-tagger distribution, the neural network flavor assignment to the QCD model, and a systematic taken for disagreement seen between the data and Monte Carlo model for certain taggable samples

Only shape systematics were taken for the shape of the neural network $b$ tagger. These systematics were taken from the 'optimistic' and 'pessimistic' scenarios discussed in Section 6.3. For the 'optimistic' systematic, the light 
flavor correction function is not applied to the mistag jets, while for the 'pessimistic', systematic the mistag correction function is applied to $W c \bar{c}$ and $W c$ templates. This is evaluated using a truncated Gaussian prior where the pessimistic variation is taken to be $-1 \sigma$ and the optimistic variation is taken to be $+1 \sigma$.

Similarly, a shape systematic is assessed for the neural net $b$-tagger flavor assigned to the QCD model. The weights used for the assignment of $b$-tag types are varied from the central values of $45 \% \mathrm{~b}, 40 \% \mathrm{c}$ and $15 \%$ light flavor to the alternate values of $60 \% \mathrm{~b}, 30 \% \mathrm{c}, 10 \%$ light flavor. A flat box prior was used for the evaluation of this shape systematic.

Another shape-only systematic was the modeling of the mistagged lightquark jets. For this systematic, an alternative model was used for the template histograms, simply created from the measured $\mathrm{W}+$ Jets events before $b$-tagging. The prior for this systematic was taken to be a truncated half-Gaussian with the alternative model given a $1 \sigma$ variation.

Shape uncertainties are also assessed for the mis-modeling of the $\Delta R_{j j}$ and $\eta_{j 2}$ distributions seen in the taggable samples. In the 3 -jet bin, the $\Delta R_{j j}$ systematic was found to be reasonably well modeled in the taggable region, and thus the systematic was not applied. For this systematic, an alternate model was used in which the distributions for all signal and background types were reweighted by the difference between the taggable distribution and the taggable model. For these shape systematics, a truncated half-Gaussian prior was used between the Monte Carlo and taggable data shapes.

The rate systematics evaluated for this analysis are given in Tables 8.1 and 8.2 , for the two and three jet bins respectively. Shape systematics are shown in Appendix B. A median smoothing algorithm with a window of five bins is applied to all shape systematics in order to reduce the sensitivity to limited Monte Carlo statistics. This smoothing algorithm is applied to the ratio between the shape varied and central samples as follows: for each bin, the median of the five bins centered on that bin is calculated, and the content of the bin is replace by this median value. For the first and last two bins, no smoothing is applied. After smoothing, the ratio template is re-multiplied by the central sample and then normalized to have the same integral as the central sample. 


\begin{tabular}{|c|c|c|c|c|c|}
\hline Source & $t$-channel & $s$-channel & $\bar{t} \bar{t}$ & $W+\mathrm{LF}$ & \\
\hline JES -/+ & $-1.1 / 0.6 \mathrm{~s}$ & $-0.1 /-0.6 \mathrm{~s}$ & $9.7 /-9.3 \mathrm{~s}$ & $\mathrm{~s}$ & \\
\hline ISR more/less & $-0.2 / 2.8 \mathrm{~s}$ & $6.7 / 0.3 \mathrm{~s}$ & $-7.1 /-2.6 \mathrm{~s}$ & - & \\
\hline FSR more/less & $-1.3 / 4.2 \mathrm{~s}$ & $0.4 / 5.9 \mathrm{~s}$ & $-2.6 /-5.1 \mathrm{~s}$ & - & \\
\hline PDF & $-3.5 / 3.1 \mathrm{~s}$ & $-1.4 / 1.7 \mathrm{~s}$ & $-2.3 / 1.9 \mathrm{~s}$ & - & \\
\hline Signal MC model & $-2.0 / 2.0$ & $-1.0 / 1.0$ & - & - & \\
\hline Luminosity & $-6.0 / 6.0$ & $-6.0 / 6.0$ & $-6.0 / 6.0$ & - & \\
\hline Efficiency & $-4.2 / 4.2$ & $-2.3 / 2.3$ & $-2.6 / 2.6$ & - & \\
\hline Cross Section (t) & $-12.6 / 12.6$ & $-12.4 / 12.4$ & - & - & \\
\hline Cross Section (tt) & - & - & $-12.4 / 12.4$ & - & \\
\hline Cross Section (dib) & - & - & - & - & \\
\hline$Z+$ jets rate & - & - & - & - & \\
\hline$m_{t}(170 / 180)$ & $6.1 /-5.3 \mathrm{~s}$ & $9.5 /-8.0 \mathrm{~s}$ & $7.8 /-8.1 \mathrm{~s}$ & - & \\
\hline$W+\mathrm{QQ} k$-factor & - & - & - & - & \\
\hline$W+\mathrm{c} k$-factor & - & - & - & - & \\
\hline$\eta_{j 2}$ & $\mathrm{~s}$ & $\mathrm{~s}$ & $\mathrm{~s}$ & $\mathrm{~s}$ & \\
\hline$\Delta R_{j j}$ & $\mathrm{~s}$ & $\mathrm{~s}$ & $\mathrm{~s}$ & $\mathrm{~s}$ & \\
\hline DataMistags & - & - & - & $\mathrm{s}$ & \\
\hline KIT Flav. Sep. & - & - & - & $\mathrm{s}$ & \\
\hline Non-W Rate & - & - & - & - & \\
\hline \multirow[t]{2}{*}{ Non-W Flavor } & - & - & - & - & \\
\hline & $\overline{W b b}$ & $\overline{W c(c)}$ & Diboson & $Z+$ jets & $\overline{\text { Non- } W}$ \\
\hline JES -/+ & $6.9 /-7.6 \mathrm{~s}$ & $7.0 /-6.3 \mathrm{~s}$ & $-2.6 / 1.7 \mathrm{~s}$ & $-5.3 / 5.4 \mathrm{~s}$ & - \\
\hline ISR more/less & - & - & - & - & - \\
\hline FSR more/less & - & - & - & - & - \\
\hline $\mathrm{PDF}$ & - & - & - & - & - \\
\hline Signal MC model & - & - & - & - & - \\
\hline Luminosity & - & - & $-6.0 / 6.0$ & $-6.0 / 6.0$ & - \\
\hline Efficiency & - & - & $-9.0 / 9.0$ & $-8.3 / 8.3$ & - \\
\hline Cross Section (t) & - & - & - & - & - \\
\hline Cross Section (tt) & - & - & - & - & - \\
\hline Cross Section (dib) & - & - & $-2.0 / 2.0$ & - & - \\
\hline$Z+$ jets rate & - & - & - & $-10.8 / 10.8$ & - \\
\hline$m_{t}(170 / 180)$ & - & - & - & - & - \\
\hline$W+\mathrm{QQ} k$-factor & $-30.1 / 30.1$ & $-13.9 / 13.9$ & - & - & - \\
\hline$W+\mathrm{c} k$-factor & - & $-16.1 / 16.1$ & - & - & - \\
\hline$\eta_{j 2}$ & s & $\mathrm{s}$ & $\mathrm{s}$ & $\mathrm{s}$ & - \\
\hline$\Delta R_{j j}$ & $\mathrm{~S}$ & $\mathrm{~s}$ & $\mathrm{~s}$ & $\mathrm{~s}$ & - \\
\hline DataMistags & - & - & - & - & - \\
\hline KIT Flav. Sep. & - & $\mathrm{s}$ & - & - & - \\
\hline Non-W Rate & - & - & - & - & $-40.0 / 40.0$ \\
\hline Non-W Flavor & - & - & - & - & $\mathrm{S}$ \\
\hline
\end{tabular}

Table 8.1: Systematic rate uncertainties for the 2-jet bin, in percent. Shape uncertainties are indicated with an "s". 


\begin{tabular}{|c|c|c|c|c|c|}
\hline Source & $t$-channel & $s$-channel & $t \bar{t}$ & $W+\mathrm{LF}$ & \\
\hline JES - / + & $-9.7 / 2.5 \mathrm{~s}$ & $-8.0 / 8.9 \mathrm{~s}$ & $4.6 /-5.1 \mathrm{~s}$ & $\mathrm{~S}$ & \\
\hline ISR more/less & $-0.5 /-4.5 \mathrm{~s}$ & $-12.6 / 2.9 \mathrm{~s}$ & $-5.0 /-0.6 \mathrm{~s}$ & - & \\
\hline FSR more/less & $-2.5 /-0.8 \mathrm{~s}$ & $-4.8 /-6.3 \mathrm{~s}$ & $-2.3 /-3.5 \mathrm{~s}$ & - & \\
\hline PDF & $-3.7 / 3.3 \mathrm{~s}$ & $-1.5 / 1.7 \mathrm{~s}$ & $-2.3 / 1.9 \mathrm{~s}$ & - & \\
\hline Signal MC model & $-1.5 / 1.5$ & $-1.9 / 1.9$ & - & - & \\
\hline Luminosity & $-6.0 / 6.0$ & $-6.0 / 6.0$ & $-6.0 / 6.0$ & - & \\
\hline Efficiency & $-4.3 / 4.3$ & $-3.9 / 3.9$ & $-4.0 / 4.0$ & - & \\
\hline Cross Section (t) & $-12.6 / 12.6$ & $-12.4 / 12.4$ & - & - & \\
\hline Cross Section (tt) & - & - & $-12.4 / 12.4$ & - & \\
\hline Cross Section (dib) & - & - & - & - & \\
\hline$Z+$ jets rate & - & - & - & - & \\
\hline$m_{t}(170 / 180)$ & $6.4 /-6.0 \mathrm{~s}$ & $11.7 /-8.6 \mathrm{~s}$ & $9.3 /-8.6 \mathrm{~s}$ & - & \\
\hline$W+\mathrm{QQ} k$-factor & - & - & - & - & \\
\hline$W+\mathrm{c} k$-factor & - & - & - & - & \\
\hline$\eta_{j 3}$ & $\mathrm{~s}$ & $\mathrm{~s}$ & $\mathrm{~s}$ & $\mathrm{~s}$ & \\
\hline Data Mistags & - & - & - & $\mathrm{s}$ & \\
\hline KIT Flav. Sep. & - & - & - & $\mathrm{s}$ & \\
\hline Non-W Rate & - & - & - & - & \\
\hline \multirow[t]{2}{*}{ Non-W Flavor } & - & - & - & - & \\
\hline & $\overline{\overline{W b b}}$ & $\overline{W c(c)}$ & Diboson & $\overline{Z+\text { jets }}$ & $\overline{\text { Non- } W}$ \\
\hline JES $-/+$ & $8.8 /-8.8 \mathrm{~s}$ & $8.3 /-7.1 \mathrm{~s}$ & $-13.0 / 10.8 \mathrm{~s}$ & $-10.5 / 13.6 \mathrm{~s}$ & - \\
\hline ISR more/less & - & - & - & - & - \\
\hline FSR more/less & - & - & - & - & - \\
\hline PDF & - & - & - & - & - \\
\hline Signal MC model & - & - & - & - & - \\
\hline Luminosity & - & - & $-6.0 / 6.0$ & $-6.0 / 6.0$ & - \\
\hline Efficiency & - & - & $-9.0 / 9.0$ & $-8.0 / 8.0$ & - \\
\hline Cross Section (t) & - & - & - & - & - \\
\hline Cross Section (tt) & - & - & - & - & - \\
\hline Cross Section (dib) & - & - & $-2.0 / 2.0$ & - & - \\
\hline$Z+$ jets rate & - & - & - & $-10.8 / 10.8$ & - \\
\hline$m_{t}(170 / 180)$ & - & - & - & - & - \\
\hline$W+\mathrm{QQ} k$-factor & $-30.1 / 30.1$ & $-17.7 / 17.7$ & - & - & - \\
\hline$W+\mathrm{c} k$-factor & - & $-12.3 / 12.3$ & - & - & - \\
\hline$\eta_{j 3}$ & $\mathrm{~s}$ & $\mathrm{~S}$ & $\mathrm{~s}$ & $\mathrm{~s}$ & - \\
\hline Data Mistags & - & - & - & - & - \\
\hline KIT Flav. Sep. & - & $\mathrm{s}$ & - & - & - \\
\hline Non-W Rate & - & - & - & - & $-40.0 / 40.0$ \\
\hline Non-W Flavor & - & - & - & - & $\mathrm{s}$ \\
\hline
\end{tabular}

Table 8.2: Systematic rate uncertainties for the 3-jet bin, in percent. Shape uncertainties are indicated with an "s". 


\subsection{Statistical Methods}

The goal of the multivariate likelihood search for single top quark production is to ultimately discover single top production and measure the best central value of the signal cross sections. Each of these goals requires the use of different statistical techniques. To measure the significance of the measurement $p$-value is calculated using the technique of marginalizing the probability distribution functions of the test statistic, or using the "prior-predictive ensemble", similar to the technique described in References [86] and [87]. For the measurement of the cross section best fit, the likelihood function is minimized over allowed values of the nuisance parameters using a Bayesian technique [21].

\subsubsection{Likelihood Function}

The likelihood function gives the probability of observing the data events obtained. It is a function of the Poisson means for signal and background, and the values of these means at which the likelihood achieves its maximum correspond to the most probable estimate for the true signal and background content of the data.

For this analysis, a binned likelihood fit to the multivariate likelihood is performed. A discussion of similar techniques can be found in Reference [21]. This likelihood function, $\mathcal{L}$ is given by:

$$
\mathcal{L}=\prod_{k=1}^{n b i n s} \frac{e^{-\mu_{k}} \mu_{k}^{n_{k}}}{n_{k} !} \cdot \prod_{j}^{b k g} G_{j}\left(\beta_{j} \mid \Delta_{j}\right) \cdot \prod_{i}^{s y s} G_{i}\left(\delta_{i}\right)
$$

Here, the expected number of events in bin $k, \mu_{k}$, is given by:

$$
\begin{gathered}
\mu_{k}=\sum_{j}^{\text {process }} \beta_{k} \cdot \sum_{i}^{s y s}\left[1+\left|\delta_{i}\right| \cdot\left(\epsilon_{j i+} H\left(\delta_{i}\right)+\epsilon_{j i-} H\left(-\delta_{i}\right)\right)\right] \\
\cdot \alpha_{j k} \cdot \sum_{i}^{s y s}\left[1+\left|\delta_{i}\right| \cdot\left(\kappa_{j i k+} H\left(\delta_{i}\right)+\kappa_{j i k-} H\left(-\delta_{i}\right)\right)\right]
\end{gathered}
$$

The parameter $\beta_{j}=\sigma_{j}^{f i t} / \sigma_{j}^{\mathrm{SM}}$. Here, the sum over the processes is taken over all the background and signal components for the cross-section measurement, and over either the background or background and signal components for the p-value calculation, depending on the test hypothesis. $\Delta_{j}$ are the Gaussian constraints for the background components, taken from the background predictions given in Section 5.5.2. The shape information is given by, $\alpha_{j k}$, which is the content of the template bin $\mathrm{j}$ of process $\mathrm{k}$. The relative strength of a systematic effect for source $i$ is given by $\delta_{i}$. The $\pm 1 \sigma$ changes in the normalization of process $j$ given by the $i^{t h}$ source of systematic uncertainty are given by $\epsilon_{j i \pm}$, and the $\pm 1 \sigma$ shape uncertainties are given by $\kappa_{j i \pm} . H\left(\delta_{i}\right)$ is the Heaviside function.

The Gaussian constraints on the backgrounds $G_{j}$ are summed over all backgrounds and the Gaussian constraints on the systematics $G_{i}$ are summed over 
all systematics used. Here, for instance:

$$
G_{j}\left(\beta_{j} \mid \Delta_{j}\right)=\frac{1}{\sqrt{2 \pi \cdot \Delta_{j}^{2}}} \exp \left(-\frac{1}{2} \cdot\left(\frac{\beta_{j}-1.0}{\Delta_{j}}\right)^{2}\right)
$$

We marginalize the likelihood function by integrating $\mathcal{L}$ over the nuisance parameters, $\beta_{i}$ and $\delta_{i}$, for possible values of the single top cross section.

$$
\mathcal{L}_{R}\left(\beta_{1}\right)=\int \mathcal{L}_{R}\left(\beta_{i}, \delta_{i}\right) \pi\left(\beta_{i}, \delta_{i}\right) d \beta_{i} d \delta_{i}
$$

In this integration, the priors described in Section 8.1 are used for the systematics, and Gaussian priors are used for $\beta_{i}$. The resulting reduced likelihood, $\mathcal{L}_{R}\left(\beta_{1}\right)$ is a function of the single-top cross-section $\left(\beta_{1}\right)$ only.

\subsubsection{The $p$-value Calculation}

The degree to which we have evidence for single-top production is denoted by a $p$-value. The $p$ value is defined as the probability of observing data at least as signal-like as observed in the result in the absence of single top. In the calculation of this $p$-value, the 2 -jet bin and 3 -jet bin likelihood functions are combined to improve sensitivity. These 40 bin distributions shown in Section 7, Figures 7.6 and 7.12 .

A modified frequentist approach is used to determine the $p$-value in this analysis. For this approach, the data are compared with two hypotheses. The null hypothesis, or $H_{0}$ assumes the model is all Standard Model backgrounds as described in Section 5.5.2 except single-top production, while the test hypothesis $\left(H_{1}\right)$ assumes the model to be all Standard Model backgrounds and Standard Model single-top production, as described in Section 5.5.2.

The likelihood ratio is given by:

$$
-2 \ln Q=-2 \ln \frac{\mathcal{L}\left(H_{1}\right)}{\mathcal{L}\left(H_{0}\right)}
$$

Here, $\mathcal{L}$ is the likelihood described above, and $H_{1}$ is the signal and background hypothesis while $H_{0}$ is the background-only hypothesis. Two types of pseudexperiments are performed, one for the signal-plus background and the other for the background-only hypothesis. Pseudodata is drawn from Poisson distributions with the means set as the sum of the backgrounds, or the signal-plus backgrounds, depending on the hypothesis used. Predicted rates are not allowed to fluctuate negatively and a Bayesian interpretation of nuisance parameter priors called "prior-predictive ensemble" is used. The priors for each systematic are discussed in Section 8.1. The results of the $p$-value measurement are given in Section 9. 


\subsubsection{The Cross Section Measurement}

The second measurement made is a measurement of the top cross section. This is done using the maximum likelihood method. The posterior probability density function is given by:

$$
p\left(\beta_{1} \mid \text { data }\right)=\frac{\mathcal{L}_{R}\left(\text { data } \mid \beta_{1}\right) \pi\left(\beta_{1}\right)}{\int L_{R}\left(\text { data } \mid \beta_{1}^{\prime}\right) d \beta_{1}^{\prime}}
$$

Here, $L_{R}\left(\right.$ data $\left.\mid \beta_{1}\right)$ is the reduced likelihood and $\pi\left(\beta_{1}\right)$ is a flat prior in $\sigma_{s}+\sigma_{t}$. The Standard Model ratio between $\sigma_{s}$ and $\sigma_{t}$ is assumed. This reduced likelihood incorporates the same set of rate and shape systematics as described above, except the mass systematic and cross section uncertainties are neglected. In the creation of the reduced likelihood, all nuisance parameters are integrated over using the marginalization procedure described in Section 8.2.1. The measured cross section is given by the most probable value of $p\left(\beta_{1} \mid\right.$ data $)$ and the uncertainty is calculated using the range of highest posterior probability density which covers $68.27 \%$. The results of the cross section measurement are given in Section 9 .

A bias check is performed to check for the linearity of the cross section fit. In this case, random pseudoexperiments are generated by randomly fluctuating all systematics and extracting the cross section from each psudoexperiment. The results of this check show no bias to the analysis technique, and are shown in Figure 8.1. 


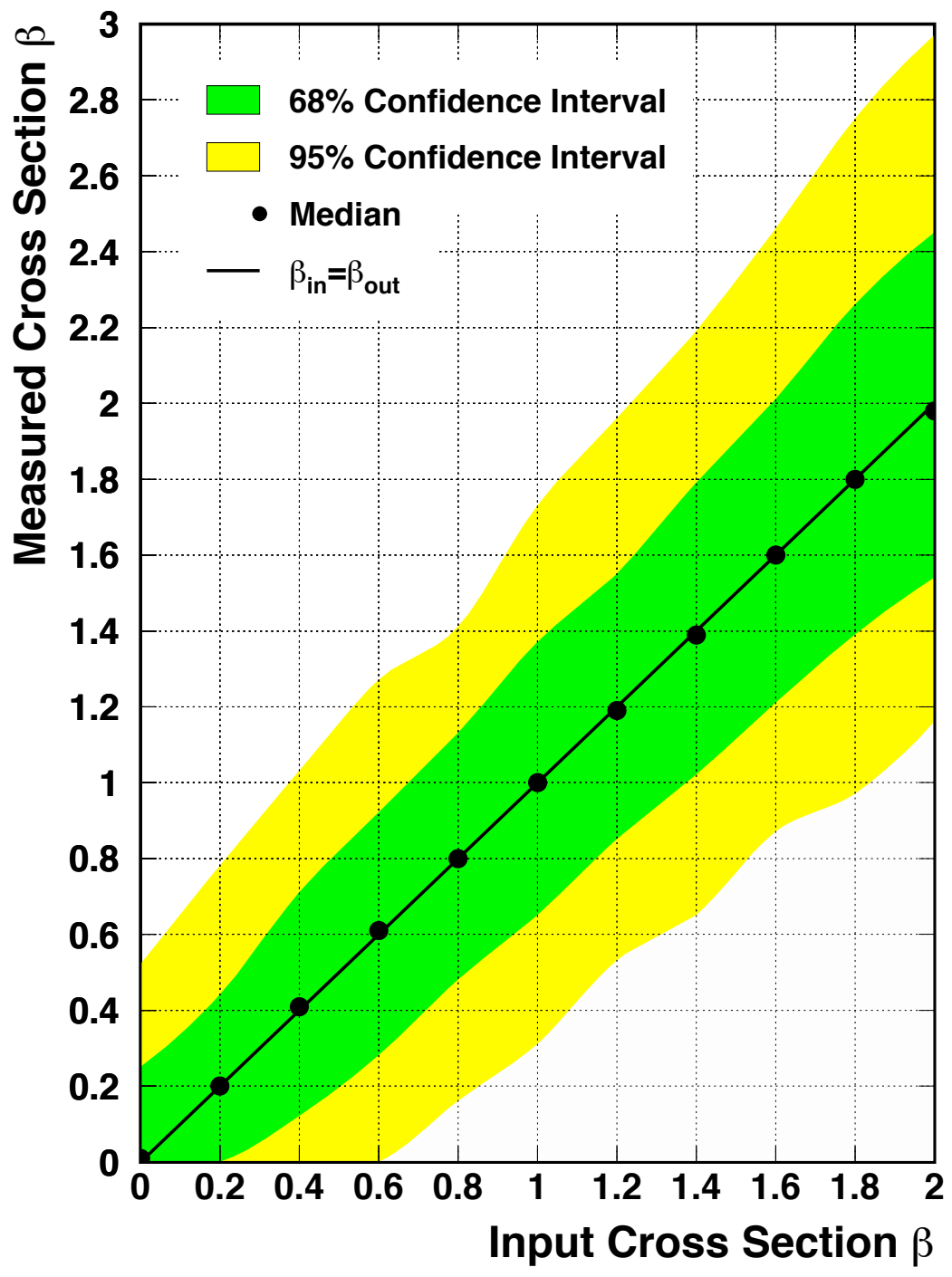

Figure 8.1: Linearity cross check. 


\section{Results}

\subsection{Expected and Observed $p$-value}

A modified frequentist approach is used in the calculation of the $p$-value for single-top production, as described in Section 8.2. The likelihood ratio, -2lnQ (given by $-2 \ln Q=-2 \ln \left(\frac{\mathcal{L}_{s i g+b k g}}{\mathcal{L}_{b k g}}\right)$ ) has the value $-2 \ln Q_{\text {obs }}=-4.79$ for the observed data, indicating a preference for the single-top hypothesis over the background-only hypothesis. The $p$-value is the probability that $-2 \ln Q<$ $-2 \ln Q_{\mathrm{obs}}$, assuming the background-only hypothesis

The $p$-value corresponding to the observed data is $2.40 \times 10^{-2}$, which indicates a $2.0 \sigma$ excess above the Standard Model background in the single-top region. This was calculated using fifty million pseudoexperiments.

The sensitivity of the analysis is computed as the median expected $p$-value assuming a signal is truly present at the Standard Model expected level. The median $-2 \ln Q$ is extracted from the signal-plus background distribution, and the integral of the background-only distribution of $-2 \ln Q$ to the left of this median value is the median expected $p$-value. The value thus obtained is $2.32 \times$ $10^{-4}$, corresponding to $3.5 \sigma$.

The observed and expected $p$-values are shown in Figure 9.1. 


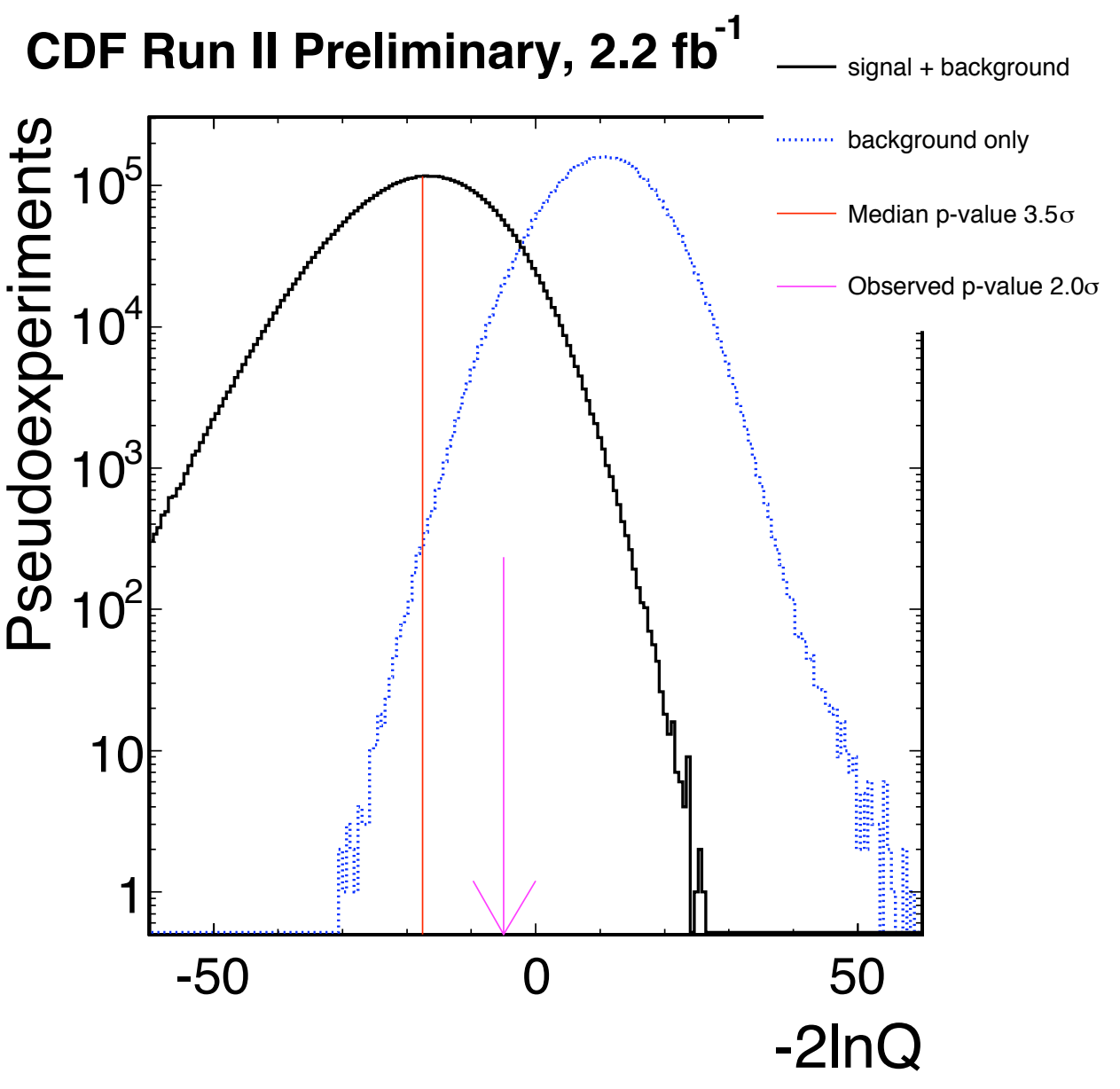

Figure 9.1: Plot of $-2 \ln Q$ for the test hypothesis, in which pseudoexperiments are drawn from a model which assumes Standard Model backgrounds plus Standard Model single-top production (solid histogram), and for the null hypothesis, in which pseudoexperiments are drawn from a model which assumes single-top production is absent (dashed histogram). The line indicates the median expected $p$-value of 0.000232 or $3.5 \sigma$ and the arrow indicates the data, with a $p$-value of 0.0240 or $2.0 \sigma$. 


\subsection{Measurement of the Single-Top Production Cross Section}

It is assumed that the modest observed excess of events over the expected background contribution is due to single top production, and a calculation is made of the production cross section. The measurement of the single-top production cross section is done using the Bayesian technique described in Section 8.2. Here, the posterior probability density was obtained using the marginalization procedure described in Section 8.2.3. A uniform prior in $\sigma_{s}+\sigma_{t}$ is assumed, and the SM ratio of $\sigma_{s} / \sigma_{t}$ is also assumed. This posterior probability density is shown in Figure 9.2. The maximum of the posterior is taken to be the bestfit value, and the $68 \%$ confidence interval is taken to be the shortest interval containing $68 \%$ of the integral of the posterior distribution. The resulting fit is $\sigma_{s}+\sigma_{t}=1.7_{-0.8}^{+0.9} \mathrm{pb}$.

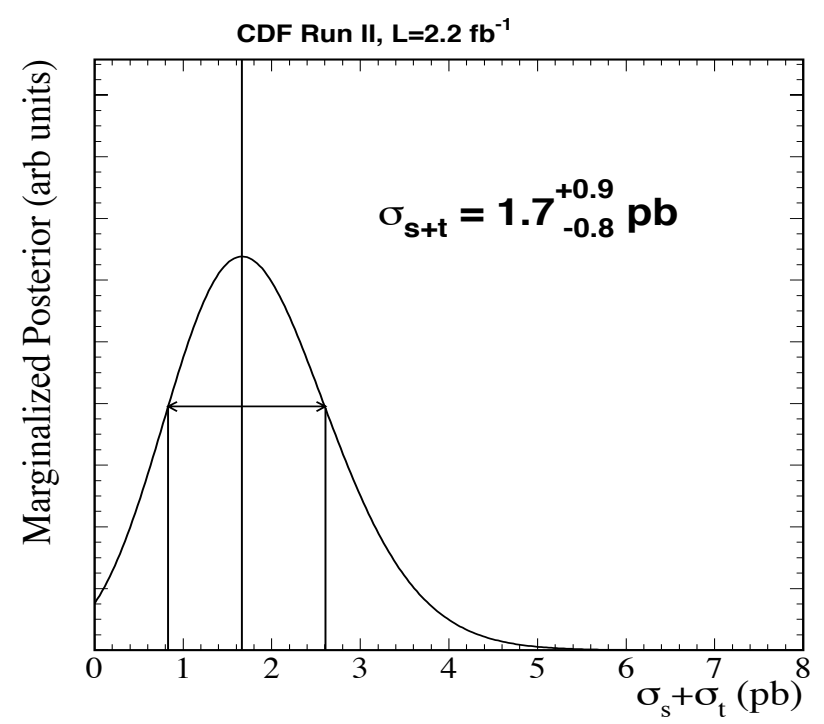

Figure 9.2: Fit for $\sigma_{s}+\sigma_{t}$. A uniform prior in $\sigma_{s}+\sigma_{t}$ is assumed, and the $\mathrm{SM}$ ratio of $\sigma_{s} / \sigma_{t}$ is also assumed. The Bayesian posterior, marginalized over nuisance parameters, is shown. The maximum value is the central value of the cross-section fit, and the smallest interval enclosing $68 \%$ of the integral of the posterior is the quoted interval. The measured result is $\sigma_{s}+\sigma_{t}=1.7_{-0.8}^{+0.9} \mathrm{pb}$.

\subsubsection{Measurement of $\left|V_{t b}\right|$}

A measurement of $V_{t b}$ can be extracted from the observed single top cross section. The relationship between the cross section and $V_{t b}$ is given by:

$\sigma_{s+t}^{\text {meas }} \simeq\left|V_{t b}^{\text {meas }}\right|^{2} \sigma_{s+t}^{\mathrm{SM}}$

For this calculation, the observed single top cross section is used, with its 


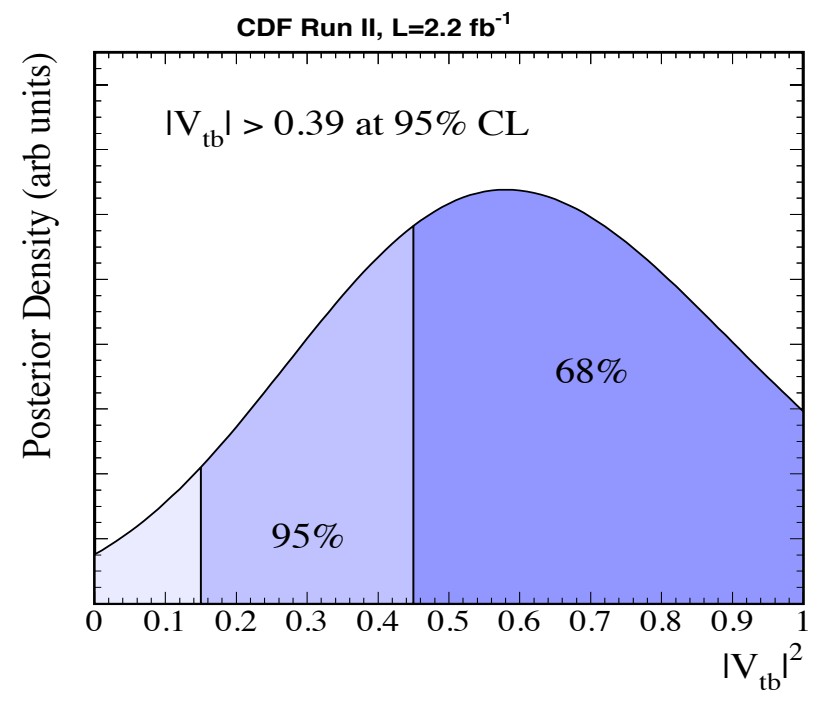

Figure 9.3: Extraction of limits on $\left|V_{t b}\right|^{2}$ using a Bayesian technique. A uniform prior in $\left|V_{t b}\right|^{2}$ is assumed, and the SM ratio of $\sigma_{s} / \sigma_{t}$ is also assumed. The Bayesian posterior, marginalized over nuisance parameters, including the theoretical uncertainty on the single-top cross section and uncertainties due to $m_{t}$, is shown. The $68 \%$ and $95 \%$ confidence intervals are indicated with vertical lines.

associated errors. This experimental errors on this measurement come from the measured uncertainty on observed cross section. An additional error of \pm 0.07 is added to account for the theoretical uncertainty on the single top cross section. This gives a total measurement of $\left|V_{t b}\right|=0.77_{-0.21}^{+0.18}$ (exp.) \pm 0.07 (theor.). In order to obtain a limit on $V_{t b}$, the cross-section prediction posterior density from Section 8.2.3 was recomputed including the single top cross-section prediction uncertainty. This was then used to compute a limit on $V_{t b}$ of $\left|V_{t b}\right|>0.39$ at the $95 \%$ confidence level, assuming a flat prior in $0<\left|V_{t b}\right|^{2}<1$. These results are shown in Figure 9.3. 


\section{0 outlook}

\subsection{CDF Results}

In addition to the multivariate likelihood search presented in this thesis, several multivariate searches for single-top have been completed. For the $2.2 \mathrm{fb}^{-1}$ data sample, four such searches have been completed: a multivariate likelihood search (this analysis), a neural network search, a matrix element search, and a boosted decision tree search [88] [89] [90]. In all four analyses, the cross section fit was low compared to the Standard Model expectation, ranging from a fit of $1.7_{-0.8}^{+0.9}$ for the likelihood function, to $2.3_{-0.7}^{+0.8}$ for the matrix element search.

An additional search for $s$-channel only was performed using $1.9 \mathrm{fb}^{-1}$ of data. This search fit a cross section for $s$-channel production of $0.6_{-0.6}^{+0.9}$, with an upper limit on anonymous $s$-channel production of $2.77 \mathrm{pb}$ at $95 \%$ C.L [91].

\subsection{CDF Combination}

A combination was made of three of the CDF results: the matrix element result, multivariate likelihood function result, and the neural network result. The outputs of the three discriminants were combined in an evolved neural network analysis. The combination takes advantage of the differences between each input analysis, and the final result has a higher significance than any individual result. However, like the individual analyses, the combination had a lower than expected significance. The CDF combination had an expected significance of $4.9 \sigma$ and an observed significance of $3.7 \sigma$. The cross section measured using this analysis was $2.2 \pm 0.7 \mathrm{pb}$. A plot of this result is shown in Figure 10.1 [92]. The cross section fit results of recent CDF searches are summarized in Figure 10.3

A measurement of $\left|V_{t b}\right|$ was also derived from the combination analysis, similar to the measurement described in Section 9.2.1. In this case, $\left|V_{t b}\right|$ was measured to be $0.88 \pm 0.14$ (exp) \pm 0.07 (theory). An upper limit, $\left|V_{t b}\right|>0,66$ at 95\% C.L was also calculated. These results are pictured in Figure 10.2.

\subsection{DØ Results}

Several searches for single-top have also been completed at $\mathrm{D} \varnothing$. The recent results include a decision tree, a matrix element, and a Bayesian neural network search, completed with $900 \mathrm{pb}^{-1}$ of data. A combination of the three results was 


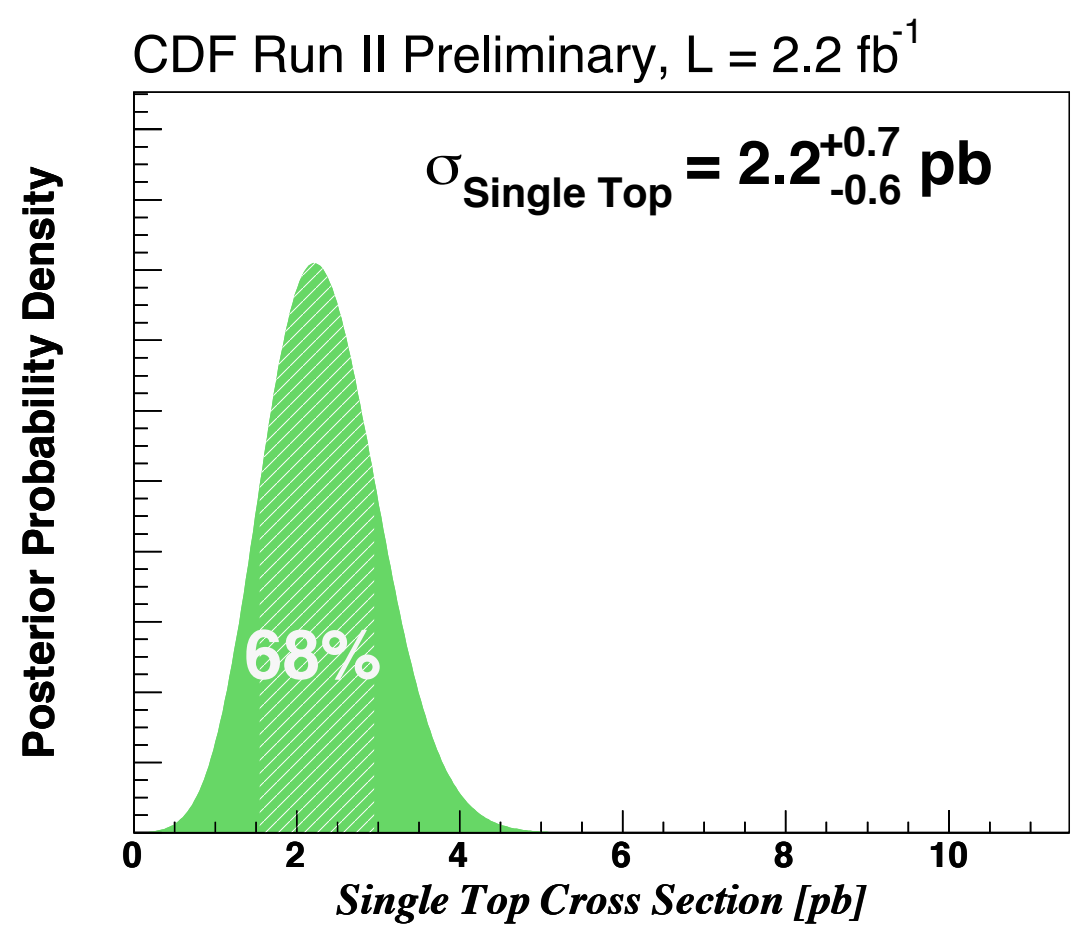

Figure 10.1: CDF combination cross section results [92].

made, resulting in a cross section of $4.7 \pm 1.3$ [2], [3] The cross section fit results of recent D $\varnothing$ and CDF searches are summarized in Figure 10.4.

The recent CDF searches fit slightly lower cross sections than the Standard Model expected, and the recent DØ searches fit slightly higher. However, in both cases the differences from the Standard Model are not statistically significant.

\subsection{Outlook at CDF}

If the Stadard Model predictions are correct, single-top quark production will likely be observed at the $5 \sigma$ confidence level at the Tevatron in the near future. Indeed, as mentioned above, the latest CDF combination result has a $4.9 \sigma$ expected significance, although the observed significance in data is somewhat lower. Figure 10.5 shows the expected significance of the CDF combination (labeled Winter 2008 combination), compared to a single analysis result (labeled Summer 2007 Single Channel). This shows that the combination can expect a significance of around $5 \sigma$ with $2 f b^{-1}$. However, extrapolating from the current result, a $5 \sigma$ significance is instead expected between $2-4 \mathrm{fb}^{-1}$, as shown in Figure 10.6. The bands in both plots shown $\pm 1 \sigma$ and $\pm 2 \sigma$ ranges on both predictions.

In conclusion, the search for single-top quarks at the CDF and at the Tevatron is entering an exciting era, with discovery literally around the corner. The 


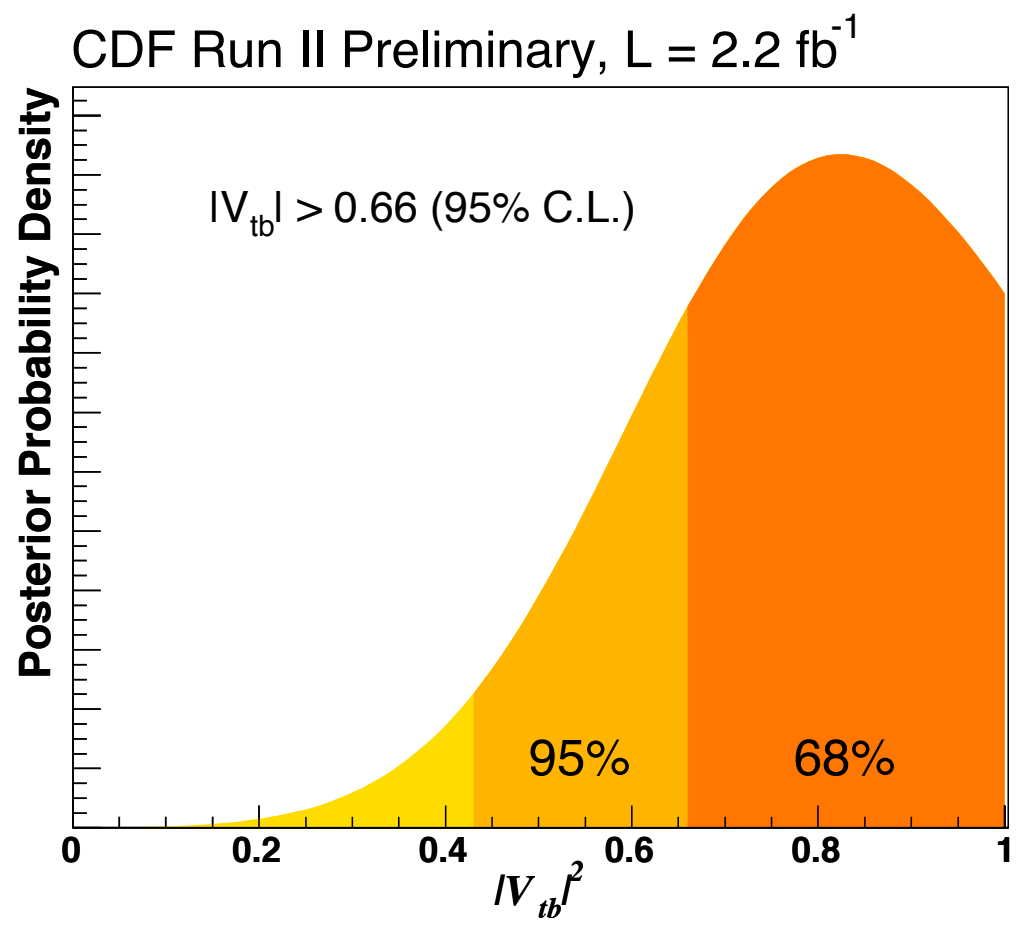

Figure 10.2: Measured value of $V_{t b}$ and upper limit using CDF Combination [92].

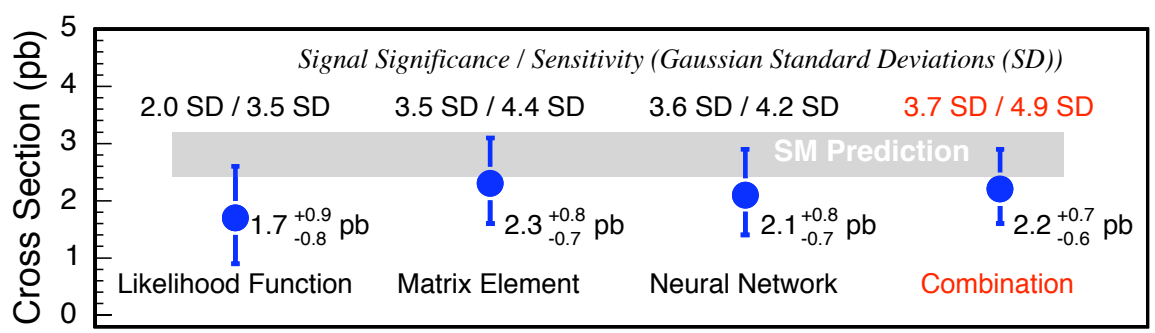

Figure 10.3: Measured Cross Sections from recent CDF measurements.

likelihood function and other multivariate searches employ a barrage of techniques allowing observation of single-top production with a much higher sensitivity than using a cut-based analysis alone. These searches are truly a technical tour de force, and provide a methodology for searching for small signals hidden behind large backgrounds with large applicability, both at the Tevatron and continuing into the LHC era. 


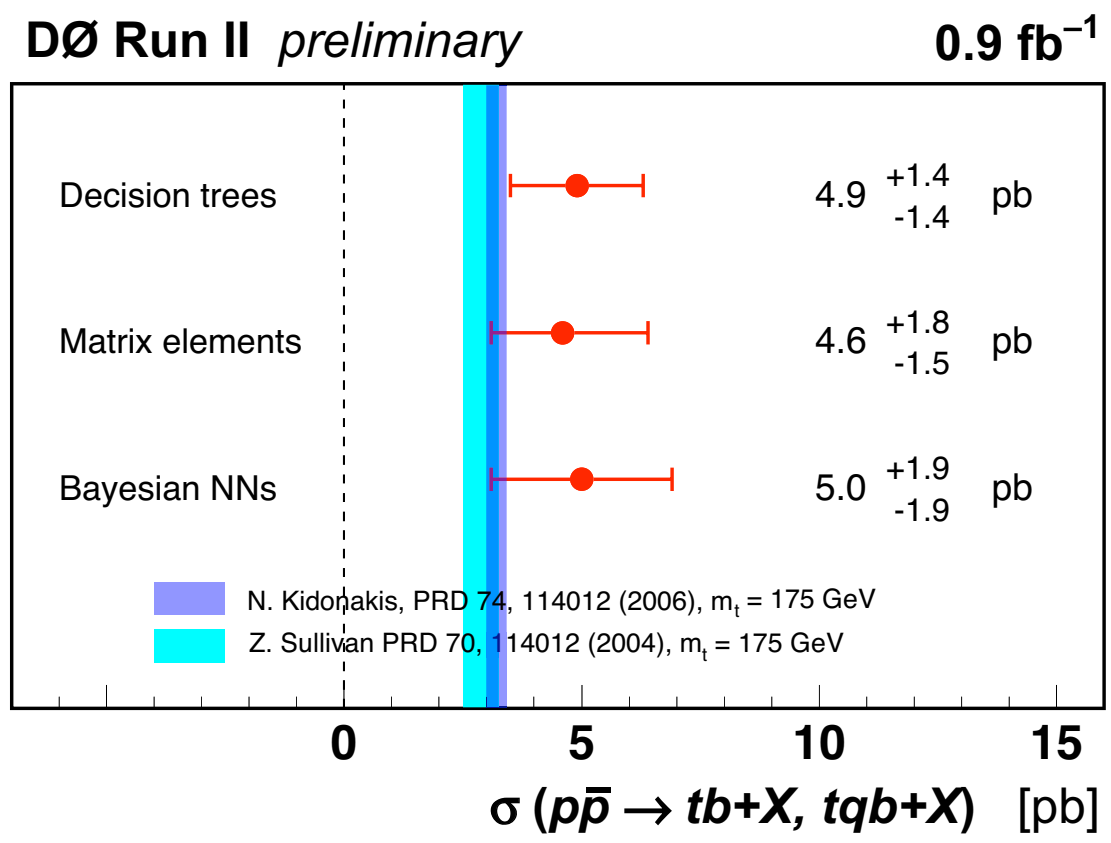

Figure 10.4: Measured Cross Sections from recent DØ measurements.

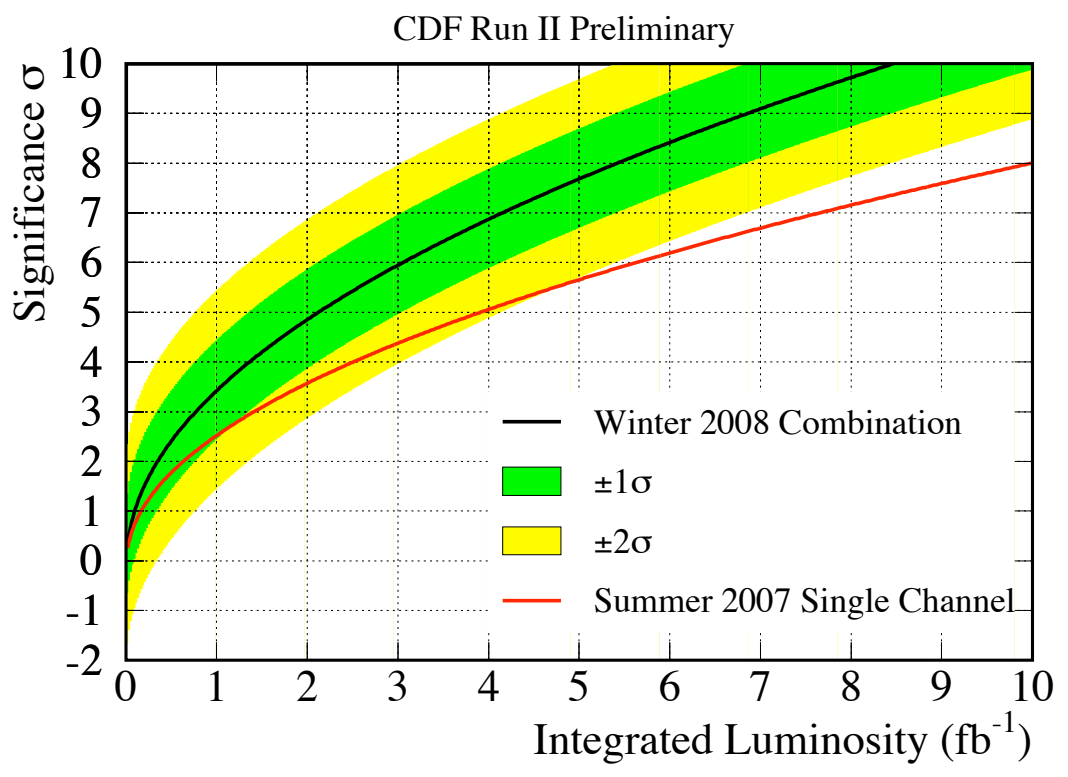

Figure 10.5: Predicted CDF combined significance 


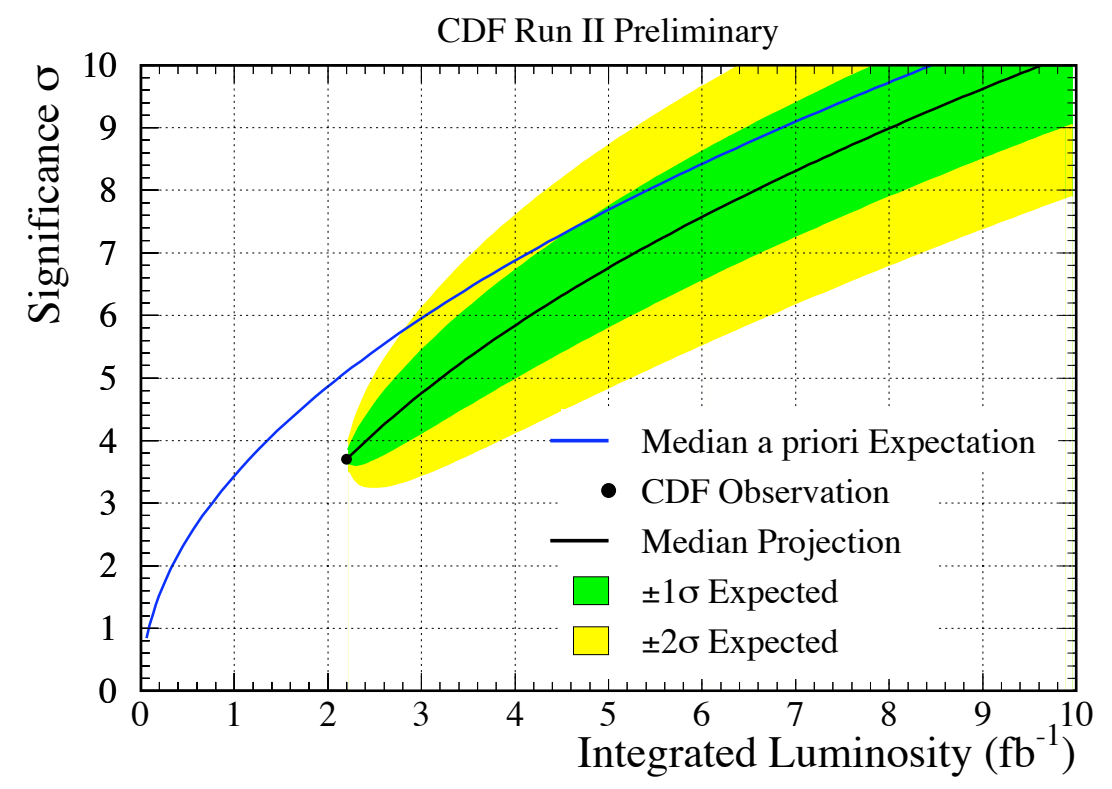

Figure 10.6: Predicted CDF combined significance extrapolated from current result. 


\section{A Mistag Matrix}

\section{A.1 Data Samples and Prescription}

The matrix was calculated with a $1.2 \mathrm{fb}^{-1}$ data set taken from inclusive jet triggers: JET20, JET50, JET70 and JET100. These triggers require a jet with a certain transverse momentum $\left(E_{T}\right)$, i.e. the JET20 trigger requires a Level 2 $E_{T}$ of $\geq 20 \mathrm{GeV}$, the JET50 trigger requires a Level $2 E_{T}$ of $\geq 50 \mathrm{GeV}$, the JET70 trigger requires a Level $2 E_{T}$ of $\geq 70 \mathrm{GeV}$, and the JET100 trigger requires a Level $2 E_{T}$ of $\geq 100 \mathrm{GeV}$. Good runs were defined by the good run list version 14. This is similar to the good run list described in Section 4.1.5, except it is good for an integrated luminosity of $1.2 \mathrm{fb}^{-1}$ rather than $2.2 \mathrm{fb}^{-1}$. Data from the all hadronic trigger ("multi-jet") was used for cross checks contributing to the systematic studies. At level 3, this trigger requires four jets, with at least one jet with $E_{T}>10 \mathrm{GeV}$. Finally, the JET20 and JET50 events were reweighted in order to account for changes in the fixed prescale values.

\section{A.2 Method}

The matrix was parameterized in six variables: measured transverse energy of the jet $\left(E_{T}\right)$, pseudo-rapidity of the jet $(\eta)$, SECVTX track multiplicity $\left(N_{t r k}\right)$, number of $\mathrm{Z}$ vertices, primary vertex $\mathrm{Z}$ position (PVZ), and the scaler sum of the transverse energy of all jets in the event $\left(\sum E_{T}\right)$. The binning of the tag matrices is shown in Table A.1.

The matrix was made with jets with a raw $E_{T}$ above $10 \mathrm{GeV}$, and $|\eta|$ less than 2.4. Additionally, a jet was considered taggable if it contained more than two SECVTX tracks. The positive and negative tag rates are defined as the ratio of tagged to taggable jets in each bin of the matrix. The formula for tag rates is given below, here $L_{x y}$ is the two dimensional decay length. The complete definition of positively and negatively tagged jets is given in Section 4.2.6.

$$
\begin{aligned}
& r_{+}^{i}=\frac{\text { Number of positively tagged jets (jets with } L_{x y}>0 \text { in bin i) }}{\text { Number of taggable jets in bin } \mathrm{i}} \\
& r_{-}^{i}=\frac{\text { Number of negatively tagged jets (jets with } L_{x y}<0 \text { in bin } \mathrm{i} \text { ) }}{\text { Number of taggable jets in bin } \mathrm{i}}
\end{aligned}
$$




\begin{tabular}{|c|c|c|c|c|c|}
\hline $\mathrm{E}_{T}(\mathrm{GeV})$ & $\mathrm{N}_{\text {trk }}$ & $\sum E_{T}$ & $|\eta|$ & \# Z vertices & $\mathrm{Z}(\mathrm{cm})$ \\
\hline $0-15$ & 2 & $0-80$ & $0-0.4$ & 1 & $-25--10$ \\
$15-22$ & 3 & $80-140$ & $0.4-0.8$ & 2 & $-10-10$ \\
$22-30$ & 4 & $140-220$ & $0.8-1.1$ & 3 & $10-25$ \\
$30-40$ & 5 & $>=220$ & $1.1-2.4$ & 4 & $>=25$ \\
$40-60$ & 6 & & & $>=6$ & \\
$60-90$ & 7 & & & & \\
$90-130$ & 8 & & & & \\
$>=130$ & 10 & & & & \\
& $>=13$ & & & & \\
\hline
\end{tabular}

Table A.1: Binning used in tag matrices. Shown are upper and lower bin edges.

\section{A.3 Cross Checks}

Several cross checks were performed on the tag matrix, which can be divided into the following categories: self-consistency, and predictivity.

\section{A.3.1 Self-Consistency}

A matrix created using even-numbered events was applied to odd-numbered events. The predicted and observed tag rates were compared for variables used to parametrize the matrix $\left(\mathrm{E}_{T}(\mathrm{GeV}), \mathrm{N}_{\text {trk }}, \sum E_{T},|\eta|\right.$, \# $\mathrm{Z}$ vertices, PZV). Figures A.1- A.3.1 show the results of this 'even-odd' cross check. Note that the matrices were made with re-weighted events (to account for changing prescales), and they were tested with non re-weighted events. This accounts for the small discrepancies in the plots. Mostly, these plots are made with the same binning as the mistag matrix, which means that other than the small discrepancies due to the event re-weighting these plots should all have exactly identical distributions for observed and predicted distributions. The exception is Figure A.3.1 which is shown with a finer binning than used in the actual matrix. For all plots, the upper portion of the plot shows the observed and predicted distributions for all jet data, as a function of either the positive or negative tag rate. The black line indicates the observed distribution, and the red dashed line indicates the distribution predicted by the mistag matrix. In the lower portion of the plot, the 'delta tag rate' given by (observed-predicted)/observed is plotted. 

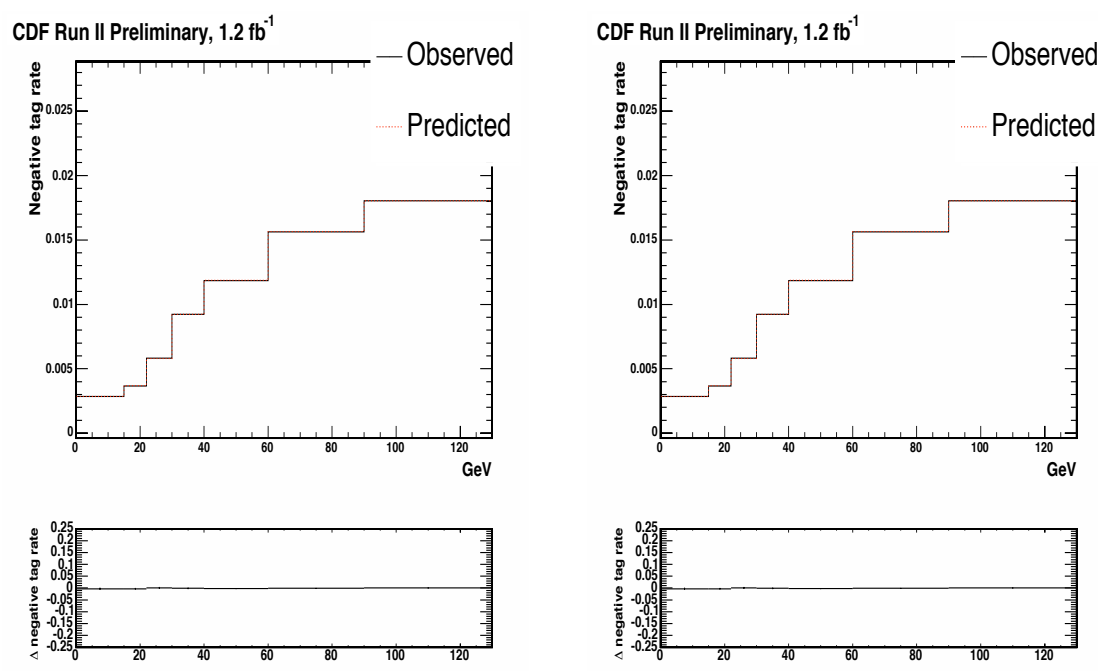

Figure A.1: Observed and predicted tag rates as a function of $E_{T}$. The left plot was created using the negative tag matrix and the right plot was created using the positive tag matrix.
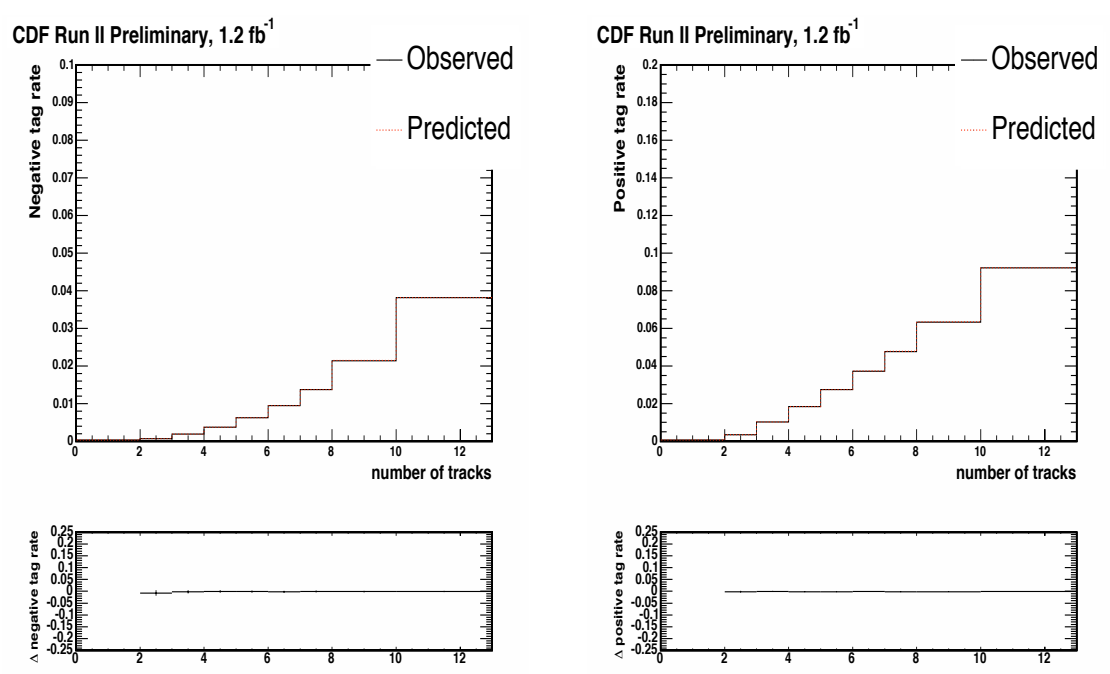

Figure A.2: Observed and predicted tag rates as a function of the number of good tracks. The left plot was created using the negative tag matrix and the right plot was created using the positive tag matrix. 

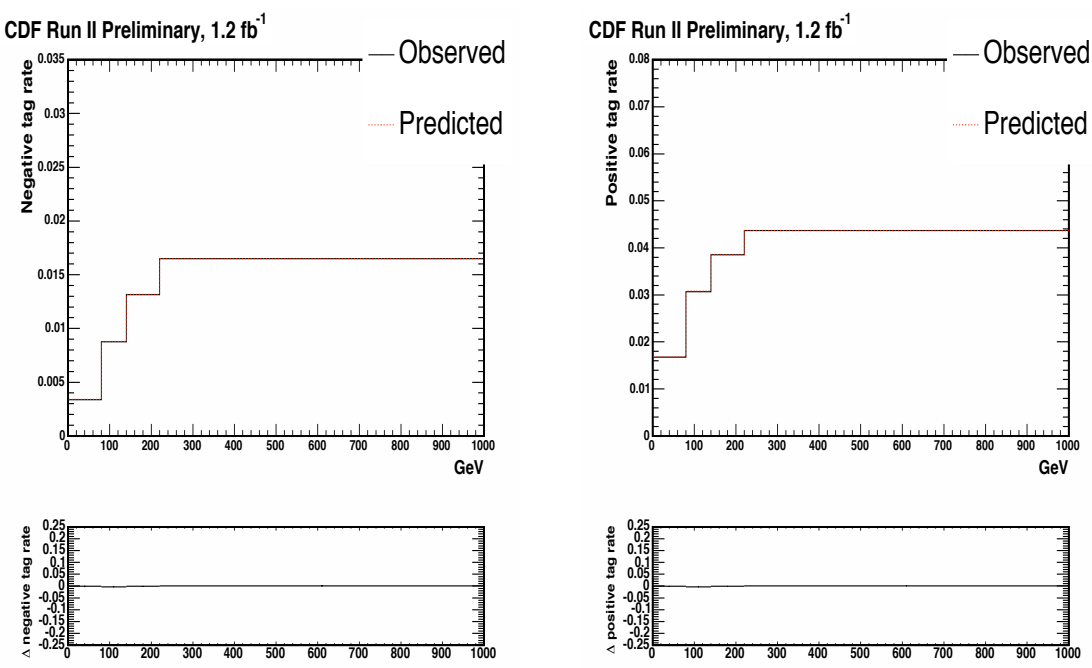

Figure A.3: Observed and predicted tag rates as a function of $\sum E_{T}$. The left plot was created using the negative tag matrix and the right plot was created using the positive tag matrix.
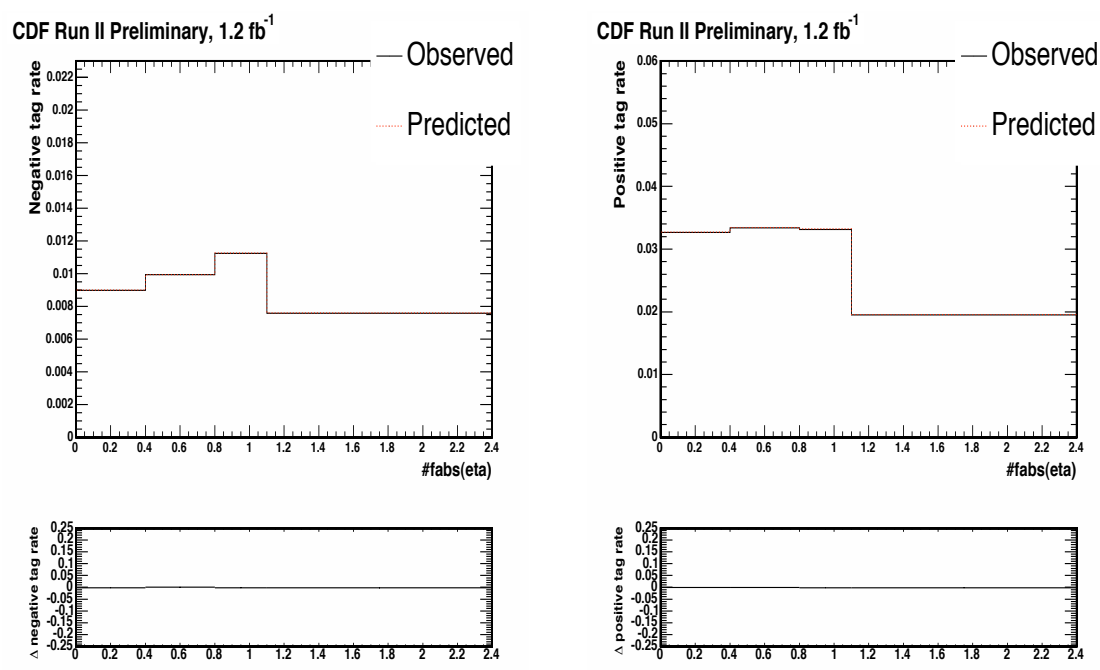

Figure A.4: Observed and predicted tag rates as a function of $\eta$. The left plot was created using the negative tag matrix and the right plot was created using the positive tag matrix. 

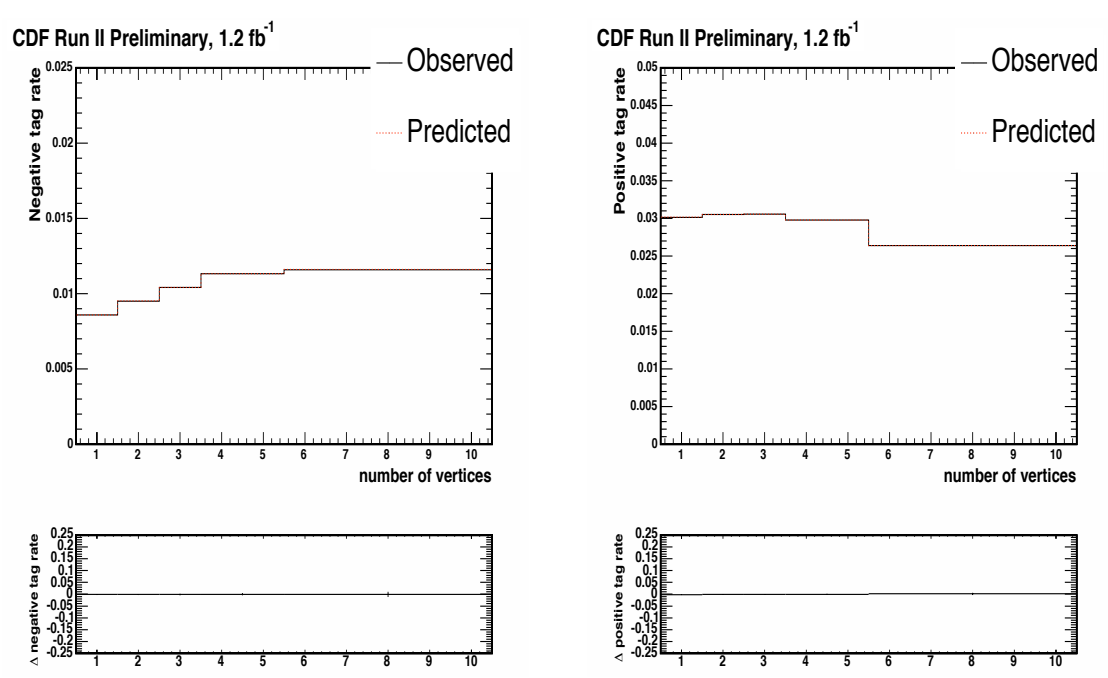

Figure A.5: Observed and predicted tag rates as a function of the number of primary verticies in the event. The left plot was created using the negative tag matrix and the right plot was created using the positive tag matrix.
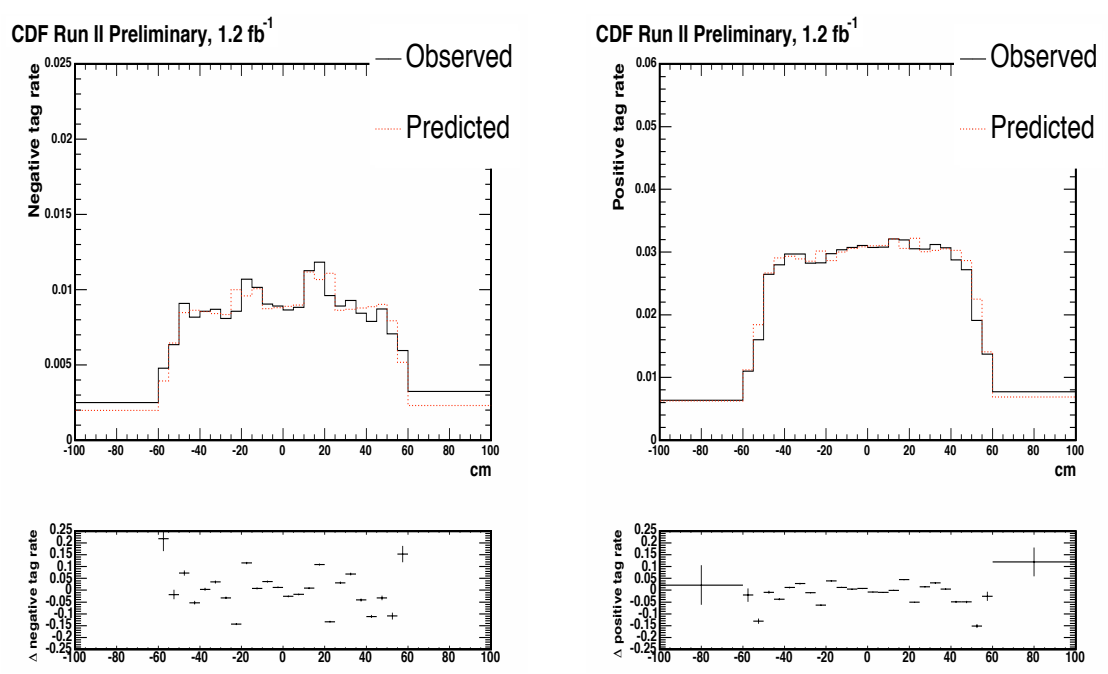

Figure A.6: Observed and predicted tag rates as a function of the $z$ position of the primary vertex is shown. Note that the binning shown in this plot is much finer than the matrix binning, hence the discrepancies in observed and predicted rates. The left plot was created using the negative tag matrix and the right plot was created using the positive tag matrix. 


\section{A.3.2 Predictivity}

The matrix was used to predict the mistag rates as functions of several variables not included in the matrix parameterization. These variables include

- Run Number (a function of time)

- Instantaneous Luminosity

- Bunch Luminosity

- Total number of jets in the event

- Primary vertex $\mathrm{X}$ error

- Primary vertex Y errors

- Total number of tracks in the event

- $\phi$ of the Jet

Figures A.7-A.14 show the results of this cross check. These plots do not show the nearly perfect agreement between observed and predicted tag rates seen in the self-consistency variables. This is to be expected as the kinematic variables can be highly dependent on event topology. However, the overall tag rate is well predicted by the matrix.
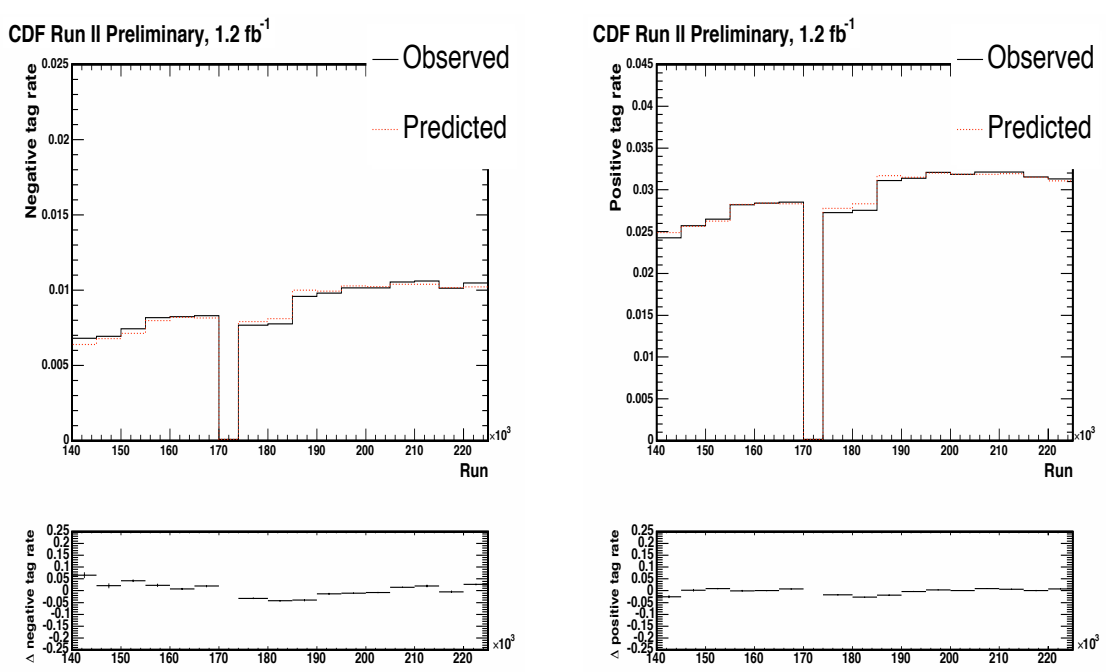

Figure A.7: Observed and predicted tag rates as a function of the run number. The left plot was created using the negative tag matrix and the right plot was created using the positive tag matrix. 

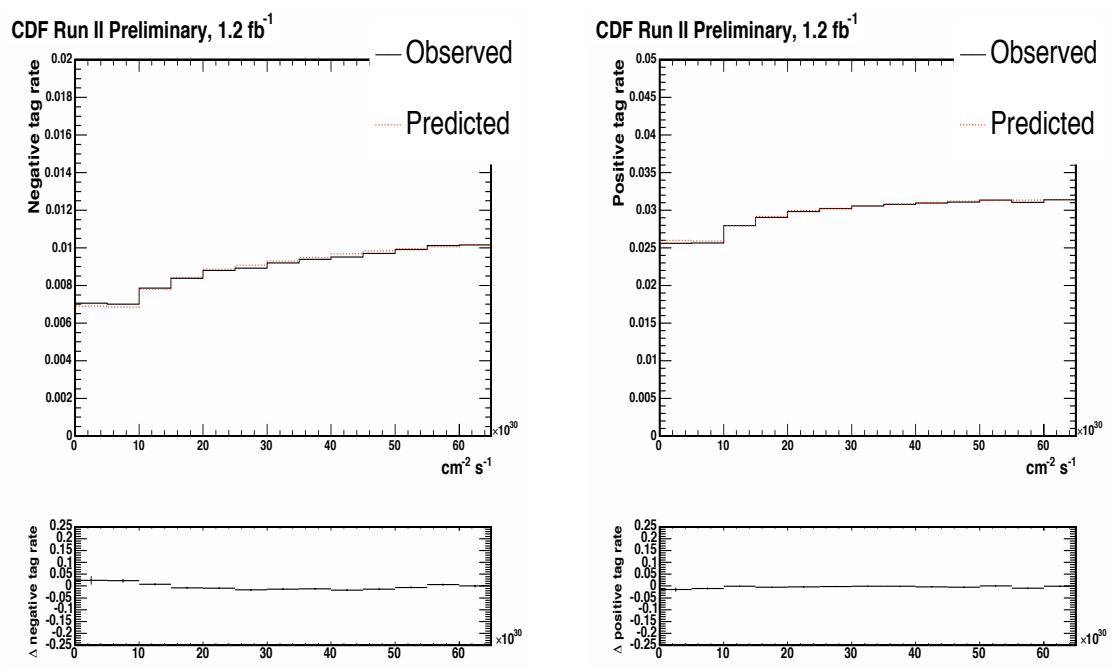

Figure A.8: Observed and predicted tag rates as a function of the luminosity of the event. The left plot was created using the negative tag matrix and the right plot was created using the positive tag matrix.
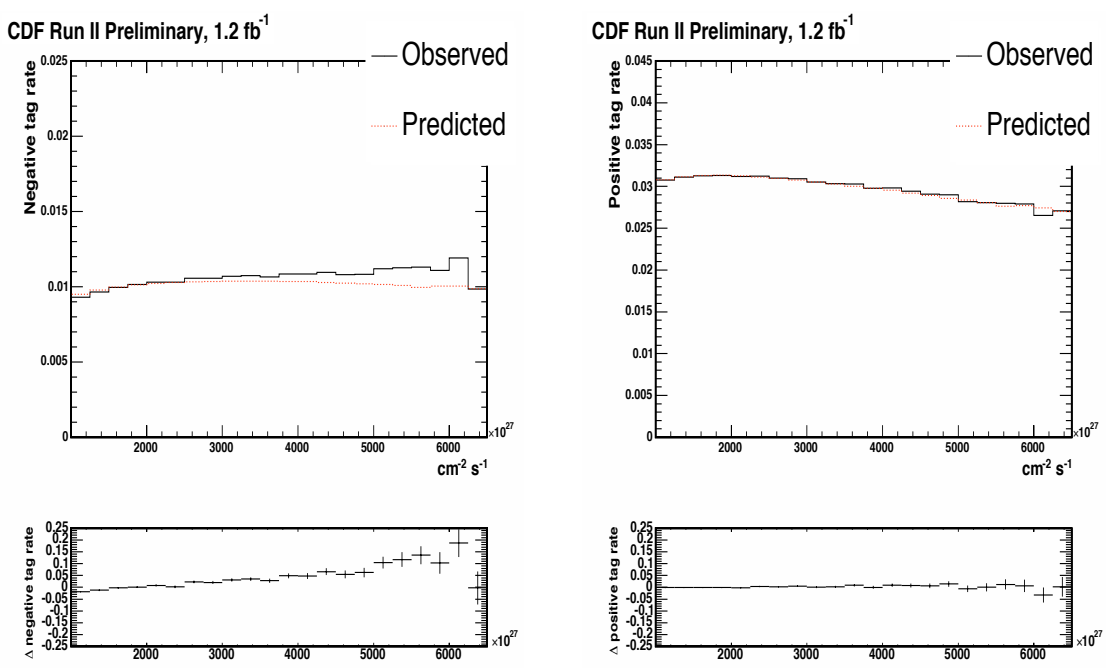

Figure A.9: Observed and predicted tag rates as a function of the bunch luminosity. The left plot was created using the negative tag matrix and the right plot was created using the positive tag matrix. 

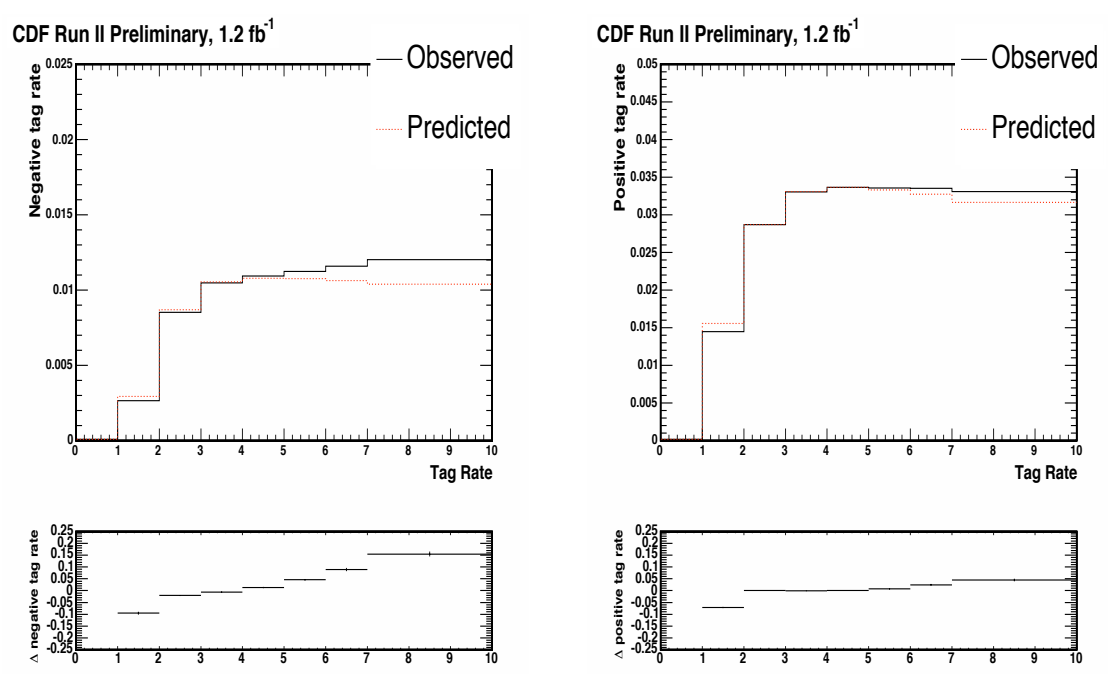

Figure A.10: Observed and predicted tag rates as a function of the number of jets in the event. The left plot was created using the negative tag matrix and the right plot was created using the positive tag matrix.
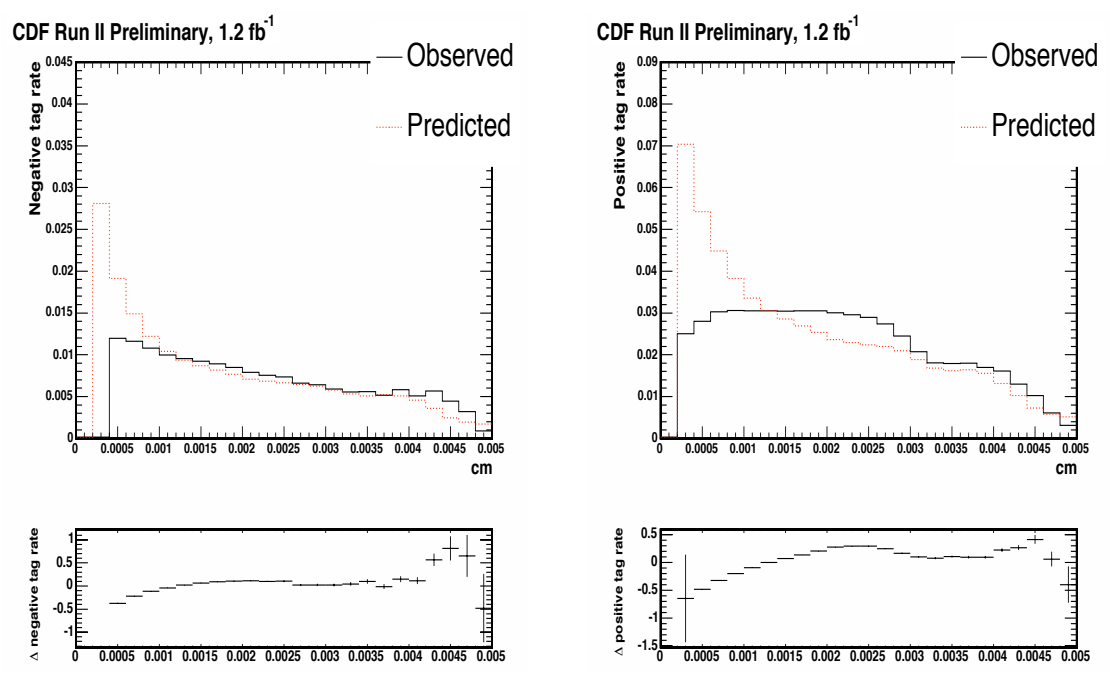

Figure A.11: Observed and predicted tag rates as a function of error associated with the primary vertex position in the y direction. The left plot was created using the negative tag matrix and the right plot was created using the positive tag matrix. 

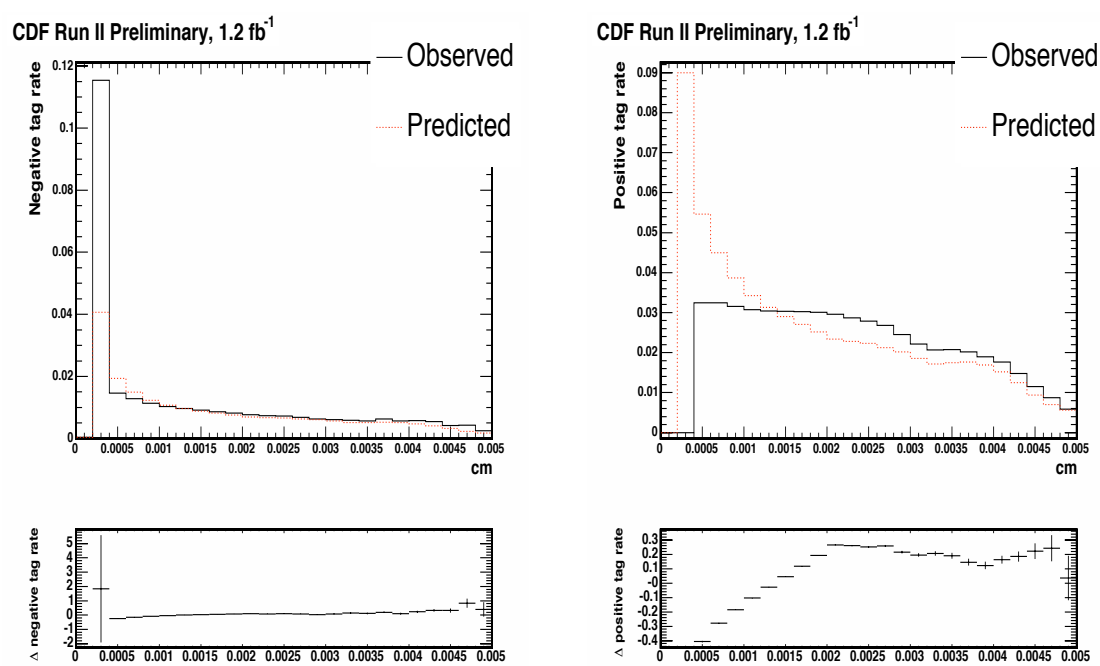

Figure A.12: Observed and predicted tag rates as a function of error associated with the primary vertex position in the $\mathrm{x}$ direction. The left plot was created using the negative tag matrix and the right plot was created using the positive tag matrix.
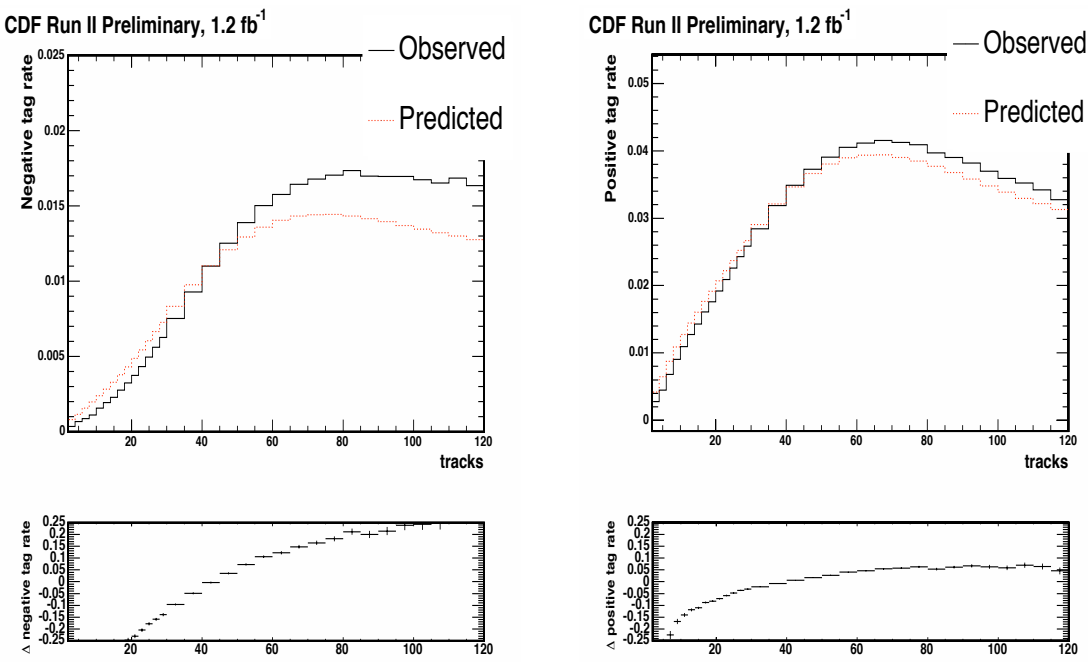

Figure A.13: Observed and predicted tag rates as a function of the total number of tracks in the event. The left plot was created using the negative tag matrix and the right plot was created using the positive tag matrix. 

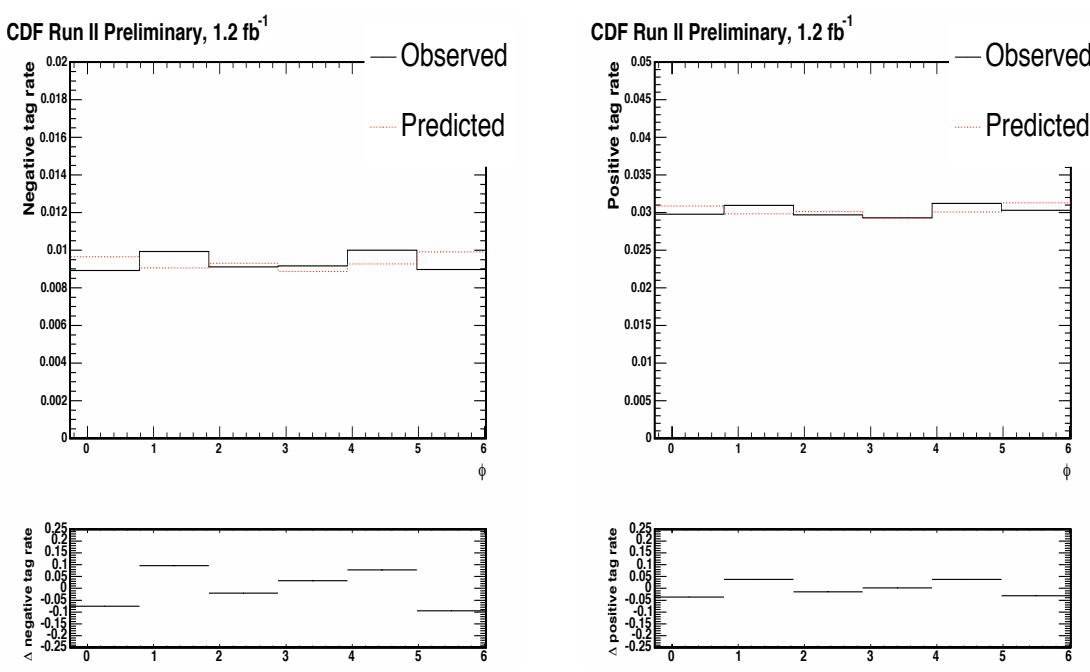

Figure A.14: Observed and predicted tag rates as a function of $\phi$. The left plot was created using the negative tag matrix and the right plot was created using the positive tag matrix. 


\section{A.4 Mistag Rate Systematics}

Several rate systematics associated with the mistag matrix were calculated. These were combined to form a total rate uncertainty on the mistag prediction. In the likelihood analysis, this uncertainty was folded into the event selection rate uncertainty, and a separate systematic for the mistag shape was assessed. See Section 8.1 for full details.

In the calculation of the mistag rate systematic, three sources of systematic error were considered: sample dependence, $\sum E_{T}$ dependence, and trigger jet bias. Systematics were calculated by measuring the difference between observed and predicted rates when the mistag matrix was run over various samples. Table A.2 summarizes the results of all systematic tests.

For the sample dependence systematic, the matrix was tested using events collected in various subsamples, including the JET20, JET50, JET70, JET100 and multi-jet samples. This systematic was designed to quantify the error associated with using a matrix created with one data trigger on a sample from a separate data trigger. The jet samples were tested with 'odd' events using 'even' matrices while the multi-jet sample was tested using all events with the matrix created with all events. The average tag rates for the cross check samples is shown in Tables A.2. The largest deviation between the total observed and predicted tag rates was taken as the systematic error. For instance, for the positive matrix the sample dependence check with the largest deviation from unity is the multi-jet check, with a Predicted/Observed ratio of 1.037. This leads to a $3.7 \%$ sample dependence systematic for the positive matrix.

For the $\sum E_{T}$ systematic, a cross check of the $\sum E_{T}$ variable was implemented by varying the event $\sum E_{T}$ in the inclusive jet samples. This systematic quantifies the uncertainty associated with the $\sum E_{T}$ variable due to the possible loss of a jet. The systematic was implemented by adding the mean jet energy in each event to the $\sum E_{T}$ variable. Half the largest deviation from unity was taken as a systematic. Again, for the positive tight matrix, the Predicted/Observed ratio is 0.955 giving a systematic of $2.2 \%((1-0.955) / 2)$.

The trigger bias systematic was calculated by examining 'trigger' jets. This systematic is designed to account for difference in the tag rates between jets which trigger the event and other jets observed in earlier data sets. This difference is due in part to trigger bias in the $\eta$ and phi of trigger and non-trigger jets. 'Trigger' jets are defined as the jets closest to the Level 2 calorimeter cluster that fired the jet trigger. The trigger bias systematic is taken as the largest deviation from unity resulting from this check. For the positive matrix, the Predicted/Observed ratio is 0.966 giving a systematic of $3.4 \%$

A summary of the total tag rates for these checks is given in table A.2. The total systematic uncertainty is given in table A.3. The full mistag matrix systematics used in this analysis are shown in Table A.3. 


\begin{tabular}{|c|c|c|c|}
\hline \multicolumn{4}{|c|}{ Tight Matrix } \\
\hline Check & Pos Observed & Pos Predicted & Pos Ratio \\
\hline \multicolumn{4}{|c|}{ Sample Dependence } \\
\hline Even-Odd, All Jet Data & $0.014240 \pm 0.000008$ & $0.014264 \pm 0.000008$ & $0.998 \pm 0.001$ \\
\hline Even-Odd, Jet20 & $0.006012 \pm 0.000008$ & $0.006155 \pm 0.000008$ & $0.977 \pm 0.002$ \\
\hline Even-Odd, Jet50 & $0.014439 \pm 0.000017$ & $0.014644 \pm 0.000017$ & $0.986 \pm 0.002$ \\
\hline Even-Odd, Jet70 & $0.019691 \pm 0.000021$ & $0.019750 \pm 0.000021$ & $0.997 \pm 0.002$ \\
\hline Even-Odd, Jet100 & $0.023995 \pm 0.000022$ & $0.023593 \pm 0.000022$ & $1.017 \pm 0.001$ \\
\hline Multi-Jet Sample & $0.014979 \pm 0.000015$ & $0.014448 \pm 0.000015$ & $1.037 \pm 0.002$ \\
\hline \multicolumn{4}{|c|}{$\sum E_{T}$ systematic } \\
\hline Scaling $\sum E_{T}$ up & $0.014240 \pm 0.000008$ & $0.014911 \pm 0.000008$ & $0.955 \pm 0.001$ \\
\hline \multicolumn{4}{|c|}{ Trigger Bias Systematic } \\
\hline Trigger Jets & $0.012691 \pm 0.000012$ & $0.013134 \pm 0.000012$ & $0.966 \pm 0.001$ \\
\hline Check & $\overline{\text { Neg Observed }}$ & $\overline{\overline{N e g} \text { Predicted }}$ & $\overline{\text { Neg Ratio }}$ \\
\hline \multicolumn{4}{|c|}{ Sample Dependence } \\
\hline Even-Odd, All Jet Data, & $0.004398 \pm 0.000004$ & $0.004404 \pm 0.000004$ & $0.998 \pm 0.001$ \\
\hline Even-Odd, Jet20 & $0.001176 \pm 0.000004$ & $0.001225 \pm 0.000004$ & $0.961 \pm 0.004$ \\
\hline Even-Odd, Jet50 & $0.004060 \pm 0.000009$ & $0.004131 \pm 0.000009$ & $0.983 \pm 0.003$ \\
\hline Even-Odd,Jet70 & $0.006355 \pm 0.000012$ & $0.006414 \pm 0.000012$ & $0.991 \pm 0.003$ \\
\hline Even-Odd,Jet100 & $0.008789 \pm 0.000013$ & $0.008612 \pm 0.000013$ & $1.021 \pm 0.002$ \\
\hline Multi-Jet Sample & $0.004149 \pm 0.000008$ & $0.004262 \pm 0.000008$ & $0.973 \pm 0.003$ \\
\hline \multicolumn{4}{|c|}{$\sum E_{T}$ systematic } \\
\hline Scaling $\sum E_{T}$ up & $0.004398 \pm 0.000004$ & $0.004705 \pm 0.000005$ & $0.935 \pm 0.001$ \\
\hline \multicolumn{4}{|c|}{ Trigger Bias Systematic } \\
\hline Trigger Jets & $0.003933 \pm 0.000007$ & $0.004053 \pm 0.000007$ & $0.970 \pm 0.002$ \\
\hline
\end{tabular}

Table A.2: Cross checks for tight tag matrices. The systematics were taken from the elements checks shown in bold. The sample dependence and trigger jet systematics are given by the largest deviation from unity, while the $\sum E_{T}$ scaling systematic is taken as half the largest deviation from unity. The errors were calculated from the statistics of the test sample and the mistag matrix itself.

\begin{tabular}{|l|c|c|}
\hline & Positive & Negative \\
\hline Sample & $3.7 \%$ & $3.9 \%$ \\
$\sum E_{T}$ & $2.2 \%$ & $3.3 \%$ \\
Trigger & $3.4 \%$ & $3.0 \%$ \\
\hline Total for $1.2 \mathrm{fb}^{-} 1$ matrix & $5.5 \%$ & $5.9 \%$ \\
\hline
\end{tabular}

Table A.3: Summary of Systematic Uncertainties for the Mistag Matrix. 


\section{Shape Systematic Plots}

The shape systematics used are given below for the 2-jet and 3-jet bins. For each plot, the upper portion has the central shape in the solid line, the positively varied shape in the dotted line, and the negatively varied shape in the dashed line. For one sided systematics, the varied distribution is given by the dashed line. In the lower portion the relative errors are plotted, given by (variedcentral)/central. For the relative error plots, a median smoothing algorithm with a window of five bins is applied as described in Section 8.1. Here the positive variation is plotted with the diamonds and the negative variation is plotted with the squares. For one sided systematics, the varied sample is given by the squares.

\section{B.1 Shape Systematic Plots, 2-jet Bin}
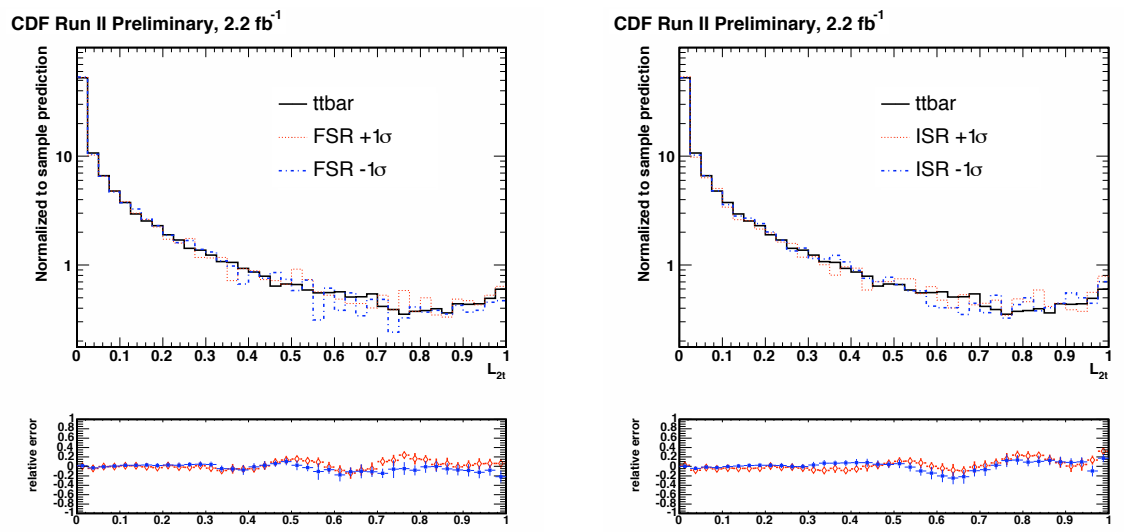

Figure B.1: FSR and ISR shape systematics, for the $t \bar{t}$ sample. 

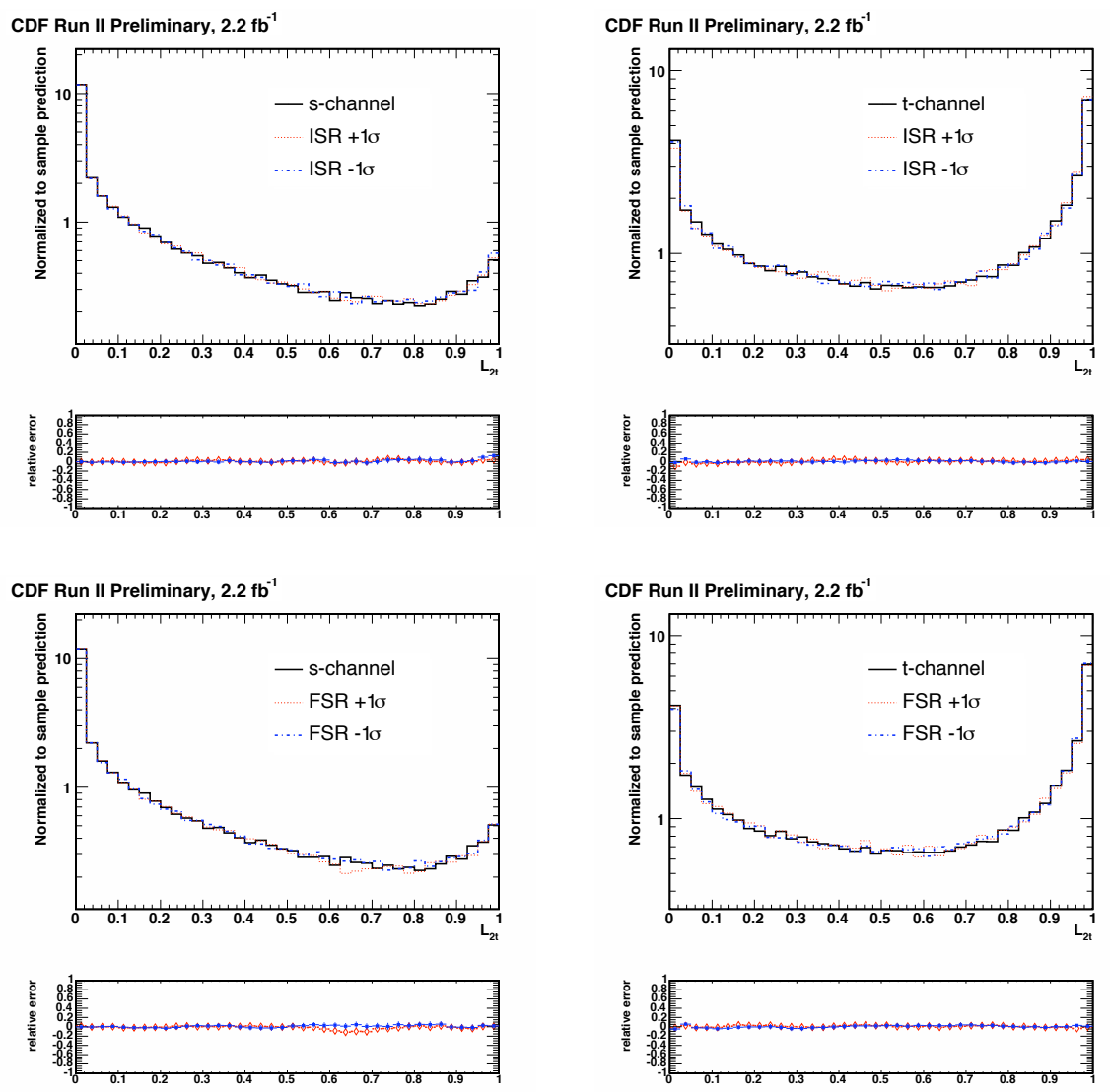

Figure B.2: FSR and ISR shape systematics for the $s$-channel and $t$-channel signal samples. 

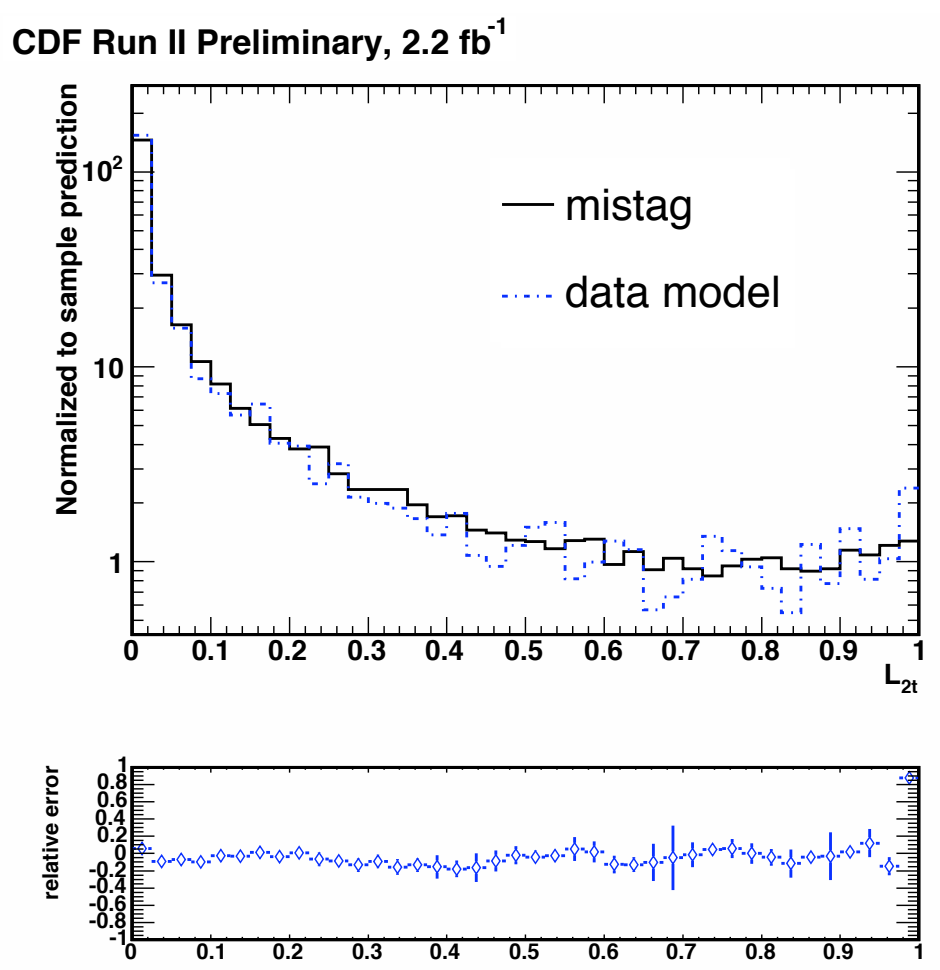

Figure B.3: Comparison between mistag model and pretag data weighted using the mistag matrix in the two-jet bin. 


\section{CDF Run II Preliminary, $2.2 \mathrm{fb}^{-1}$}
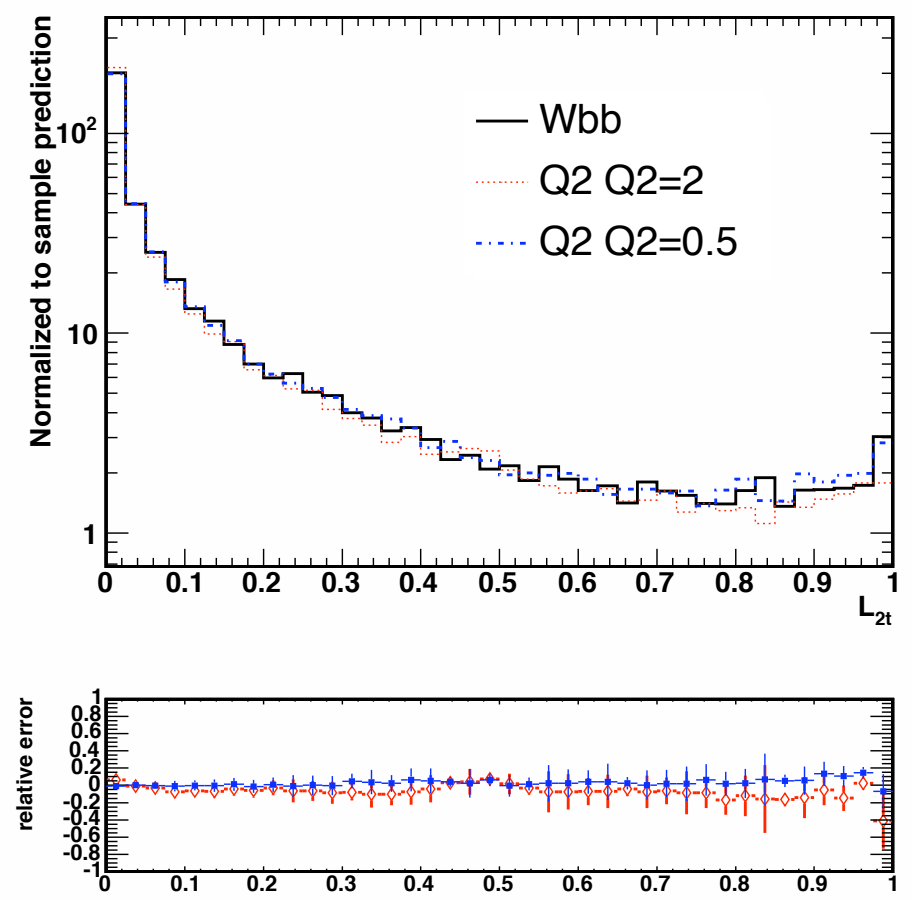

Figure B.4: $Q^{2}$ Shape systematics for the Wbb sample in the two-jet bin.
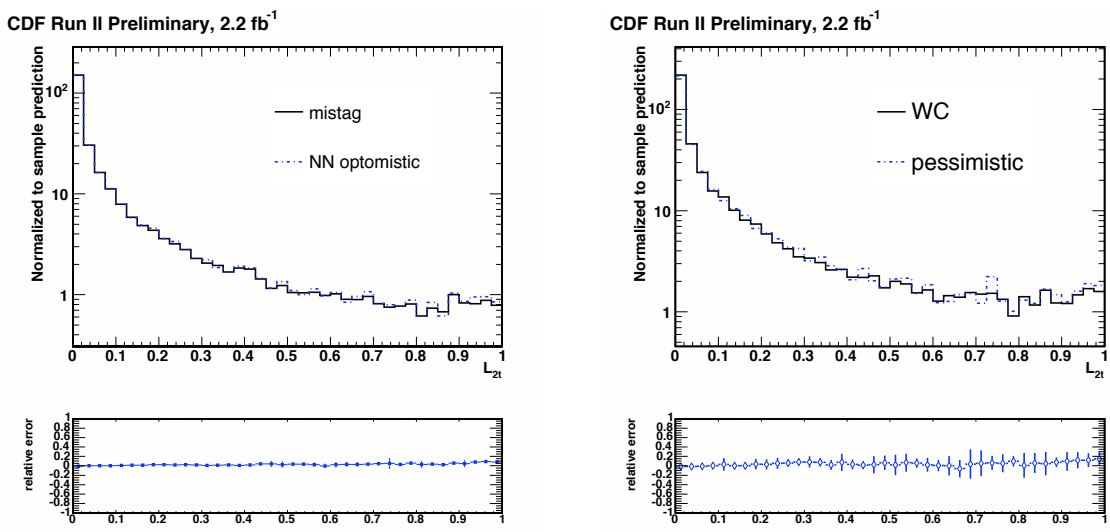

Figure B.5: NN B-Tag Shape systematics for mistag and $W c$ predictions in the two-jet bin. 
CDF Run II Preliminary, $2.2 \mathrm{fb}^{-1}$
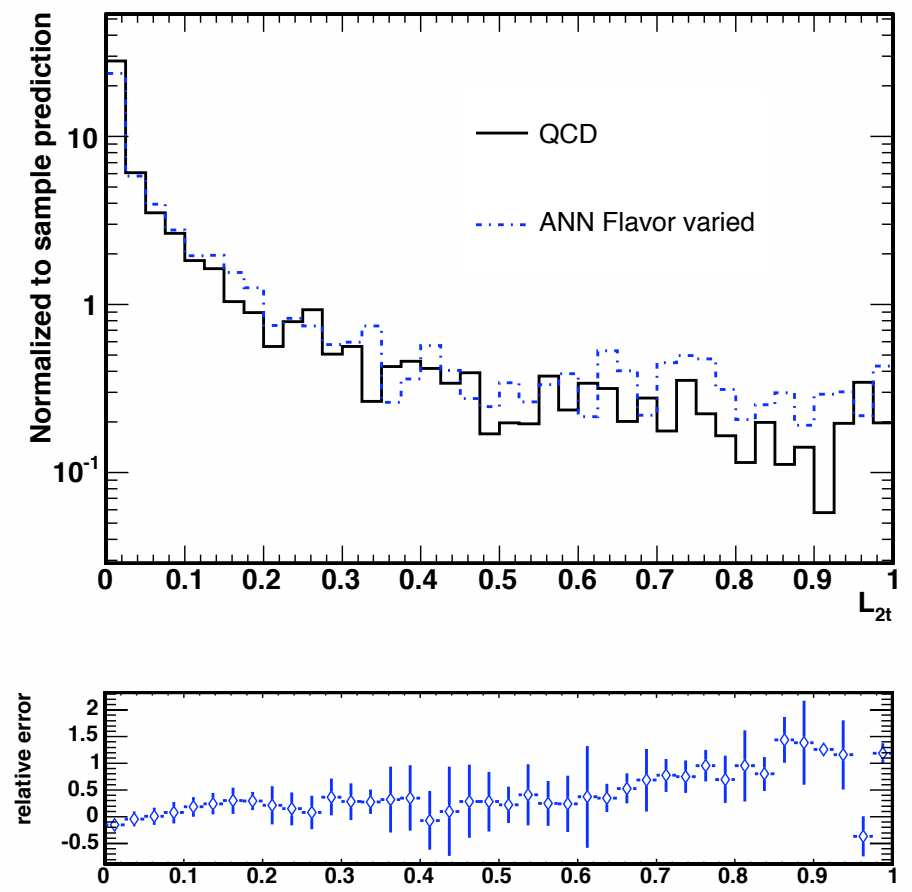

Figure B.6: Non- $W$ flavor modeling shape systematic in the two-jet bin. Shown is the ratio of (varied-central)/central. The central value of the non-W flavor composition is estimated to be $45 \% \mathrm{~b}, 40 \%$ charm, and $15 \%$ light flavor. The systematic variation is $60 \% \mathrm{~b}, 30 \%$ charm, and $10 \%$ light flavor.
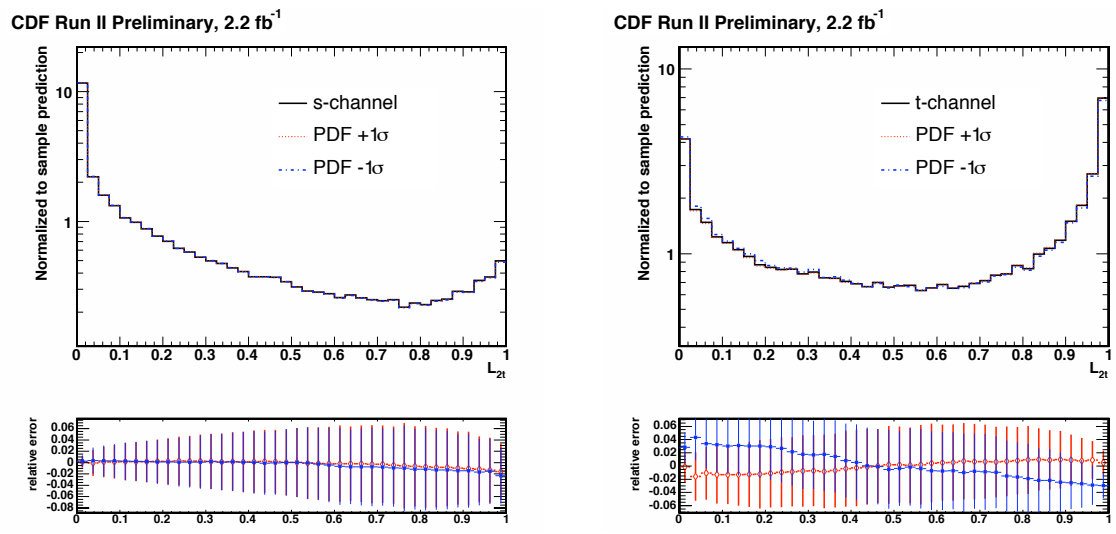

Figure B.7: PDF shape systematics for the $t$-channel and $s$-channel signal predictions in the two-jet bin. 

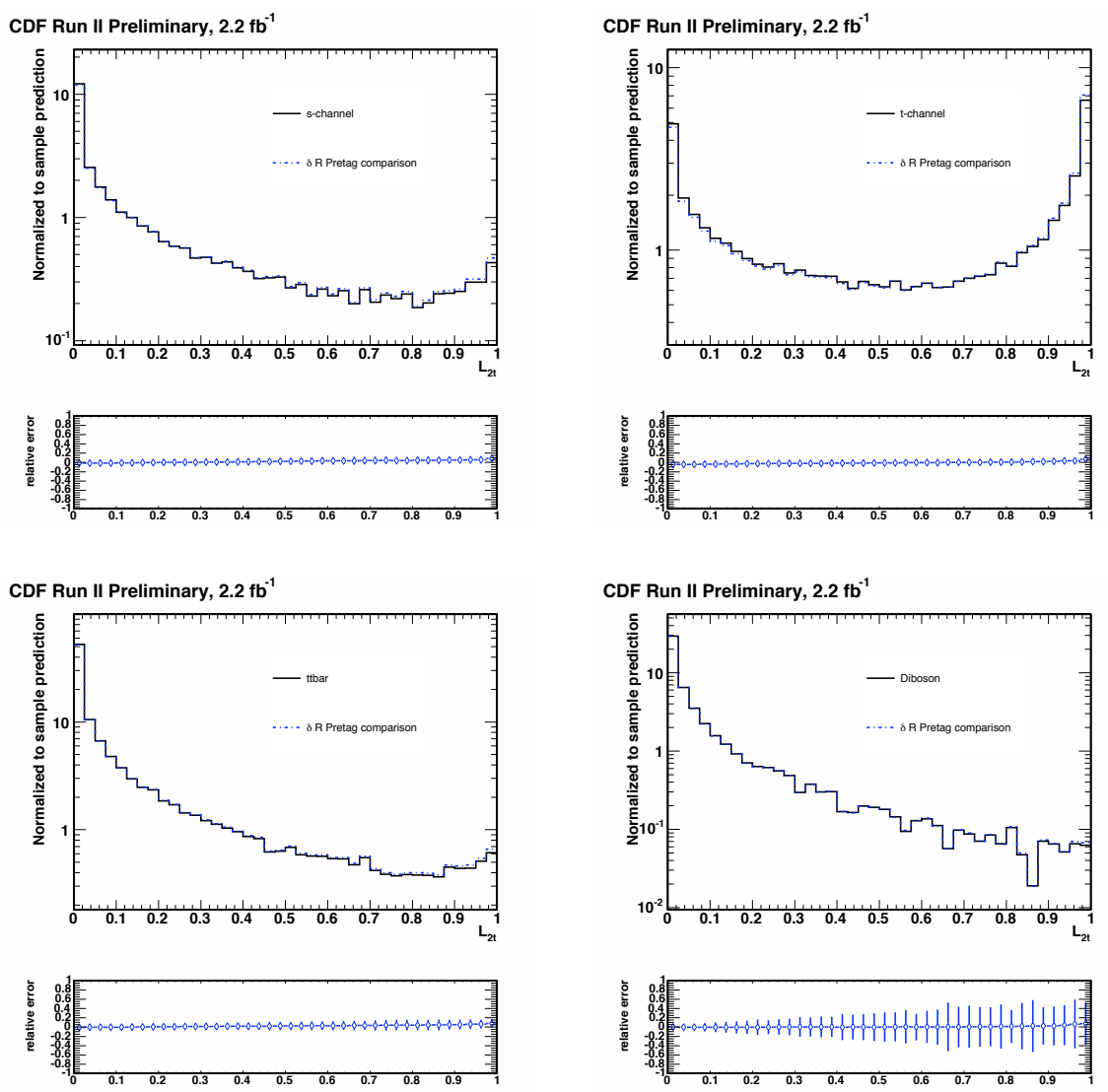

Figure B.8: Shape systematics induced by reweighting $\Delta R$ of the two jets for the $s$-channel signal, the $t$-channel signal, and the $t \bar{t}$ and diboson backgrounds in the two-jet bin. 

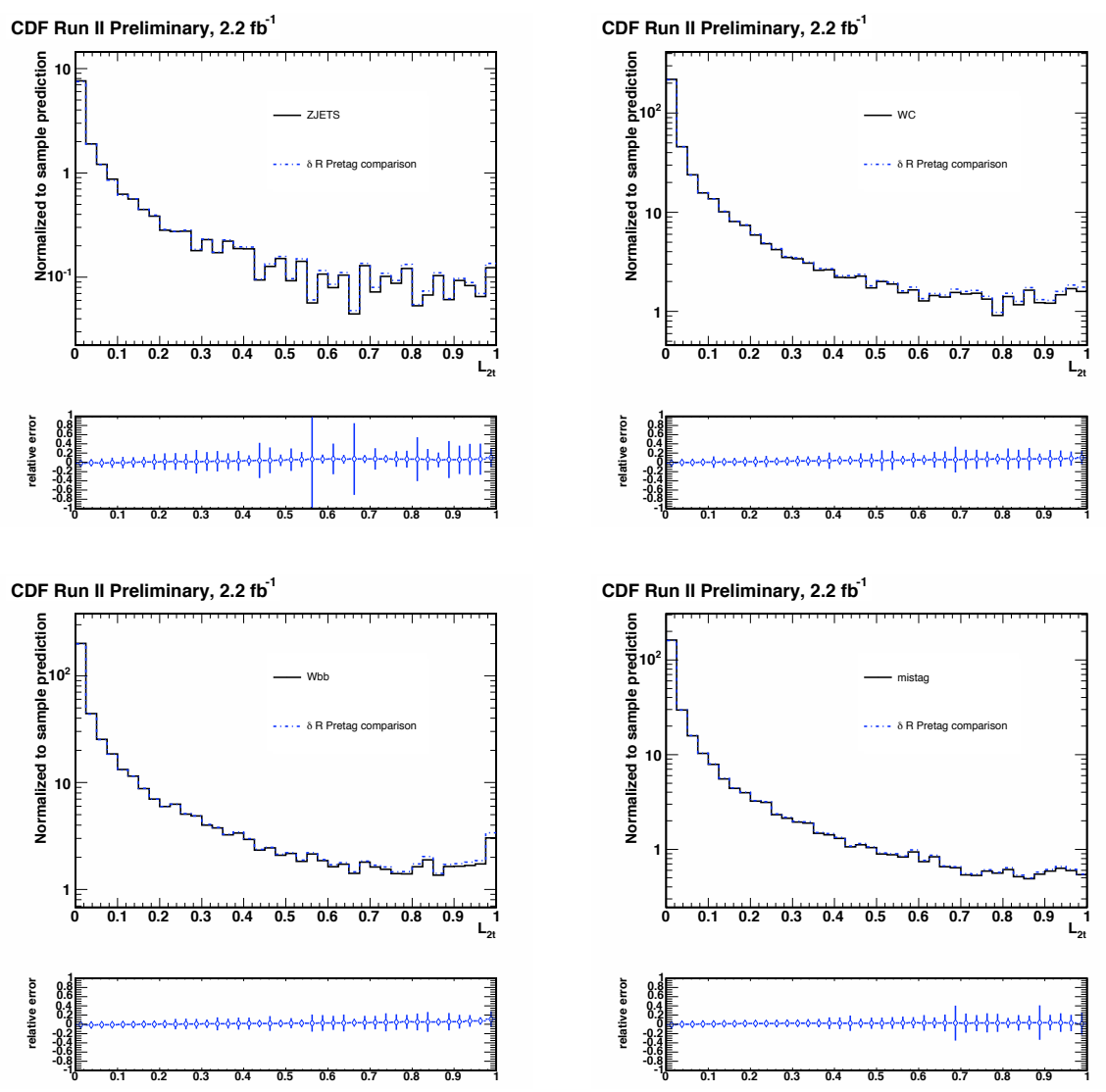

Figure B.9: Shape systematics induced by reweighting $\Delta R$ of the two jets for the $Z+$ jets, $W c / W c c, W b b$ and mistag backgrounds in the two-jet bin. 

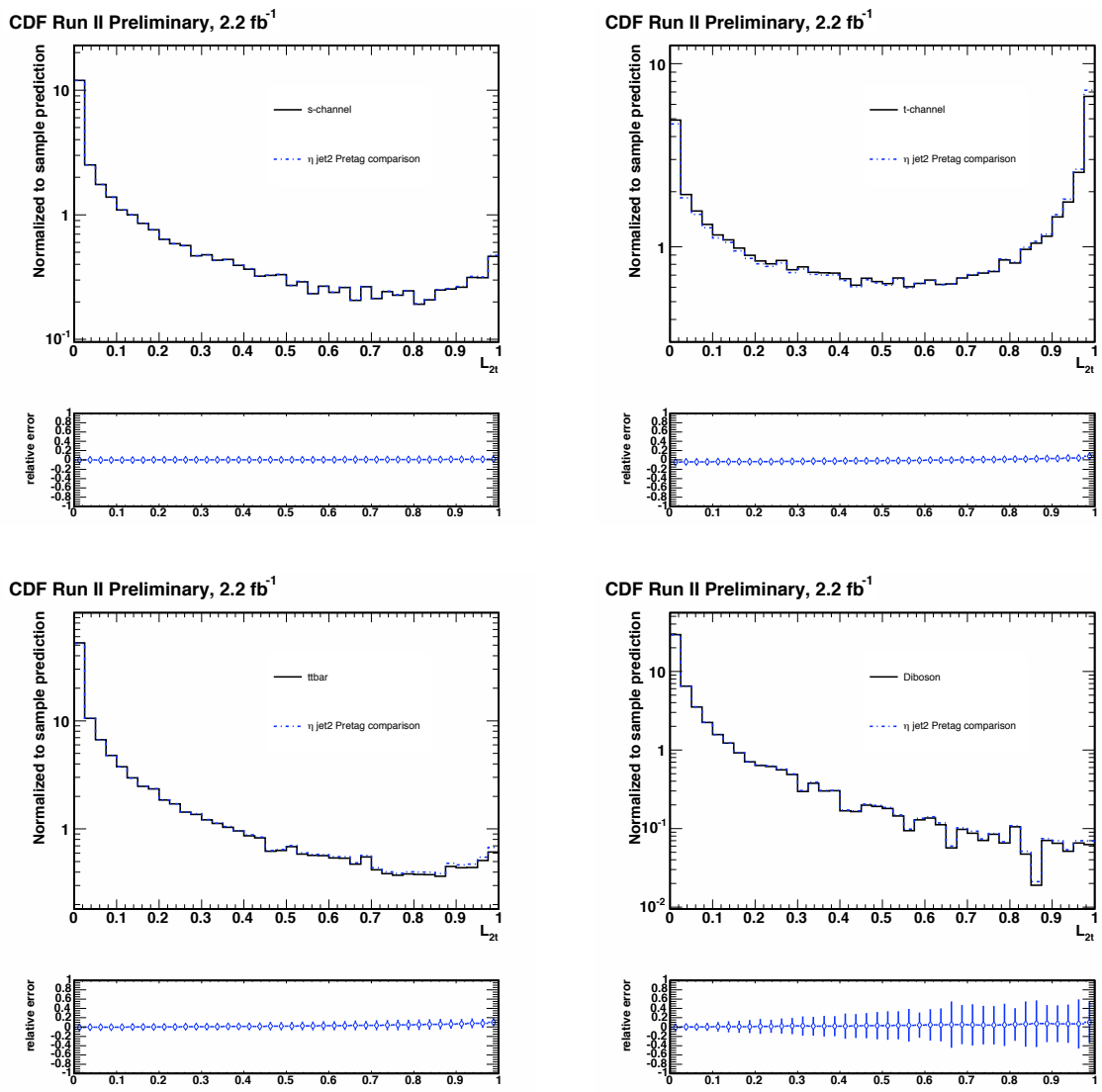

Figure B.10: Shape systematics induced by reweighting $\eta_{j 2}$ for the $s$-channel signal, the $t$-channel signal, and the $t \bar{t}$ and diboson backgrounds in the two-jet bin. 

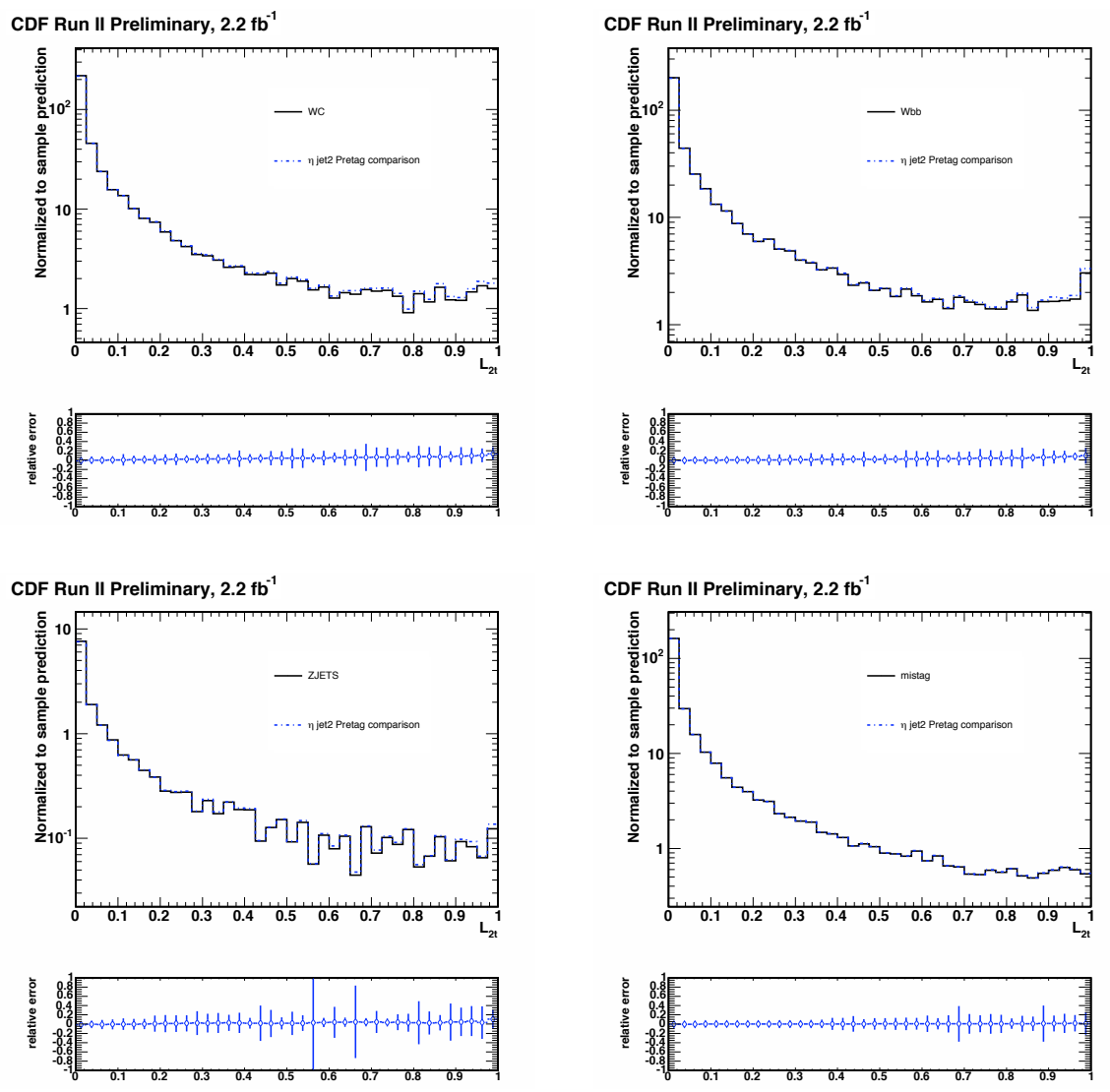

Figure B.11: Shape systematics induced by reweighting $\eta_{j 2}$ of the two jets for the $Z+$ jets, $W c / W c c, W b b$ and mistag backgrounds in the two-jet bin. 

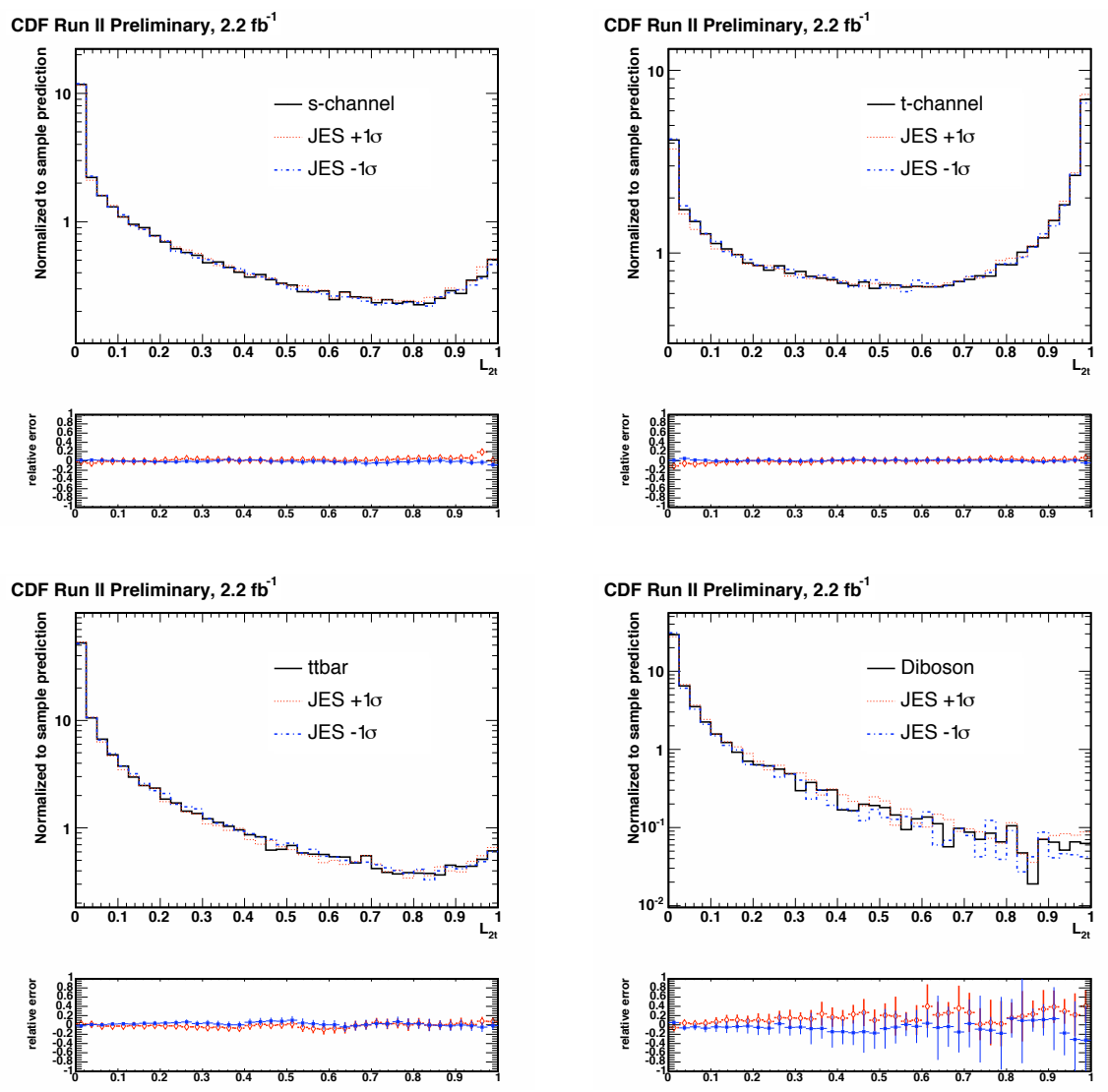

Figure B.12: Jet Energy Scale shape systematics for the $s$-channel signal, the $t$-channel signal, and the $t \bar{t}$ and diboson backgrounds in the two-jet bin. 

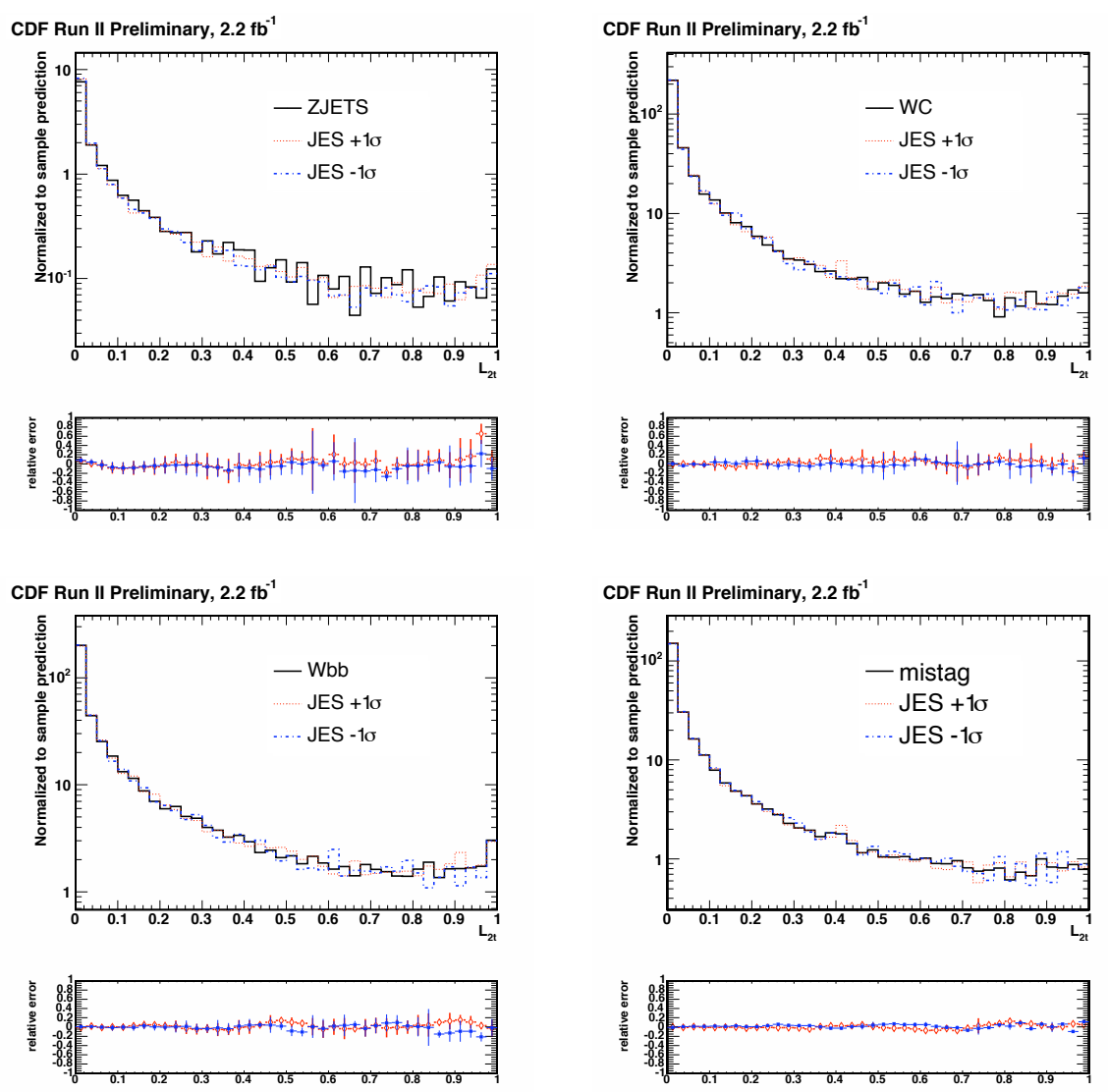

Figure B.13: Jet Energy Scale shape systematics for $Z+$ jets, $W c / W c c, W b b$ and mistag backgrounds in the two-jet bin. 

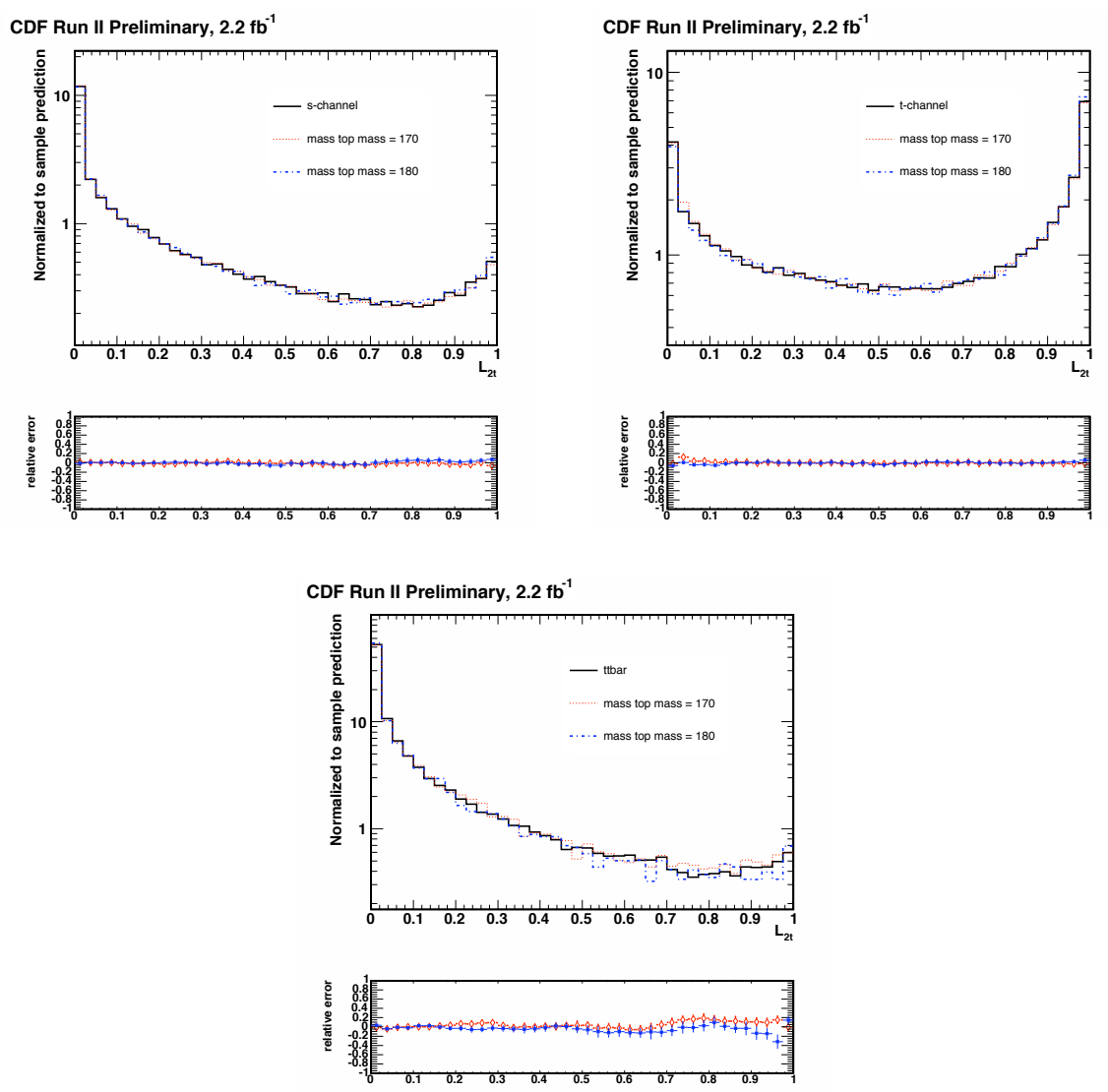

Figure B.14: Top mass shape systematics for $t$-channel, and $s$-channel signal samples and $t \bar{t}$ background in the two-jet bin. 


\section{B.2 Shape Systematic Plots, 3-jet Bin}
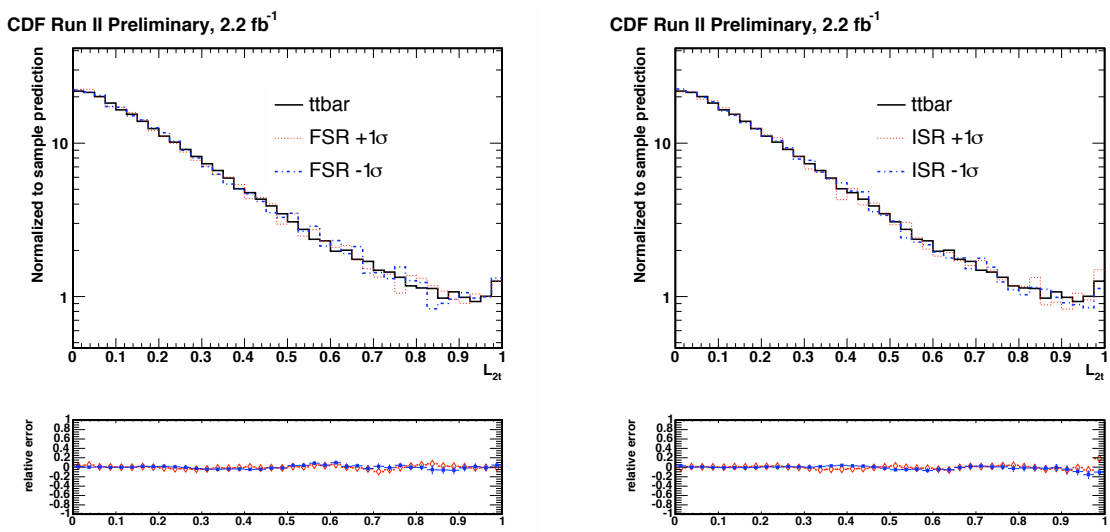

Figure B.15: FSR and ISR shape systematics, for the $t \bar{t}$ sample in the three-jet bin. 

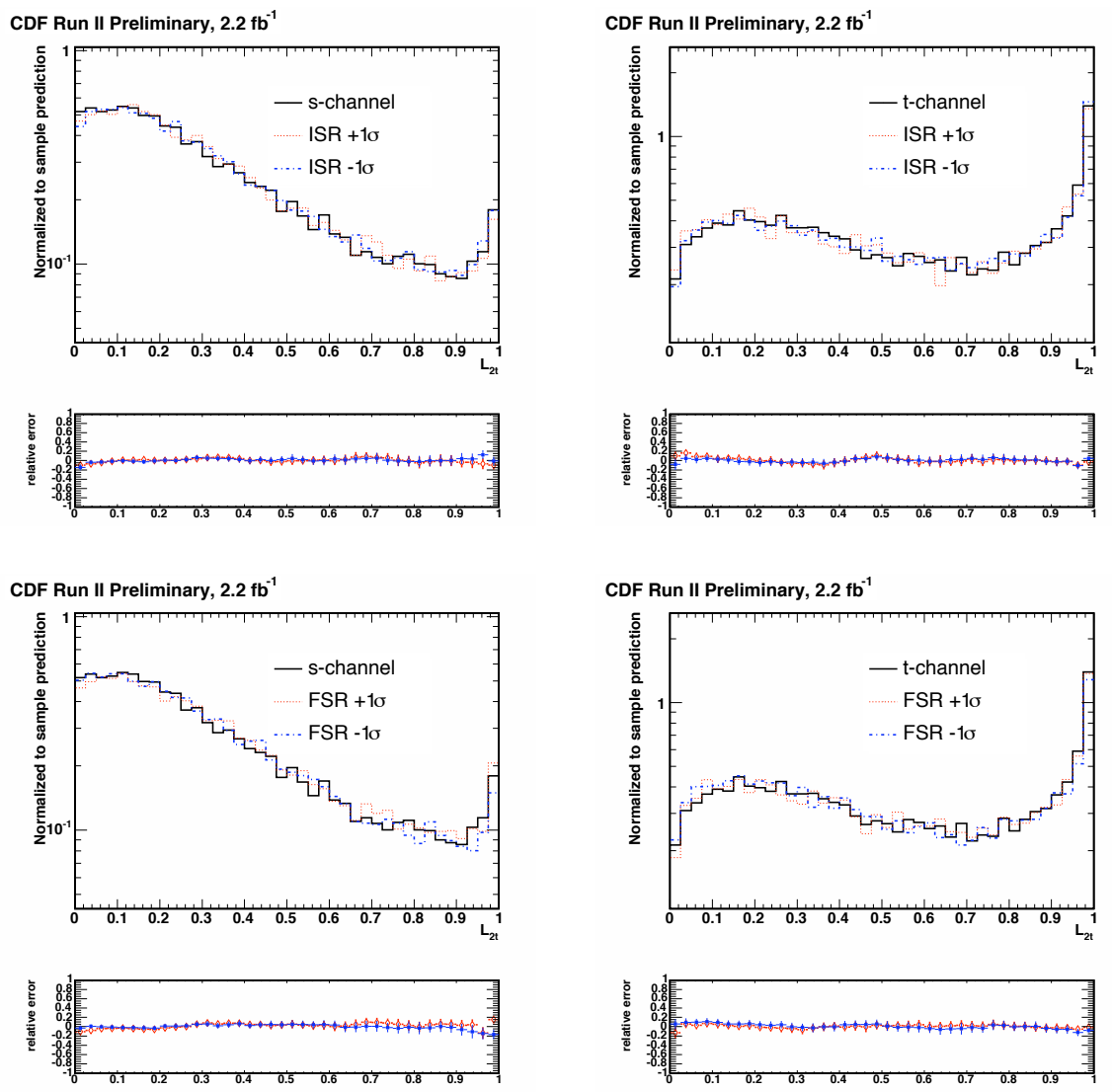

Figure B.16: FSR and ISR shape systematics for the $s$-channel and $t$-channel signal samples in the three-jet bin. 

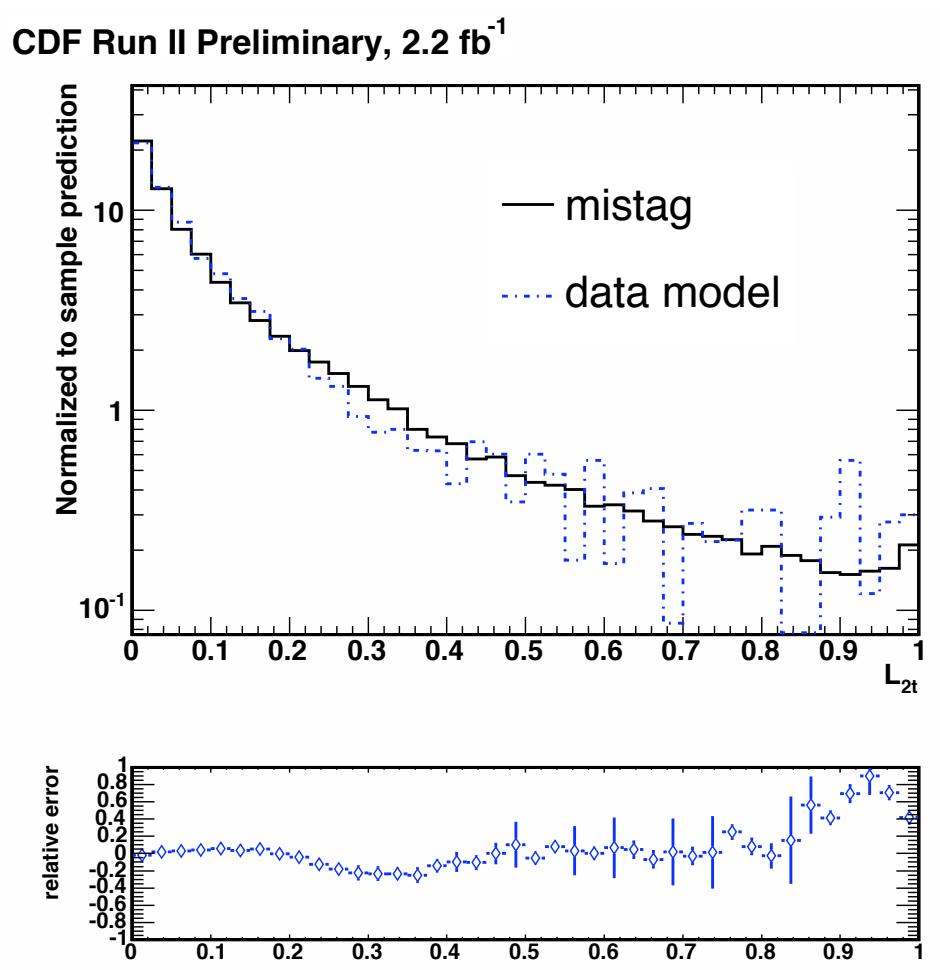

Figure B.17: Comparison between mistag model and pretag data weighted using the mistag matrix in the 3 -jet bin. 


\section{CDF Run II Preliminary, $2.2 \mathrm{fb}^{-1}$}
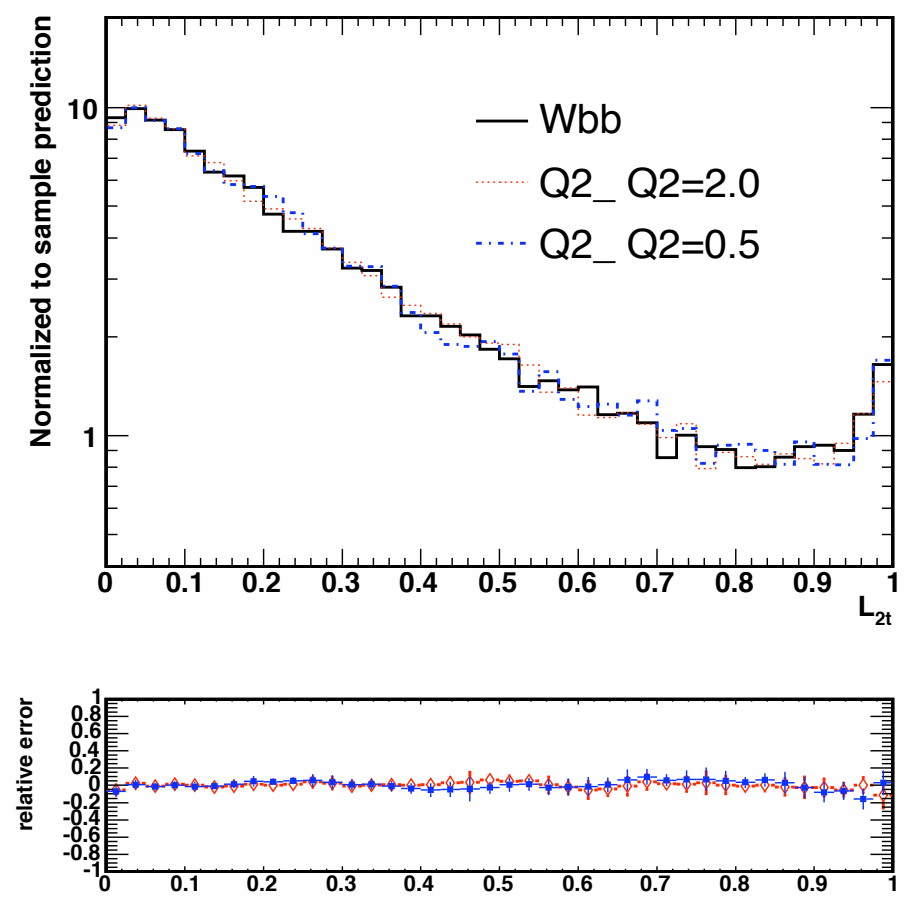

Figure B.18: $Q^{2}$ Shape systematics for the Wbb sample in the 3-jet bin.
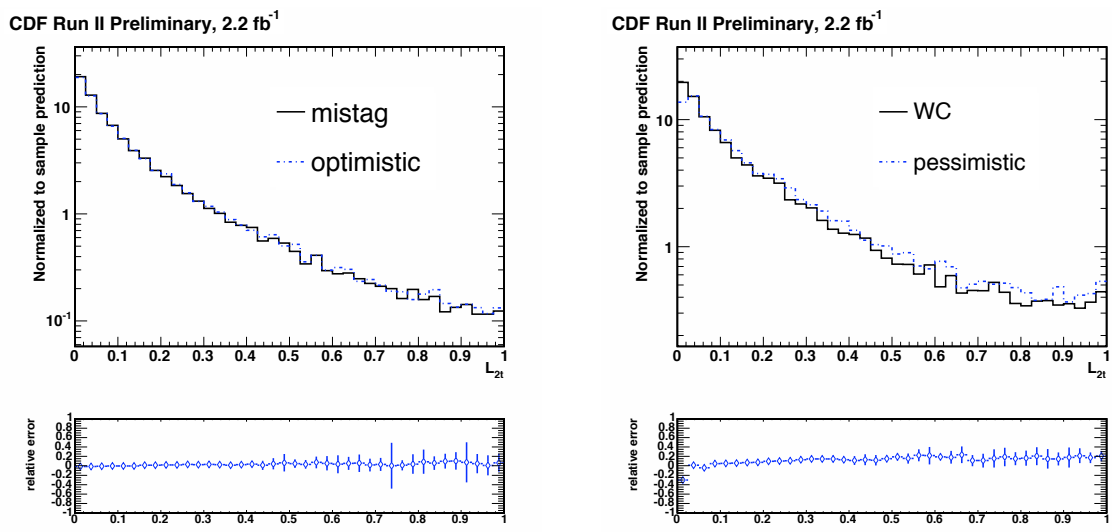

Figure B.19: NN B-Tag Shape systematics for mistag and $W c$ predictions in the 3 -jet bin. 
CDF Run II Preliminary, $2.2 \mathrm{fb}^{-1}$
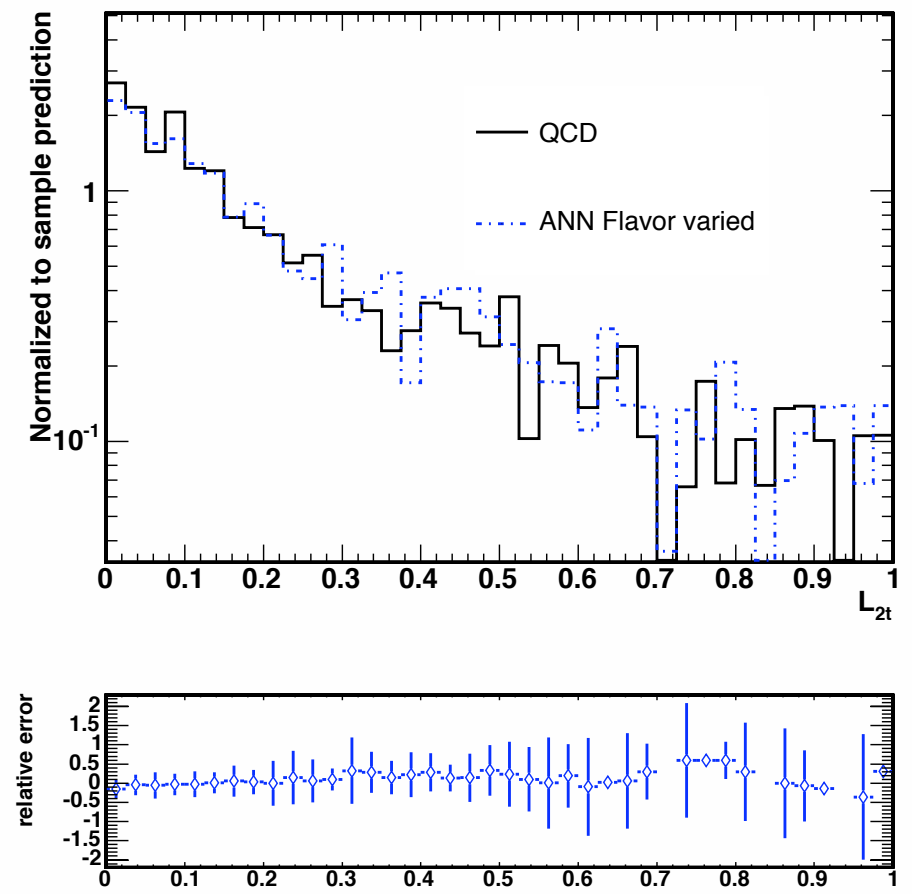

Figure B.20: Non- $W$ flavor modeling shape systematic in the 3-jet bin. Shown is the ratio of (varied-central)/central. The central value of the non-W flavor composition is estimated to be $45 \% \mathrm{~b}, 40 \%$ charm, and $15 \%$ light flavor. The systematic variation is $60 \% \mathrm{~b}, 30 \%$ charm, and $10 \%$ light flavor.
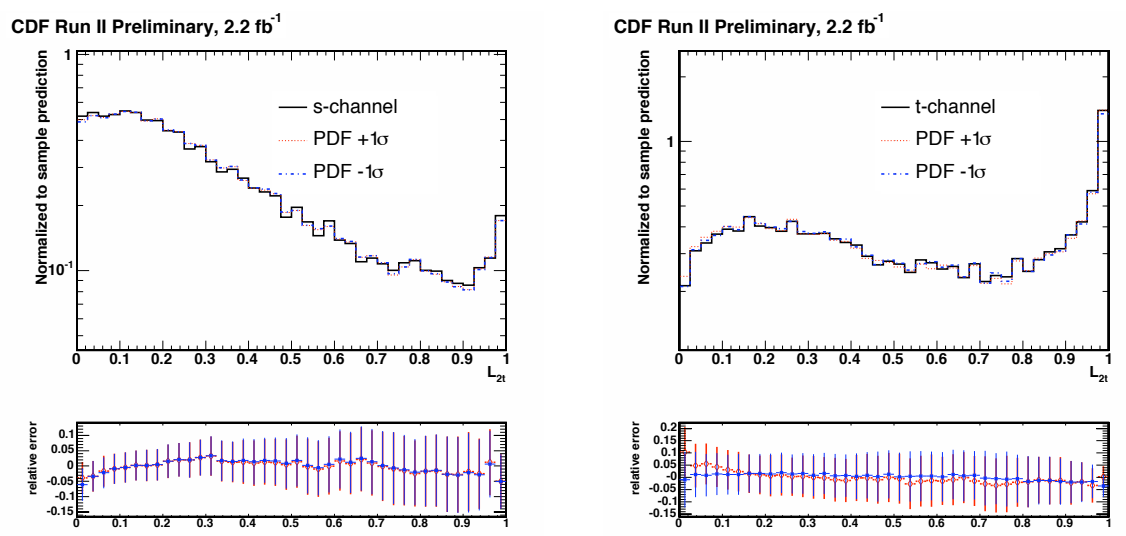

Figure B.21: PDF shape systematics for the $t$-channel and $s$-channel signal predictions in the 3 -jet bin. 

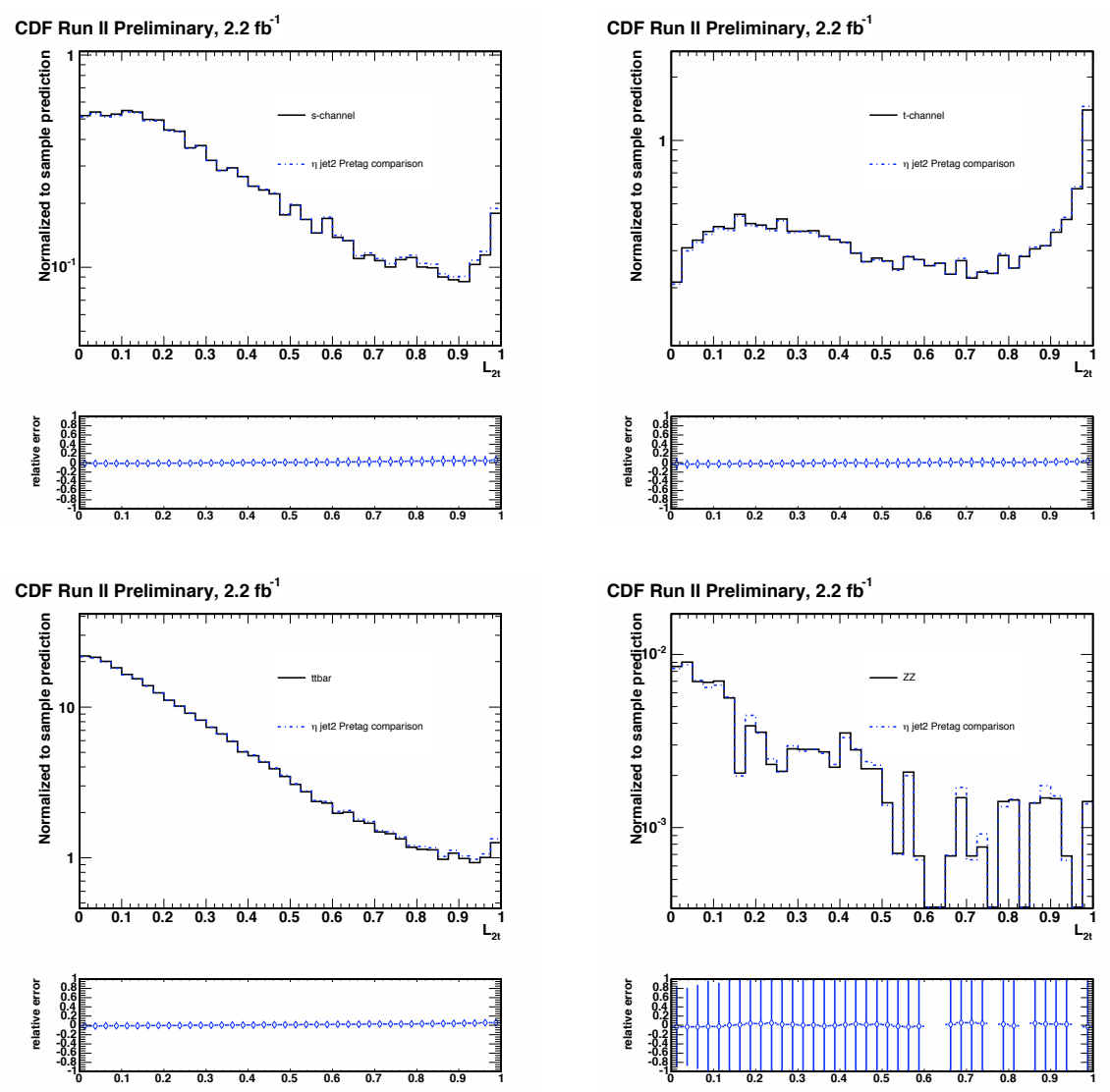

Figure B.22: Shape systematics induced by reweighting $\eta_{j 2}$ for the $s$-channel signal, the $t$-channel signal, and the $t \bar{t}$ and $Z Z$ diboson backgrounds in the 3 -jet bin. 

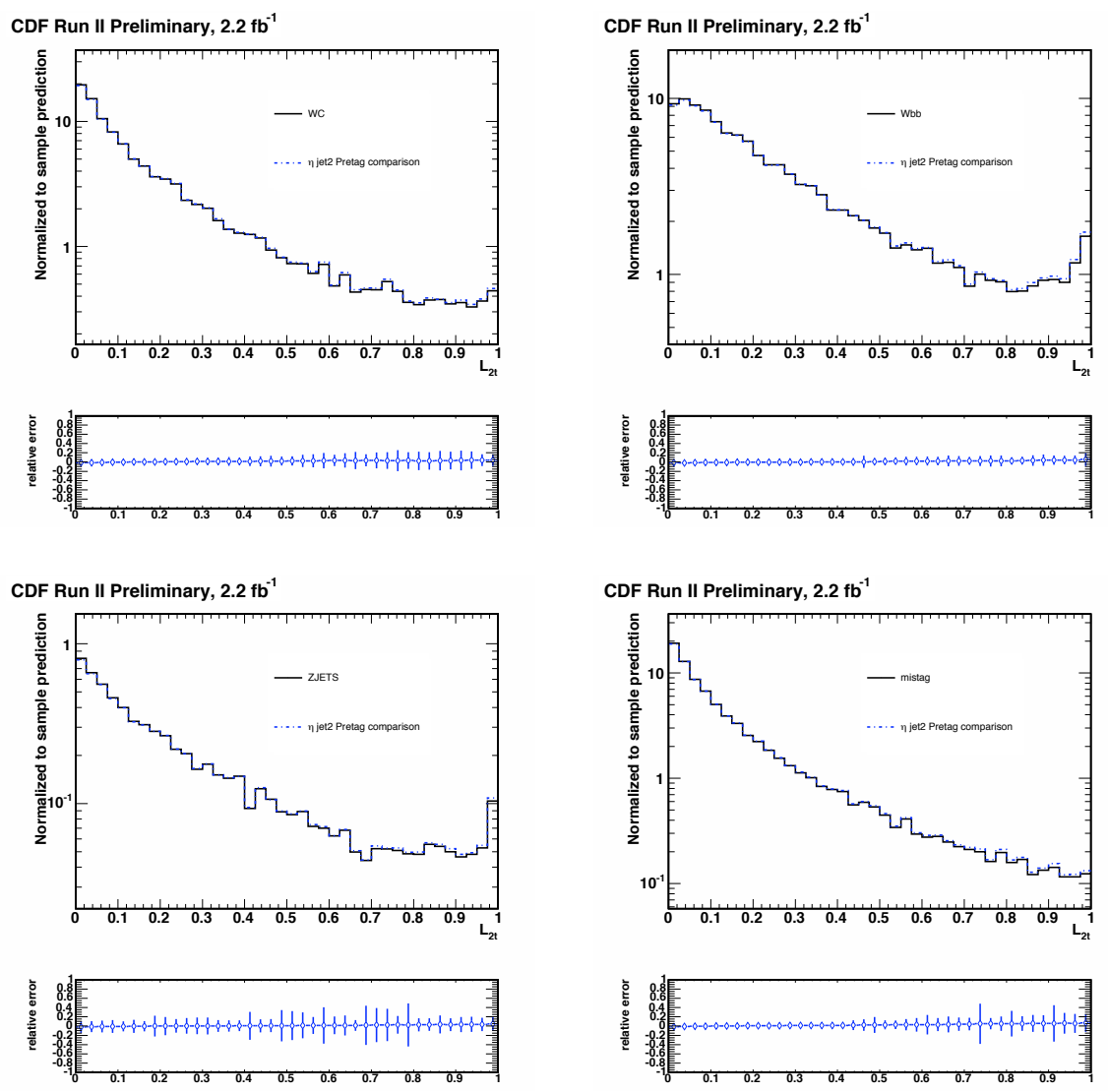

Figure B.23: Shape systematics induced by reweighting $\eta_{j 2}$ of the two jets for the $Z+$ jets, $W c / W c c, W b b$ and mistag backgrounds in the 3 -jet bin. 

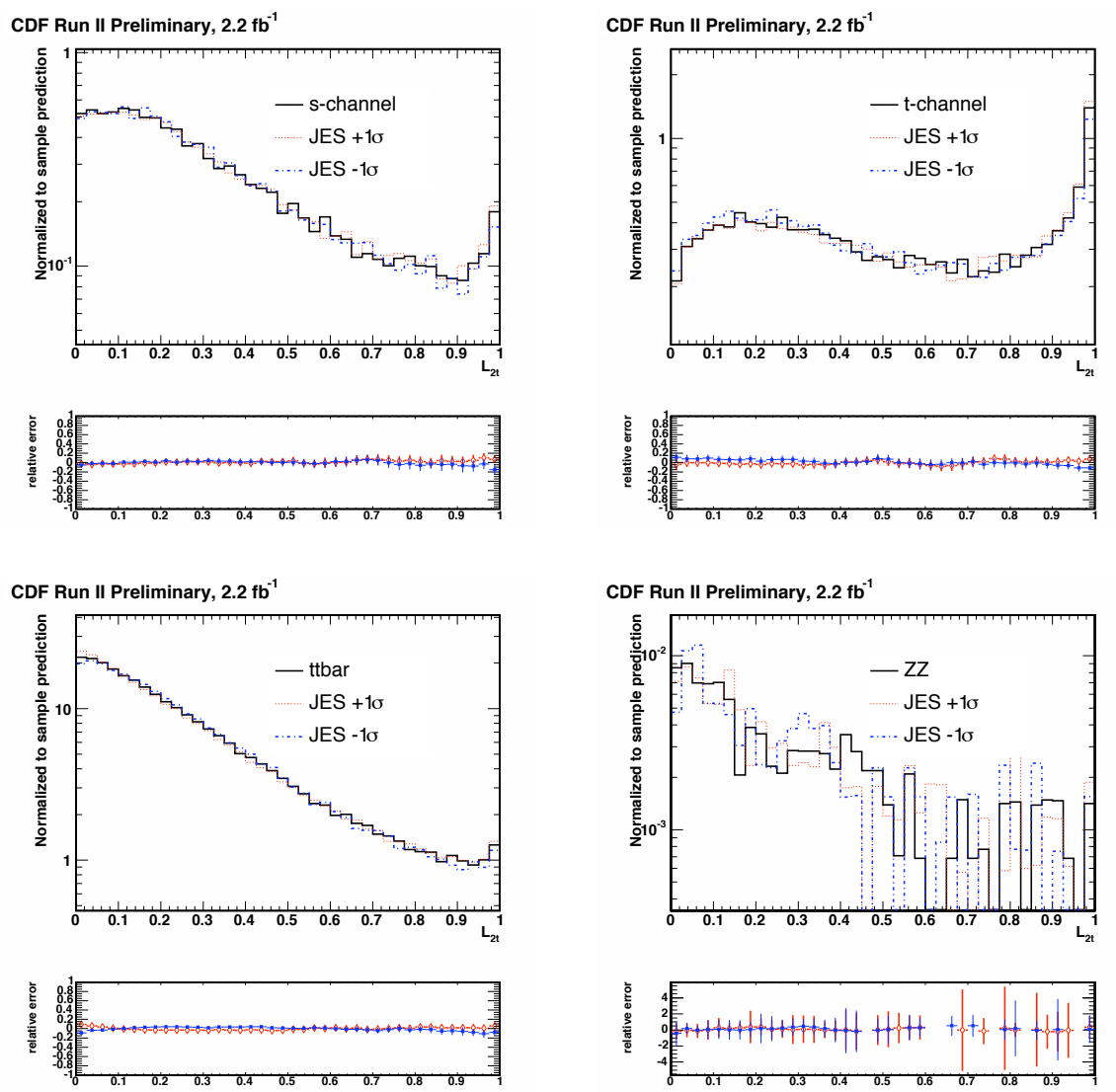

Figure B.24: Jet Energy Scale shape systematics for the $s$-channel signal, the $t$-channel signal, and the $t \bar{t}$ and $Z Z$ diboson backgrounds in the 3 -jet bin. 

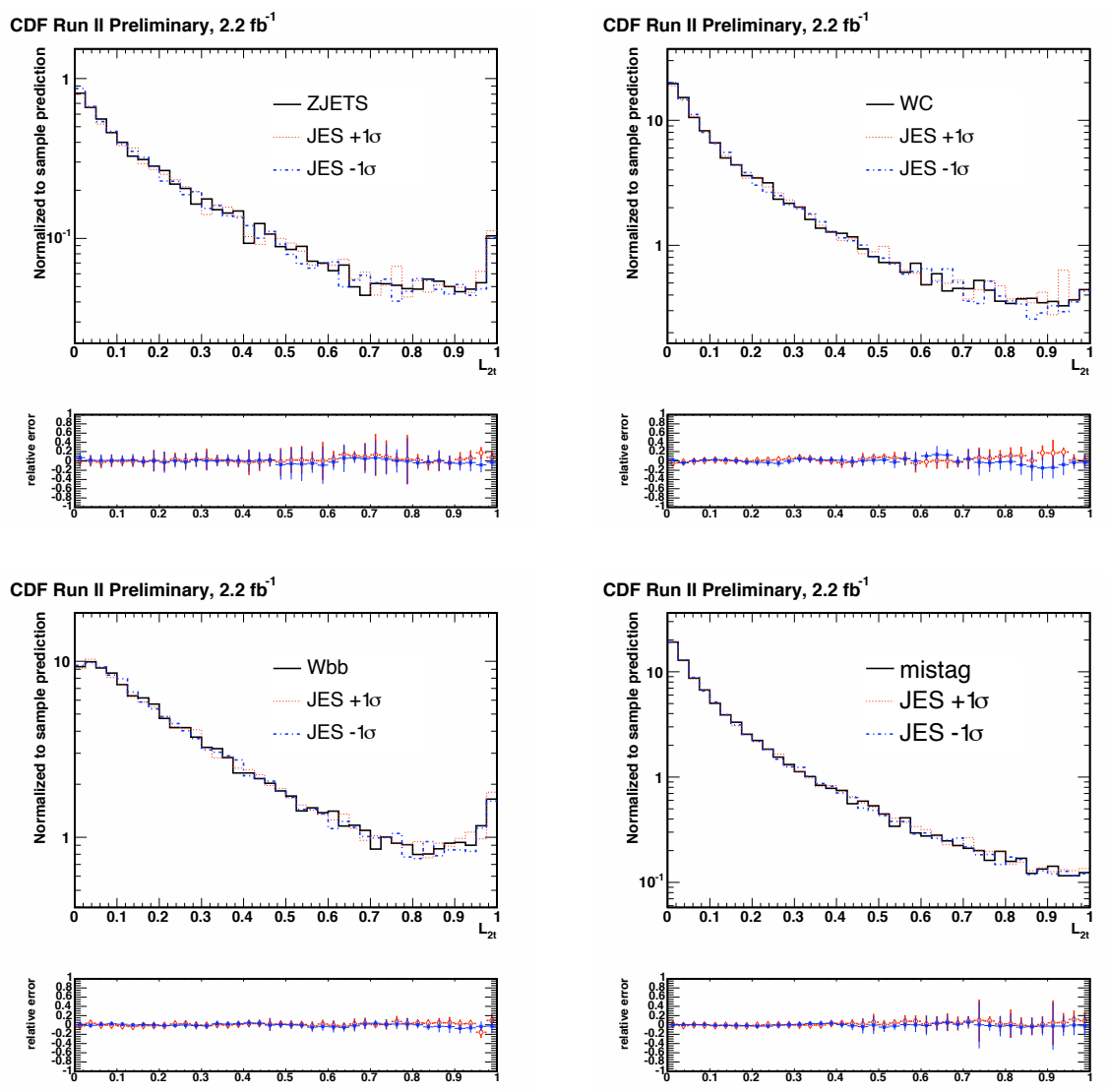

Figure B.25: Jet Energy Scale shape systematics for $Z+$ jets, $W c / W c c, W b b$ and mistag backgrounds in the 3 -jet bin. 

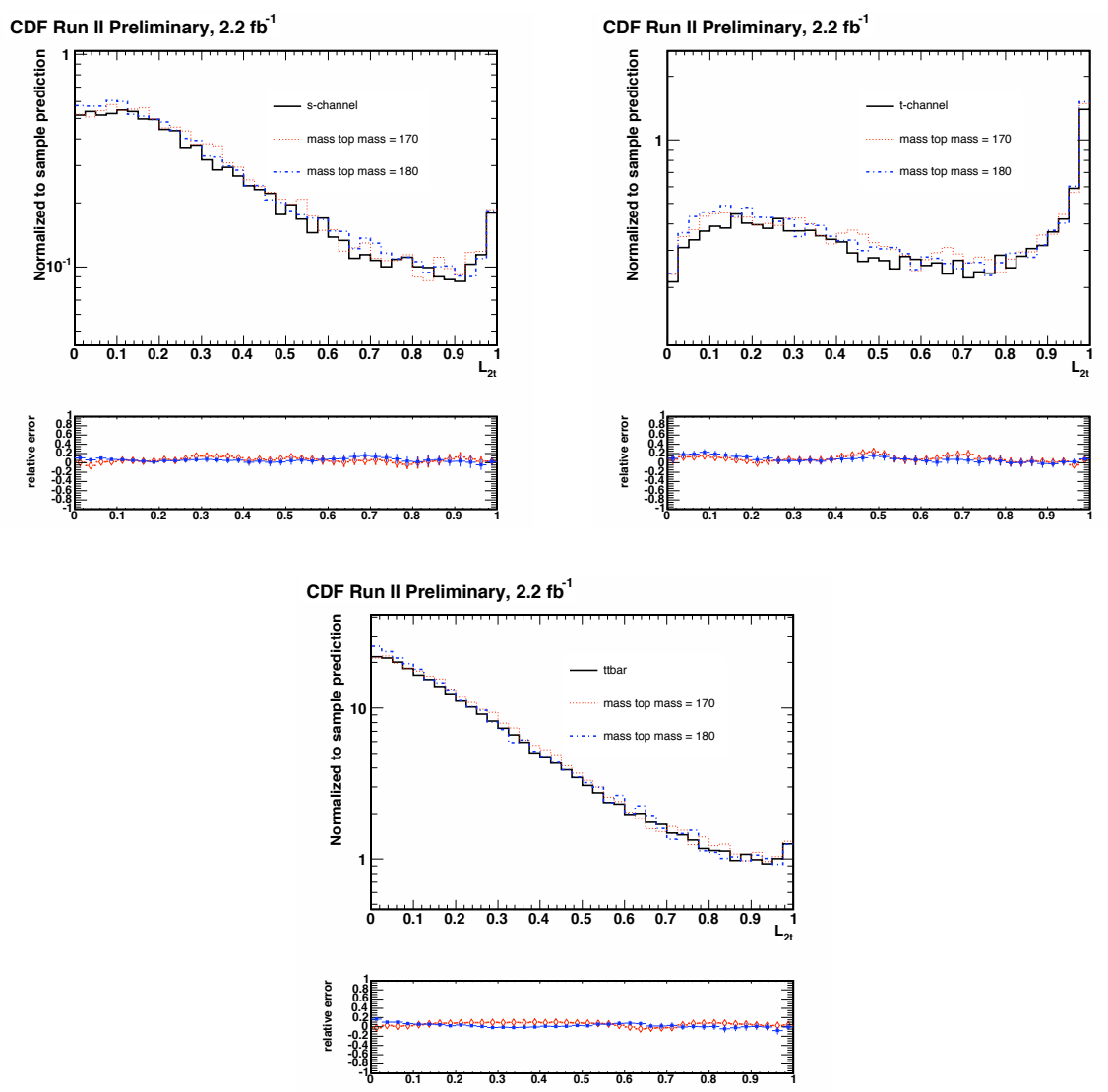

Figure B.26: Top mass shape systematics for $t$-channel, and $s$-channel signal samples and $t \bar{t}$ background in the 3 -jet bin. 


\section{Muon Chamber Monitoring and Maintenance}

In order to ensure quality analysis data, it is important that all CDF detector components run with the best performance possible. This requires a constant monitoring of the output data for possible discrepancies, as well as maintenance of detector components to fix problems as they occur. This monitoring and maintenance is the joint responsibility of many CDF scientists, often responsible for a particular detector component. The author of this thesis was part of the group responsible for the maintenance and repair of the central muon detectors.

The central muon detectors consist of the Central Muon Chambers (CMU) and the Central Muon Upgrade (CMP). The CMU consists of 2304 single-wire drift cells, divided into $4815^{\circ}$ wedges, and split into the east and west sides. The front end electronics consist of a 16-channel preamplifier board, which provides signals to a amplifier-shaper-discriminator circuit.

The CMP consists of 1064 single-wire drift cells, organized into 266 fourlayer stacks. The CMP front end electronics consist of a pair of front-end boards mounted on the chamber face. The first board distributes the high voltage to the chamber anodes and feeds the chamber signals while the second contains the preamplifier circuit.

Data from these chambers is constantly monitored for data quality by both the shift crew in the control room and by muon experts. When problems are detected in these monitoring plots, various steps can be taken to repair the muon chambers. Fortunately, the central muon chambers are relatively easy to access because of their location on the outside of the CDF detectors, so experts can go into the collision hall and repair individual muon chambers when necessary. Some examples of these monitoring plots are shown below, both for typical data taking, and for data indicating the need for central muon chamber maintenance. Typical repair options are also discussed.

A plot of the muon chamber occupancy during typical data taking is shown in Figure C.1. In this plot, the expected occupancy is given in the square points with green error bars, and the data is given by the black line. The occupancy is plotted as a function of individual stacks in the muon chambers. When the data falls above or below the predicted line, this is a possible indication of a muon chamber hardware or data problem. In general, when an individual stack is much higher or lower than the neighboring stacks, this indicates that something is unusual with that stack. Several known features are exhibited in this plot. For instance, the dip in sections 15-20 in the CMX East and West 
plots corresponds to the partially instrumented region of the detector known as the miniskirt. Similarly, CMX wedges 5 and 6 and CMP top stack 70 are all un-instrumented.

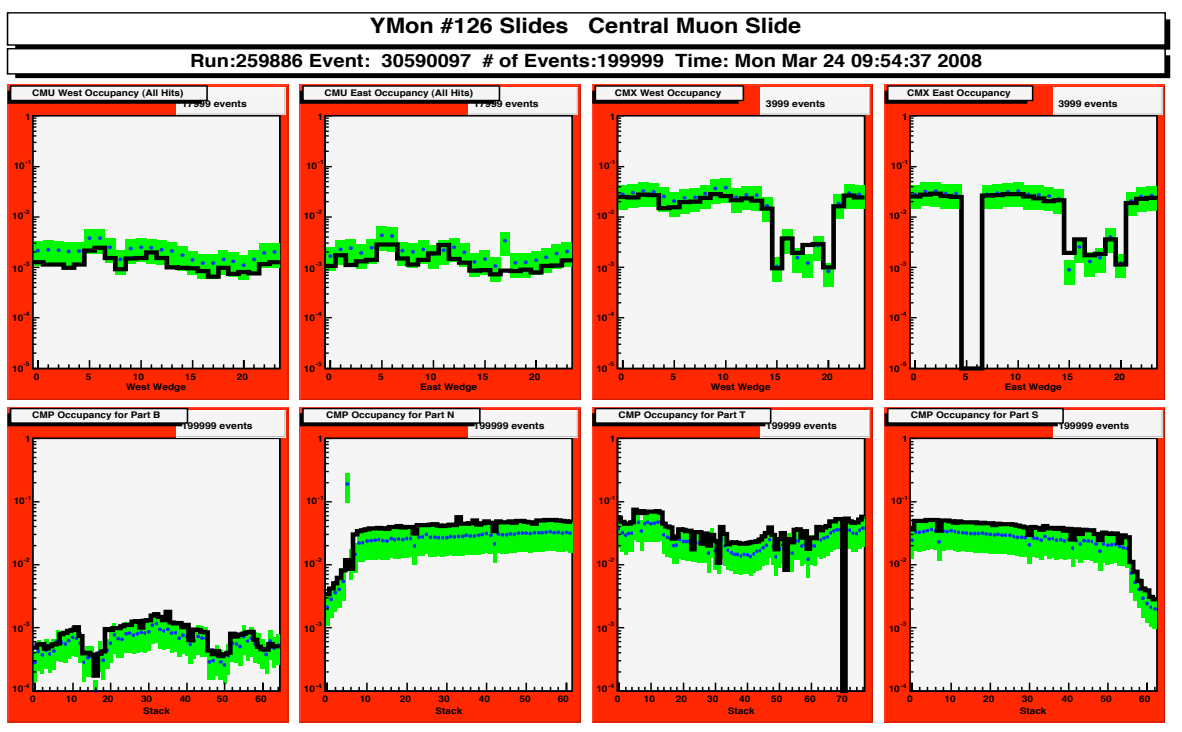

Figure C.1: Occupancy for the CMU/CMP and CMX Muon Chambers during normal data taking. The plot is divided into eight sections, CMU West, CMU East, CMX West, CMX East, CMP Bottom, CMP North, CMP Top and CMP South.

Figure C.2 shows a plot of the muon chamber occupancy in which several stacks in the CMP have higher occupancies than normal, known as 'noisy' stacks. This is often caused by oscillations in the chamber preamplifier cards. Sometimes noisy stacks can be fixed by simply power cycling the stack or by turning off the low voltage to the stack for a period of time. Other times an access is needed in which case the stack can be repaired by either replacing the preamplifier card with an updated card designed to prevent oscillations, or by putting copper tape on the chamber, which provides better grounding between the front panel and chamber cover. In the case of Figure C.2 below, the chamber was repaired by applying copper tape to the effected chambers.

Another typical problem is for one or more stacks in the muon chamber to exhibit zero occupancy. This can be due to a variety of problems with detector and trigger components. In the case of Figure C.3, shown below, one of the cables connected to the chamber had come loose during an access, and so the fix was simply to plug the cable back in tightly.

Occasionally, the CMU or CMP chambers will 'trip' or suddenly loose high voltage. The occasional trip can be quickly remedied by simply turning the high voltage to the system back on. In the case of several trips occurring in a very short time, more action is sometimes necessary. In some cases the issue can be resolved by turning the chamber off and waiting for a few hours. Trips 


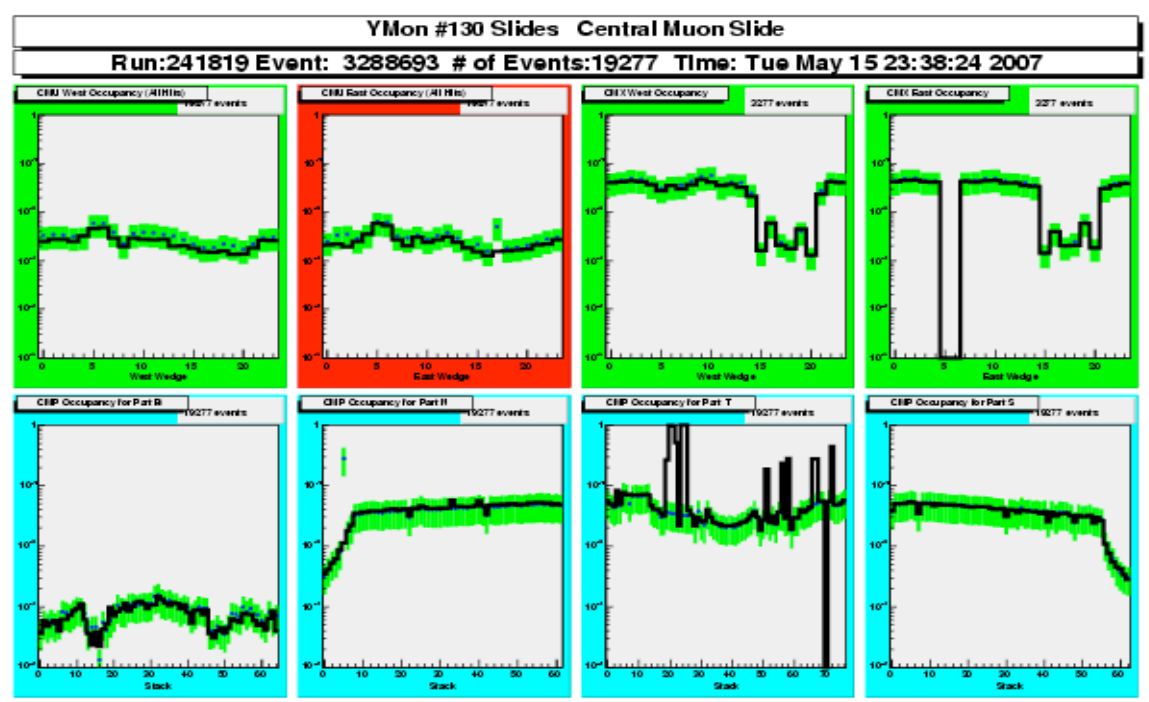

Figure C.2: Occupancy for the CMU/CMP and CMX Muon Chambers, exhibiting several hot CMP channels. The plot is divided into eight sections, CMU West, CMU East, CMX West, CMX East, CMP Bottom, CMP North, CMP Top and CMP South.

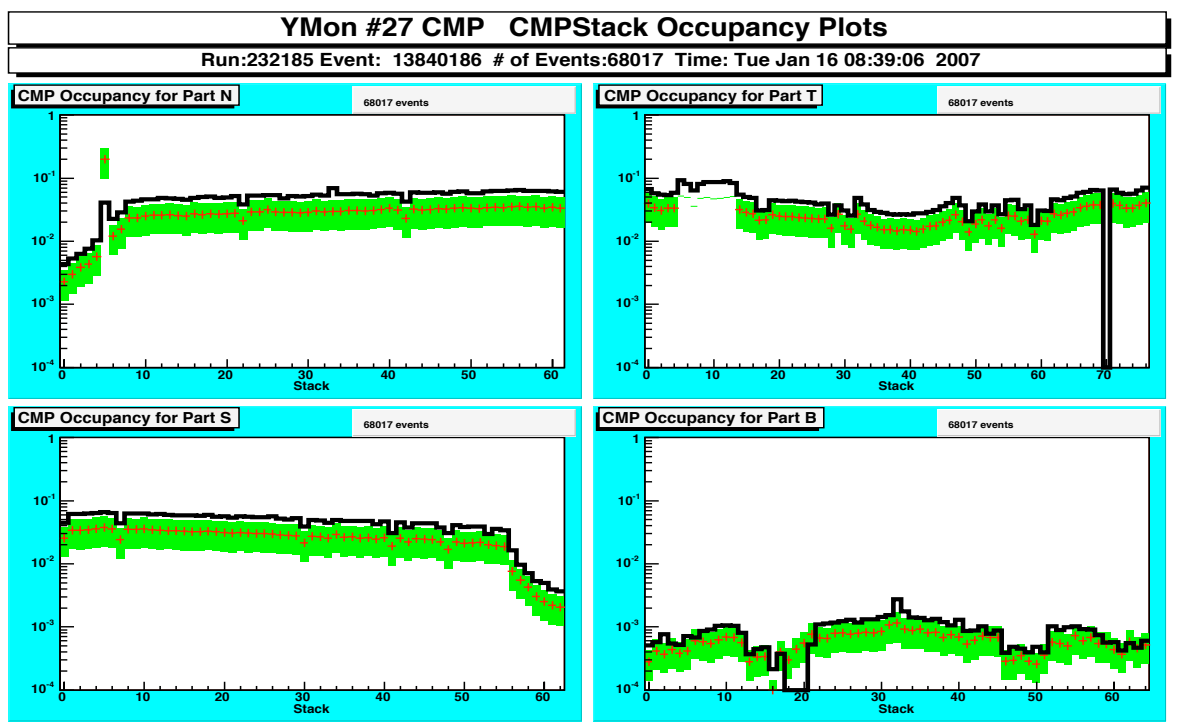

Figure C.3: Occupancy for the CMU/CMP and CMX Muon Chambers, exhibiting a dead CMP channels. The plot is divided into eight sections, CMU West, CMU East, CMX West, CMX East, CMP Bottom, CMP North, CMP Top and CMP South. 
are more frequent when the humidity is high, so if this is found to be the case it is often beneficial to wait until the detector becomes less humid and then turn high voltage to the affected muon chamber back on. In some cases, a given CMU or CMP chamber may be malfunctioning and causing many trips. In this case, it is necessary to try and isolate the exact broken chamber by manually powering each chamber in effected sections until the chamber which trips is found. Sometimes the parameters can be changed in the high voltage software to make a particular muon chamber slightly less sensitive to high voltage spikes and less prone to tripping.

\section{C.1 CMP Aging}

Another component of maintaining the CMP chambers is to monitor the behavior of the chambers over time, to check for problems associated with aging chambers. For this check, the hit width from the CMP chamber is monitored. The CMP hit width is defined as the time-over-threshold of the pulse from the anode, which is roughly proportional to the pulse height and is sensitive to the gain in the chambers. For this purpose, an offline event selection was applied to select $\mathrm{J} / P$ si- $>\mu \mu$ events, as these events constitute a high statistics sample of real muons. During this offline processing, further cuts were made requiring two CMP muons with invariant mass consistent with the $\mathrm{J} /$ Psi mass peak. Plots of the hit width were were also made for the upper and lower half of the detectors, as the chambers located at the top of the detector and on the upper walls draw significantly more current than chambers on the lower walls and underneath the detector. Plots are shown for all chambers (Figure C.4), and the upper (Figure C.5) and lower chambers (Figure C.6). These plots show that no significant aging effects were observed for the time period from August 2005-June 2008. 


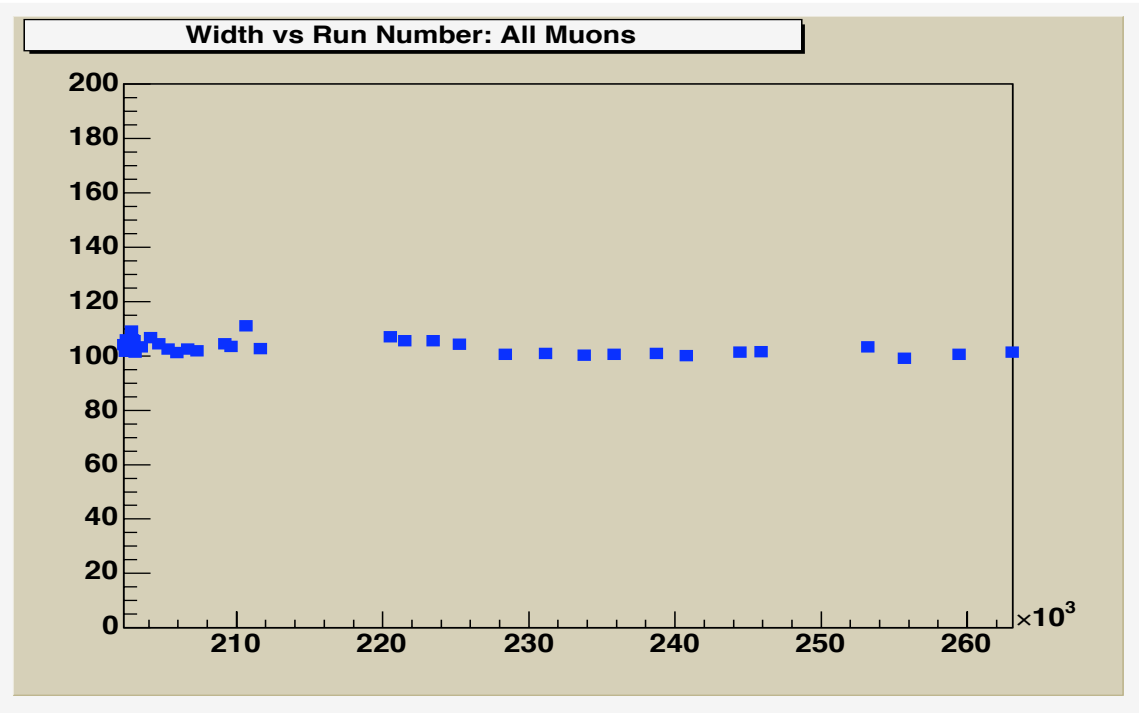

Figure C.4: $\mathrm{J} / \Psi$ hit width for CMP chambers.

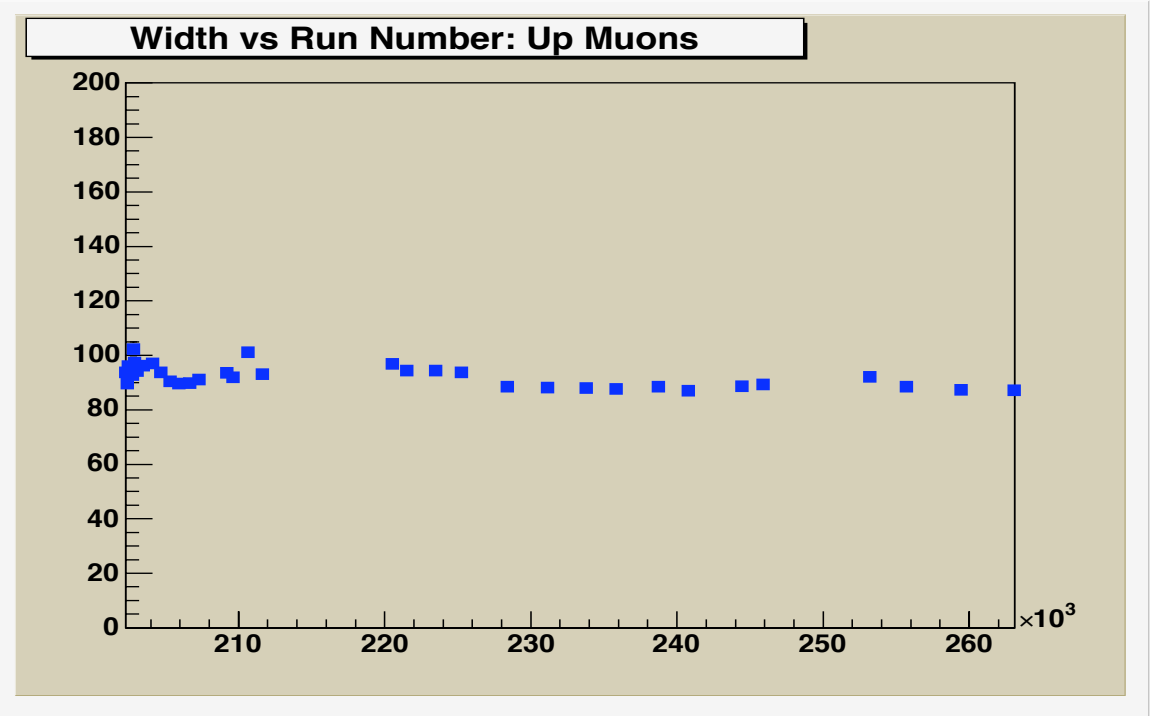

Figure C.5: J/ $/$ hit width for upper CMP chambers. 


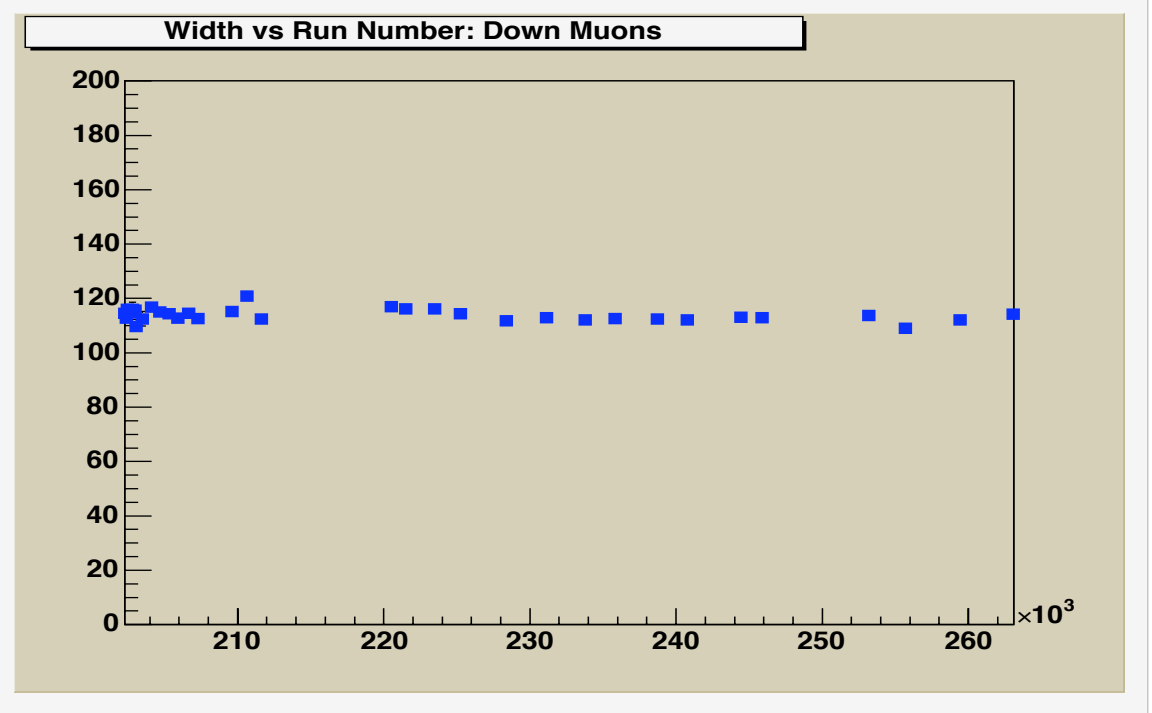

Figure C.6: J/ $\Psi$ hit width for lower CMP chambers. 


\section{$D$ The CKM Matrix and the Higgs Field}

A short discussion of the relationship between the CKM matrix and the Higgs field was presented in Chapter 3.1. In that chapter, we briefly alluded to the fact that the CKM matrix can be derived from the Yukawa term of the Standard Model Lagrangian, however no derivation or discussion was presented. The purpose of this appendix is to provide such a discussion and to further explore some of the symmetries of the Yukawa term in the Standard Model Lagrangian.

The Yukawa term in the Standard Model Lagrangian couples the left handed quark doublets, $Q_{L}$ with the right handed quark singlets $\left(d_{R}\right.$ and $\left.u_{R}\right)$, as shown in Equation D.1.

$$
\mathcal{L}_{Y}=-Y_{i j}^{d} \bar{Q}_{L i} \phi d_{R j}-Y_{i j}^{u} \bar{Q}_{L i} \epsilon \phi^{*} u_{R j}-Y_{j i}^{d *} \bar{d}_{R j} \phi^{\dagger} Q_{L i}+Y_{j i}^{u *} \bar{u}_{R j} \phi^{T} \epsilon Q_{L i}
$$

Here, $Y$ is a $3 \times 3$ complex matrix, $\mathrm{i}, \mathrm{j}$ indicate quark generations and $\epsilon$ is the $2 \times 2$ antisymmetric tensor. $\phi$ is the Standard Model Higgs field, a two-component object with complex entries. It can be parametrized as:

$$
\phi(x)=U(x)(1 / \sqrt{2})\left(\begin{array}{c}
0 \\
v+h(x)
\end{array}\right)
$$

Here $h(x)$ is a real valued field with $\langle h(x)>=0$ and $U(x)$ is a general $\mathrm{SU}(2)$ gauge transformation. The coupling of the Higgs field to the quarks does not follow from a gauge principle and therefore need not have restrictions on the coupling that prevent mixing. Thus, the theory is given by the most general renormalizable gauge-invariant couplings, including the generation mixing $Y^{u}$ and $Y^{d}$ terms.

Note here that the Yukawa Lagrangian terms are invariant under SU(2). This fact can be demonstrated by examining the first two terms in the Lagrangian (it then follows that the hermitian conjugate terms are also invariant under the rotation). Under an $\mathrm{SU}(2)$ rotation, $Q_{L} \rightarrow U Q_{L}, \phi \rightarrow U \phi$ and $d_{R}$ is

unchanged. Here, $U$ is a member of the $\mathrm{SU}(2)$ group. The first Yukawa term in the Standard Model Lagrangian then transforms as following:

$$
\begin{array}{r}
Y_{i j}^{d} \bar{Q}_{L i} \phi d_{R j}^{I} \rightarrow Y_{i j}^{d} Q_{L i}^{I \dagger} U^{\dagger} \gamma^{o} U \phi d_{R j}^{I} \\
=Y_{i j}^{d} Q_{L i}^{I \dagger} U^{\dagger} U \gamma^{o} \phi d_{R j}^{I} \\
=Y_{i j}^{d} Q_{L i}^{I \dagger} \gamma^{o} \phi d_{R j}^{I} \\
=Y_{i j}^{d} \bar{Q}_{L i} \phi d_{R j}^{I}
\end{array}
$$


Here, we have made use of the unitary property of $\mathrm{U}$, namely that $U^{\dagger} U=I$. Therefore, the first term in the Yukawa portion of the Lagrangian is invariant under $\mathrm{SU}(2)$ rotation due to the unitary property of such a rotation.

Similarly, the second term of the Yukawa Lagrangian transforms as following under an $\mathrm{SU}(2)$ rotation:

$$
Y_{i j}^{u} \bar{Q}_{L i} \epsilon \phi^{*} u_{R j} \rightarrow Y_{i j}^{u} \bar{Q}_{L i} U^{\dagger} \epsilon U^{*} \phi^{*} u_{R j}
$$

$U^{\dagger} \epsilon U^{*}=\left(U^{T} \epsilon U\right)^{*}=\epsilon$, because $U^{T} \epsilon U=|U| \epsilon=\epsilon$. Here, we have invoked the 'special' property of the $\mathrm{SU}(2)$ group, that $|U|=1$. Thus, equation D.7 becomes:

$$
\begin{array}{r}
Y_{i j}^{u} \bar{Q}_{L i} \epsilon \phi^{*} u_{R j} \rightarrow Y_{i j}^{u} \bar{Q}_{L i} U^{\dagger} \epsilon U^{*} \phi^{*} u_{R j} \\
=Y_{i j}^{u} \bar{Q}_{L i} \epsilon \phi^{*} u_{R j}
\end{array}
$$

Thus, the second term of the Yukawa Lagrangian is invariant under SU(2) rotation due to the 'special' property of the rotation.

This Yukawa portion of the Lagrangian can similarly be shown to be invariant under weak hyercharge, $U(1)_{Y}$. The Lagrangian in fact must be invariant under $U(1)_{Y}$, since this is a fundamental symmetry of the theory, broken by the vacuum expectation value but not in the Lagrangian. This symmetry fixes the weak hypercharge quantum numbers of the right-chirality quarks. Because of the $U(1)$ symmetry, the quantum numbers of each term must sum to zero. So, for instance if we take the value of the weak hypercharge, $Y$, for $Q_{L}$ to be $1 / 6$ and the value of $Y$ for $\phi$ to be $1 / 2$, the value of $Y$ for $d_{R}$ must be $-1 / 3$ (since $-1 / 6+1 / 2-1 / 3=0)$. Similarly, the value of $Y$ for $u_{R}=2 / 3$ (since $\mathrm{Y}\left(Q_{D}\right)=1 / 6$, $\mathrm{Y}\left(u_{R}^{i}\right)=1 / 2$ and $\left.-1 / 6-1 / 2+2 / 3=0\right)$.

Having examined the symmetries of the Yukawa Lagrangian, we can now proceed with our derivation of the origin of the CKM matrix. The next step is to choose the unitary gauge in which only the lower component of $\phi$ is nonzero and that component is real, $\langle\phi\rangle=(0, v / \sqrt{2})$. With this choice, $\bar{Q}_{L} \phi \rightarrow$ $1 / \sqrt{2}(h+\nu) \bar{d}_{L}$ and and $\bar{Q}_{L} \epsilon \phi^{*} \rightarrow 1 / \sqrt{2}(h+\nu) \bar{u}_{L}$. Then the Yukawa Lagrangian becomes:

$$
\mathcal{L}_{Y}=-Y_{i j}^{u} \frac{h+\nu}{\sqrt{2}} \bar{d}_{L}^{i} d_{R}^{j}-Y_{i j}^{d} \frac{h+\nu}{\sqrt{2}} \bar{u}_{L}^{i} u_{k}^{j}+h . c
$$

Using singular value decomposition, any matrix, $M$ can be written in the form $M=U E V^{\dagger}$ where $U, V$ are unitary matrices and $E$ is a non-negative diagonal matrix. It follows that $E=U^{\dagger} M V$. Thus, we can define unitary matrices $V_{L}^{u}, V_{L}^{d}, V_{R}^{u}$ and $V_{R}^{d}$ such that $Y_{\text {diag }}^{d}=V_{L}^{d \dagger} Y^{d} V_{R}^{d}$ and $Y_{\text {diag }}^{u}=V_{L}^{u \dagger} Y^{u} V_{L}^{d}$. Now, define $d_{L}^{\prime}$ and $d_{R}^{\prime}$ such that $d_{L}=V_{L}^{d} d_{L}^{\prime}$ and $d_{R}=V_{R}^{d} d_{R}^{\prime}$, and Equation D.10 becomes: 


$$
\begin{array}{r}
\mathcal{L}_{Y}=-\frac{h+\nu}{\sqrt{2}} \bar{d}_{L}^{\prime} V_{L}^{d \dagger} Y^{d} V_{R}^{d} d_{R}^{\prime}-\frac{h+\nu}{\sqrt{2}} \bar{u}_{L}^{\prime} V_{L}^{u \dagger} Y_{u} V_{R}^{u} u_{R}^{\prime}+h . c \\
=-\frac{h+\nu}{\sqrt{2}} \bar{d}_{L}^{\prime} Y_{\text {diag }}^{u} d_{R}^{\prime}-\frac{h+\nu}{\sqrt{2}} \bar{u}_{L}^{\prime} Y_{\text {diag }}^{d} u_{R}^{\prime}+h . c
\end{array}
$$

Now, let $m_{u}^{i}=\frac{v}{\sqrt{2}} Y_{\text {diag }}^{u} v$ and $m_{d}^{i}=\frac{v}{\sqrt{2}} Y_{\text {diag }}^{d} v$. Then equation D.12 becomes

$$
\mathcal{L}=-(1+h / \nu) m_{d}^{i} \bar{d}_{L}^{i} d_{R}^{i}-(1+h / \nu) m_{u}^{i} \bar{u}_{L}^{i} u_{R}^{i}+h . c .
$$

To understand how mixing is generated by these four unitary matrices, it is useful to see the effect of the substitution of $d=V^{d} d^{\prime}$ and $u=V^{u} u^{\prime}$ on the neutral and charged currents. For the neutral EM current, the portion of $J_{E M}^{\mu}$ involved the quark terms can be written as following

$$
\begin{array}{r}
J_{E M}^{\mu}=\bar{u} \gamma^{\mu}(+2 / 3) u+\bar{d} \gamma^{\mu}(-1 / 3) d \\
=\bar{u}^{\prime} U^{u \dagger} \gamma^{\mu}(+2 / 3) u^{\prime} U^{u}+\bar{d}^{\prime} U^{d \dagger} \gamma^{\mu}(-1 / 3) U^{d} d^{\prime} \\
=\bar{u}^{\prime} \gamma^{\mu}(+2 / 3) u^{\prime}+\bar{d}^{\prime} \gamma^{\mu}(-1 / 3) d^{\prime}
\end{array}
$$

The neutral $\mathrm{Z}$ current similarly exhibits no mixing terms, and can be written as follows:

$$
\begin{aligned}
& J_{Z}^{\mu}=\bar{u}_{L} \gamma^{\mu}\left(1 / 2-2 / 3 \sin ^{2} \theta_{W}\right) u_{L}+\bar{u}_{R} \gamma^{\mu}\left(-2 / 3 \sin ^{2} \theta_{w}\right) u_{R} \\
& +\bar{d}_{L} \gamma^{\mu}\left(-1 / 2+1 / 3 \sin ^{2} \theta_{w}\right) d_{L}+\bar{d}_{R} \gamma^{\mu}\left(1 / 3 \sin ^{2} \theta_{w}\right) d_{R} \\
& =\bar{u}_{L}^{\prime} U_{L}^{u \dagger} \gamma^{\mu}\left(1 / 2-2 / 3 \sin ^{2} \theta_{W}\right) U_{L}^{u} u_{L}^{\prime}+\bar{u}_{R} U_{R}^{u \dagger} \gamma^{\mu}\left(-2 / 3 \sin ^{2} \theta_{w}\right) U_{R}^{u} u_{R}^{\prime} \\
& +\bar{d}_{L}^{\prime} U_{L}^{d \dagger} \gamma^{\mu}\left(-1 / 2+1 / 3 \sin ^{2} \theta_{w}\right) U_{L}^{d} d_{L}^{\prime}+\bar{d}^{\prime}{ }_{R} U_{R}^{d \dagger} \gamma^{\mu}\left(1 / 3 \sin ^{2} \theta_{w}\right) U_{R}^{d} d_{R}^{\prime} \\
& =\bar{u}_{L}{ }_{L} \gamma^{\mu}\left(1 / 2-2 / 3 \sin ^{2} \theta_{W}\right) u_{L}^{\prime}+\bar{u}_{R}{ }_{R} \gamma^{\mu}\left(-2 / 3 \sin ^{2} \theta_{w}\right) u_{R}^{\prime} \\
& +\bar{d}^{\prime}{ }_{L} \gamma^{\mu}\left(-1 / 2+1 / 3 \sin ^{2} \theta_{w}\right) d_{L}^{\prime}+\bar{d}_{R}^{\prime} \gamma^{\mu}\left(1 / 3 \sin ^{2} \theta_{w}\right) d_{R}^{\prime}
\end{aligned}
$$

In the current that couples to the $\mathrm{W}$ boson field, however, the $U_{u}$ and $U_{d}$ terms do not cancel out. For the $W^{+}$boson:

$$
\begin{array}{r}
J_{W+}^{\mu}=1 / \sqrt{2}\left(\bar{u}_{L} \gamma^{\mu} d_{L}\right) \\
=1 / \sqrt{2}\left(\bar{u}^{\prime}{ }_{L} U_{L}^{u \dagger} \gamma^{\mu} U_{L}^{d} d_{L}^{\prime}\right) \\
=1 / \sqrt{2}\left(\bar{u}^{\prime}{ }_{L} \gamma^{\mu} U_{L}^{u \dagger} U_{L}^{d} d_{L}^{\prime}\right) \\
=1 / \sqrt{2}\left(\bar{u}^{\prime}{ }_{L} \gamma^{\mu} V d_{L}^{\prime}\right)
\end{array}
$$

Here $V=U_{L}^{u \dagger} U_{L}^{d}$. This indicates the $W^{ \pm}$interactions actually link the three $u_{L}^{i}$ quarks with the $d i_{L}$ triplet via a unitary rotation given by $V$. Since no such coupling is given for either of the neutral currents, there are no flavor changing neutral currents at tree level. Note that $V^{\dagger} V=\left(U_{L}^{u \dagger} U_{L}^{d}\right)^{\dagger}\left(U_{L}^{u \dagger} U_{L}^{d}\right)=$ 
$U_{L}^{d \dagger} U_{L}^{u} U_{L}^{u \dagger} U_{L}^{d}=U_{L}^{d \dagger} U_{L}^{d}=1$, so $V$ is unitary. Otherwise, the matrix $V$ can have complex elements, and the values of the elements are not explicitly given by theory. $V$ is known as the Cabibbo-Kobayashi-Maskawa (CKM) matrix. 


\section{References}

[1] CDF collaboration. Evidence for Single Top Quark Production in $1.51 \mathrm{fb}^{-1}$ of CDF II Data using the Matrix Element Technique. Public CDF note 8968, 2007. (http://www-cdf.fnal.gov/physics/new/top/2007/singletop/ME1_5fb/cdf8968_STME_pub.ps)

[2] V. M. Abazov et al. [D0 Collaboration], Phys. Rev. Lett. 98, 181802 (2007) [arXiv:hep-ex/0612052].

[3] V. M. Abazov et al. [D0 Collaboration], arXiv:0803.0739 [hep-ex].

[4] R. Blair et al. [CDF-II Collaboration], "The CDF-II detector: Technical design report," CITATION = FERMILAB-PUB-96-390-E;

[5] D. E. Acosta et al. [CDF Collaboration], Phys. Rev. D 71, 052003 (2005) [arXiv:hep-ex/0410041].

[6] C. S. Hill [On behalf of the CDF Collaboration], Nucl. Instrum. Meth. A 530, 1 (2004).

[7] A. Sill [CDF Collaboration], Nucl. Instrum. Meth. A 447, 1 (2000).

[8] A. A. Affolder et al. [CDF Collaboration], Nucl. Instrum. Meth. A 453, 84 (2000).

[9] A. A. Affolder et al. [CDF Collaboration], Nucl. Instrum. Meth. A 526, 249 (2004).

[10] D. Acosta et al. [CDF-II Collaboration], Nucl. Instrum. Meth. A 518, 605 (2004).

[11] L. Balka et al. [CDF Collaboration], Nucl. Instrum. Meth. A 267, 272 (1988).

[12] S. R. Hahn et al. [CDF Collaboration], Nucl. Instrum. Meth. A 267, 351 (1988).

[13] S. Bertolucci et al. [CDF Collaboration], Nucl. Instrum. Meth. A 267, 301 (1988).

[14] M. G. Albrow et al. [CDF Collaboration], Nucl. Instrum. Meth. A 480, 524 (2002).

[15] G. Apollinari, K. Goulianos, P. Melese and M. Lindgren, Nucl. Instrum. Meth. A 412, 515 (1998).

[16] G. Ascoli et al., Nucl. Instrum. Meth. A 268, 33 (1988).

[17] T. Dorigo [CDF Collaboration], Nucl. Instrum. Meth. A 461, 560 (2001).

[18] D. Acosta et al., Nucl. Instrum. Meth. A 494, 57 (2002). 
[19] B. Ashmanskas et al. [CDF-II Collaboration], Nucl. Instrum. Meth. A 518, 532 (2004) [arXiv:physics/0306169].

[20] E. J. Thomson et al., IEEE Trans. Nucl. Sci. 49, 1063 (2002).

[21] W. M. Yao et al. [Particle Data Group], J. Phys. G 33, 1 (2006) and 2007 partial update for the 2008 edition.

[22] [CDF Collaboration], arXiv:0803.1683 [hep-ex].

[23] F. Larios, E. Malkawi and C. P. Yuan, arXiv:hep-ph/9704288.

[24] S. L. Glashow, Nucl. Phys. 22, 579 (1961).

[25] A. Salam and J. C. Ward, Phys. Lett. 13, 168 (1964).

[26] S. Weinberg, Phys. Rev. Lett. 19, 1264 (1967).

[27] P. W. Higgs, Phys. Rev. Lett. 13, 508 (1964).

[28] M. E. Peskin and D. V. Schroeder, Reading, USA: Addison-Wesley (1995) $842 p$

[29] H. L. Lai et al. [CTEQ Collaboration], Eur. Phys. J. C 12, 375 (2000).

[30] M.R.Whalley, HEPDATA, The Durham HEP Databases (http://durpdg.dur.ac.uk/hepdata/pdf3.html)

[31] F. Abe et al. [CDF Collaboration], Phys. Rev. Lett. 74, 2626 (1995) [arXiv:hep-ex/9503002].

[32] S. Abachi et al. [D0 Collaboration], Phys. Rev. Lett. 74, 2632 (1995) [arXiv:hep-ex/9503003].

[33] CDF collaboration. Combination of CDF top quark pair production cross section mearuments with up to $760 \mathrm{pb}^{-1}$. Public CDF note 8148, 2007. (http://www-cdf.fnal.gov/physics/new/top/confNotes/cdf8148_ttbar_xs_combo.ps)

[34] V. M. Abazov et al. [D0 Collaboration], arXiv:0803.2779 [hep-ex].

[35] S. Willenbrock, arXiv:hep-ph/0211067.

[36] T. M. P. Tait, Phys. Rev. D 61, 034001 (2000) [arXiv:hep-ph/9909352].

[37] Z. Sullivan, Phys. Rev. D 70, 114012 (2004) [arXiv:hep-ph/0408049].

[38] B. W. Harris, E. Laenen, L. Phaf, Z. Sullivan and S. Weinzierl, Phys. Rev. D 66, 054024 (2002) [arXiv:hep-ph/0207055].

[39] S. S. D. Willenbrock and D. A. Dicus, Phys. Rev. D 34, 155 (1986).

[40] G. Mahlon and S. J. Parke, Phys.Rev.D 66, 010001 (2002) arXiv:hep$\mathrm{ph} / 9611367$.

[41] G. Mahlon, arXiv:hep-ph/9811219.

[42] T. Stelzer, Z. Sullivan and S. Willenbrock, Phys. Rev. D 58, 094021 (1998) [arXiv:hep-ph/9807340].

[43] D. E. Acosta et al. [CDF Collaboration], Phys. Rev. Lett. 95, 102002 (2005) [arXiv:hep-ex/0505091]. 
[44] V. M. Abazov et al. [D0 Collaboration], arXiv:0801.1326 [hep-ex].

[45] J. Alwall et al., Eur. Phys. J. C 49, 791 (2007) [arXiv:hep-ph/0607115].

[46] T. Aaltonen et al. [CDF Collaboration], Phys. Rev. Lett. 100, 161803 (2008) [arXiv:0801.3877 [hep-ex]].

[47] T. Tait and C. P. Yuan, Phys. Rev. D 63, 014018 (2001) [arXiv:hep$\mathrm{ph} / 0002798]$.

[48] T. Tait and C. P. Yuan, arXiv:hep-ph/9710372.

[49] Z. Sullivan, Phys. Rev. D 66, 075011 (2002) [arXiv:hep-ph/0207290].

[50] A. P. Heinson, A. S. Belyaev and E. E. Boos, Phys. Rev. D 56, 3114 (1997) [arXiv:hep-ph/9612424].

[51] E. Boos, L. Dudko and T. Ohl, Eur. Phys. J. C 11, 473 (1999) [arXiv:hep$\mathrm{ph} / 9903215]$.

[52] T. Han, M. Hosch, K. Whisnant, B. L. Young and X. Zhang, Phys. Rev. D 58, 073008 (1998) [arXiv:hep-ph/9806486].

[53] T. Han and J. L. Hewett, Phys. Rev. D 60, 074015 (1999) [arXiv:hep$\mathrm{ph} / 9811237]$.

[54] M. Hosch, K. Whisnant and B. L. Young, Phys. Rev. D 56, 5725 (1997) [arXiv:hep-ph/9703450].

[55] T. Tait and C. P. Yuan, Phys. Rev. D 55, 7300 (1997) [arXiv:hep$\mathrm{ph} / 9611244]$.

[56] E. Malkawi and T. Tait, Phys. Rev. D 54, 5758 (1996) [arXiv:hep$\mathrm{ph} / 9511337$.

MSSM

[57] A. Datta, P. J. O'Donnell, Z. H. Lin, X. Zhang and T. Huang, Phys. Lett. B 483, 203 (2000) [arXiv:hep-ph/0001059].

[58] S. Bar-Shalom, D. Atwood and A. Soni, Phys. Rev. D 57, 1495 (1998) [arXiv:hep-ph/9708357].

[59] G. L. Kane, G. A. Ladinsky and C. P. Yuan, Phys. Rev. D 45, 124 (1992).

[60] V. M. Abazov et al. [D0 Collaboration], arXiv:0803.3256 [hep-ex].

[61] CDF collaboration. W'-like Resonances in the $t \bar{b}$ Decay Channel with $1.9 \mathrm{fb}^{-1}$. (http://www-cdf.fnal.gov/physics/new/top/confNotes/cdf9150_- $\mathrm{W}_{t} o_{t} b_{i} n_{W}+$ Jets $\left.s_{2} . p d f^{\prime}\right)$

[62] V. M. Abazov et al. [D0 Collaboration], Phys. Rev. Lett. 99, 191802 (2007) [arXiv:hep-ex/0702005].

[63] K. Whisnant, J. M. Yang, B. L. Young and X. Zhang, Phys. Rev. D 56, 467 (1997) [arXiv:hep-ph/9702305].

[64] P. Baringer, P. Jain, D. W. McKay and L. L. Smith, Phys. Rev. D 56, 2914 (1997) [arXiv:hep-ph/9705308].

[65] E. H. Simmons, Phys. Rev. D 55, 5494 (1997) [arXiv:hep-ph/9612402]. 
[66] D. O. Carlson, E. Malkawi and C. P. Yuan, Phys. Lett. B 337, 145 (1994) [arXiv:hep-ph/9405277].

[67] T. G. Rizzo, Phys. Rev. D 53, 6218 (1996) [arXiv:hep-ph/9506351].

[68] E. Sexton-Kennedy and P. Murat, In the Proceedings of 2003 Conference for Computing in High-Energy and Nuclear Physics (CHEP 03), La Jolla, California, 24-28 Mar 2003, pp THJT001 [arXiv:physics/0306112].

[69] A. Abulencia et al. [CDF Collaboration], J. Phys. G 34, 2457 (2007) [arXiv:hep-ex/0508029].

[70] D. Espriu and J. Manzano, Phys. Rev. D 65, 073005 (2002) [arXiv:hep$\mathrm{ph} / 0107112]$.

[71] T. Sjostrand, L. Lonnblad, S. Mrenna and P. Skands, arXiv:hep$\mathrm{ph} / 0308153$.

[72] F. Maltoni and T. Stelzer, JHEP 0302, 027 (2003) [arXiv:hep-ph/0208156].

[73] M. L. Mangano, M. Moretti, F. Piccinini, R. Pittau and A. D. Polosa, JHEP 0307, 001 (2003) [arXiv:hep-ph/0206293].

[74] R. Brun, R. Hagelberg, M. Hansroul and J. C. Lassalle,

[75] R. Veenhof, Prepared for International Conference on Programming and Mathematical Methods for Solving Physical Problems, Dubna, Russia, 1419 Jun 1993

[76] R. Veenhof, Nucl. Instrum. Meth. A 419, 726 (1998).

[77] G. Grindhammer, M. Rudowicz and S. Peters, Nucl. Instrum. Meth. A 290, 469 (1990).

[78] E. Gerchtein and M. Paulini, In the Proceedings of 2003 Conference for Computing in High-Energy and Nuclear Physics (CHEP 03), La Jolla, California, 24-28 Mar 2003, pp TUMT005 [arXiv:physics/0306031].

[79] S. Richter, "Search for electroweak single top-quark production with the CDF II experiment," CITATION = FERMILAB-THESIS-2007-35;

[80] J. Lueck, "Kinematics of electroweak single top quark production," CITATION = FERMILAB-MASTERS-2006-01;

[81] J. Alwall et al., Eur. Phys. J. C 53, 473 (2008) [arXiv:0706.2569 [hep-ph]].

[82] J. M. Campbell and R. K. Ellis, Phys. Rev. D 60, 113006 (1999) [arXiv:hep$\mathrm{ph} / 9905386]$.

[83] M. Feindt and U. Kerzel, Nucl. Instrum. Meth. A 559, 190 (2006).

[84] C. P. Yuan, Phys. Rev.D 66, 010001 (2002).

[85] A. Abulencia et al. [CDF Collaboration], Phys. Rev. D 73, 112006 (2006) [arXiv:hep-ex/0602008].

[86] A. L. Read, J. Phys. G 28, 2693 (2002).

[87] T. Junk, Nucl. Instrum. Meth. A 434, 435 (1999) [arXiv:hep-ex/9902006]. 
[88] CDF collaboration. Search for Electroweak Single TopQuark Production using Neural Networks with 2.2 $\mathrm{fb}^{-1}$ of CDF II data. Public CDF note 9217, 2008. (http://www-cdf.fnal.gov/physics/new/top/2008/singletop/NN/plots/Conference_Note_NN2fb.pdf)

[89] CDF collaboration. Measurment of Single Top Quark Production in $2.2 \mathrm{fb}^{-1}$ of CDF II Data using the Matrix Element Tchnique. (\{http://www-cdf.fnal.gov/physics/new/top/confNotes/cdf9223_stME_WC2008_v2.pdf)

[90] (http://www-cdf.fnal.gov/physics/new/top/2008/singletop/BDT/SingleTop_BDT.html)

[91] (\{http://www-cdf.fnal.gov/physics/new/top/2008/singletop/s-chan/\})

[92] CDF collaboration. Combination of CDF Single Top Quark Searches with $2.2 \mathrm{fb}^{-1}$ of Data. (http://www-cdf.fnal.gov/physics/new/top/confNotes/cdf9251_stcomb2fbpub.pdf) 


\section{Author's Biography}

Sarah Budd was born Nov. 7, 1980 in Tucson Arizona. She graduated valedictorian of Corvallis High School, Corvallis Oregon, in 1999. In 2003, she graduated from Lewis and Clark College in Portland, Oregon suma cum laude with honors, with a double major in mathematics and physics. At Lewis and Clark, she became a member of Sigma Pi Sigma, Phi Beta Kappa and The American Physical Society (APS). She briefly worked as a girl scout camp counselor before beginning studies in physics in Champaign, Illinois in 2003. She received a Masters in Physics from the University of Illinois in 2004, and joined the Collider Detector at Fermilab (CDF) collaboration shortly thereafter. As part of the CDF collaboration, she is the author of over 111 published and submitted papers. After the completion of her dissertation, Sarah will join the faculty of The University of Redlands in Redlands, California. 